\title{
Trialkylborane-Mediated Propargylation of Aldehydes Using $\gamma$-Stannylated Propargyl Acetates
}

Yoshikazu Horino, ${ }^{* 1}$ Miki Murakami, ${ }^{1}$ Mayo Ishibashi, ${ }^{1}$ Jun Hee Lee ${ }^{2}$, Airi Watanabe, ${ }^{1}$ Rio Matsumoto $^{1}$, and Hitoshi Abe ${ }^{1}$

\author{
${ }^{1}$ Graduate School of Science and Engineering, University of Toyama \\ 3190 Gofuku, Toyama 930-8555, Japan \\ ${ }^{2}$ Department of Advanced Materials Chemistry, Dongguk University \\ Gyeongju 38066, Republic of Korea
}

\section{Table of Contents}

General $\mathrm{S} 2$

Materials .S2

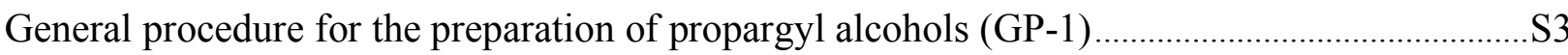

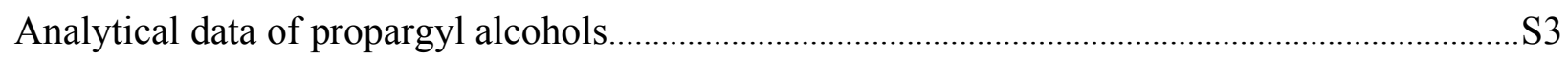

General procedure for the preparation of propargylic acetates (GP-2) ….......................................... 6

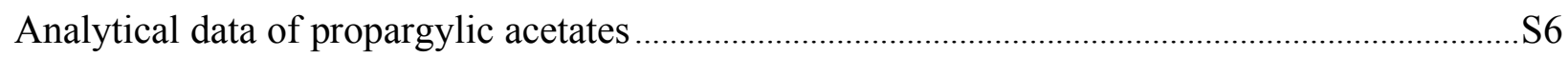

Preparation of 1-phenyl-3-(tributylstannyl)prop-2-yn-1-yl acetate (1a) ........................................S8

General procedure for three-component reaction using 1a, 3a, and

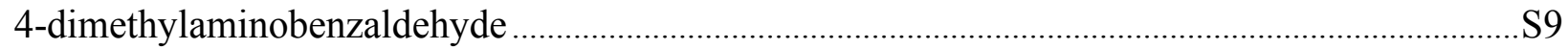

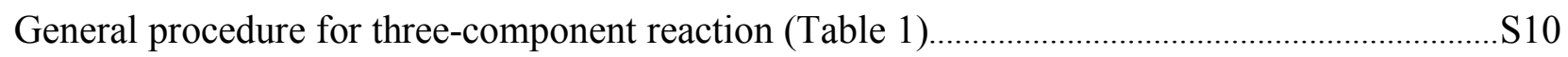

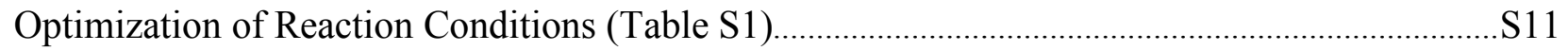

General procedure for three-component reaction using a single flask procedure .............................S11

General procedure for three-component reaction (scope of aldehydes) ................................S12

Analytical data of homopropargylic alcohols ...................................................................................... 12

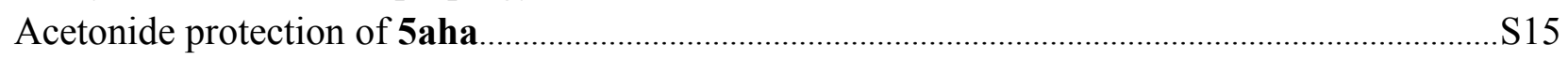

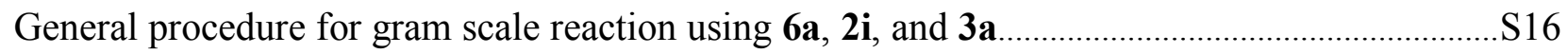

General procedure for three-component reaction (scope of $\gamma$-stannylated propargylic acetates).S18

General procedure for three-component reaction (scope with respected to triorganoboranes)....S24

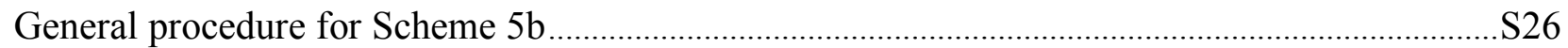

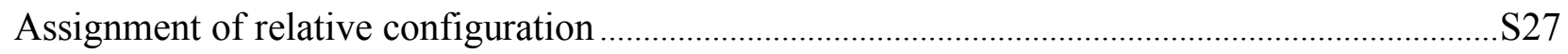

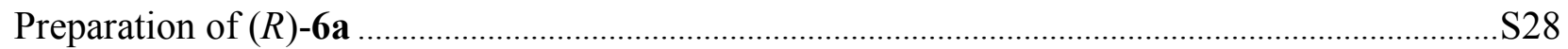

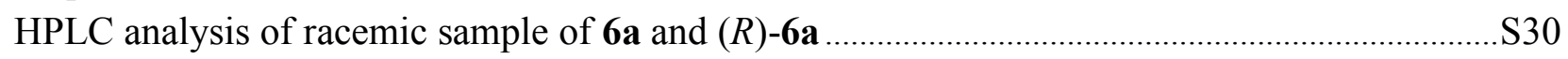

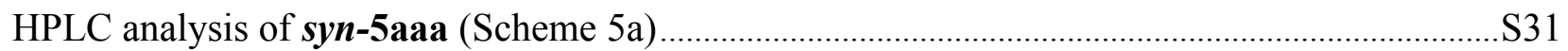

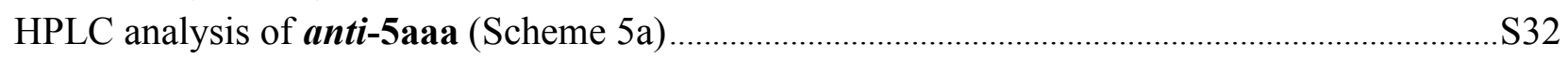

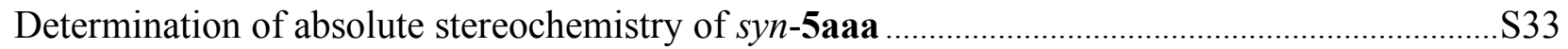

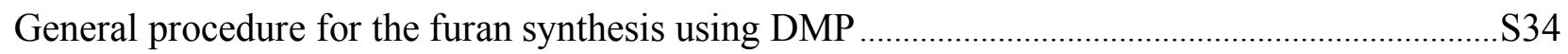

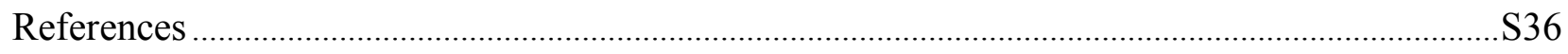

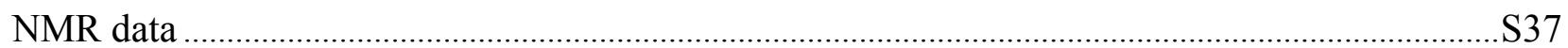




\section{General}

All reactions were carried out in flame-dried glassware under argon atmosphere. Tetrahydrofuran (THF), diethyl ether $\left(\mathrm{Et}_{2} \mathrm{O}\right)$ and toluene were dried over $\mathrm{Na}$ with benzophenone-ketyl intermediate as indicator. Triethylamine, diisopropylamine, and dichloromethane were purified by distillation from $\mathrm{CaH}_{2}$. All commercial reagents were used as received unless otherwise noted.

Flash chromatography was performed with Fuji Silysia PSQ100B $(100 \mu \mathrm{m})$ and KANTO silica gel $60 \mathrm{~N}(63-210 \mu \mathrm{m})$.

Thin layer chromatography (TLC) was carried out using Silicagel 70 F254 TLC Plate-Wako. NMR spectra were recorded on JEOL $\alpha$-GX400 or JNX-ECX500 spectrometer. Chemical shifts $(\delta)$ are reported in ppm and coupling constants are reported in $\mathrm{Hz}$ with $\mathrm{CDCl}_{3}$ referenced at 7.16 $(1 \mathrm{H})$ and $77.16 \mathrm{ppm}(13 \mathrm{C})$, respectively. Peak multiplicities are designated by the following abbreviations: s, singlet; $d$, doublet; t, triplet; q, quartet; m, multiplet; br, broad and coupling constants are provided in (J) Hz. High-resolution mass (HRMS) spectral data were obtained on a JEOL MStation JMS-700 (EI was reduced to 30-50 eV instead of the normal $70 \mathrm{eV}$ ). The collision energy $20 \mathrm{eV}$ was used for LRMS. Chiral HPLC was performed on a Waters HPLC (pump: Waters 600 pump). The specific rotation of a chiral compound was determined on a HORIBA SEPA-500 apparatus and transformed for a given temperature according to the following formula.

$$
[\alpha]_{D}^{T}=\frac{\alpha \cdot 100}{c \cdot d}
$$

$\alpha$ : measured value for optical rotation; $c$ : concentration in $\mathrm{g} / 100 \mathrm{~mL} ; d$ : length of the quartz cuvette in $\mathrm{dm}$; $T$ : temperature in ${ }^{\circ} \mathrm{C}$.

\section{Materials}

Tributyltin chloride (Acros), 4-dimethylaminobenzaldehyde (nakarai), 2-methoxybenzaldehyde (TCI), 3-methoxybenzaldehyde (TCI), 4-methoxybenzaldehyde (TCI), methyl 4-formylbenzoate (TCI), 4-bromobenzaldehyde (TCI), 2-bromobenzaldehyde (TCI), 2-furaldehyde (TCI), 2thiophenecarboxaldehyde (TCI), cyclohexanecarboxaldehyde (TCI), cyclopentanone (TCI), benzaldehyde (TCI), 3-iodobenzaldehyde (TCI), 4-hydroxybenzaldehyde (TCI), 2hydroxybenzaldehyde (TCI), 2,4-dimethoxybenzaldehyde (TCI), methyl 2-formylbenzoate (TCI), trans-cinnamaldehyde (TCI), isobutyraldehyde (TCI), cyclopropanecarboxaldehyde (Aldrich), ethynylmagnesium chloride (0.5 M in THF, Aldrich), $n$-BuLi (WAKO), triethylamine (KANTO), 1-phenylethylamine (TCI), diisopropylamine (TCI), 2,2,6,6-tetramethylpiperidine (TCI), acetic anhydride (TCI), triethylborane ( $1 \mathrm{M}$ in hexane, KANTO), tri- $n$-butylborane ( $1 \mathrm{M}$ in diethyl ether, Aldrich), 4-(dimethylamino)pyridine (TCI), borane dimethyl sulfide complex (TCI), Lindlar catalyst (TCI) were purchased. 


\section{General Procedure for the preparation of propargyl alcohols (GP-1)}

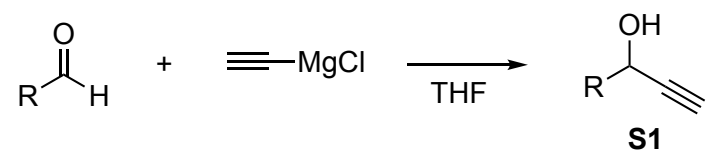

A typical procedure for the preparation of propargyl alcohols is described for the reaction using benzaldehyde:

To a solution of benzaldehyde $(1.06 \mathrm{~g}, 10 \mathrm{mmol})$ in THF $(20 \mathrm{~mL})$ was added slowly ethynylmagnesium chloride $(0.5 \mathrm{M}$ in THF, $24 \mathrm{~mL}, 12 \mathrm{mmol})$ at $0{ }^{\circ} \mathrm{C}$ under argon atmosphere. The reaction mixture was allowed to warm up to room temperature, and the progress of the reaction was monitored by TLC. Upon completion, the reaction mixture was diluted with ethyl acetate $(50 \mathrm{~mL})$, washed with water $(50 \mathrm{~mL})$, saturated aqueous $\mathrm{NH}_{4} \mathrm{Cl}(2 \times 30 \mathrm{~mL})$, and then brine $(2 \times 30 \mathrm{~mL})$. The organic layer was dried over $\mathrm{MgSO}_{4}$, filtered, and concentrated in vacuo. The residue was purified by silica gel column chromatography to give 1-phenyl-2-propyn-1-ol (S1a) as a yellow oil (1.64 g, 94\%, $\mathrm{R}_{f} 0.51$, EtOAc/hexane $\left.=2 / 8\right)$.

The spectral data of 1-phenyl-2-propyn-1-ol (S1a) are consistent with those of the literature. ${ }^{[1]}$

${ }^{1} \mathbf{H}$ NMR $\left(400 \mathrm{MHz}, \mathrm{CDCl}_{3}\right) \delta 7.60-7.52(\mathrm{~m}, 2 \mathrm{H}), 7.42-7.31(\mathrm{~m}, 3 \mathrm{H}), 5.47(\mathrm{~d}, J=2.4 \mathrm{~Hz}, 1 \mathrm{H})$, $2.68(\mathrm{~d}, J=2.4 \mathrm{~Hz}, 1 \mathrm{H}), 2.06(\mathrm{br} \mathrm{s}, 1 \mathrm{H})$.

Compounds S1a (CAS:4187-87-5) ${ }^{[1]}$, S1b (CAS:1776-12-1 $)^{[2], ~ S 1 c ~(C A S: ~ 222713-56-6) ~}{ }^{[3]}$, S1d (CAS:19115-30-1) ${ }^{[1]}$, S1e (CAS:178110-08-2) ${ }^{[4]}$, S1f (CAS: 85020-75-3) ${ }^{[5]}$, S1g (CAS: 17924884-1) $)^{[1]}$, S1h (CAS: 695-82-9) ${ }^{[5]}$, S1i (CAS: 19212-35-2) ${ }^{[6]}$, S1j (CAS: 4187-88-6) ${ }^{[7]}$, and S1k (CAS:17356-19-3 $)^{[8]}$ are known.

Compounds S1d (CAS:19115-30-1) ${ }^{[1]}$, S1f (CAS: 85020-75-3) ${ }^{[5]}$, S1g (CAS: 179248-84-1) ${ }^{[1]}$, S1h (CAS: 695-82-9) ${ }^{[5]}$, and S1k (CAS:17356-19-3) ${ }^{[6]}$ were used for next reaction without further purification.<smiles>C#CC(O)c1ccccc1OC</smiles>

\section{1-(2-Methoxyphenyl)-2-propyn-1-ol (S1b, CAS:1776-12-1) $)^{[2]}$}

Following the general procedure (GP-1); reaction of 2-methoxybenzaldehyde (3.00 g, $22 \mathrm{mmol})$ and ethynylmagnesium chloride $(0.5 \mathrm{M}$ in THF, $53 \mathrm{~mL}, 26.5 \mathrm{mmol})$ gave $\mathbf{S 1 b}\left(3.50 \mathrm{~g}, 99 \%, \mathrm{R}_{f}\right.$ $0.30 \mathrm{EtOAc} /$ hexane $=3 / 7)$. The spectral data of $\mathbf{S 1 b}$ are consistent with those of the literature. ${ }^{[2]}$

${ }^{1} \mathbf{H}$ NMR $\left(400 \mathrm{MHz}, \mathrm{CDCl}_{3}\right) \delta 7.56(\mathrm{dd}, J=2.0,8.0 \mathrm{~Hz}, 1 \mathrm{H}), 7.32(\mathrm{dt}, J=1.6,8.0 \mathrm{~Hz}, 1 \mathrm{H}), 6.99$ $(\mathrm{t}, J=8.0 \mathrm{~Hz}, 1 \mathrm{H}), 6.91(\mathrm{~d}, J=8.0 \mathrm{~Hz}, 1 \mathrm{H}), 5.69(\mathrm{dd}, J=2.4,6.0 \mathrm{~Hz}, 1 \mathrm{H}), 3.89(\mathrm{~s}, 3 \mathrm{H}), 3.11(\mathrm{br} \mathrm{s}$, $1 \mathrm{H}), 2.61(\mathrm{~d}, J=2.4 \mathrm{~Hz}, 1 \mathrm{H})$.<smiles>C#CC(O)c1ccccc1Br</smiles>

1-(2-Bromophenyl)-2-propyn-1-ol (S1c, CAS: 222713-56-6) ${ }^{[3]}$

Following the general procedure (GP-1); reaction of 2-bromobenzaldehyde $(1.85 \mathrm{~g}, 10 \mathrm{mmol})$ and ethynylmagnesium chloride $(0.5 \mathrm{M}$ in THF, $24 \mathrm{~mL}, 12 \mathrm{mmol})$ gave $\mathbf{S 1 c}\left(1.84 \mathrm{~g}, 87 \%, \mathrm{R}_{f} 0.41\right.$, EtOAc/hexane $=3 / 7)$. The spectral data of S1c are consistent with those of the literature. ${ }^{[3]}$ 
${ }^{1} \mathbf{H}$ NMR $\left(400 \mathrm{MHz}, \mathrm{CDCl}_{3}\right) \delta 7.79(\mathrm{dd}, J=1.6,7.6 \mathrm{~Hz}, 1 \mathrm{H}), 7.57(\mathrm{dd}, J=1.2,7.6 \mathrm{~Hz}, 1 \mathrm{H}), 7.38$ $(\mathrm{dt}, J=1.6,8.0 \mathrm{~Hz}, 1 \mathrm{H}), 7.21(\mathrm{dt}, J=2.4,7.6 \mathrm{~Hz}, 1 \mathrm{H}), 5.80(\mathrm{dd}, J=2.4,5.2 \mathrm{~Hz}, 1 \mathrm{H}), 2.67(\mathrm{~d}, \mathrm{~J}=$ $2.4 \mathrm{~Hz}, 1 \mathrm{H}), 2.51(\mathrm{~d}, J=5.2 \mathrm{~Hz}, 1 \mathrm{H})$.<smiles>C#CC(O)c1ccc(OC)cc1</smiles>

\section{1-(4-Methoxyphenyl)-2-propyn-1-ol (S1d, CAS:19115-30-1) ${ }^{[1]}$}

Following the general procedure (GP-1); reaction of 4-methoxybenzaldehyde (1.36 g, $10 \mathrm{mmol})$ and ethynylmagnesium chloride $(0.5 \mathrm{M}$ in THF, $24 \mathrm{~mL}, 12 \mathrm{mmol})$ gave S1d $\left(\mathrm{R}_{f} 0.29\right.$, EtOAc/hexane $=3 / 7$ ). S1d was used for next reaction without further purification. The spectral data of S1c are consistent with those of the literature. ${ }^{[1]}$

${ }^{1} \mathbf{H}$ NMR $\left(400 \mathrm{MHz}, \mathrm{CDCl}_{3}\right) \delta 7.48(\mathrm{dm}, J=8.4 \mathrm{~Hz}, 2 \mathrm{H}), 6.91(\mathrm{dm}, J=8.4 \mathrm{~Hz}, 2 \mathrm{H}), 5.42(\mathrm{br} \mathrm{s}$, $1 \mathrm{H}), 3.82(\mathrm{~s}, 3 \mathrm{H}), 2.66(\mathrm{~d}, J=2.4 \mathrm{~Hz}, 1 \mathrm{H}), 2.18$ (br s, 1H).<smiles>C#CC(O)c1ccc(C(C)=O)cc1</smiles>

\section{1-[4-(Methoxycarbonyl)phenyl]-2-propyn-1-ol (S1e, CAS:178110-08-2) ${ }^{[4]}$}

Following the general procedure (GP-1); reaction of methyl 4-formylbenzoate $(2.38 \mathrm{~g}, 14.5 \mathrm{mmol})$ and ethynylmagnesium chloride $(0.5 \mathrm{M}$ in THF, $35 \mathrm{~mL}, 17.5 \mathrm{mmol})$ gave S1e $\left(2.46 \mathrm{~g}, 89 \% \mathrm{R}_{f}\right.$ 0.38$, EtOAc/hexane $=3 / 7)$. The spectral data of S1c are consistent with those of the literature. ${ }^{[4]}$

${ }^{1} \mathbf{H}$ NMR $\left(400 \mathrm{MHz}, \mathrm{CDCl}_{3}\right) \delta 7.99(\mathrm{dd}, J=2.4,8.4 \mathrm{~Hz}, 2 \mathrm{H}), 7.56(\mathrm{dm}, J=8.4 \mathrm{~Hz}, 2 \mathrm{H}), 5.46(\mathrm{~d}, J$ $=2.4,6.0 \mathrm{~Hz}, 1 \mathrm{H}), 3.89(\mathrm{~s}, 3 \mathrm{H}), 2.63(\mathrm{~d}, J=2.4 \mathrm{~Hz}, 1 \mathrm{H}), 2.30(\mathrm{~d}, J=6.0 \mathrm{~Hz}, 1 \mathrm{H})$.<smiles>C#CC(O)c1ccc(Br)cc1</smiles>

\section{1-(4-Bromophenyl)-2-propyn-1-ol (S1f, CAS: 85020-75-3) ${ }^{[5]}$}

Following the general procedure (GP-1); reaction of 4-bromobenzaldehyde $(1.85 \mathrm{~g}, 10 \mathrm{mmol})$ and ethynylmagnesium chloride $(0.5 \mathrm{M}$ in THF, $24 \mathrm{~mL}, 12 \mathrm{mmol})$ gave $\mathbf{S 1 f}\left(\mathrm{R}_{f} 0.38\right.$, EtOAc/hexane $=$ 3/7). S1d was used for next reaction without further purification. The spectral data of S1f are consistent with those of the literature. ${ }^{[5]}$

${ }^{1}$ H NMR $\left(400 \mathrm{MHz} \mathrm{CDCl}_{3}\right) \delta 7.52(\mathrm{dm}, J=8.4 \mathrm{~Hz}, 2 \mathrm{H}), 7.43(\mathrm{dm}, J=8.4 \mathrm{~Hz}, 2 \mathrm{H}), 5.43(\mathrm{~d}, J=$ $2.4 \mathrm{~Hz}, 1 \mathrm{H}), 2.68$ (d, $J=2.4 \mathrm{~Hz}, 1 \mathrm{H}), 2.30$ (br s, $1 \mathrm{H})$.<smiles>C#CC(O)c1cccc(OC)c1</smiles>

\section{1-(3-Methoxyphenyl)-2-propyn-1-ol (S1g, CAS: 179248-84-1) ${ }^{[1]}$}

Following the general procedure (GP-1); reaction of 4-methoxybenzaldehyde (1.36 g, $10 \mathrm{mmol})$ and ethynylmagnesium chloride $(0.5 \mathrm{M}$ in THF, $24 \mathrm{~mL}, 12 \mathrm{mmol})$ gave $\mathbf{S 1 d}\left(\mathrm{R}_{f} 0.30\right.$, EtOAc/hexane = 3/7). S1g was used for next reaction without further purification. The spectral data of S1g are consistent with those of the literature. ${ }^{[1]}$

${ }^{1} \mathbf{H}$ NMR $\left(400 \mathrm{MHz}, \mathrm{CDCl}_{3}\right) \delta 7.30(\mathrm{t}, J=8.0,1 \mathrm{H}), 7.15-7.09(\mathrm{~m}, 2 \mathrm{H}), 6.88(\mathrm{dm}, J=8.0,1 \mathrm{H})$, 5.44 (br s, 1H), 3.82 (s, 3H), 2.66 (d, $J=2.4 \mathrm{~Hz}, 1 \mathrm{H}), 2.39$ (br s, 1H). 
<smiles>C#CC(O)c1ccco1</smiles>

\section{1-(Furan-2-yl)prop-2-yn-1-ol (S1h, CAS: 695-82-9) ${ }^{[5]}$}

Following the general procedure (GP-1); reaction of 2 -furaldehyde $(480.5 \mathrm{mg}, 5 \mathrm{mmol})$ and ethynylmagnesium chloride $(0.5 \mathrm{M}$ in THF, $12 \mathrm{~mL}, 6 \mathrm{mmol})$ gave $\mathbf{S 1 h} .\left(\mathrm{R}_{f} 0.27\right.$, EtOAc/hexane $=$ 3/7). S1h was used for next reaction without further purification. The spectral data of $\mathbf{S 1 h}$ are consistent with those of the literature. ${ }^{[5]}$

${ }^{1} \mathbf{H}$ NMR $\left(400 \mathrm{MHz}, \mathrm{CDCl}_{3}\right) \delta 7.42(\mathrm{~d}, J=2.0 \mathrm{~Hz}, 1 \mathrm{H}), 6.47(\mathrm{dm}, J=2.8 \mathrm{~Hz}, 1 \mathrm{H}), 6.36(\mathrm{dd}, \mathrm{J}=$ $2.0,3.6 \mathrm{~Hz}, 1 \mathrm{H}), 5.46(\mathrm{dd}, J=2.4,6.0 \mathrm{~Hz}, 1 \mathrm{H}), 2.62(\mathrm{~d}, J=2.4 \mathrm{~Hz}, 1 \mathrm{H}), 2.41(\mathrm{~d}, J=6.0 \mathrm{~Hz}, 1 \mathrm{H})$.<smiles>C#CC(O)c1cccs1</smiles>

\section{1-(Thiophen-2-yl)prop-2-yn-1-ol (S1i, CAS: 19212-35-2) ${ }^{[6]}$}

Following the general procedure (GP-1); reaction of 2-thiophenecarboxaldehyde $(560.8 \mathrm{mg}, 5$ mmol) and ethynylmagnesium chloride $(0.5 \mathrm{M}$ in THF, $12 \mathrm{~mL}, 6 \mathrm{mmol})$ gave S1i $(545.9 \mathrm{mg}$, $79 \%, \mathrm{R}_{f} 0.34$, EtOAc/hexane $\left.=3 / 7\right)$. The spectral data of S1c are consistent with those of the literature. ${ }^{[6]}$

${ }^{1} \mathbf{H}$ NMR $\left(400 \mathrm{MHz}, \mathrm{CDCl}_{3}\right) \delta 7.32(\mathrm{dd}, J=1.6,5.2 \mathrm{~Hz}, 1 \mathrm{H}), 7.20(\mathrm{dm}, J=4.0 \mathrm{~Hz}, 1 \mathrm{H}), 6.99(\mathrm{dd}$, $J=4.0,5.2 \mathrm{~Hz}, 1 \mathrm{H}), 5.66(\mathrm{dd}, J=2.4,6.4 \mathrm{~Hz}, 1 \mathrm{H}), 2.69(\mathrm{~d}, J=2.4 \mathrm{~Hz}, 1 \mathrm{H}), 2.44(\mathrm{~d}, J=6.4 \mathrm{~Hz}$, $1 \mathrm{H})$.<smiles>C#CC(O)C1CCCCC1</smiles>

\section{1-Cyclohexyl-2-propyn-1-ol (S1j, CAS: 4187-88-6) ${ }^{[7]}$}

Following the general procedure (GP-1); reaction of 2-thiophenecarboxaldehyde $(560.8 \mathrm{mg}, 5$ $\mathrm{mmol}$ ) and ethynylmagnesium chloride (0.5 M in THF, $12 \mathrm{~mL}, 6 \mathrm{mmol})$ gave $\mathbf{S 1 j}$ (481.6 mg, 70\%, $\mathrm{R}_{f} 0.26$, EtOAc/hexane $\left.=1 / 4\right)$. The spectral data of S1c are consistent with those of the literature. ${ }^{[7]}$

${ }^{1} \mathbf{H}$ NMR $\left(400 \mathrm{MHz}, \mathrm{CDCl}_{3}\right) \delta 4.16(\mathrm{dt}, J=2.4,6.0 \mathrm{~Hz}, 1 \mathrm{H}), 2.46(\mathrm{~d}, J=2.4 \mathrm{~Hz}, 1 \mathrm{H}), 1.89-1.82$ $(\mathrm{m}, 2 \mathrm{H}), 1.82-1.75(\mathrm{~m}, 3 \mathrm{H}), 1.69(\mathrm{~m}, 1 \mathrm{H}), 1.57(\mathrm{~m}, 1 \mathrm{H}), 1.32-1.02(\mathrm{~m}, 5 \mathrm{H})$.<smiles>C#CC1(O)CCCC1</smiles>

\section{1-Ethynylcyclopentanol (S1k, CAS:17356-19-3) ${ }^{[8]}$}

Following the general procedure (GP-1); reaction of cyclopentanone $(561.1 \mathrm{mg}, 6.7 \mathrm{mmol})$ and ethynylmagnesium chloride $(0.5 \mathrm{M}$ in THF, $16 \mathrm{~mL}, 8 \mathrm{mmol})$ gave $\mathbf{S 1} \mathbf{k}\left(\mathrm{R}_{f} 0.45\right.$, EtOAc/hexane $=$ 3/7). S1k was used for next reaction without further purification. The spectral data of S1k are consistent with those of the literature. ${ }^{[8]}$

${ }^{1}$ H NMR $\left(400 \mathrm{MHz}, \mathrm{CDCl}_{3}\right) \delta 2.49(\mathrm{~s}, 1 \mathrm{H}), 1.05-70(\mathrm{~m}, 9 \mathrm{H})$. 


\section{General Procedure for the preparation of propargylic acetates (GP-2)}

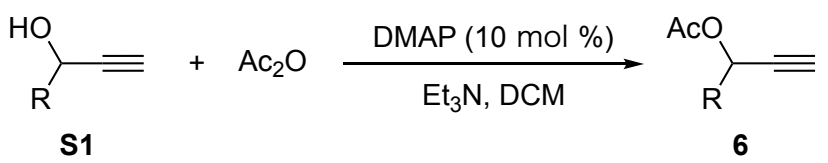

A typical procedure for the preparation of propargyl alcohols is described for the reaction using 1phenyl-2-propyn-1-ol (S1a) as a substrate: To a solution of 1-phenyl-2-propyn-1-ol (S1a) (1.60 g, $7.70 \mathrm{mmol})$, N,N-dimethyl-4-aminopyridine $(95.5 \mathrm{mg}, 0.77 \mathrm{mmol})$ and $\mathrm{Et}_{3} \mathrm{~N}(2.1 \mathrm{~mL}, 15.4 \mathrm{mmol})$ in DCM $(20 \mathrm{~mL})$ was added acetic acid anhydride $(1.1 \mathrm{~mL}, 11.6 \mathrm{mmol})$ at $0{ }^{\circ} \mathrm{C}$ under argon atmosphere. After stirring at room temperature for 1 hour, the reaction mixture was quenched with saturated aqueous $\mathrm{NH}_{4} \mathrm{Cl}$. The aqueous phase was extracted with $\mathrm{DCM}(2 \times 30 \mathrm{~mL})$, and the combined organic extracts were washed with brine $(2 \times 20 \mathrm{~mL})$. After the organic layer was dried over $\mathrm{MgSO}_{4}$, the solvent was removed under reduced pressure. The residue was purified by silica gel column chromatography $(\mathrm{EtOAc} /$ hexane $=1 / 9)$ to give 1 -phenylpropargyl acetate $(\mathbf{6 a})$ as a colorless oil (1.6 g, 85\% R 0.52 , EtOAc/hexane $=3 / 7)$.

The spectral data of 1-phenylprop-2-yn-1-yl acetate (6a) are consistent with those of the literature. $^{[4]}$

${ }^{1} \mathbf{H}$ NMR $\left(400 \mathrm{MHz}, \mathrm{CDCl}_{3}\right) \delta 7.56-7.51(\mathrm{~m}, 2 \mathrm{H}), 7.42-7.34(\mathrm{~m}, 3 \mathrm{H}), 6.45(\mathrm{~d}, J=2.4 \mathrm{~Hz}, 1 \mathrm{H})$, $2.65(\mathrm{~d}, J=2.4 \mathrm{~Hz}, 1 \mathrm{H}), 2.11(\mathrm{~s}, 3 \mathrm{H})$.

Compounds 6a (CAS:16169-88-3 $)^{[4]}$, 6b (CAS: 179248-89-6) ${ }^{[9]}$, 6c (CAS: 1007562-20-0) ${ }^{[9]}$, 6d (CAS: 99520-55-5) ${ }^{[10]}$, 6f (CAS: 960377-64-4) ${ }^{[10]}$, 6g (CAS: 179249-08-2) ${ }^{[9]}$, , 6h (CAS: 1927525-3) $)^{[5]}$, 6i (CAS: 38597-85-2) ${ }^{[10]}$, 6j (CAS: 16169-86-1) $)^{[13]}$, and 6k (CAS: 36193-52-9) $)^{[12]}$ are known.<smiles>C#CC(OC)c1ccccc1OC</smiles>

\section{1-(2-Methoxyphenyl)prop-2-yn-1-yl acetate (6b, CAS: 179248-89-6) ${ }^{[9]}$}

Following the general procedure (GP-2); reaction of S1b $(1.62 \mathrm{~g}, 10 \mathrm{mmol}), \mathrm{N}, \mathrm{N}$-dimethyl-4aminopyridine $(122.2 \mathrm{mg}, 1 \mathrm{mmol}), \mathrm{Et}_{3} \mathrm{~N}(2.8 \mathrm{~mL}, 20 \mathrm{mmol})$, and acetic anhydride $(1.4 \mathrm{~mL}, 15$ $\mathrm{mmol}$ ) gave $\mathbf{6 b}$ (colorless oil, $1.98 \mathrm{~g}, 70 \%, \mathrm{R}_{f} 0.42$, EtOAc/hexane $\left.=1 / 4\right)$. The spectral data of $\mathbf{6 c}$ are consistent with those of the literature. ${ }^{[9]}$

${ }^{1} \mathbf{H}$ NMR $\left(400 \mathrm{MHz}, \mathrm{CDCl}_{3}\right) \delta 7.67(\mathrm{dd}, J=2.0 .8 .0 \mathrm{~Hz}, 1 \mathrm{H}), 7.35(\mathrm{dt}, J=2.0,8.0 \mathrm{~Hz}, 1 \mathrm{H}), 7.01$ $(\mathrm{dt}, J=1.2,8.0 \mathrm{~Hz}, 1 \mathrm{H}), 6.90(\mathrm{~d}, J=8.0 \mathrm{~Hz}, 1 \mathrm{H}), 6.79(\mathrm{~d}, J=2.4 \mathrm{~Hz}, 1 \mathrm{H}), 3.84(\mathrm{~s}, 3 \mathrm{H}), 2.59(\mathrm{~d}, J$ $=2.4 \mathrm{~Hz}, 1 \mathrm{H}), 2.10(\mathrm{~s}, 3 \mathrm{H})$.<smiles>C#CC(OC(C)=O)c1ccccc1Br</smiles>

1-(2-Bromophenyl)prop-2-yn-1-yl acetate (6c, CAS: 1007562-20-0) ${ }^{[9]}$

Following the general procedure (GP-2); reaction of S1c $(1.77 \mathrm{~g}, 8.4 \mathrm{mmol})$, N,N-dimethyl-4aminopyridine $(102.6 \mathrm{mg}, 0.84 \mathrm{mmol}), \mathrm{Et}_{3} \mathrm{~N}(2.3 \mathrm{~mL}, 16.8 \mathrm{mmol})$, and acetic anhydride $(1.2 \mathrm{~mL}$, $12.6 \mathrm{mmol}$ ) gave $\mathbf{6 c}$ (pale yellow oil, $2.0 \mathrm{~g}, 95 \%, \mathrm{R}_{f} 0.53$, EtOAc/hexane $=3 / 7$ ). The spectral data of $\mathbf{6 c}$ are consistent with those of the literature. ${ }^{[9]}$

6c $\left(8.4 \mathrm{mmol}\right.$ scale reaction, $2.0 \mathrm{~g}, 95 \%, \mathrm{R}_{f} 0.53$, EtOAc/hexane $\left.=3 / 7\right)$, 
${ }^{1} \mathrm{H}$ NMR $\left(400 \mathrm{MHz}, \mathrm{CDCl}_{3}\right) \delta 7.79(\mathrm{dd}, J=2.0,8.0 \mathrm{~Hz}, 1 \mathrm{H}), 7.59(\mathrm{~d}, J=1.2,8.0 \mathrm{~Hz}, 1 \mathrm{H}), 7.39$ $(\mathrm{dt}, J=1.2,8.0 \mathrm{~Hz}, 1 \mathrm{H}), 7.25(\mathrm{dt}, J=1.2,8.0 \mathrm{~Hz}, 1 \mathrm{H}), 6.68(\mathrm{~d}, J=2.0 \mathrm{~Hz}, 1 \mathrm{H}), 2.68(\mathrm{~d}, J=2.0$ $\mathrm{Hz}, 1 \mathrm{H}), 2.11(\mathrm{~s}, 3 \mathrm{H})$.<smiles>C#CC(OC(C)=O)c1ccc(OC)cc1</smiles>

1-(4-Methoxyphenyl)prop-2-yn-1-yl acetate (6d, CAS: 99520-55-5) ${ }^{[10]}$

Following the general procedure (GP-2); reaction of crude S1d (10 mmol), N,N-dimethyl-4aminopyridine $(122.2 \mathrm{mg}, 1 \mathrm{mmol}), \mathrm{Et}_{3} \mathrm{~N}(2.8 \mathrm{~mL}, 20 \mathrm{mmol})$, and acetic anhydride $(1.4 \mathrm{~mL}, 15$ mmol) gave 6d (colorless oil, $1.90 \mathrm{~g}, 93 \%$ for 2 steps, $\mathrm{R}_{f} 0.47$, EtOAc/hexane $=3 / 7$ ).

${ }^{1} \mathbf{H}$ NMR $\left(400 \mathrm{MHz}, \mathrm{CDCl}_{3}\right) \delta 7.47(\mathrm{dm}, J=8.4 \mathrm{~Hz}, 2 \mathrm{H}), 6.91(\mathrm{dm} J=8.4 \mathrm{~Hz}, 2 \mathrm{H}), 6.41(\mathrm{~d}, J=$ $2.4 \mathrm{~Hz}, 1 \mathrm{H}), 3.82$ (s, 3), 2.65 (d, $J=2.4 \mathrm{~Hz}, 1 \mathrm{H}), 2.09$ (s, 3H).<smiles>C#CC(OC(C)=O)c1ccc(C(C)=O)cc1</smiles>

\section{4-[1-(Acetyloxy)-2-propyn-1-yl]benzoic acid methyl ester (6e, CAS: 2119729-24-5)}

Following the general procedure (GP-2); reaction of S1e (1.90 g, $10 \mathrm{mmol})$, N,N-dimethyl-4aminopyridine (122.2 $\mathrm{mg}, 1 \mathrm{mmol}), \mathrm{Et}_{3} \mathrm{~N}(2.8 \mathrm{~mL}, 20 \mathrm{mmol})$, and acetic anhydride $(1.4 \mathrm{~mL}, 15$ $\mathrm{mmol}$ ) gave 6e (colorless oil, $1.76 \mathrm{~g}, 76 \%, \mathrm{R}_{f} 0.43$, EtOAc/hexane $=3 / 7$ ).

${ }^{1}$ H NMR $(400 \mathrm{MHz}, \mathrm{CDCl} 3) \delta 8.06(\mathrm{dm}, J=8.0 \mathrm{~Hz}, 2 \mathrm{H}), 7.60(\mathrm{dm}, J=8.0 \mathrm{~Hz}, 2 \mathrm{H}), 6.48(\mathrm{~d}, J=$ $2.4 \mathrm{~Hz}, 1 \mathrm{H}), 3.93(\mathrm{~s}, 3 \mathrm{H}), 2.69(\mathrm{~d}, J=2.4 \mathrm{~Hz}, 1 \mathrm{H}), 2.14(\mathrm{~s}, 3 \mathrm{H}) ;{ }^{13} \mathbf{C}$ NMR $\left(100 \mathrm{MHz}, \mathrm{CDCl}_{3}\right) \delta$ 169.6, 166.6, 141.2, 130.8, 130.1, 127.6, 79.7, 76.1, 64.8, 52.4, 21.0; HRMS $m / z\left(\mathrm{M}^{+}\right)$calcd for $\mathrm{C}_{13} \mathrm{H}_{12} \mathrm{O}_{4}: 232.0736\left(\mathrm{M}^{+}\right)$, found: 232.0737.<smiles>C#CC(OC(C)=O)c1ccc(Br)cc1</smiles>

1-(4-Bromophenyl)prop-2-yn-1-yl acetate (6f, CAS: 960377-64-4) ${ }^{[10]}$

Following the general procedure (GP-2); reaction of crude S1f $(10 \mathrm{mmol})$, N,N-dimethyl-4aminopyridine (122.2 mg, $1 \mathrm{mmol}), \mathrm{Et}_{3} \mathrm{~N}(2.8 \mathrm{~mL}, 20 \mathrm{mmol})$, and acetic anhydride $(1.4 \mathrm{~mL}, 15$ mmol) gave $6 \mathbf{f}$ (pale yellow oil, $2.26 \mathrm{~g}, 90 \%$ for 2 steps, $\mathrm{R}_{f} 0.53$, EtOAc/hexane $=3 / 7$ ).

${ }^{1} \mathbf{H}$ NMR $\left(400 \mathrm{MHz}, \mathrm{CDCl}_{3}\right) \delta 7.52(\mathrm{dm}, J=8.4 \mathrm{~Hz}, 2 \mathrm{H}), 7.41(\mathrm{dm}, J=8.4 \mathrm{~Hz}, 2 \mathrm{H}), 6.40(\mathrm{~d}, J=$ $2.4 \mathrm{~Hz}, 1 \mathrm{H}), 2.67(\mathrm{~d}, J=2.4 \mathrm{~Hz}, 1), 2.11(\mathrm{~s}, 3 \mathrm{H})$.<smiles>C#CC(OC(C)=O)c1cccc(OC)c1</smiles>

\section{1-(3-Methoxyphenyl)prop-2-yn-1-yl acetate (6g, CAS: 179249-08-2) ${ }^{[9]}$}

Following the general procedure (GP-2); reaction of crude S1g (10 mmol), N,N-dimethyl-4aminopyridine $(122.2 \mathrm{mg}, 1 \mathrm{mmol}), \mathrm{Et}_{3} \mathrm{~N}(2.8 \mathrm{~mL}, 20 \mathrm{mmol})$, and acetic anhydride $(1.4 \mathrm{~mL}, 15$ mmol) gave 6g (colorless oil, $1.83 \mathrm{~g}, 90 \%$ for 2 steps, $\mathrm{R}_{f} 0.43$, EtOAc/hexane $=3 / 7$ ).

${ }^{1} \mathbf{H}$ NMR $\left(400 \mathrm{MHz}, \mathrm{CDCl}_{3}\right) \delta 7.30(\mathrm{t}, J=8.0 \mathrm{~Hz}, 1 \mathrm{H}), 7.10(\mathrm{~d}, J=8.0 \mathrm{~Hz}, 1 \mathrm{H}), 7.08(\mathrm{t}, J=2.4$ $\mathrm{Hz}, 1 \mathrm{H}), 6.91(\mathrm{dd}, J=2.4,8.0 \mathrm{~Hz}, 1 \mathrm{H}), 6.42(\mathrm{~d}, J=2.4 \mathrm{~Hz}, 1 \mathrm{H}), 3.83(\mathrm{~s}, 3 \mathrm{H}), 2.64(\mathrm{~d}, J=2.4 \mathrm{~Hz}$, $1 \mathrm{H}), 2.12(\mathrm{~s}, 3 \mathrm{H})$. 
<smiles>C#CC(OC(C)=O)c1ccco1</smiles>

\section{1-(Furan-2-yl)prop-2-yn-1-yl acetate (6h, CAS: 19275-25-3) ${ }^{[5]}$}

Following the general procedure (GP-2); reaction of crude S1h (5 mmol), N,N-dimethyl-4aminopyridine (yellow oil, $102.6 \mathrm{mg}, 0.84 \mathrm{mmol}$ ), $\mathrm{Et}_{3} \mathrm{~N}(2.3 \mathrm{~mL}, 16.8 \mathrm{mmol}$ ), and acetic anhydride $(1.2 \mathrm{~mL}, 12.6 \mathrm{mmol})$ gave $\mathbf{6 h}\left(614.0 \mathrm{mg}, 75 \%\right.$ for 2 steps, $\mathrm{R}_{f} 048$, EtOAc/hexane $\left.=3 / 7\right)$. ${ }^{1} \mathbf{H}$ NMR $\left(400 \mathrm{MHz}, \mathrm{CDCl}_{3}\right) \delta 7.44($ br s, $1 \mathrm{H}), 6.57(\mathrm{~d}, J=3.2 \mathrm{~Hz}, 1 \mathrm{H}), 6.50(\mathrm{~d}, J=2.4 \mathrm{~Hz}, 1 \mathrm{H})$, $6.39(\mathrm{dd}, J=2.0,3.2 \mathrm{~Hz}, 1 \mathrm{H}), 2.62(\mathrm{~d}, J=2.4 \mathrm{~Hz}, 1 \mathrm{H}), 2.12(\mathrm{~s}, 3 \mathrm{H})$.<smiles>C#CC(OC(C)=O)c1cccs1</smiles>

\section{1-(Thiophen-2-yl)prop-2-yn-1-yl acetate (6i, CAS: 38597-85-2) ${ }^{[10]}$}

Following the general procedure (GP-2); reaction of S1i (497.5 mg, $3.6 \mathrm{mmol})$, N,N-dimethyl-4aminopyridine $(43.9 \mathrm{mg}, 0.36 \mathrm{mmol}), \mathrm{Et}_{3} \mathrm{~N}(1 \mathrm{~mL}, 7.2 \mathrm{mmol})$, and acetic anhydride $(510 \mu \mathrm{L}, 5.4$ mmol) gave 6i (yellow oil, $617.7 \mathrm{mg}, 96 \%, \mathrm{R}_{f} 05$, EtOAc/hexane $=3 / 7$ ).

${ }^{1} \mathbf{H}$ NMR $\left(400 \mathrm{MHz}, \mathrm{CDCl}_{3}\right) \delta 7.36(\mathrm{dd}, J=1.6,4.8 \mathrm{~Hz}, 1 \mathrm{H}), 7.27(\mathrm{~d}, J=4.0 \mathrm{~Hz}, 1 \mathrm{H}), 7.00(\mathrm{dd}, \mathrm{J}$ $=3.6,5.2 \mathrm{~Hz}, 1 \mathrm{H}), 6.68(\mathrm{~d}, J=2.0 \mathrm{~Hz}, 1 \mathrm{H}), 2.69(\mathrm{~s}, J=2.0 \mathrm{~Hz}, 1 \mathrm{H}), 2.12(\mathrm{~s}, 3 \mathrm{H})$.<smiles>C#CC(OC(C)=O)C1CCCCC1</smiles>

\section{1-Cyclohexylprop-2-ynyl acetate (6j, CAS: 16169-86-1) ${ }^{[13]}$}

Following the general procedure (GP-2); reaction of S1j (481.0 mg, $3.48 \mathrm{mmol}$ ), N,N-dimethyl-4aminopyridine $(42.5 \mathrm{mg}, 0.35 \mathrm{mmol}), \mathrm{Et}_{3} \mathrm{~N}(970 \mu \mathrm{L}, 6.96 \mathrm{mmol})$, and acetic anhydride $(494 \mu \mathrm{L}$, $5.2 \mathrm{mmol}$ ) gave $\mathbf{6 j}$ (colorless oil, $489.2 \mathrm{mg}$, 78\% for 2 steps, $\mathrm{R}_{f} 047$, EtOAc/hexane $=3 / 7$ ).

${ }^{1} \mathbf{H}$ NMR $\left(400 \mathrm{MHz}, \mathrm{CDCl}_{3}\right) \delta 5.20(\mathrm{dd}, J=2.0,6.0 \mathrm{~Hz}, 1 \mathrm{H}), 2.44(\mathrm{~d}, J=1.6 \mathrm{~Hz}, 1 \mathrm{H}), 2.10(\mathrm{~s}$, $3 \mathrm{H}), 1.91-1.63(\mathrm{~m}, 6 \mathrm{H}), 1.32-1.04(\mathrm{~m}, 5 \mathrm{H})$.<smiles>C#CC1(OC(C)=O)CCCC1</smiles>

\section{1-Ethynylcyclopentyl acetate (6k, CAS: 36193-52-9) ${ }^{[12]}$}

Following the general procedure (GP-2); reaction of crude S1k $(5 \mathrm{mmol})$, Following the general procedure (GP-2); reaction of crude $\mathbf{S 1 j}$ (3.48 mmol), N,N-dimethyl-4-aminopyridine (42.5 mg, $0.35 \mathrm{mmol}), \mathrm{Et}_{3} \mathrm{~N}(970 \mu \mathrm{L}, 6.96 \mathrm{mmol})$, and acetic anhydride $(494 \mu \mathrm{L}, 5.2 \mathrm{mmol})$ gave $\mathbf{6 k}$ (colorless oil, $232.1 \mathrm{mg}, 31 \%$ for 2 steps, $\mathrm{R}_{f} 057$, EtOAc/hexane $=3 / 7$ ).

${ }^{1} \mathbf{H}$ NMR $\left(400 \mathrm{MHz}, \mathrm{CDCl}_{3}\right) \delta 2.56(\mathrm{~s}, 1 \mathrm{H}), 2.26-2.11(\mathrm{~m}, 4 \mathrm{H}), 2.04(\mathrm{~s}, 3 \mathrm{H}), 1.79-1.71(\mathrm{~m}, 4 \mathrm{H})$.

\section{Preparation of 1-phenyl-3-(tributylstannyl)prop-2-yn-1-yl acetate (1a)}

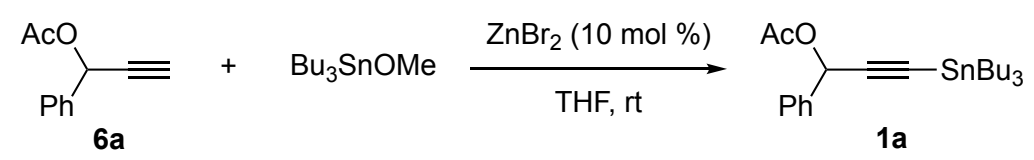

A procedure reported by Baba and Yasuda was used to prepare 1a. ${ }^{[14][15]}$

To a solution of corresponding propargyl acetate $\mathbf{6 a}(10 \mathrm{mmol})$ and $\mathrm{ZnBr}_{2}(225.19 \mathrm{mg}, 1.0 \mathrm{mmol})$ in THF $(20 \mathrm{~mL})$ was added tributyltin methoxide $(3.5 \mathrm{~mL}, 12 \mathrm{mmol})$ at $0{ }^{\circ} \mathrm{C}$ under argon 
atmosphere. After stirring at room temperature for 4 hours, the reaction mixture was quenched with water. The aqueous phase was extracted with diethyl ether $(2 \times 30 \mathrm{~mL})$, and the combined organic extracts were washed with brine $(30 \mathrm{~mL})$. After the organic layer was dried over $\mathrm{MgSO}_{4}$, the solvent was removed under reduced pressure. The residue was purified by column chromatography $\left(10 \% \mathrm{w} / \mathrm{w} \mathrm{K}_{2} \mathrm{CO}_{3}\right.$-neutral silica gel, eluent: EtOAc/hexane $=1 / 19$ with $\left.5 \% \mathrm{Et}_{3} \mathrm{~N}\right)$. Alkynylstannanes 1a was slightly decomposed during the purification by column chromatography. The original paper by $\mathrm{Baba}^{[15]}$ used distillation to purify alkynylstannanes. The isolated alkynylstannane 1a decomposes slowly after several days under air even when refrigerated. However, alkynylstannanes 1a can be stored in fridge for several days under argon atmosphere without significant decomposition.

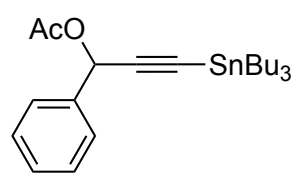

The spectral data of 1-phenyl-3-(tributylstannyl)prop-2-yn-1-yl acetate (1a, cas: 2136264-56-5) are consistent with those of the literature. ${ }^{[14]}$

1-Phenyl-3-(tributylstannyl)prop-2-yn-1-yl acetate (1a): Purified by silica gel column chromatography $\left(29 \%\right.$ yield, $\mathrm{R}_{f} 0.64$, EtOAc/hexane $\left.=1 / 5\right)$. Colorless oil, ${ }^{1} \mathbf{H} \mathbf{~ N M R}\left(\mathrm{CDCl}_{3}, 400\right.$ MHz) $\delta 7.55(\mathrm{dd}, J=1.2,8 \mathrm{~Hz}, 2 \mathrm{H}), 7.40-7.31(\mathrm{~m}, 3 \mathrm{H}), 6.50(\mathrm{~s}, 1 \mathrm{H}), 2.09(\mathrm{~s}, 3 \mathrm{H}), 1.60-1.52(\mathrm{~m}$, $6 \mathrm{H}), 1.40-1.26(\mathrm{~m}, 6 \mathrm{H}), 1.01(\mathrm{t}, J=7.6 \mathrm{~Hz}, 6 \mathrm{H}), 0.89(\mathrm{t}, J=7.6 \mathrm{~Hz}, 9 \mathrm{H}) ;{ }^{13} \mathbf{C} \mathbf{N M R}\left(\mathrm{CDCl}_{3}, 100\right.$ $\mathrm{MHz}) \delta 169.9,137.7,128.8,128.6,128.0,105.9,91.7,66.3,29.0\left(J_{S n-C}=22.9 \mathrm{~Hz}\right), 27.1\left(J_{S n-C}=\right.$ $59.1 \mathrm{~Hz}), 21.3,13.8,11.3\left(J_{S n(119)-C}=380.4, J_{S n(117)-C}=363.3 \mathrm{~Hz}\right)$.

\section{General procedure for three-component reaction using 1a, 3a, and 4- dimethylaminobenzaldehyde}

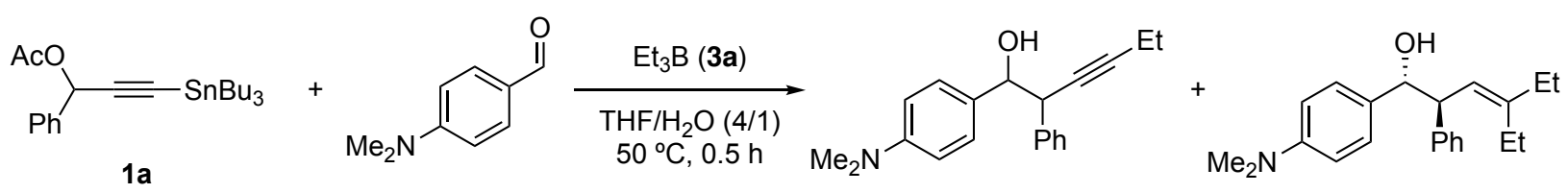

A $10 \mathrm{~mL}$ two neck round-bottom flask was charged with $1 \mathrm{a}(231.62 \mathrm{mg}, 0.5 \mathrm{mmol})$ and THF (2 $\mathrm{mL})$. The mixture was stirred at room temperature, and 4-dimethylaminobenzaldehyde (179.1 $\mathrm{mg}$, $1.2 \mathrm{mmol}), \mathrm{Et}_{3} \mathrm{~B}(3 \mathrm{a})(1.2 \mathrm{mmol}, 1.0 \mathrm{M}$ hexane solution), and distilled water $(0.5 \mathrm{~mL})$ was then added. The reaction mixture was stirred at $50{ }^{\circ} \mathrm{C}$ in an oil bath for 30 minutes under argon atmosphere. Upon completion of the reaction, the reaction mixture was diluted with $\mathrm{Et}_{2} \mathrm{O}(15 \mathrm{~mL})$ and washed with saturated $\mathrm{NH}_{4} \mathrm{Cl}(2 \times 10 \mathrm{~mL})$, saturated $\mathrm{NaHCO}_{3}(2 \times 10 \mathrm{~mL})$, and brine $(2 \times 10$ $\mathrm{mL})$. The combined organic layers were dried over $\mathrm{MgSO}_{4}$ and concentrated. The residue was purified by silica gel chromatography to give 1-(4-dimethylaminophenyl)-2-phenyl-3-hexyn-1-ol (yellow oil, $14.6 \mathrm{mg}, 10 \%, \mathrm{R}_{f} 0.56, \mathrm{EtOAc} / \mathrm{hexane}^{2} 2 / 3$ ) and 4-ethyl-1-(4-dimethylaminophenyl)2-phenylhex-3-en-1-ol (yellow oil, $102.7 \mathrm{mg}, 70 \%, \mathrm{R}_{f} 0.62$, EtOAc/hexane $=2 / 3$ ).

The diastereomeric ratio of 1-(4-dimethylaminophenyl)-2-phenyl-3-hexyn-1-ol was determined by ${ }^{1} \mathrm{H}$ NMR analysis of the crude reaction mixture $(d r=2.4: 1)$.

syn-1-(4-Dimethylaminophenyl)-2-phenyl-3-hexyn-1-ol

${ }^{1} \mathbf{H}$ NMR $\left(\mathrm{CDCl}_{3}, 400 \mathrm{MHz}\right) \delta 7.32-7.20(\mathrm{~m}, 5 \mathrm{H}),(\mathrm{dd}, J=2.4,6.8 \mathrm{~Hz}, 2 \mathrm{H}), 6.66(\mathrm{dd}, J=2.4,6.8$ $\mathrm{Hz}, 2 \mathrm{H}), 4.73(\mathrm{~d}, J=6.8 \mathrm{~Hz}, 1 \mathrm{H}), 3.91(\mathrm{dt}, J=6.8,2.4 \mathrm{~Hz}, 1 \mathrm{H}), 2.93(\mathrm{~s}, 6 \mathrm{H}), 2.18(\mathrm{dq}, J=2.4,7.2$ $\mathrm{Hz}, 2 \mathrm{H}), 2.09$ (br s, 1H), $1.10(\mathrm{t}, J=7.2 \mathrm{~Hz}, 3 \mathrm{H}) ;{ }^{13} \mathbf{C} \mathbf{N M R}\left(\mathrm{CDCl}_{3}, 100 \mathrm{MHz}\right) \delta 150.4,138.9$, 
129.3, 129.0, 128.4, 127.9, 127.3, 112.0, 86.8, 78.5, 78.0, 47.3, 40.8, 14.2, 12.7; HRMS $\mathrm{m} / z\left(\mathrm{M}^{+}\right)$ calcd for $\mathrm{C}_{20} \mathrm{H}_{23} \mathrm{NO}: 276.1747(\mathrm{M}-\mathrm{OH})^{+}$, found: 276.1757 .

anti-1-(4-Dimethylaminophenyl)-2-phenyl-3-hexyn-1-ol

${ }^{1} \mathbf{H}$ NMR $\left(\mathrm{CDCl}_{3}, 400 \mathrm{MHz}\right) \delta 7.30-7.15(\mathrm{~m}, 5 \mathrm{H}), 7.03(\mathrm{~d}, J=8.4 \mathrm{~Hz}, 2 \mathrm{H}), 6.60(\mathrm{dm}, J=8.4 \mathrm{~Hz}$, 2H), $3.87(\mathrm{~m}, 1 \mathrm{H}), 2.90(\mathrm{~s}, 3 \mathrm{H}), 2.28(\mathrm{dq}, J=2.4,7.6 \mathrm{~Hz}, 2 \mathrm{H}), 1.18(\mathrm{t}, J=7.2 \mathrm{~Hz}, 3 \mathrm{H}) ;{ }^{13} \mathbf{C ~ N M R}$ $\left(\mathrm{CDCl}_{3}, 100 \mathrm{MHz}\right) \delta 150.3,139.0,129.1,128.6,128.2,127.6,127.0,112.1,87.6,78.22,78.16$, $48.1,40.7,14.3,12.7$.

\section{anti-4-Ethyl-1-(4-dimethylaminophenyl)-2-phenylhex-3-en-1-ol}

${ }^{1} \mathbf{H}$ NMR $\left(\mathrm{CDCl}_{3}, 400 \mathrm{MHz}\right) \delta 7.21-7.14(\mathrm{~m}, 2 \mathrm{H}), 7.14-7.03(\mathrm{~m}, 3 \mathrm{H}), 7.00(\mathrm{dm}, J=8.8 \mathrm{~Hz}, 2 \mathrm{H})$, $6.57(\mathrm{dm}, J=8.8 \mathrm{~Hz}, 2 \mathrm{H}), 5.57(\mathrm{~d}, J=10.0 \mathrm{~Hz}, 1 \mathrm{H}), 4.69(\mathrm{~d}, J=7.6 \mathrm{~Hz}, 1 \mathrm{H}), 3.78(\mathrm{dd}, J=7.6$, $10.0 \mathrm{~Hz}, 1 \mathrm{H}), 2.87(\mathrm{~s}, 6 \mathrm{H}), 2.23(\mathrm{br} \mathrm{s}, 1 \mathrm{H}), 2.19-2.01(\mathrm{~m}, 4 \mathrm{H}), 1.03(\mathrm{t}, J=7.2 \mathrm{~Hz}, 3 \mathrm{H}), 0.89(\mathrm{t}, J=$ $7.2 \mathrm{~Hz}, 3 \mathrm{H}) ;{ }^{13} \mathbf{C} \mathbf{N M R}\left(\mathrm{CDCl}_{3}, 100 \mathrm{MHz}\right) \delta 150.1,147.2,142.5,130.3,128.5,128.3,127.7$, 126.2, 122.2, 112.2, 78.0, 53.0, 40.8, 29.6, 23.9, 13.2, 13.0; HRMS $m / z\left(\mathrm{M}^{+}\right)$calcd for $\mathrm{C}_{22} \mathrm{H}_{27} \mathrm{~N}$ : $305.2143\left(\mathrm{M}-\mathrm{OH}_{2}\right)^{+}$, found: 305.2136 .

\section{General procedure for three-component reaction (Table 1)}

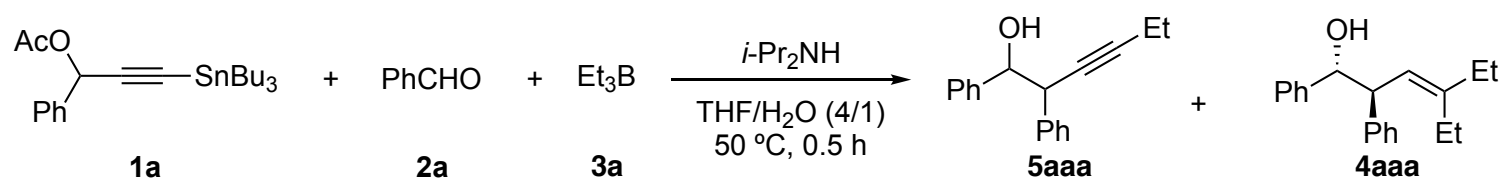

A $10 \mathrm{~mL}$ two neck round-bottom flask was charged with $1 \mathrm{a}(231.62 \mathrm{mg}, 0.5 \mathrm{mmol})$ and THF (2 $\mathrm{mL})$. The mixture was stirred at room temperature under argon atmosphere, and diisopropylamine (121.5 mg, $1.2 \mathrm{mmol}$ ), benzaldehyde (2a) (127.3 mg, 1.2 mmol), Et 3 B (3a) (0.6 mmol, $1.0 \mathrm{M}$ hexane solution), and distilled water $(0.5 \mathrm{~mL})$ was then added. The reaction mixture was stirred at $50{ }^{\circ} \mathrm{C}$ in an oil bath for 30 minutes under argon atmosphere. Upon completion of the reaction, the reaction mixture was diluted with $\mathrm{Et}_{2} \mathrm{O}(15 \mathrm{~mL})$ and washed with saturated $\mathrm{NH}_{4} \mathrm{Cl}(2 \times 10 \mathrm{~mL})$, saturated $\mathrm{NaHCO}_{3}(2 \times 10 \mathrm{~mL})$, and brine $(2 \times 10 \mathrm{~mL})$. The combined organic layers were dried over $\mathrm{MgSO}_{4}$ and concentrated. The residue was purified by silica gel chromatography (synisomer: $\mathrm{R}_{f} 0.35$, anti-isomer: $\mathrm{R}_{f} 0.38, \mathrm{EtOAc} /$ hexane $=1 / 5$ ) to give syn-5aaa (yellow oil, $70.1 \mathrm{mg}$, $56 \%$ ), anti-5aaa (yellow oil, $28.0 \mathrm{mg}, 22 \%$ ), and anti-4aaa (yellow oil, $4.2 \mathrm{mg}, 3 \%, \mathrm{R}_{f} 0.50$, EtOAc/hexane $=1 / 5)$.

The spectral data of anti-1,2-diphenylhex-3-yl-1-ol (anti-5aaa) are consistent with those of the literature. ${ }^{[14]}$

${ }^{1} \mathbf{H}$ NMR $\left(\mathrm{CDCl}_{3}, 400 \mathrm{MHz}\right) \delta 7.26-7.14(\mathrm{~m}, 10 \mathrm{H}), 4.71(\mathrm{dd}, J=3.6,6.8 \mathrm{~Hz}, 1 \mathrm{H}), 3.88(\mathrm{dt}, J=$ $6.8,2.4 \mathrm{~Hz}, 1 \mathrm{H}), 2.77(\mathrm{~d}, J=3.6 \mathrm{~Hz}, 1 \mathrm{H}), 2.28(\mathrm{dq}, J=2.4,7.2 \mathrm{~Hz}, 2 \mathrm{H}), 1.18(\mathrm{t}, J=7.2 \mathrm{~Hz}, 3 \mathrm{H})$; ${ }^{13} \mathrm{C}$ NMR $\left(\mathrm{CDCl}_{3}, 100 \mathrm{MHz}\right) \delta 141.1,138.5,128.6,128.3,128.0,127.8,127.2,126.8,87.9,78.4$, 77.6, 48.2, 14.2, 12.7 .

syn-1,2-Diphenylhex-3-yl-1-ol (syn-5aaa, CAS: 2270914-91-3)

${ }^{1} \mathbf{H}$ NMR $\left(\mathrm{CDCl}_{3}, 400 \mathrm{MHz}\right) \delta 7.29-7.20(\mathrm{~m}, 10 \mathrm{H}), 4.78(\mathrm{dd}, J=3.6,7.2 \mathrm{~Hz}, 1 \mathrm{H}), 3.91(\mathrm{dt}, J=$ $7.2,2.0 \mathrm{~Hz}, 1 \mathrm{H}) 2.30(\mathrm{br} \mathrm{s}, 1 \mathrm{H}), 2.16(\mathrm{dq}, J=2.0,7.2 \mathrm{~Hz}, 2 \mathrm{H}), 1.07$ (t, $J=7.2 \mathrm{~Hz}, 3 \mathrm{H}) ;{ }^{13} \mathbf{C} \mathbf{~ N M R}$ $\left(\mathrm{CDCl}_{3}, 100 \mathrm{MHz}\right) \delta 141.1,138.3,128.9,128.4,127.8$ (two carbon), 127.4, 127.0, 87.2, 78.1(two carbon), 47.2,14.1, 12.6.

The spectral data of anti-4-ethyl-1,2-diphenylhex-3-en-1-ol (anti-4aaa, CAS: 2270914-76-4) are consistent with those of the literature. ${ }^{[14]}$ 
${ }^{1} \mathbf{H}$ NMR $\left(\mathrm{CDCl}_{3}, 400 \mathrm{MHz}\right) \delta 7.26-7.14(\mathrm{~m}, 10 \mathrm{H}), 4.71(\mathrm{dd}, J=3.6,6.8 \mathrm{~Hz}, 1 \mathrm{H}), 3.88(\mathrm{dt}, J=$ $6.8,2.4 \mathrm{~Hz}, 1 \mathrm{H}), 2.77(\mathrm{~d}, J=3.6 \mathrm{~Hz}, 1 \mathrm{H}), 2.28(\mathrm{dq}, J=2.4,7.2 \mathrm{~Hz}, 2 \mathrm{H}), 1.18(\mathrm{t}, J=7.2 \mathrm{~Hz}, 3 \mathrm{H})$; ${ }^{13} \mathbf{C ~ N M R}\left(\mathrm{CDCl}_{3}, 100 \mathrm{MHz}\right) \delta 141.1,138.5,128.6,128.3,128.0,127.8,127.2,126.8,87.9,78.4$, 77.6, 48.3, 14.2, 12.7 .

Table S1. Optimization of Reaction Conditions ${ }^{a}$

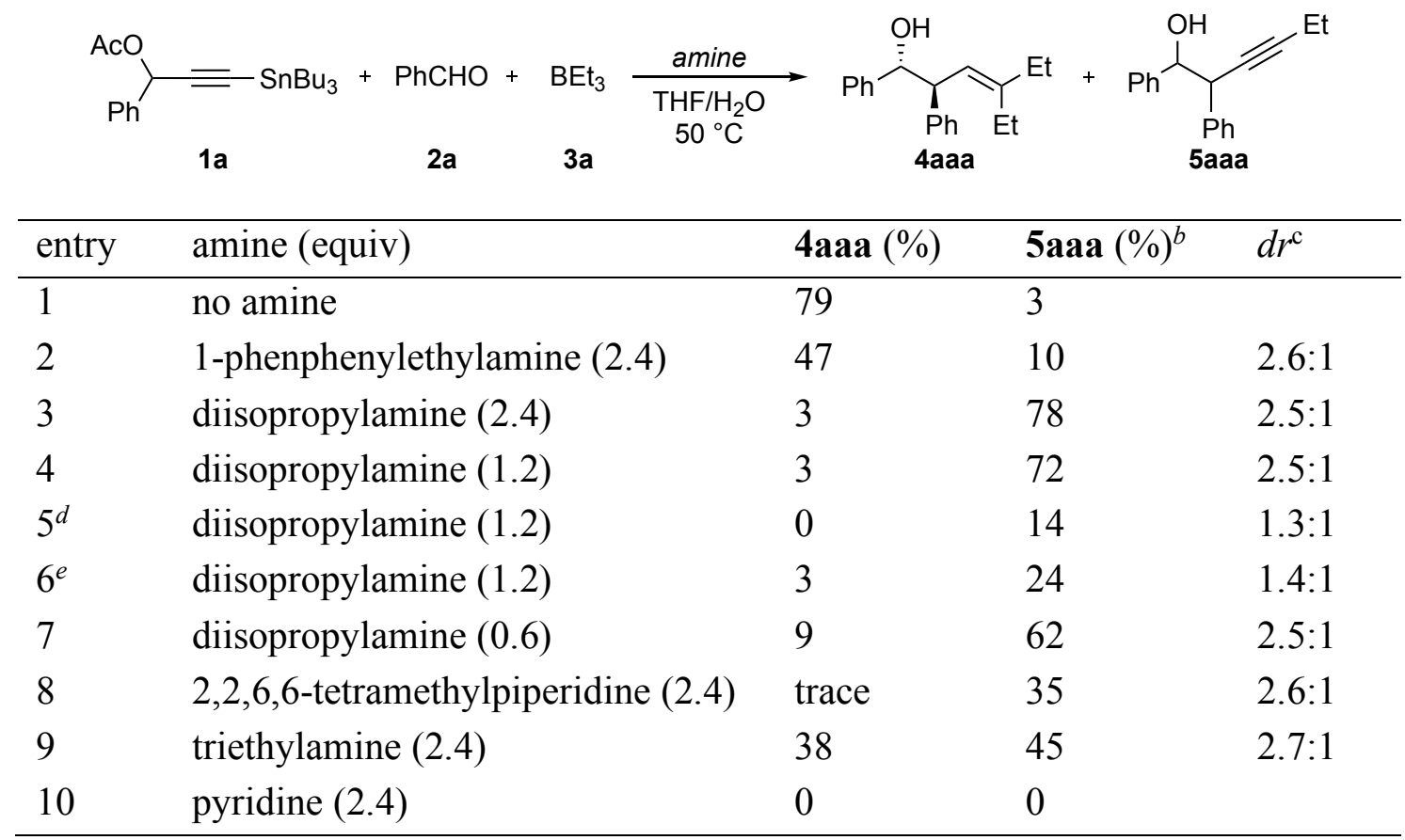

${ }^{a}$ Reaction of 1a $(0.5 \mathrm{mmol})$ with $2 \mathrm{a}(1.2 \mathrm{mmol}), \mathbf{3 a}(1 \mathrm{M}$ in hexane, $0.6 \mathrm{mmol})$, and amine $(1.2 \mathrm{mmol})$ was carried out in $\mathrm{THF} / \mathrm{H}_{2} \mathrm{O}(2.5 \mathrm{~mL}, 4 / 1)$ at $50{ }^{\circ} \mathrm{C}$ under $\mathrm{Ar}$. ${ }^{b}$ syn:anti $=1:>20 .{ }^{c}$ syn isomer is the major product. ${ }^{d}$ no $\mathrm{H}_{2} \mathrm{O}$ was used. ${ }^{e}$ Reaction was performed under $\mathrm{O}_{2}$.

\section{General procedure for three-component reaction using a single flask procedure} (Scheme 2)

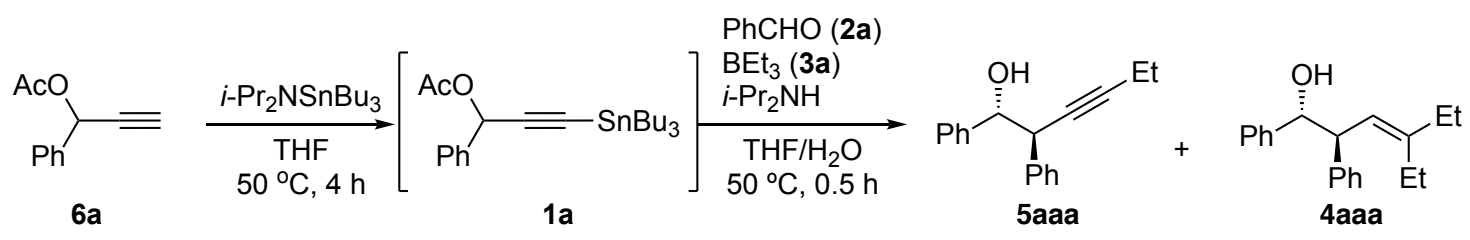

Preparation of (diisopropylamino)tributylstannane: This compound was prepared by modification of a literature procedure. ${ }^{[16]}$

Diisopropylamine $(850 \mu \mathrm{L}, 6.0 \mathrm{mmol})$ was diluted with dry THF $(3.3 \mathrm{~mL})$ and cooled to $-78{ }^{\circ} \mathrm{C}$. $n$-BuLi (1.6 M in hexanes, $3.7 \mathrm{~mL}, 5.9 \mathrm{mmol})$ was added dropwise under an argon atmosphere. Upon completion of the addition, the mixture was warmed to room temperature and stirred for 1 hour. A solution of tributyltin chloride $(1.62 \mathrm{~mL}, 6.0 \mathrm{mmol})$ in dry THF $(1.5 \mathrm{~mL})$ was added to the reaction mixture at room temperature, causing an immediate color change to milky white. After it 
was heated to $50{ }^{\circ} \mathrm{C}$ for 4 hours in an oil bath (pale yellow cloudy suspension), the supernatant was used for the next reaction without further purification.

1-Phenyl-3-(tributylstannyl)-2-propyn-1-yl acetate (1a): This compound was prepared in situ by modification of a published procedure. ${ }^{[17][18]}$

(Diisopropylamino)tributylstannane $(0.5 \mathrm{M}$ in THF, $1.2 \mathrm{~mL}, 0.6 \mathrm{mmol})$ was added to a flask charged with $6 \mathbf{6}(87.1 \mathrm{mg}, 0.5 \mathrm{mmol})$ at room temperature under an argon atmosphere, and then it was heated to $50{ }^{\circ} \mathrm{C}$ in an oil bath for 4 hours. After the reaction completed checked with TLC (S.M.: $\mathrm{R}_{f}=0.45,1 \mathrm{a}: \mathrm{R}_{f}=0.61$, EtOAc/hexane $\left.=1 / 5\right)$, diisopropylamine $(85 \mu \mathrm{L}, 0.6 \mathrm{mmol})$, benzaldehyde (2a) (127.4 mg, $1.2 \mathrm{mmol})$, triethylborane (3a) (1 M in hexane, $0.6 \mathrm{~mL}, 0.6 \mathrm{mmol}$ ), and distilled water $(0.3 \mathrm{~mL})$ were successively added to the mixture at room temperature. The reaction mixture was stirred $50{ }^{\circ} \mathrm{C}$ in an oil bath for 30 minutes. Upon completion of the reaction, the reaction mixture was diluted with $\mathrm{Et}_{2} \mathrm{O}(15 \mathrm{~mL})$ and washed with saturated $\mathrm{NH}_{4} \mathrm{Cl}(2 \times 10$ $\mathrm{mL})$, saturated $\mathrm{NaHCO}_{3}(2 \times 10 \mathrm{~mL})$, and brine $(2 \times 10 \mathrm{~mL})$. The combined organic layers were dried over $\mathrm{MgSO}_{4}$ and concentrated. The residue was purified by silica gel chromatography $(10 \%$ $\mathrm{w} / \mathrm{w} \mathrm{K} \mathrm{CO}_{3}$-silica gel, $\mathrm{R}_{f} 0.50$, EtOAc/hexane = 1/5) to give $s y n-5 \mathbf{a a a}$ (yellow oil, $67.6 \mathrm{mg}, 54 \%$, $\mathrm{R}_{f} 0.35$, EtOAc/hexane $=1 / 4$ ) and anti-5aaa (yellow oil, $27.5 \mathrm{mg}, 22 \%, \mathrm{R}_{f} 0.38$, EtOAc/hexane $=$ $1 / 4)$.

\section{General procedure for three-component reaction using a single flask procedure} (Schemes 3. Scope of Aldehydes)

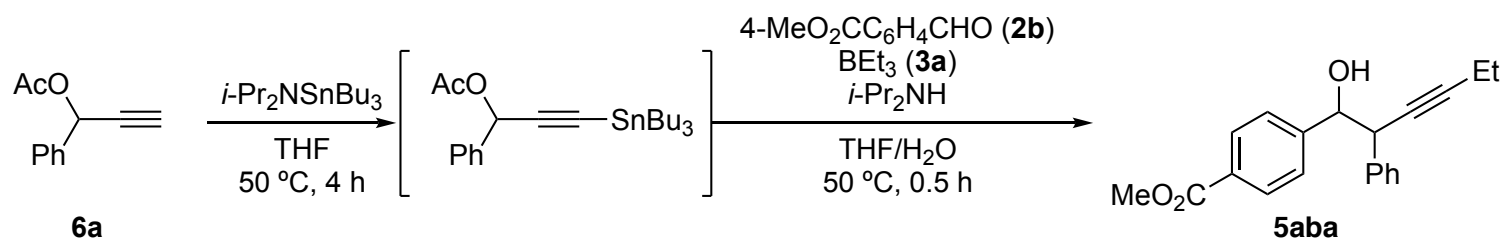

(Diisopropylamino)tributylstannane $(0.5 \mathrm{M}$ in THF, $2.4 \mathrm{~mL}, 1.2 \mathrm{mmol}$ ) was added to a flask charged with 6a $(174.2 \mathrm{mg}, 1.0 \mathrm{mmol})$ at room temperature under an argon atmosphere, and then it was heated to $50{ }^{\circ} \mathrm{C}$ in an oil bath for 30 minutes. After the reaction completed checked with TLC (S.M.: $\mathrm{R}_{f}=0.45,1 \mathrm{a}: \mathrm{R}_{f}=0.61$, EtOAc/hexane $\left.=1 / 5\right)$, diisopropylamine $(170 \mu \mathrm{L}, 1.2 \mathrm{mmol})$, methyl 4-formylbenzoate (2b) $(394.0 \mathrm{mg}, 2.4 \mathrm{mmol})$, triethylborane (3a) (1 M in hexane, $1.2 \mathrm{~mL}$, $1.2 \mathrm{mmol})$, and distilled water $(0.6 \mathrm{~mL})$ were successively added to the mixture at room temperature. The reaction mixture was stirred $50{ }^{\circ} \mathrm{C}$ in an oil bath for 30 minutes. Upon completion of the reaction, the reaction mixture was diluted with $\mathrm{Et}_{2} \mathrm{O}(15 \mathrm{~mL})$ and washed with saturated $\mathrm{NH}_{4} \mathrm{Cl}(2 \times 10 \mathrm{~mL})$, saturated $\mathrm{NaHCO}_{3}(2 \times 10 \mathrm{~mL})$, and brine $(2 \times 10 \mathrm{~mL})$. The combined organic layers were dried over $\mathrm{MgSO}_{4}$ and concentrated. The residue was purified by silica gel chromatography $\left(10 \% \mathrm{w} / \mathrm{w} \mathrm{K} \mathrm{CO}_{3}\right.$-silica gel, $\mathrm{R}_{f} 0.50$, EtOAc/hexane $\left.=1 / 5\right)$ to give syn5aba (yellow solid, $169.3 \mathrm{mg}, 55 \%$ yield, $\mathrm{R}_{f} 0.47$, EtOAc/hexane $\left.=1 / 4\right)$ and anti-5aba $(73.6 \mathrm{mg}$, $24 \%$ yield, $\mathrm{R}_{f} 0.35$, EtOAc/hexane $\left.=1 / 4\right)$.

\section{syn-Methyl 4-(1-hydroxy-2-phenyl-3-hexyn-1-yl)benzoate (syn-5aba)}

${ }^{1} \mathbf{H}$ NMR $\left(\mathrm{CDCl}_{3}, 400 \mathrm{MHz}\right) \delta 7.93(\mathrm{~d}, J=8.4 \mathrm{~Hz}, 2 \mathrm{H}), 7.28-7.21(\mathrm{~m}, 7 \mathrm{H}), 4.85(\mathrm{~d}, J=6.4 \mathrm{~Hz}$, $1 \mathrm{H}), 3.93(\mathrm{dt}, J=6.4,2.0 \mathrm{~Hz}, 1 \mathrm{H}), 3.89$ (s, 3H), 2.47 (s, 1H), 2.17 (dq, $J=2.0,7.6 \mathrm{~Hz}, 2 \mathrm{H}), 1.09$ $(\mathrm{t}, J=7.6 \mathrm{~Hz}, 3 \mathrm{H}) ;{ }^{13} \mathbf{C}$ NMR $\left(\mathrm{CDCl}_{3}, 100 \mathrm{MHz}\right) \delta 167.2,146.1,137.8,129.5,129.0,128.9,128.5$, 127.6, 127.1, 87.7, 77.7, 77.4, 52.2, 47.1, 14.1, 12.5; HRMS $m / z\left(\mathrm{M}^{+}\right)$calcd for $\mathrm{C}_{20} \mathrm{H}_{19} \mathrm{O}_{2}$ : $291.1380(\mathrm{M}-\mathrm{OH})^{+}$, found: 291.1389 . 
anti-Methyl 4-(1-hydroxy-2-phenyl-3-hexyn-1-yl)benzoate (anti-5aba)

anti-5aba was obtained as a mixture of diastereomers.

${ }^{1} \mathbf{H}$ NMR $\left(\mathrm{CDCl}_{3}, 400 \mathrm{MHz}\right) \delta 7.92(\mathrm{~d}, J=8.0 \mathrm{~Hz}, 2 \mathrm{H}), 7.31-7.27(\mathrm{~m}, 5 \mathrm{H}), 7.16-7.13(\mathrm{~m}, 2 \mathrm{H})$, $4.78(\mathrm{dd}, J=6.6 \mathrm{~Hz}, 4.0 \mathrm{~Hz}, 1 \mathrm{H}), 3.90(\mathrm{~s}, 3 \mathrm{H}), 3.87(\mathrm{dt}, J=6.6,2.4 \mathrm{~Hz}, 1 \mathrm{H}), 2.80(\mathrm{~d}, J=4.0 \mathrm{~Hz}$, $1 \mathrm{H}), 2.28(\mathrm{dq}, J=2.4,7.2 \mathrm{~Hz}, 2 \mathrm{H}), 1.16(\mathrm{t}, J=7.2 \mathrm{~Hz}, 3 \mathrm{H}) ;{ }^{13} \mathbf{C} \mathbf{N M R}\left(\mathrm{CDCl}_{3}, 100 \mathrm{MHz}\right) \delta 167.1$, $146.2,137.9,129.3,128.5,128.4,127.5,127.1,126.8,88.3,78.0,77.5,52.2,48.2,14.2,12.7$.<smiles>CCC#CC(O)c1ccc(OC)cc1</smiles>

\section{syn-1-(4-Methoxyphenyl)-2-phenyl-3-hexyn-1-ol (syn-5aca)}

Purified by silica gel column chromatography $\left(131.8 \mathrm{mg}, 47 \%\right.$ yield as a pure form, $\mathrm{R}_{f} 0.25$, EtOAc/hexane $=1 / 4)$. Yellow oil, ${ }^{1} \mathbf{H}$ NMR $\left(\mathrm{CDCl}_{3}, 400 \mathrm{MHz}\right) \delta 7.32-7.23(\mathrm{~m}, 5 \mathrm{H}), 7.17(\mathrm{~d}, J=$ $8.4 \mathrm{~Hz}, 2 \mathrm{H}), 6.81(\mathrm{~d}, J=8.4 \mathrm{~Hz}, 2 \mathrm{H}), 4.77(\mathrm{dd}, J=4.0,7.4 \mathrm{~Hz}, 1 \mathrm{H}), 3.85(\mathrm{dt}, J=7.2,2.4 \mathrm{~Hz}, 1 \mathrm{H})$, $3.80(\mathrm{~s}, 3 \mathrm{H}), 2.18(\mathrm{dq}, J=2.4,7.6 \mathrm{~Hz}, 1 \mathrm{H}), 2.17(\mathrm{~d}, J=4.4 \mathrm{~Hz}, 1 \mathrm{H}), 2.17(\mathrm{~d}, J=4.4 \mathrm{~Hz}, 1 \mathrm{H}), 1.09$ $(\mathrm{t}, J=7.2 \mathrm{~Hz}, 3 \mathrm{H}) ;{ }^{13} \mathbf{C}$ NMR $\left(\mathrm{CDCl}_{3}, 100 \mathrm{MHz}\right) \delta 159.2,138.5,133.3,128.9,128.4,128.2,127.3$, 113.1, 87.0, 78.2, 77.7, 55.3, 47.3, 14.1, 12.6; HRMS: $m / z\left(\mathrm{M}^{+}\right)$calcd for $\mathrm{C}_{19} \mathrm{H}_{18} \mathrm{O}$ : 262.1358 (M$\left.\mathrm{H}_{2} \mathrm{O}\right)^{+}$, found: 262.1351 .

\section{anti-1-(4-Methoxyphenyl)-2-phenyl-3-hexyn-1-ol (anti-5aca)}

Purified by silica gel column chromatography $\left(62.4 \mathrm{mg}, 23 \%\right.$ yield as a pure form, $\mathrm{R}_{f} 0.28$, EtOAc/hexane $=1 / 4)$. Yellow oil, ${ }^{1} \mathbf{H}$ NMR $\left(\mathrm{CDCl}_{3}, 400 \mathrm{MHz}\right) \delta 7.26-7.19(\mathrm{~m}, 3 \mathrm{H}), 7.14(\mathrm{dd}, J=$ 2.0, 7.6 Hz, 2H), $7.08(\mathrm{~d}, J=9.2 \mathrm{~Hz}, 2 \mathrm{H}), 6.77(\mathrm{~d}, J=8.8 \mathrm{~Hz}, 2 \mathrm{H}), 4.66(\mathrm{dd}, J=3.2,6.4 \mathrm{~Hz}, 1 \mathrm{H})$, $3.85(\mathrm{dt}, J=6.4,2.4 \mathrm{~Hz}, 1 \mathrm{H}), 3.77(\mathrm{~s}, 3 \mathrm{H}), 2.74(\mathrm{~d}, J=3.2 \mathrm{~Hz}, 1 \mathrm{H}), 2.29(\mathrm{dq}, J=2.4,7.6 \mathrm{~Hz}, 2 \mathrm{H})$, $1.19(\mathrm{t}, J=7.6 \mathrm{~Hz}, 3 \mathrm{H}) ;{ }^{13} \mathbf{C}$ NMR $\left(\mathrm{CDCl}_{3}, 100 \mathrm{MHz}\right) \delta 159.2,138.5,133.2,128.6,128.3,128.0$, $127.2,113.4,87.8,78.1,77.9,55.3,48.4,14.3,12.7$.

A diastereomixture of 5aca was obtained in 5\% yield (syn:anti $=>10: 1)$.<smiles>CCC#CC(O)C(O)c1cccc(OC)c1</smiles>

\section{syn-1-(3-Methoxyphenyl)-2-phenyl-3-hexyn-1-ol (syn-5ada)}

Purified by silica gel column chromatography $(0.5 \mathrm{mmol}$ scale reaction, $67.3 \mathrm{mg}, 48 \%$ yield as a pure form, $\mathrm{R}_{f} 0.29$, EtOAc/hexane $\left.=1 / 4\right)$. Yellow oil, ${ }^{1} \mathbf{H ~ N M R ~}\left(\mathrm{CDCl}_{3}, 500 \mathrm{MHz}\right) \delta 7.24-7.30(\mathrm{~m}$, $5 \mathrm{H}), 7.18(\mathrm{t}, J=8.0 \mathrm{~Hz}, 1 \mathrm{H}), 6.83(\mathrm{~d}, J=7.5 \mathrm{~Hz}, 1 \mathrm{H}), 6.80(\mathrm{dm}, J=8.0 \mathrm{~Hz}, 1 \mathrm{H}), 6.76(\mathrm{~s}, 1 \mathrm{H})$, $4.80(\mathrm{dd}, J=3.5,6.5 \mathrm{~Hz}, 1 \mathrm{H}), 3.92(\mathrm{dt}, J=6.5,2.5 \mathrm{~Hz}, 1 \mathrm{H}), 3.72(\mathrm{~s}, 3 \mathrm{H}), 2.27(\mathrm{dm}, J=3.5 \mathrm{~Hz}$, $1 \mathrm{H}), 2.18(\mathrm{dq}, J=2.0,7.5 \mathrm{~Hz}, 2 \mathrm{H}), 1.09$ (t, $J=7.5 \mathrm{~Hz}, 3 \mathrm{H}) ;{ }^{13} \mathbf{C} \mathbf{N M R}\left(\mathrm{CDCl}_{3}, 100 \mathrm{MHz}\right) \delta 159.1$, 142.7, 138.3, 129.0, 128.8, 128.4, 127.5, 119.5, 113.8, 112.2, 87.3, 78.1, 78.0, 55.3, 47.2, 14.2, 12.6; HRMS: $m / z\left(\mathrm{M}^{+}\right)$calcd for $\mathrm{C}_{19} \mathrm{H}_{20} \mathrm{O}_{2}: 280.1463$, found: 280.1471 .

Characteristic NMR data of minor diastereomer anti-5ada

Purified by silica gel column chromatography $\left(0.5 \mathrm{mmol}\right.$ scale reaction, $33.6 \mathrm{mg}, 24 \%$ yield, $\mathrm{R}_{f}$ 0.24 , EtOAc/hexane $=1 / 4)$. anti-5ada was obtained as a mixture of diastereomers.

${ }^{1} \mathbf{H}$ NMR $\left(\mathrm{CDCl}_{3}, 500 \mathrm{MHz}\right) \delta 4.68(\mathrm{dd}, J=3.5,6.5 \mathrm{~Hz}, 1 \mathrm{H}), 3.87(\mathrm{dt}, J=6.5,2.5 \mathrm{~Hz}, 1 \mathrm{H}), 3.71$ $(\mathrm{s}, 3 \mathrm{H}), 2.76(\mathrm{~m}, 1 \mathrm{H}), 2.28(\mathrm{dq}, J=2.0,7.5 \mathrm{~Hz}, 2 \mathrm{H}), 1.18(\mathrm{t}, J=7.5 \mathrm{~Hz}, 3 \mathrm{H})$.

${ }^{13} \mathbf{C ~ N M R}\left(\mathrm{CDCl}_{3}, 100 \mathrm{MHz}\right) \delta 159.3,138.5,128.9,128.2,127.2,119.1,113.6,111.9,87.8,78.2$, 77.6, 48.1, 14.2, 12.6 . 
<smiles>CCC#CC(O)c1ccc(Br)cc1</smiles>

\section{syn-1-(4-Bromophenyl)-2-phenyl-3-hexyn-1-ol (syn-5aea)}

Purified by silica gel column chromatography $\left(174.3 \mathrm{mg}, 53 \%\right.$ yield as a pure form, $\mathrm{R}_{f} 0.49$, EtOAc/hexane = 3/7). Yellow oil, ${ }^{1} \mathbf{H}$ NMR $\left(\mathrm{CDCl}_{3}, 400 \mathrm{MHz}\right) \delta 7.37(\mathrm{~d}, J=8.0 \mathrm{~Hz}, 2 \mathrm{H})$, 7.29$7.20(\mathrm{~m}, 5 \mathrm{H}), 7.06(\mathrm{~d}, J=8.0 \mathrm{~Hz}, 2 \mathrm{H}), 4.75(\mathrm{dd}, J=3.6,6.0 \mathrm{~Hz}, 1 \mathrm{H}), 3.88(\mathrm{dt}, J=6.0,2.4 \mathrm{~Hz}$, $1 \mathrm{H}), 2.27(\mathrm{~d}, J=3.6 \mathrm{~Hz}, 1 \mathrm{H}), 2.18(\mathrm{dq}, J=2.4,7.2 \mathrm{~Hz}, 2 \mathrm{H}), 1.10(\mathrm{t}, J=7.2 \mathrm{~Hz}, 3 \mathrm{H}) ;{ }^{13} \mathbf{C ~ N M R}$ $\left(\mathrm{CDCl}_{3}, 100 \mathrm{MHz}\right) \delta 140.0,138.0,130.9,128.9,128.8,128.6,127.6,121.7,87.7,77.6,77.5$ (two carbons), 47.2, 14.1, 12.6; HRMS: $m / z\left(\mathrm{M}^{+}\right)$calcd for $\mathrm{C}_{18} \mathrm{H}_{15} \mathrm{Br}: 310.0357\left(\mathrm{M}-\mathrm{H}_{2} \mathrm{O}\right)^{+}$, found: 310.0364 .

\section{anti-1-(4-Bromophenyl)-2-phenyl-3-hexyn-1-ol (anti-5aea)}

Purified by silica gel column chromatography $\left(67.0 \mathrm{mg}, 21 \%\right.$ yield as a pure form, $\mathrm{R}_{f} 0.53$, EtOAc/hexane = 3/7). Yellow oil, ${ }^{1} \mathbf{H}$ NMR $\left(\mathrm{CDCl}_{3}, 400 \mathrm{MHz}\right) \delta 7.36(\mathrm{~d}, J=8.4 \mathrm{~Hz}, 2 \mathrm{H})$, 7.27$7.22(\mathrm{~m}, 3 \mathrm{H}), 7.14(\mathrm{dd}, J=2.4,8.0 \mathrm{~Hz}, 2 \mathrm{H}), 7.02(\mathrm{~d}, J=8.8 \mathrm{~Hz}, 2 \mathrm{H}), 4.68(\mathrm{dd}, J=3.2,6.8 \mathrm{~Hz}$, $1 \mathrm{H}), 3.82(\mathrm{dt}, J=6.8,2.0 \mathrm{~Hz}, 1 \mathrm{H}), 2.76(\mathrm{~d}, J=3.2 \mathrm{~Hz}, 1 \mathrm{H}), 2.28(\mathrm{dt}, J=2.0,7.2 \mathrm{~Hz}, 2 \mathrm{H}), 1.18(\mathrm{t}$, $J=7.2 \mathrm{~Hz}, 3 \mathrm{H}) ;{ }^{13} \mathbf{C}$ NMR $\left(\mathrm{CDCl}_{3}, 100 \mathrm{MHz}\right) \delta 140.0,138.0,131.1,128.6,128.5,128.5,127.4$, 121.7, 88.2, 77.8, 77.3, 48.3, 14.2, 12.7 .<smiles>CCC#CC(O)c1cccc(I)c1</smiles>

\section{syn-1-(3-Iodophenyl)-2-phenyl-3-hexyn-1-ol (syn-5afa)}

Purified by silica gel column chromatography $(0.5 \mathrm{mmol}$ scale reaction, $88.4 \mathrm{mg}, 47 \%$ yield as a pure form, $\mathrm{R}_{f} 0.35$, EtOAc/hexane $\left.=1 / 4\right)$. Colorless solid, ${ }^{1} \mathbf{H} \mathbf{N M R}\left(\mathrm{CDCl}_{3}, 400 \mathrm{MHz}\right) \delta 7.65(\mathrm{t}, J$ $=1.6 \mathrm{~Hz}, 1 \mathrm{H}), 7.59(\mathrm{dt}, J=1.6,8 \mathrm{~Hz}, 1 \mathrm{H}), 7.33-7.24(\mathrm{~m}, 5 \mathrm{H}), 7.16(\mathrm{~d}, J=8.0 \mathrm{~Hz}, 1 \mathrm{H}), 6.99(\mathrm{t}, J$ $=8.0 \mathrm{~Hz}, 1 \mathrm{H}), 4.71(\mathrm{dd}, J=3.6,6.8 \mathrm{~Hz}, 1 \mathrm{H}), 3.86(\mathrm{dt}, J=6.8,2.4 \mathrm{~Hz}, 1 \mathrm{H}), 2.23(\mathrm{~d}, J=3.6 \mathrm{~Hz}$, $1 \mathrm{H}), 2.19(\mathrm{dq}, J=2.4,7.2 \mathrm{~Hz}, 2 \mathrm{H}), 1.12(\mathrm{t}, J=7.2 \mathrm{~Hz}, 3 \mathrm{H}) ;{ }^{13} \mathbf{C} \mathbf{N M R}\left(\mathrm{CDCl}_{3}, 100 \mathrm{MHz}\right) \delta 143.3$, 137.9, 136.8, 136.2, 129.5, 128.9, 128.6 (two carbon), 127.7, 126.3, 93.7, 87.9, 77.4, 47.3, 14.2, 12.6; HRMS: $m / z\left(\mathrm{M}^{+}\right)$calcd for $\mathrm{C}_{18} \mathrm{H}_{16} \mathrm{I}: 376.0324\left(\mathrm{M}^{+}\right)$, found: 376.0308 .

\section{anti-1-(3-Iodophenyl)-2-phenyl-3-hexyn-1-ol (anti-5afa)}

Purified by silica gel column chromatography $(0.5 \mathrm{mmol}$ scale reaction, $37.6 \mathrm{mg}, 20 \%$ yield as a pure form, $\mathrm{R}_{f} 0.38$, EtOAc/hexane $\left.=1 / 4\right)$. Orange oil, ${ }^{1} \mathbf{H} \mathbf{~ N M R}\left(\mathrm{CDCl}_{3}, 400 \mathrm{MHz}\right) \delta 7.58-7.55(\mathrm{~m}$, $2 \mathrm{H}), 7.29-7.24(\mathrm{~m}, 3 \mathrm{H}), 7.19-7.16(\mathrm{~m}, 2 \mathrm{H}), 7.09-6.94(\mathrm{~m}, 2 \mathrm{H}), 4.66(\mathrm{~d}, J=6.0 \mathrm{~Hz}, 1 \mathrm{H}), 3.84(\mathrm{dt}$, $J=6.0,2.4 \mathrm{~Hz}, 1 \mathrm{H}), 2.71(\mathrm{br} \mathrm{s}, 1 \mathrm{H}), 2.28(\mathrm{dq}, J=2.4,7.2 \mathrm{~Hz}, 2 \mathrm{H}), 1.19(\mathrm{t}, J=7.2 \mathrm{~Hz}, 3 \mathrm{H}) ;{ }^{13} \mathbf{C}$ NMR $\left(\mathrm{CDCl}_{3}, 100 \mathrm{MHz}\right) \delta 143.4,138.0,136.8,135.8,129.6,128.6$ (two carbon), 128.5, 127.5, $126.1,94.0,88.4,77.0,48.2,14.3,12.7$.<smiles>CCC#CC(O)C(c1ccccc1)c1ccc(O)cc1</smiles>

\section{1-(4-Hydroxyphenyl)-2-phenyl-3-hexyn-1-ol (5aga)}

5aga was obtained as a mixture of diastereomers.

Purified by silica gel column chromatography $\left(0.5 \mathrm{mmol}\right.$ scale reaction, $77.2 \mathrm{mg}, 58 \%$ yield, $\mathrm{R}_{f}$ 0.23 , EtOAc/hexane = 1/4). Yellow oil, syn-5aga: ${ }^{1} \mathbf{H}$ NMR $\left(\mathrm{CDCl}_{3}, 400 \mathrm{MHz}\right) \delta 7.33-7.16(\mathrm{~m}$, 4H), 7.13-7.07 (m, 2H), $6.99(\mathrm{~d}, J=8.0 \mathrm{~Hz}, 1 \mathrm{H}), 6.09-6.62(\mathrm{~m}, 2 \mathrm{H}), 4.74(\mathrm{~d}, J=6.8 \mathrm{~Hz}, 1 \mathrm{H})$, 
$3.89(\mathrm{dt}, J=6.8,2.4 \mathrm{~Hz}, 1 \mathrm{H}), 2.16(\mathrm{dq}, J=2.4,7.6 \mathrm{~Hz}, 2 \mathrm{H}), 1.08(\mathrm{t}, J=7.6 \mathrm{~Hz}, 3 \mathrm{H}) ;{ }^{13} \mathbf{C} \mathbf{~ N M R}$ $\left(\mathrm{CDCl}_{3}, 100 \mathrm{MHz}\right) \delta 155.4,138.4,133.1,128.9,128.5,128.4,127.5,114.8,87.3,78.3,77.9,47.3$, 14.2, 12.6; HRMS: $m / z\left(\mathrm{FAB}^{+}\right)$calcd for $\mathrm{C}_{18} \mathrm{H}_{19} \mathrm{O}_{2}: 267.1385(\mathrm{M}+\mathrm{H})$, found: 267.1398 .

anti-5aga: ${ }^{1} \mathbf{H}$ NMR $\left(\mathrm{CDCl}_{3}, 400 \mathrm{MHz}\right) \delta 7.33-7.16(\mathrm{~m}, 5 \mathrm{H}), 7.13-7.07(\mathrm{~m}, 2 \mathrm{H}), 6.09-6.62(\mathrm{~m}$, $2 \mathrm{H}), 4.63(\mathrm{~d}, J=6.8 \mathrm{~Hz}, 1 \mathrm{H}), 3.84(\mathrm{dt}, J=6.4,2.4 \mathrm{~Hz}, 1 \mathrm{H}), 2.28(\mathrm{dq}, J=2.4,7.2 \mathrm{~Hz}, 2 \mathrm{H}), 1.18(\mathrm{t}$, $J=7.2 \mathrm{~Hz}, 3 \mathrm{H})$; Characteristic NMR data of anti-isomer peaks ${ }^{13} \mathbf{C}$ NMR $\left(\mathrm{CDCl}_{3}, 100 \mathrm{MHz}\right) \delta$ $132.9,128.6,128.3,128.2,127.2,115.0,88.0,78.1,77.4,48.3,14.2,12.7$.<smiles>CCC#CC(O)c1ccccc1O</smiles>

1-(2-Hydroxyphenyl)-2-phenyl-3-hexyn-1-ol (5aha)

After silica gel chromatography $\left(10 \% \mathrm{w} / \mathrm{w} \mathrm{K}_{2} \mathrm{CO}_{3}\right.$-silica gel, $\mathrm{R}_{f} 0.45$, EtOAc/hexane = 1/4), 1,3diol group in 5aha was protected as an acetonide due to the instability of 5aha.

5ala was observed in $90 \%{ }^{1} \mathrm{H}$ NMR yield using 1,3,5-trimethoxybenzene as an internal standard.

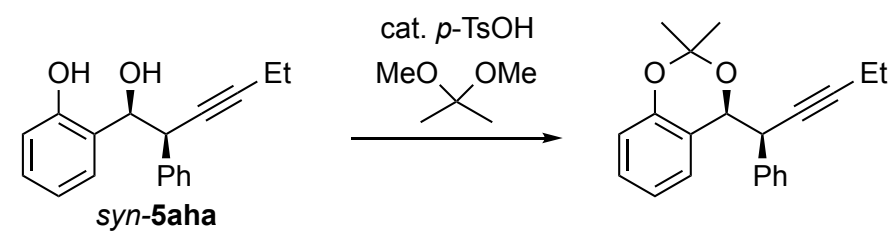

syn-1-(2,2-Dimethyl-4H-benzo[d][1,3]dioxin-4-yl)-1-phenyl-3-pentyn-2-ol

To a solution of 1-(2-hydroxyphenyl)-2-phenyl-3-hexyn-1-ol (5aha) $(219.1 \mathrm{mg}, 0.82 \mathrm{mmol})$ in acetone $(5 \mathrm{~mL})$ was added 2,2-dimethoxypropane $(1.3 \mathrm{~mL}, 17.3 \mathrm{mmol})$ and $p$-toluenesulfonic acid monohydrate $(7 \mathrm{mg}, 0.04 \mathrm{mmol})$. The solution was stirred at room temperature 12 hours, quenched with saturated $\mathrm{NaHCO}_{3}$ and extracted with ethyl acetate. The combined extracts were dried with $\mathrm{MgSO}_{4}$ and concentrated. The residue was purified by silica gel chromatography (133.2 $\mathrm{mg}, 53 \%$ yield, $\mathrm{R}_{f} 0.59$, EtOAc/hexane $\left.=1 / 4\right)$.

${ }^{1} \mathbf{H}$ NMR $\left(\mathrm{CDCl}_{3}, 400 \mathrm{MHz}\right) \delta 7.31-7.29(\mathrm{~m}, 2 \mathrm{H}), 7.26-7.17(\mathrm{~m}, 3 \mathrm{H}), 7.13-7.07(\mathrm{~m}, 2 \mathrm{H}), 6.83(\mathrm{t}$, $J=8.0 \mathrm{~Hz}, 1 \mathrm{H}), 6.71(\mathrm{~d}, J=8.0 \mathrm{~Hz}, 1 \mathrm{H}), 5.19(\mathrm{~d}, J=4.0 \mathrm{~Hz}, 1 \mathrm{H}), 4.15(\mathrm{dt}, J=4.0,2.4 \mathrm{~Hz}, 1 \mathrm{H})$, $2.21(\mathrm{dq}, J=2.4,7.2 \mathrm{~Hz}, 2 \mathrm{H}), 1.53(\mathrm{~s}, 3 \mathrm{H}), 1.40(\mathrm{~s}, 3 \mathrm{H}), 1.11(\mathrm{t}, J=7.2 \mathrm{~Hz}, 3 \mathrm{H}) ;{ }^{13} \mathbf{C} \mathbf{N M R}$ $\left(\mathrm{CDCl}_{3}, 100 \mathrm{MHz}\right) \delta 152.0,138.1,129.4,129.3,127.8,126.9,125.5,122.0,120.2,117.2,99.5$, 86.2, 78.8, 73.8, 44.3, 28.3, 22.1, 14.2, 12.8. HRMS: $m / z\left(\mathrm{M}-\mathrm{CH}_{3}\right)^{+}$calcd for $\mathrm{C}_{20} \mathrm{H}_{19} \mathrm{O}_{2}$ : 291.1380, found: 291.1382 .<smiles>CCC#CC(c1ccccc1)C(O)c1ccccc1OC</smiles>

syn-1-(2-Methoxyphenyl)-2-phenyl-3-hexyn-1-ol (syn-5aia)

Purified by silica gel column chromatography $\left(0.5 \mathrm{mmol}\right.$ scale reaction, $96.7 \mathrm{mg}, 69 \%$ yield, $\mathrm{R}_{f}$ 0.33, EtOAc/hexane = 1/4). Yellow oil, ${ }^{1} \mathbf{H}$ NMR $\left(\mathrm{CDCl}_{3}, 400 \mathrm{MHz}\right) \delta 7.23(\mathrm{~m}, 6 \mathrm{H}), 7.10(\mathrm{dd}, J=$ $1.2,7.2 \mathrm{~Hz}, 1 \mathrm{H}), 6.84(\mathrm{~m}, 2 \mathrm{H}), 5.15(\mathrm{t}, J=6.8 \mathrm{~Hz}, 1 \mathrm{H}), 4.11(\mathrm{dt}, J=6.8,2.4 \mathrm{~Hz}, 1 \mathrm{H}), 3.76(\mathrm{~s}, 3 \mathrm{H})$, $2.83(\mathrm{~d}, J=6.8 \mathrm{~Hz}, 1 \mathrm{H}), 2.19(\mathrm{dq}, J=2.4,7.8 \mathrm{~Hz}, 2 \mathrm{H}), 1.09(\mathrm{t}, J=7.8 \mathrm{~Hz}, 3 \mathrm{H}) ;{ }^{13} \mathbf{C ~ N M R}\left(\mathrm{CDCl}_{3}\right.$, $100 \mathrm{MHz}) \delta 156.6,138.5,129.1,129.0,128.5,128.3,127.9,127.0,120.3,110.2,86.2,79.0,74.2$, 55.3, 44.9, 14.2, 12.7; HRMS: $m / z\left(\mathrm{M}^{+}\right)$calcd for $\mathrm{C}_{19} \mathrm{H}_{19} \mathrm{O}: 263.1430(\mathrm{M}-\mathrm{OH})^{+}$, found: 263.1440. 


\section{General procedure for gram scale reaction using $6 \mathrm{a}, 2 \mathrm{i}$, and $3 \mathrm{a}$}

(Diisopropylamino)tributylstannane $(0.5 \mathrm{M}$ in THF, $14.4 \mathrm{~mL}, 7.2 \mathrm{mmol}$ ) was added to a flask charged with 6a $(1.045 \mathrm{~g}, 6.0 \mathrm{mmol})$ at room temperature under an argon atmosphere, and then it was heated to $50{ }^{\circ} \mathrm{C}$ in an oil bath for 4 hours. After the reaction completed checked with TLC (S.M.: $\mathrm{R}_{f}=0.45$, 1a: $\mathrm{R}_{f}=0.61$, EtOAc/hexane $\left.=1 / 5\right)$, diisopropylamine $(1.0 \mathrm{~mL}, 7.2 \mathrm{mmol})$, 2methoxybenzaldehyde (2i) (1.96 g, $14.4 \mathrm{mmol})$, triethylborane (3a) (1 M in hexane, $7.2 \mathrm{~mL}, 7.2$ $\mathrm{mmol})$, and distilled water $(3.6 \mathrm{~mL})$ were successively added to the mixture at room temperature. The reaction mixture was stirred $50{ }^{\circ} \mathrm{C}$ in an oil bath for 30 minutes. Upon completion of the reaction, the reaction mixture was diluted with $\mathrm{Et}_{2} \mathrm{O}(50 \mathrm{~mL})$ and washed with saturated $\mathrm{NH}_{4} \mathrm{Cl}(2$ $\times 30 \mathrm{~mL})$, saturated $\mathrm{NaHCO}_{3}(2 \times 30 \mathrm{~mL})$, and brine $(2 \times 30 \mathrm{~mL})$. The combined organic layers were dried over $\mathrm{MgSO}_{4}$ and concentrated. The residue was purified by silica gel chromatography $\left(10 \% \mathrm{w} / \mathrm{w} \mathrm{K} \mathrm{CO}_{3}\right.$-silica gel, $\mathrm{R}_{f} 0.50, \mathrm{EtOAc} /$ hexane $\left.=1 / 5\right)$ to give syn-5aia (yellow oil, $1.14 \mathrm{~g}$, $68 \%$ yield, $\mathrm{R}_{f} 0.47$, EtOAc/hexane $\left.=1 / 4\right)$.<smiles>CCC#CC(c1ccccc1)C(O)c1ccc(OC)cc1OC</smiles>

syn-1-(2,4-Dimethoxyphenyl)-2-phenyl-3-hexyn-1-ol (syn-5aja)

Purified by silica gel column chromatography $\left(0.5 \mathrm{mmol}\right.$ scale reaction, $93.1 \mathrm{mg}, 60 \%$ yield, $\mathrm{R}_{f}$ $0.23, \mathrm{EtOAc} /$ hexane $=1 / 4)$. Yellow oil, ${ }^{1} \mathbf{H}$ NMR $\left(\mathrm{CDCl}_{3}, 400 \mathrm{MHz}\right) \delta 7.34-7.17(\mathrm{~m}, 5 \mathrm{H}), 7.02(\mathrm{~d}$, $J=8.4 \mathrm{~Hz}, 1 \mathrm{H}), 6.40-6.36(\mathrm{~m}, 2 \mathrm{H}), 5.09(\mathrm{t}, J=6.0 \mathrm{~Hz}, 1 \mathrm{H}), 4.07(\mathrm{dt}, J=6.0,2.4 \mathrm{~Hz}, 1 \mathrm{H}), 3.78(\mathrm{~s}$, $3 \mathrm{H}), 3.72(\mathrm{~s}, 3 \mathrm{H}), 2.72(\mathrm{~d}, J=6.0 \mathrm{~Hz}, 1 \mathrm{H}), 2.19(\mathrm{dq}, J=2.4,7.8 \mathrm{~Hz}, 2 \mathrm{H}), 1.10(\mathrm{t}, J=7.8 \mathrm{~Hz}, 3 \mathrm{H})$; ${ }^{13} \mathbf{C} \mathbf{N M R}\left(\mathrm{CDCl}_{3}, 100 \mathrm{MHz}\right) \delta 160.3,157.7,138.7,129.1,128.9,127.9,127.0,121.9,103.9,98.2$, 86.2, 79.1, 73.8, 55.43, 55.36, 45.2, 14.3, 12.7; HRMS: $\mathrm{m} / z\left(\mathrm{M}^{+}\right)$calcd for $\mathrm{C}_{20} \mathrm{H}_{21} \mathrm{O}_{2}: 293.1536$ $(\mathrm{M}-\mathrm{OH})^{+}$, found: 293.1548 .<smiles>CCC#CC(O)C(c1ccccc1)c1ccccc1</smiles>

syn-1-(2-Bromophenyl)-2-phenyl-3-hexyn-1-ol (syn-5aka)

Purified by silica gel column chromatography $\left(0.5 \mathrm{mmol}\right.$ scale reaction, $102.0 \mathrm{mg}, 62 \%$ yield, $\mathrm{R}_{f}$ 0.37, EtOAc/hexane = 1/4). Yellow oil, ${ }^{1} \mathbf{H} \mathbf{~ N M R}\left(\mathrm{CDCl}_{3}, 400 \mathrm{MHz}\right) \delta 7.50(\mathrm{~d}, J=7.2 \mathrm{~Hz}, 1 \mathrm{H})$, $7.23-7.18(\mathrm{~m}, 3 \mathrm{H}), 7.15-7.05(\mathrm{~m}, 5 \mathrm{H}), 5.34(\mathrm{dd}, J=2.8,5.6 \mathrm{~Hz}, 1 \mathrm{H}), 4.10(\mathrm{dt}, J=5.6,2.4 \mathrm{~Hz}, 1 \mathrm{H})$, $2.45(\mathrm{~d}, J=2.8 \mathrm{~Hz}, 1 \mathrm{H}), 2.21(\mathrm{dq}, J=2.4,7.2 \mathrm{~Hz}, 2 \mathrm{H}), 1.11(\mathrm{t}, J=7.2 \mathrm{~Hz}, 3 \mathrm{H}) ;{ }^{13} \mathbf{C ~ N M R}\left(\mathrm{CDCl}_{3}\right.$, $100 \mathrm{MHz}) \delta 140.0,137.4,132.3,129.2,129.1,128.7,128.1,127.4,127.2,122.9,86.8,78.2,75.8$, 44.9, 14.1, 12.7; HRMS: $m / z\left(\mathrm{M}^{+}\right)$calcd for $\mathrm{C}_{18} \mathrm{H}_{16} \mathrm{Br}: 311.2220\left(\mathrm{M}-\mathrm{H}_{2} \mathrm{O}\right)^{+}$, found: 311.2227 .<smiles>CCC#CC(c1ccccc1)C1OC(=O)c2ccccc21</smiles>

syn-3-(1-Phenyl-2-pentynyl)-1(3H)-isobenzofuranone (syn-5ala)

Purified by silica gel column chromatography $\left(0.5 \mathrm{mmol}\right.$ scale reaction, $71.8 \mathrm{mg}, 52 \%$ yield, $\mathrm{R}_{f}$ 0.38, EtOAc/hexane = 1/4). Yellow oil, ${ }^{1} \mathbf{H}$ NMR $\left(\mathrm{CDCl}_{3}, 400 \mathrm{MHz}\right) \delta 7.86(\mathrm{~m}, 1 \mathrm{H}), 7.47-7.55$ $(\mathrm{m}, 2 \mathrm{H}), 7.45-7.30(\mathrm{~m}, 5 \mathrm{H}), 6.96(\mathrm{dm}, J=6.0 \mathrm{~Hz}, 1 \mathrm{H}), 5.64(\mathrm{~d}, J=5.6 \mathrm{~Hz}, 1 \mathrm{H}), 4.37(\mathrm{dt}, J=5.6$, $2.0 \mathrm{~Hz}, 1 \mathrm{H}), 2.13(\mathrm{dq}, J=2.0,7.2 \mathrm{~Hz}, 2 \mathrm{H}), 1.02(\mathrm{t}, J=7.2 \mathrm{~Hz}, 3 \mathrm{H}) ;{ }^{13} \mathbf{C ~ N M R}\left(\mathrm{CDCl}_{3}, 100 \mathrm{MHz}\right)$ $\delta 170.2,141.1,135.9,133.3,129.5,128.8,128.7,128.0,127.2,125.4,123.4,88.9,82.9,74.8,43.0$, 13.9, 12.5; HRMS: $m / z\left(\mathrm{M}^{+}\right)$calcd for $\mathrm{C}_{19} \mathrm{H}_{16} \mathrm{O}_{2}: 276.1150$, found: 276.1149 . 


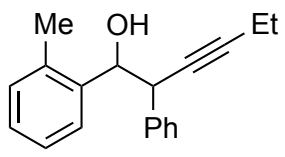

\section{syn-1-(2-Methylphenyl)-2-phenyl-3-hexyn-1-ol (syn-5ama)}

Purified by silica gel column chromatography $\left(0.5 \mathrm{mmol}\right.$ scale reaction, $65.4 \mathrm{mg}, 50 \%$ yield, $\mathrm{R}_{f}$ 0.37, EtOAc/hexane = 1/4). Yellow oil, ${ }^{1} \mathbf{H}$ NMR $\left(\mathrm{CDCl}_{3}, 400 \mathrm{MHz}\right) \delta 7.37(\mathrm{~m}, 1 \mathrm{H}), 7.32-7.22$ $(\mathrm{m}, 5 \mathrm{H}), 7.19-7.13(\mathrm{~m}, 2 \mathrm{H}), 7.09(\mathrm{~m}, 1 \mathrm{H}), 5.10(\mathrm{dd}, J=2.4,7.2 \mathrm{~Hz}, 1 \mathrm{H}), 3.93(\mathrm{dt}, J=7.2,2.4 \mathrm{~Hz}$, $1 \mathrm{H}), 2.27(\mathrm{~s}, 3 \mathrm{H}), 2.19-2.09(\mathrm{~m}, 3 \mathrm{H}), 1.06(\mathrm{t}, J=7.6 \mathrm{~Hz}, 3 \mathrm{H}) ;{ }^{13} \mathbf{C}$ NMR $\left(\mathrm{CDCl}_{3}, 100 \mathrm{MHz}\right) \delta$ 139.6, 138.6, 135.7, 130.0, 129.0, 128.4, 127.6, 127.5, 126.5, 125.9, 86.3, 78.2, 73.8, 46.4, 19.6, 14.0, 12.6; HRMS: $m / z\left(\mathrm{M}^{+}\right)$calcd for $\mathrm{C}_{19} \mathrm{H}_{19}$ : 247.1481, found: 247.1480 .

\section{anti-1-(2-Methylphenyl)-2-phenyl-3-hexyn-1-ol (anti-5ama)}

Purified by silica gel column chromatography $\left(0.5 \mathrm{mmol}\right.$ scale reaction, $29.1 \mathrm{mg}, 22 \%$ yield, $\mathrm{R}_{f}$ 0.42$, EtOAc/hexane $=1 / 4)$.

$7.57(\mathrm{~d}, J=7.6 \mathrm{~Hz}, 1 \mathrm{H}), 7.24-7.13(\mathrm{~m}, 7 \mathrm{H}), 6.98(\mathrm{~d}, J=8.0 \mathrm{~Hz}, 1 \mathrm{H}), 4.93(\mathrm{dd}, J=6.8,3.2 \mathrm{~Hz}$, $1 \mathrm{H}), 3.92(\mathrm{dt}, J=6.8,2.4 \mathrm{~Hz}, 1 \mathrm{H}), 2.76(\mathrm{~d}, J=3.2 \mathrm{~Hz}, 1 \mathrm{H}), 2.32(\mathrm{dq}, J=2.4,7.6 \mathrm{~Hz}, 2 \mathrm{H}), 1.90(\mathrm{~s}$, $3 \mathrm{H}), 1.21(\mathrm{t}, J=7.6 \mathrm{~Hz}, 3 \mathrm{H}) ;{ }^{13} \mathbf{C} \mathbf{~ N M R}\left(\mathrm{CDCl}_{3}, 100 \mathrm{MHz}\right) \delta 139.4,138.4,135.6,130.0,128.5$, $128.3,127.6,127.3,126.4,126.0,88.22,77.5,74.2,47.7,19.0,14.3,12.7$

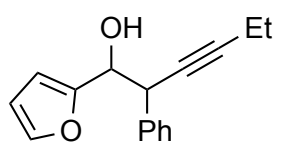

\section{1-(2-Furyl)-2-phenyl-3-hexyn-1-ol (syn-5ana)}

Purified by silica gel column chromatography $(0.5 \mathrm{mmol}$ scale reaction, $81.7 \mathrm{mg}, 68 \%$ yield, syn:anti $=2: 1, \mathrm{R}_{f} 0.31$, EtOAc/hexane $\left.=1 / 4\right)$. Yellow oil, ${ }^{1} \mathbf{H} \mathbf{~ N M R}\left(\mathrm{CDCl}_{3}, 400 \mathrm{MHz}\right) \delta 7.37-$ $7.36(\mathrm{~m}, 1 \mathrm{H}), 7.31(\mathrm{~d}, J=4.0 \mathrm{~Hz}, 4 \mathrm{H}), 7.28-7.22(\mathrm{~m}, 1 \mathrm{H}), 6.31(\mathrm{dd}, J=2.0,3.2 \mathrm{~Hz}, 1 \mathrm{H}), 6.22(\mathrm{~d}$, $J=3.2 \mathrm{~Hz}, 1 \mathrm{H}), 4.86(\mathrm{t}, J=6.8 \mathrm{~Hz}, 1 \mathrm{H}), 4.15(\mathrm{dt}, J=6.8,2.4 \mathrm{~Hz}, 1 \mathrm{H}), 2.22(\mathrm{~d}, J=6.8 \mathrm{H}, 1 \mathrm{H})$, $2.20(\mathrm{dq}, J=2.4,7.6 \mathrm{~Hz}, 2 \mathrm{H}), 1.11(\mathrm{t}, J=7.6 \mathrm{~Hz}, 3 \mathrm{H}) ;{ }^{13} \mathbf{C} \mathbf{N M R}\left(\mathrm{CDCl}_{3}, 100 \mathrm{MHz}\right) \delta 153.9$, $141.9,137.9,128.7,128.5,127.5,110.2,107.7,86.9,77.4,72.1,44.6,14.2,12.6 ;$ HRMS: $\mathrm{m} / z$ $\left(\mathrm{M}^{+}\right)$calcd for $\mathrm{C}_{16} \mathrm{H}_{14} \mathrm{O}: 222.1045\left(\mathrm{M}-\mathrm{H}_{2} \mathrm{O}\right)^{+}$, found: 222.1040 .

anti-5ana was obtained as a mixture of diastereomers.

anti-5ana: ${ }^{1} \mathbf{H}$ NMR $\left(\mathrm{CDCl}_{3}, 400 \mathrm{MHz}\right) \delta 7.33-7.19(\mathrm{~m}, 6 \mathrm{H}), 6.26(\mathrm{dd}, J=2.4,3.2 \mathrm{~Hz}, 1 \mathrm{H}), 6.17$ $(\mathrm{d}, J=3.2 \mathrm{~Hz}, 1 \mathrm{H}), 4.71(\mathrm{t}, J=6.4 \mathrm{~Hz}, 1 \mathrm{H}), 4.19(\mathrm{dt}, J=6.4,2.4 \mathrm{~Hz}, 1 \mathrm{H}), 2.72(\mathrm{~d}, J=6.4 \mathrm{~Hz}, 1 \mathrm{H})$, $2.28(\mathrm{dq}, J=2.4,7.6 \mathrm{~Hz}, 2 \mathrm{H}), 1.17(\mathrm{t}, J=7.6 \mathrm{~Hz}, 3 \mathrm{H})$; Characteristic NMR data of anti-isomer peaks ${ }^{13} \mathbf{C}$ NMR $\left(\mathrm{CDCl}_{3}, 100 \mathrm{MHz}\right) \delta 153.6,142.0,138.4,128.4,128.2,127.4,107.9,88.0,77.1$, $72.4,45.1,14.3,12.7$.<smiles>CCC#CC(O)c1ccccc1</smiles>

\section{syn-1,4-Diphenyl-1-octen-5-yn-3-ol (syn-5aoa)}

Purified by silica gel column chromatography $\left(0.5 \mathrm{mmol}\right.$ scale reaction, $44.2 \mathrm{mg}, 32 \%$ yield, $\mathrm{R}_{f}$ 0.31 , EtOAc/hexane = 1/4). Yellow oil, ${ }^{1} \mathbf{H}$ NMR $\left(\mathrm{CDCl}_{3}, 400 \mathrm{MHz}\right) \delta 7.41-7.21(\mathrm{~m}, 10 \mathrm{H}), 6.55$ $(\mathrm{d}, J=16.0 \mathrm{~Hz}, 1 \mathrm{H}), 6.28(\mathrm{dd}, J=6.0,16.0 \mathrm{~Hz}, 1 \mathrm{H}), 4.42(\mathrm{~m}, 1 \mathrm{H}), 3.90$ (dt, $J=6.0,2.4 \mathrm{~Hz}, 1 \mathrm{H})$, $2.27(\mathrm{dq}, J=2.4,7.2 \mathrm{~Hz}, 2 \mathrm{H}), 2.06(\mathrm{~d}, J=6.0 \mathrm{~Hz}, 1 \mathrm{H}), 1.17(\mathrm{t}, J=7.2 \mathrm{~Hz}, 3 \mathrm{H}) ;{ }^{13} \mathbf{C ~ N M R}\left(\mathrm{CDCl}_{3}\right.$, $100 \mathrm{MHz}) \delta 138.3,137.0,131.9,129.4,128.9,128.8,128.7,127.7,127.5,126.7,87.6,77.5,76.2$, 46.1, 14.4, 12.7; HRMS: $m / z\left(\mathrm{M}^{+}\right)$calcd for $\mathrm{C}_{20} \mathrm{H}_{19}$ : $258.1409\left(\mathrm{M}-\mathrm{H}_{2} \mathrm{O}\right)^{+}$, found: 258.1420 . 


\section{anti-1,4-Diphenyl-1-octen-5-yn-3-ol (anti-5aoa)}

Purified by silica gel column chromatography $\left(0.5 \mathrm{mmol}\right.$ scale reaction, $45.6 \mathrm{mg}, 33 \%$ yield, $\mathrm{R}_{f}$ 0.37, EtOAc/hexane = 1/4). Yellow oil, ${ }^{1} \mathbf{H}$ NMR $\left(\mathrm{CDCl}_{3}, 400 \mathrm{MHz}\right) \delta 7.40-7.20(\mathrm{~m}, 10 \mathrm{H}), 6.54$ $(\mathrm{d}, J=16.0 \mathrm{~Hz}, 1 \mathrm{H}), 6.21(\mathrm{dd}, J=6.0,16.0 \mathrm{~Hz}, 1 \mathrm{H}), 4.39(\mathrm{q}, J=6.0,1 \mathrm{H}), 3.84(\mathrm{dt}, J=6.02 .4 \mathrm{~Hz}$, $1 \mathrm{H}), 2.32(\mathrm{~d}, J=6.0 \mathrm{~Hz}, 1 \mathrm{H}), 2.29(\mathrm{dq}, J=2.4,7.6 \mathrm{~Hz}, 2 \mathrm{H}), 1.18(\mathrm{t}, J=7.6 \mathrm{~Hz}, 3 \mathrm{H}) ;{ }^{13} \mathbf{C} \mathbf{N M R}$ $\left(\mathrm{CDCl}_{3}, 100 \mathrm{MHz}\right) \delta 138.4,136.9,131.6,129.3,128.7,128.6,128.5,127.7,127.4,126.7,87.7$, 77.4, 76.2, 46.4, 14.4, 12.7 .

A diastereomixture of 5aoa was obtained in 4\% isolated yield (syn:anti $=1: 1)$.<smiles>CCC#CC(c1ccccc1)C(O)C(C)C</smiles>

\section{2-Methyl-4-phenyl-1-octen-5-yn-3-ol (5apa)}

5apa was obtained as a mixture of diastereomers.

Purified by silica gel column chromatography $\left(0.5 \mathrm{mmol}\right.$ scale reaction, $74.6 \mathrm{mg}, 69 \%$ yield, $\mathrm{R}_{f}$ 0.55 , EtOAc/hexane $=1 / 4)$. Yellow oil.

syn-5apa: ${ }^{1} \mathbf{H}$ NMR $\left(\mathrm{CDCl}_{3}, 400 \mathrm{MHz}\right) \delta 7.40-7.30(\mathrm{~m}, 4 \mathrm{H}), 7.21-7.15(\mathrm{~m}, 1 \mathrm{H}), 3.86(\mathrm{dt}, J=5.6$, $2.4 \mathrm{~Hz}, 1 \mathrm{H}), 3.30(\mathrm{q}, J=5.6 \mathrm{~Hz}, 1 \mathrm{H}), 2.27(\mathrm{dq}, J=2.4,7.2 \mathrm{~Hz}, 2 \mathrm{H}), 1.93(\mathrm{~d}, J=5.6 \mathrm{~Hz}, 1 \mathrm{H}), 1.77$ (octet, $J=5.6 \mathrm{~Hz}, 1 \mathrm{H}), 1.19-1.12(\mathrm{~m}, 3 \mathrm{H}), 1.02-0.98(\mathrm{~m}, 6 \mathrm{H}) ;{ }^{13} \mathbf{C} \mathbf{N M R}\left(\mathrm{CDCl}_{3}, 100 \mathrm{MHz}\right) \delta$ 140.1, 128.6, 128.2, 127.1, 87.2, 80.5, 77.3, 42.9, 31.4, 20.0, 17.7, 14.4, 12.7; HRMS: $\mathrm{m} / z\left(\mathrm{M}^{+}\right)$ calcd for $\mathrm{C}_{15} \mathrm{H}_{19}$ : $198.1409\left(\mathrm{M}-\mathrm{H}_{2} \mathrm{O}\right)^{+}$, found: 198.1417 .

anti-5apa: ${ }^{1} \mathbf{H}$ NMR $\left(\mathrm{CDCl}_{3}, 400 \mathrm{MHz}\right) \delta 7.28-7.22(\mathrm{~m}, 4 \mathrm{H}), 7.2-7.15(\mathrm{~m}, 1 \mathrm{H}), 3.63(\mathrm{dt}, J=7.8$, $2.4 \mathrm{~Hz}, 1 \mathrm{H}), 3.53(\mathrm{q}, J=3.6 \mathrm{~Hz}, 1 \mathrm{H}), 2.22(\mathrm{dq}, J=2.4,7.2 \mathrm{~Hz}, 2 \mathrm{H}), 2.12(\mathrm{~m}, 1 \mathrm{H}), 1.50(\mathrm{~d}, J=3.6$ $\mathrm{Hz}, 1 \mathrm{H}), 1.14-1.05(\mathrm{~m}, 3 \mathrm{H}), 0.98-0.88(\mathrm{~m}, 6 \mathrm{H})$; Characteristic NMR data of anti-isomer peaks ${ }^{13} \mathbf{C}$ NMR $\left(\mathrm{CDCl}_{3}, 100 \mathrm{MHz}\right) \delta 139.8,128.8,128.6,127.3,85.8,79.8,79.0,43.2,30.3,20.5,15.4$, 14.3 .

General procedure for three-component reaction (Scheme 3, Scope of $\gamma$-Stannylated Propargylic Acetates)

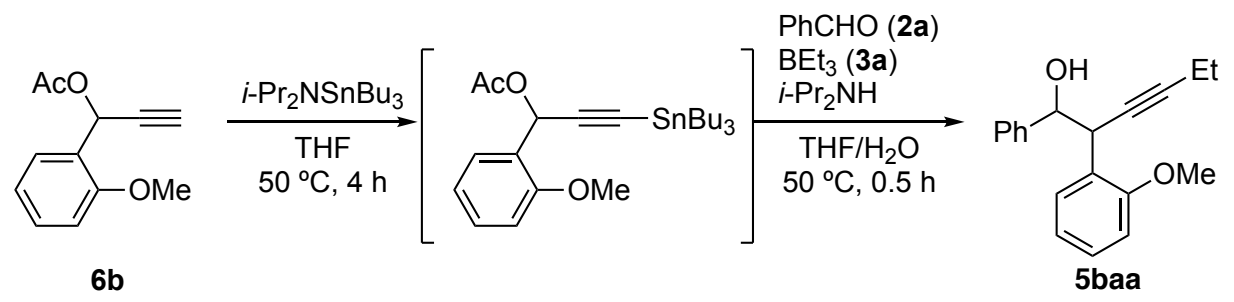

(Diisopropylamino)tributylstannane $(0.5 \mathrm{M}$ in THF, $1.2 \mathrm{~mL}, 0.6 \mathrm{mmol})$ was added to a flask charged with $\mathbf{6 b}(102.1 \mathrm{mg}, 0.5 \mathrm{mmol})$ at room temperature under an argon atmosphere, and then it was heated to $50{ }^{\circ} \mathrm{C}$ in an oil bath for 4 hours. After the reaction completed checked with TLC (S.M.: $\mathrm{R}_{f,}=0.43,1 \mathrm{a}: \mathrm{R}_{f}=0.59$, EtOAc/hexane $\left.=1 / 5\right)$, diisopropylamine $(84 \mu \mathrm{L}, 0.6 \mathrm{mmol})$, benzaldehyde (2a) (127.3 mg, $1.2 \mathrm{mmol}$ ), triethylborane (3a) (1 M in hexane, $0.6 \mathrm{~mL}, 0.6 \mathrm{mmol}$ ), and distilled water $(0.3 \mathrm{~mL})$ were successively added to the mixture at room temperature. The reaction mixture was stirred $50{ }^{\circ} \mathrm{C}$ in an oil bath for 30 minutes. Upon completion of the reaction, the reaction mixture was diluted with $\mathrm{Et}_{2} \mathrm{O}(15 \mathrm{~mL})$ and washed with saturated $\mathrm{NH}_{4} \mathrm{Cl}(2 \times 10$ $\mathrm{mL})$, saturated $\mathrm{NaHCO}_{3}(2 \times 10 \mathrm{~mL})$, and brine $(2 \times 10 \mathrm{~mL})$. The combined organic layers were 
dried over $\mathrm{MgSO}_{4}$ and concentrated. The residue was purified by silica gel chromatography (10\% $\mathrm{w} / \mathrm{w} \mathrm{K}{ }_{2} \mathrm{CO}_{3}$-silica gel, $\mathrm{R}_{f} 0.50$, EtOAc/hexane $=1 / 5$ ) to give $s y n-5$ baa (yellow oil, $76.8 \mathrm{mg}, 55 \%$ yield, $\mathrm{R}_{f} 0.49$, EtOAc/hexane $=1 / 4$ ) and anti-5baa (yellow oil, $25.6 \mathrm{mg}, 19 \%$ yield, $\mathrm{R}_{f} 0.40$, EtOAc/hexane $=1 / 4)$.

\section{syn-1-Phenyl-2-(2-methoxyphenyl)-3-hexyn-1-ol (syn-5baa)}

${ }^{1} \mathbf{H}$ NMR $\left(\mathrm{CDCl}_{3}, 400 \mathrm{MHz}\right) \delta$ 7.24-7.18 (m, $\left.5 \mathrm{H}\right), 7.15-7.11(\mathrm{~m}, 2 \mathrm{H}), 6.88-6.81(\mathrm{~m}, 2 \mathrm{H}), 4.91(\mathrm{t}$, $J=6.0 \mathrm{~Hz}, 1 \mathrm{H}), 4.59(\mathrm{dt}, J=6.0,2.4 \mathrm{~Hz}, 1 \mathrm{H}), 3.74(\mathrm{~s}, 3 \mathrm{H}), 2.65(\mathrm{~d}, J=6.0 \mathrm{~Hz}, 1 \mathrm{H}), 2.19(\mathrm{dq}, J$ $=2.4,7.6 \mathrm{~Hz}, 2 \mathrm{H}), 1.11(\mathrm{t}, J=7.6 \mathrm{~Hz}, 3 \mathrm{H}) ;{ }^{13} \mathbf{C} \mathbf{N M R}\left(\mathrm{CDCl}_{3}, 100 \mathrm{MHz}\right) \delta 156.7,141.4,130.0$, $128.4,127.5,127.4,127.1,126.5,120.7,110.4,86.7,77.9,76.6,55.6,40.0,14.2,12.6$; HRMS: $m / z\left(\mathrm{M}^{+}\right)$calcd for $\mathrm{C}_{19} \mathrm{H}_{19} \mathrm{O}: 263.1430(\mathrm{M}-\mathrm{OH})^{+}$, found: 263.1441 .

anti-1-Phenyl-2-(2-methoxyphenyl)-3-hexyn-1-ol (anti-5baa)

${ }^{1} \mathbf{H}$ NMR $\left(\mathrm{CDCl}_{3}, 400 \mathrm{MHz}\right) \delta 7.54(\mathrm{dd}, J=7.2,1.6 \mathrm{~Hz}, 1 \mathrm{H}), 7.34-7.21(\mathrm{~m}, 6 \mathrm{H}), 6.97(\mathrm{dt}, J=$ 8.0, 0.8 Hz, $1 \mathrm{H}), 6.79(\mathrm{~d}, J=8.0 \mathrm{~Hz}, 1 \mathrm{H}), 4.84(\mathrm{t}, J=4.8 \mathrm{~Hz}, 1 \mathrm{H}), 4.46(\mathrm{dt}, J=4.8,2.4 \mathrm{~Hz}, 1 \mathrm{H})$, $3.70(\mathrm{~s}, 3 \mathrm{H}), 2.68(\mathrm{~d}, J=5.2 \mathrm{~Hz}, 1 \mathrm{H}), 2.25(\mathrm{dq}, J=2.4,7.6 \mathrm{~Hz}, 2 \mathrm{H}), 1.15(\mathrm{t}, J=7.6 \mathrm{~Hz}, 3 \mathrm{H})$; ${ }^{13} \mathrm{C}$ NMR $\left(\mathrm{CDCl}_{3}, 100 \mathrm{MHz}\right) \delta 156.3,142.3,129.7,128.5,127.8,127.4,127.4,126.3,120.7$, $110.5,87.7,77.2,76.1,55.4,41.3,14.3,12.7$.

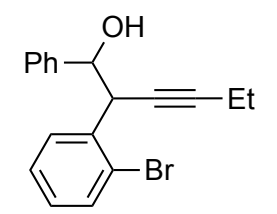

\section{1-Phenyl-2-(2-bromophenyl)-3-hexyn-1-ol (5caa)}

5gaa was obtained as a mixture of diastereomers.

Purified by silica gel column chromatography $\left(140.0 \mathrm{mg}, 85 \%\right.$ yield, syn:anti $=2.6: 1 \mathrm{R}_{f} 0.37$, EtOAc/hexane $=1 / 4)$. Yellow oil.

syn-5caa

${ }^{1} \mathbf{H}$ NMR $\left(\mathrm{CDCl}_{3}, 400 \mathrm{MHz}\right) \delta 7.52(\mathrm{dd}, J=1.2,7.6 \mathrm{~Hz}, 1 \mathrm{H}), 7.37-7.06(\mathrm{~m}, 8 \mathrm{H}), 4.97(\mathrm{t}, J=6.0$ $\mathrm{Hz}, 1 \mathrm{H}), 4.67(\mathrm{dt}, J=5.6,2.4 \mathrm{~Hz}, 1 \mathrm{H}), 2.39(\mathrm{~d}, J=5.6 \mathrm{~Hz}, 1 \mathrm{H}), 2.22(\mathrm{dq}, J=2.4,7.6 \mathrm{~Hz}, 2 \mathrm{H})$, $1.13(\mathrm{t}, J=7.6 \mathrm{~Hz}, 3 \mathrm{H}) ;{ }^{13} \mathbf{C} \mathbf{N M R}\left(\mathrm{CDCl}_{3}, 100 \mathrm{MHz}\right) \delta 140.3,137.7,132.6,131.0,128.9,127.6$, 127.4, 127.3, 126.1, 124.6, 87.7, 76.3, 75.3, 45.8, 14.1, 12.6; HRMS: $\mathrm{m} / \mathrm{z}\left(\mathrm{M}^{+}\right)$calcd for $\mathrm{C}_{18} \mathrm{H}_{15} \mathrm{Br}: 310.0357\left(\mathrm{M}-\mathrm{H}_{2} \mathrm{O}\right)^{+}$, found: 310.0360 .

\section{anti-5caa}

${ }^{1} \mathbf{H}$ NMR $\left(\mathrm{CDCl}_{3}, 400 \mathrm{MHz}\right) \delta 7.73(\mathrm{dd}, J=7.6,2.0 \mathrm{~Hz}, 1 \mathrm{H}), 7.53(\mathrm{dd}, J=1.6 \mathrm{~Hz}, 1 \mathrm{H}), 7.45(\mathrm{~m}$, 2H), 7.37-7.06 (m, 5H), $4.91(\mathrm{dd}, J=4.4,6.4 \mathrm{~Hz}, 1 \mathrm{H}), 4.49(\mathrm{dt}, J=4.4,2.4 \mathrm{~Hz}, 1 \mathrm{H}), 2.47(\mathrm{~d}, J=$ $6.4 \mathrm{~Hz}, 1 \mathrm{H}), 2.25(\mathrm{dq}, J=2.4,8.0 \mathrm{~Hz}, 2 \mathrm{H}), 1.15(\mathrm{t}, J=8.0 \mathrm{~Hz}, 3 \mathrm{H})$; Characteristic NMR data of anti-isomer peaks ${ }^{13} \mathbf{C}$ NMR $\left(\mathrm{CDCl}_{3}, 100 \mathrm{MHz}\right) \delta 141.9,138.5,133.0,131.0,129.0,128.1,127.9$, $127.8,123.9,88.6,77.1,75.9,47.0,14.2,12.7$.

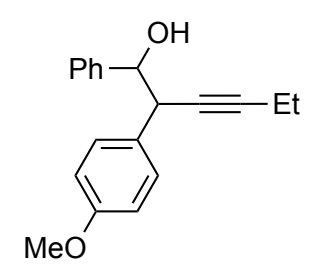

syn-1-Phenyl-2-(4-methoxyphenyl)-3-hexyn-1-ol (syn-5daa)

Purified by silica gel column chromatography $\left(71.5 \mathrm{mg}, 51 \%\right.$ yield, $\mathrm{R}_{f} 0.42$, EtOAc/hexane $\left.=1 / 4\right)$. Yellow oil, ${ }^{1} \mathbf{H}$ NMR $\left(\mathrm{CDCl}_{3}, 400 \mathrm{MHz}\right) \delta 7.37-7.23(\mathrm{~m}, 5 \mathrm{H}), 7.18-7.15(\mathrm{~m}, 2 \mathrm{H}), 6.89-6.80(\mathrm{~m}$, 
2H), $4.67(\mathrm{dd}, J=3.6,7.2 \mathrm{~Hz}, 1 \mathrm{H}), 3.86(\mathrm{dt}, J=7.2,2.4 \mathrm{~Hz}, 1 \mathrm{H}), 3.78(\mathrm{~s}, 3 \mathrm{H}), 2.24(\mathrm{~s}, 1 \mathrm{H}), 2.17$ $(\mathrm{dq}, J=2.4,7.2 \mathrm{~Hz}, 2 \mathrm{H}), 1.08(\mathrm{t}, J=7.2 \mathrm{~Hz}, 3 \mathrm{H}) ;{ }^{13} \mathbf{C} \mathbf{N M R}\left(\mathrm{CDCl}_{3}, 100 \mathrm{MHz}\right) \delta 159.0,141.2$, 130.3, 129.9, 127.82, 127.79, 127.1, 113.8, 87.0, 78.4, 78.2, 55.4, 46.4, 14.2, 12.6; HRMS: $\mathrm{m} / \mathrm{z}$ $\left(\mathrm{M}^{+}\right)$calcd for $\mathrm{C}_{19} \mathrm{H}_{18} \mathrm{O}: 262.1358\left(\mathrm{M}-\mathrm{H}_{2} \mathrm{O}\right)^{+}$, found: 262.1359 .

\section{anti-1-Phenyl-2-(4-methoxyphenyl)-3-hexyn-1-ol (anti-5daa)}

Purified by silica gel column chromatography $\left(35.1 \mathrm{mg}, 25 \%\right.$ yield, $\mathrm{R}_{f} 0.42$, EtOAc/hexane $\left.=1 / 4\right)$. Yellow oil, ${ }^{1} \mathbf{H}$ NMR $\left(\mathrm{CDCl}_{3}, 400 \mathrm{MHz}\right) \delta 7.27-7.20(\mathrm{~m}, 3 \mathrm{H}), 7.19-7.14(\mathrm{~m}, 2 \mathrm{H}), 7.07-7.04(\mathrm{~m}$, $2 \mathrm{H}), 6.78-6.74(\mathrm{~m}, 2 \mathrm{H}), 4.67(\mathrm{dd}, J=6.8,3.6 \mathrm{~Hz}, 1 \mathrm{H}), 3.84(\mathrm{dt}, J=6.8,2.4 \mathrm{~Hz}, 1 \mathrm{H}), 3.76(\mathrm{~s}, 3 \mathrm{H})$, $2.75(\mathrm{~d}, J=3.6 \mathrm{~Hz}, 1 \mathrm{H}), 2.28(\mathrm{dq}, J=2.4,7.2 \mathrm{~Hz}, 2 \mathrm{H}), 1.18(\mathrm{t}, J=7.2 \mathrm{~Hz}, 3 \mathrm{H}) ;{ }^{13} \mathbf{C} \mathbf{N M R}\left(\mathrm{CDCl}_{3}\right.$, $100 \mathrm{MHz}) \delta 158.8,141.1,130.5,129.6,128.0,127.8,126.8,113.7,87.8,78.5,78.0,55.4,47.5$, $14.3,12.7$.<smiles>CCC#CC(c1ccc(C(C)=O)cc1)C(O)c1ccccc1</smiles>

\section{syn-Methyl 4-(1-hydroxyphenylmethyl-2-pentyn-1-yl)benzoate (syn-5eaa)}

Purified by silica gel column chromatography $\left(74.1 \mathrm{mg}, 48 \%\right.$ yield, $\mathrm{R}_{f} 0.30$, EtOAc/hexane $\left.=1 / 4\right)$. Colorless solid, ${ }^{1} \mathbf{H}$ NMR $\left(\mathrm{CDCl}_{3}, 400 \mathrm{MHz}\right) \delta 7.94-7.87(\mathrm{~m}, 2 \mathrm{H}), 7.29-7.22(\mathrm{~m}, 5 \mathrm{H}), 7.21-7.17$ $(\mathrm{m}, 2 \mathrm{H}), 4.87(\mathrm{dd}, J=4.0,6.4 \mathrm{~Hz}, 1 \mathrm{H}), 4.02(\mathrm{dt}, J=6.4,2.4 \mathrm{~Hz}, 1 \mathrm{H}), 3.90(\mathrm{~s}, 3 \mathrm{H}), 2.32(\mathrm{~s}, 1 \mathrm{H})$, $2.20(\mathrm{dq}, J=2.4,7.2 \mathrm{~Hz}, 2 \mathrm{H}), 1.10(\mathrm{t}, J=7.2 \mathrm{~Hz}, 3 \mathrm{H}) ;{ }^{13} \mathbf{C} \mathbf{N M R}\left(\mathrm{CDCl}_{3}, 100 \mathrm{MHz}\right) \delta 167.1$, 143.6, 140.7, 129.5, 129.0 (two carbons), 127.93, 127.85, 126.9, 87.8, 77.8, 77.4, 52.2, 47.1, 14.1, 12.65; HRMS: $m / z\left(\mathrm{M}^{+}\right)$calcd for $\mathrm{C}_{20} \mathrm{H}_{18} \mathrm{O}_{2}: 290.1307$, found: 290.1309 .

anti-Methyl 4-(1-hydroxyphenylmethyl-2-pentyn-1-yl)benzoate (anti-5eaa)

Purified by silica gel column chromatography $\left(27.8 \mathrm{mg}, 18 \%\right.$ yield, $\mathrm{R}_{f} 0.30$, EtOAc/hexane $\left.=1 / 4\right)$. Yellow oil, ${ }^{1} \mathbf{H}$ NMR $\left(\mathrm{CDCl}_{3}, 400 \mathrm{MHz}\right) \delta 7.88(\mathrm{~d}, J=8.4 \mathrm{~Hz}, 2 \mathrm{H}), 7.30-7.19(\mathrm{~m}, 5 \mathrm{H}), 7.15-7.12$ $(\mathrm{m}, 2 \mathrm{H}), 4.72(\mathrm{dd}, J=3.2,6.8 \mathrm{~Hz}, 1 \mathrm{H}), 3.94(\mathrm{dt}, J=6.8,2.4 \mathrm{~Hz}, 1 \mathrm{H}), 3.89(\mathrm{~s}, 3 \mathrm{H}), 2.80(\mathrm{~d}, J=$ $3.2 \mathrm{~Hz}, 1 \mathrm{H}), 2.30(\mathrm{dq}, J=2.4,7.6 \mathrm{~Hz}, 2 \mathrm{H}), 1.19(\mathrm{t}, J=7.6 \mathrm{~Hz}, 3 \mathrm{H}) ;{ }^{13} \mathbf{C} \mathbf{N M R}\left(\mathrm{CDCl}_{3}, 100 \mathrm{MHz}\right)$ $\delta 167.1,143.7,140.6,129.6,129.1,128.7,128.13,128.07,126.7,88.4,78.4,77.2,52.2,48.3,14.2$, 12.7 .

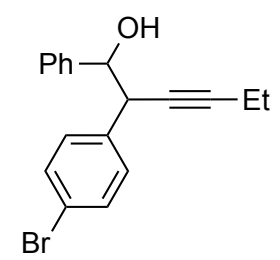

syn-1-Phenyl-2-(4-bromophenyl)-3-hexyn-1-ol (syn-5faa)

Purified by silica gel column chromatography $\left(72.5 \mathrm{mg}, 44 \%\right.$ yield, $\mathrm{R}_{f} 0.44$, EtOAc/hexane $\left.=1 / 4\right)$. Yellow solid, ${ }^{1} \mathbf{H}$ NMR $\left(\mathrm{CDCl}_{3}, 400 \mathrm{MHz}\right) \delta 7.40-7.37(\mathrm{~m}, 2 \mathrm{H}), 7.31-7.24(\mathrm{~m}, 3 \mathrm{H}), 7.22-7.19(\mathrm{~m}$, 2H), 7.11-7.08 (m, 2H), $4.82(\mathrm{dd}, J=4.0,6.4 \mathrm{~Hz}, 1 \mathrm{H}), 3.91(\mathrm{dt}, J=6.4,2.4 \mathrm{~Hz}, 1 \mathrm{H}), 2.24(\mathrm{~d}, J=$ $4.0 \mathrm{~Hz}, 1 \mathrm{H}), 2.18(\mathrm{dq}, J=2.4,7.2 \mathrm{~Hz}, 2 \mathrm{H}), 1.09(\mathrm{t}, J=7.2 \mathrm{~Hz}, 3 \mathrm{H}) ;{ }^{13} \mathbf{C} \mathbf{N M R}\left(\mathrm{CDCl}_{3}, 100 \mathrm{MHz}\right)$ $\delta 140.8,137.3,131.4,130.7,128.00,127.95,126.9,121.4,87.6,77.8,77.7,46.7,14.1,12.6$; HRMS: $m / z\left(\mathrm{M}^{+}\right)$calcd for $\mathrm{C}_{18} \mathrm{H}_{16} \mathrm{Br}: 311.2220\left(\mathrm{M}-\mathrm{H}_{2} \mathrm{O}\right)^{+}$, found: 311.2231 . 
anti-1-Phenyl-2-(4-bromophenyl)-3-hexyn-1-ol (anti-5faa)

Purified by silica gel column chromatography $\left(24.7 \mathrm{mg}, 15 \%\right.$ yield, $\mathrm{R}_{f} 0.44$, EtOAc/hexane $\left.=1 / 4\right)$. Yellow oil, ${ }^{1} \mathbf{H}$ NMR $\left(\mathrm{CDCl}_{3}, 400 \mathrm{MHz}\right) \delta 7.35-7.32(\mathrm{~m}, 2 \mathrm{H}), 7.28-7.22(\mathrm{~m}, 3 \mathrm{H}), 7.16-7.12(\mathrm{~m}$, 2H), 7.01-6.98 (m, 2H), $4.67(\mathrm{dd}, J=2.4,6.8 \mathrm{~Hz}, 1 \mathrm{H}), 3.85(\mathrm{dt}, J=6.8,2.4 \mathrm{~Hz}, 1 \mathrm{H}), 2.75(\mathrm{~s}, 1 \mathrm{H})$, $2.28(\mathrm{dq}, J=2.4,7.2 \mathrm{~Hz}, 2 \mathrm{H}), 1.18(\mathrm{t}, J=7.2 \mathrm{~Hz}, 3 \mathrm{H}) ;{ }^{13} \mathbf{C ~ N M R}\left(\mathrm{CDCl}_{3}, 100 \mathrm{MHz}\right) \delta 140.7$, $137.5,131.3,130.4,128.1,128.0,126.8,121.2,88.3,78.4,77.3,47.7,14.2,12.7$.<smiles>CCC#CC(c1cccc(OC)c1)C(O)c1ccccc1</smiles>

\section{syn-1-Phenyl-2-(3-methoxyphenyl)-3-hexyn-1-ol (syn-5gaa)}

Purified by silica gel column chromatography $\left(70.1 \mathrm{mg}, 50 \%\right.$ yield, $\mathrm{R}_{f} 0.41$, EtOAc/hexane $\left.=1 / 4\right)$. Yellow oil, ${ }^{1} \mathbf{H}$ NMR $\left(\mathrm{CDCl}_{3}, 400 \mathrm{MHz}\right) \delta 7.36-7.18(\mathrm{~m}, 6 \mathrm{H}), 6.87(\mathrm{~d}, J=7.6 \mathrm{~Hz}, 1 \mathrm{H}), 6.80$ $6.76(\mathrm{~m}, 2 \mathrm{H}), 4.79(\mathrm{dd}, J=3.2,7.2 \mathrm{~Hz}, 1 \mathrm{H}), 3.88(\mathrm{dt}, J=7.2,2.4 \mathrm{~Hz}, 1 \mathrm{H}), 3.73(\mathrm{~s}, 3 \mathrm{H}), 2.28(\mathrm{~d}, J$ $=1.6 \mathrm{~Hz}, 1 \mathrm{H}), 2.16(\mathrm{dq}, J=7.2,2.4 \mathrm{~Hz}, 2 \mathrm{H}), 1.07(\mathrm{t}, J=7.2 \mathrm{~Hz}, 3 \mathrm{H}) ;{ }^{13} \mathbf{C ~ N M R}\left(\mathrm{CDCl}_{3}, 100\right.$ MHz) $\delta 159.6,141.1,139.9,129.4,127.8$ (two carbon), 127.1, 121.3, 114.4, 113.1, 87.3, 78.1, 78.0, 55.3, 47.3, 14.1, 12.6; HRMS: $m / z\left(\mathrm{M}^{+}\right)$calcd for $\mathrm{C}_{19} \mathrm{H}_{18} \mathrm{O}: 262.3520\left(\mathrm{M}-\mathrm{H}_{2} \mathrm{O}\right)^{+}$, found: 262.3521.

\section{anti-1-Phenyl-2-(3-methoxyphenyl)-3-hexyn-1-ol (anti-5gaa)}

Purified by silica gel column chromatography $\left(33.7 \mathrm{mg}, 24 \%\right.$ yield, $\mathrm{R}_{f} 0.40$, EtOAc/hexane $\left.=1 / 4\right)$. Yellow oil, ${ }^{1} \mathbf{H}$ NMR $\left(\mathrm{CDCl}_{3}, 400 \mathrm{MHz}\right) \delta 7.28-7.22(\mathrm{~m}, 3 \mathrm{H}), 7.20-7.12(\mathrm{~m}, 3 \mathrm{H}), 6.81-6.73(\mathrm{~m}$, 2H), $6.69(\mathrm{~m}, 1 \mathrm{H}), 4.71(\mathrm{dd}, J=2.8,6.4 \mathrm{~Hz}, 1 \mathrm{H}), 3.86(\mathrm{dt}, J=6.4,2.4 \mathrm{~Hz}, 1 \mathrm{H}), 3.70(\mathrm{~s}, 3 \mathrm{H}), 2.76$ $(\mathrm{d}, J=4.0 \mathrm{~Hz}, 1 \mathrm{H}), 2.28(\mathrm{dq}, J=2.4,7.2 \mathrm{~Hz}, 2 \mathrm{H}), 1.18(\mathrm{t}, J=7.2 \mathrm{~Hz}, 3 \mathrm{H}) ;{ }^{13} \mathbf{C} \mathbf{N M R}\left(\mathrm{CDCl}_{3}, 100\right.$ MHz) $\delta 159.5,141.1,140.0,129.3,128.0,127.8,126.8,120.9,114.2,112.9,88.1,78.4,77.5,55.3$, $48.3,14.3,12.7$.

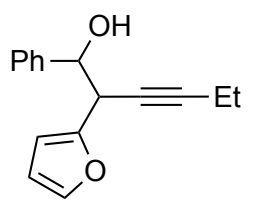

\section{syn-1-Phenyl-2-(2-furyl)-3-hexyn-1-ol (syn-5haa)}

Purified by silica gel column chromatography $\left(56.5 \mathrm{mg}, 47 \%\right.$ yield, $\mathrm{R}_{f} 0.40$, EtOAc/hexane $=3 / 7$ ). Green oil, ${ }^{1} \mathbf{H}$ NMR $\left(\mathrm{CDCl}_{3}, 400 \mathrm{MHz}\right) \delta 7.37-7.25(\mathrm{~m}, 6 \mathrm{H}), 6.31(\mathrm{dd}, J=1.6,3.2 \mathrm{~Hz}, 1 \mathrm{H}), 6.17$ $(\mathrm{d}, J=3.6 \mathrm{~Hz}, 1 \mathrm{H}), 4.97(\mathrm{dd}, J=4.4,6.8 \mathrm{~Hz}, 1 \mathrm{H}), 4.08(\mathrm{dt}, J=6.8,2.4 \mathrm{~Hz}, 1 \mathrm{H}), 2.49(\mathrm{~d}, J=4.4$ $\mathrm{Hz}, 1 \mathrm{H}), 2.15(\mathrm{dq}, J=2.4,7.6 \mathrm{~Hz}, 2 \mathrm{H}), 1.06(\mathrm{t}, J=7.6 \mathrm{~Hz}, 3 \mathrm{H}) ;{ }^{13} \mathbf{C} \mathbf{N M R}\left(\mathrm{CDCl}_{3}, 100 \mathrm{MHz}\right) \delta$ 151.9, 142.2, 140.9, 128.0, 127.9, 126.8, 110.6, 108.1, 86.9, 76.3, 75.1, 41.0, 14.0, 12.5; HRMS: $m / z\left(\mathrm{M}^{+}\right)$calcd for $\mathrm{C}_{16} \mathrm{H}_{14} \mathrm{O}: 222.1045\left(\mathrm{M}-\mathrm{H}_{2} \mathrm{O}\right)^{+}$, found: 222.1056 .

\section{anti-1-Phenyl-2-(2-furyl)-3-hexyn-1-ol (anti-5haa)}

Purified by silica gel column chromatography ( $34.9 \mathrm{mg}, 29 \%$ yield, $\mathrm{R}_{f} 0.40$, EtOAc/hexane $=3 / 7$ ). Green oil, ${ }^{1} \mathbf{H}$ NMR $\left(\mathrm{CDCl}_{3}, 400 \mathrm{MHz}\right) \delta 7.34-7.24(\mathrm{~m}, 6 \mathrm{H}), 6.28(\mathrm{dd}, J=2.8,3.6 \mathrm{~Hz}, 1 \mathrm{H}), 6.16$ $(\mathrm{d}, J=2.8 \mathrm{~Hz}, 1 \mathrm{H}), 4.98(\mathrm{t}, J=4.8 \mathrm{~Hz}, 1 \mathrm{H}), 4.02(\mathrm{dt}, J=4.8,2.4 \mathrm{~Hz}, 1 \mathrm{H}), 2.65(\mathrm{~d}, J=4.8 \mathrm{~Hz}$, $1 \mathrm{H}), 2.25(\mathrm{dq}, J=2.4,7.6 \mathrm{~Hz}, 2 \mathrm{H}), 1.15(\mathrm{t}, J=7.6 \mathrm{~Hz}, 3 \mathrm{H}) ;{ }^{13} \mathbf{C} \mathbf{N M R}\left(\mathrm{CDCl}_{3}, 100 \mathrm{MHz}\right) \delta 151.9$, $142.1,141.2,128.1,127.9,126.4,110.5,107.9,87.4,75.4,74.6,41.9,14.1,12.6$. 


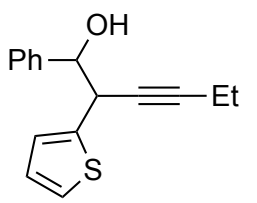

syn-1-Phenyl-2-(2-thienyl)-3-hexyn-1-ol (syn-5iaa)

Purified by silica gel column chromatography $\left(61.5 \mathrm{mg}, 48 \%\right.$ yield, $\mathrm{R}_{f} 0.37$, EtOAc/hexane $\left.=1 / 4\right)$. Yellow oil, ${ }^{1} \mathbf{H}$ NMR $\left(\mathrm{CDCl}_{3}, 400 \mathrm{MHz}\right) \delta 7.39-7.25(\mathrm{~m}, 5 \mathrm{H}), 7.21-7.13(\mathrm{~m}, 1 \mathrm{H}), 6.98-6.91(\mathrm{~m}$, $1 \mathrm{H}), 6.89-6.85(\mathrm{~m}, 1 \mathrm{H}), 4.81(\mathrm{dd}, J=3.2,6.8 \mathrm{~Hz}, 1 \mathrm{H}), 4.17(\mathrm{dt}, J=6.8,2.4 \mathrm{~Hz}, 1 \mathrm{H}), 2.40(\mathrm{~d}, J=$ $3.2 \mathrm{~Hz}, 1 \mathrm{H}), 2.17(\mathrm{dq}, J=2.4,7.6 \mathrm{~Hz}, 2 \mathrm{H}), 1.08(\mathrm{t}, J=7.6 \mathrm{~Hz}, 3 \mathrm{H}) ;{ }^{13} \mathbf{C} \mathbf{N M R}\left(\mathrm{CDCl}_{3}, 100 \mathrm{MHz}\right)$ $\delta 141.5,140.9,128.0$ (two carbons), 126.9, 126.7, 126.5, 125.1, 87.2, 78.0, 77.8, 42.6, 14.0, 12.6; HRMS: $m / z\left(\mathrm{M}^{+}\right)$calcd for $\mathrm{C}_{16} \mathrm{H}_{14} \mathrm{~S}: 238.0816\left(\mathrm{M}-\mathrm{H}_{2} \mathrm{O}\right)^{+}$, found: 238.0810 .

\section{anti-1-Phenyl-2-(2-thienyl)-3-hexyn-1-ol (anti-5iaa)}

Purified by silica gel column chromatography $\left(26.9 \mathrm{mg}, 21 \%\right.$ yield, $\mathrm{R}_{f} 0.37$, EtOAc/hexane $\left.=1 / 4\right)$. Yellow oil, ${ }^{1} \mathbf{H}$ NMR $\left(\mathrm{CDCl}_{3}, 400 \mathrm{MHz}\right) \delta 7.38-7.19(\mathrm{~m}, 5 \mathrm{H}), 7.15-7.14(\mathrm{~m}, 1 \mathrm{H}), 6.93-6.85(\mathrm{~m}$, $1 \mathrm{H}), 6.74(\mathrm{~d}, J=3.6 \mathrm{~Hz}, 1 \mathrm{H}), 4.78(\mathrm{dd}, J=4.0,6.0 \mathrm{~Hz}, 1 \mathrm{H}), 4.18(\mathrm{dt}, J=2.4,6.0 \mathrm{~Hz}, 1 \mathrm{H}), 2.75$ (d, $J=4.0 \mathrm{~Hz}, 1 \mathrm{H}), 2.26(\mathrm{dq}, J=2.4,7.6 \mathrm{~Hz}, 2 \mathrm{H}), 1.17(\mathrm{t}, J=7.6 \mathrm{~Hz}, 3 \mathrm{H}) ;{ }^{13} \mathbf{C ~ N M R}\left(\mathrm{CDCl}_{3}, 100\right.$ $\mathrm{MHz}) \delta 141.7,140.8,128.1,128.0,126.8,126.7,126.0,124.7,87.8,78.3,77.2,43.2,14,1,12.6$.

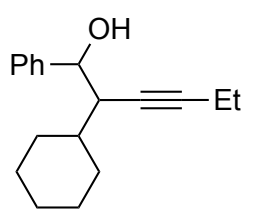

\section{syn-1-Phenyl-2-cyclohexyl-3-hexyn-1-ol (syn-5jaa)}

Purified by silica gel column chromatography $\left(21.8 \mathrm{mg}, 17 \%\right.$ yield, $\mathrm{R}_{f} 0.48$, EtOAc/hexane $\left.=1 / 4\right)$. Yellow oil, ${ }^{1} \mathbf{H}$ NMR $\left(\mathrm{CDCl}_{3}, 400 \mathrm{MHz}\right) \delta 7.42-7.39(\mathrm{~m}, 2 \mathrm{H}), 7.35-7.26(\mathrm{~m}, 3 \mathrm{H}), 4.69(\mathrm{dd}, J=$ $4.8,8.0 \mathrm{~Hz}, 1 \mathrm{H}), 2.63(\mathrm{ddt}, J=8.0,10.4,2.4 \mathrm{~Hz}, 1 \mathrm{H}), 2.14(\mathrm{~d}, J=4.8 \mathrm{~Hz}, 1 \mathrm{H}), 2.08$ (dq, $J=2.4$, $8.0 \mathrm{~Hz}, 2 \mathrm{H}), 1.94-1.82(\mathrm{~m}, 1 \mathrm{H}), 1.74-1.71(\mathrm{~m}, 2 \mathrm{H}), 1.66-1.58(\mathrm{~m}, 3 \mathrm{H}), 1.37-1.11(\mathrm{~m}, 5 \mathrm{H}), 1.01(\mathrm{t}$, $J=8.0 \mathrm{~Hz}, 3 \mathrm{H}) ;{ }^{13} \mathbf{C} \mathbf{N M R}\left(\mathrm{CDCl}_{3}, 100 \mathrm{MHz}\right) \delta 143.1,128.1,127.8,127.1,87.1,77.6,74.2,46.8$, 37.1, 32.4, 28.5, 26.6 (two carbons), 26.3, 14.4, 12.6; HRMS: $m / z\left(\mathrm{M}^{+}\right)$calcd for $\mathrm{C}_{18} \mathrm{H}_{24} \mathrm{O}$ : 256.1827, found: 256.1831 .

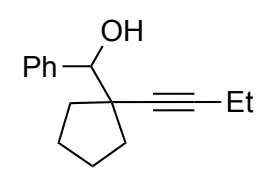

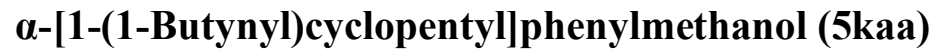

Purified by silica gel column chromatography $\left(27.9 \mathrm{mg}, 23 \%\right.$ yield, $\mathrm{R}_{f} 0.41$, EtOAc/hexane $\left.=1 / 4\right)$. Yellow oil, ${ }^{1} \mathbf{H}$ NMR $\left(\mathrm{CDCl}_{3}, 400 \mathrm{MHz}\right) \delta 7.46-7.43(\mathrm{~m}, 2 \mathrm{H}), 7.34-7.26(\mathrm{~m}, 3 \mathrm{H}), 4.44(\mathrm{~d}, J=6.0$ $\mathrm{Hz}, 1 \mathrm{H}), 2.52(\mathrm{~d}, J=6.0 \mathrm{~Hz}, 1 \mathrm{H}), 2.21(\mathrm{q}, J=7.6 \mathrm{~Hz}, 2 \mathrm{H}), 2.07-1.94(\mathrm{~m}, 1 \mathrm{H}), 1.88-1.43(\mathrm{~m}, 7 \mathrm{H})$, $1.14(\mathrm{t}, J=7.6 \mathrm{~Hz}, 3 \mathrm{H}) ;{ }^{13} \mathrm{C}$ NMR $\left(\mathrm{CDCl}_{3}, 100 \mathrm{MHz}\right) \delta 141.9,127.8$ (two carbons), 127.6, 85.8, 83.0, 79.6, 50.2, 38.8, 36.9, 24.19, 24.17, 14.4, 12.7; HRMS: $m / z\left(\mathrm{M}^{+}\right)$calcd for $\mathrm{C}_{16} \mathrm{H}_{19}$ : 211.1481 $(\mathrm{M}-\mathrm{OH})^{+}$, found: 211.1481 .

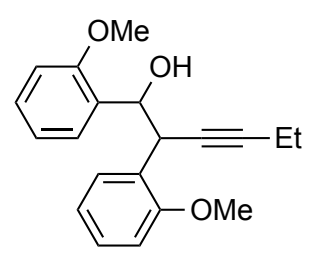

syn-1-(2-Methoxyphenyl)-2-(2-methoxyphenyl)-3-hexyn-1-ol (syn-5bia) 
Purified by silica gel column chromatography $\left(102.4 \mathrm{mg}, 66 \%\right.$ yield, $\mathrm{R}_{f} 0.31$, EtOAc/hexane $=$ 1/4). Yellow oil, ${ }^{1} \mathbf{H}$ NMR $\left(\mathrm{CDCl}_{3}, 400 \mathrm{MHz}\right) \delta 7.38(\mathrm{dd}, J=7.6,1.2 \mathrm{~Hz}, 1 \mathrm{H}), 7.16(\mathrm{tt}, J=7.6$, $2.0 \mathrm{~Hz}, 2 \mathrm{H}), 7.11(\mathrm{~d}, J=7.2 \mathrm{~Hz}, 1 \mathrm{H}), 6.88(\mathrm{t}, J=7.6 \mathrm{~Hz}, 1 \mathrm{H}), 6.81(\mathrm{t}, J=7.6 \mathrm{~Hz}, 1 \mathrm{H}), 6.77(\mathrm{~d}, J=$ $8.4 \mathrm{~Hz}, 1 \mathrm{H}), 6.73(\mathrm{~d}, J=8.4 \mathrm{~Hz}, 1 \mathrm{H}), 5.17(\mathrm{t}, J=6.8 \mathrm{~Hz}, 1 \mathrm{H}), 4.75(\mathrm{dt}, J=6.8,3.2 \mathrm{~Hz}, 1 \mathrm{H}), 3.67$ $(\mathrm{s}, 3 \mathrm{H}), 3.61(\mathrm{~s}, 3 \mathrm{H}), 3.10(\mathrm{~d}, J=6.8 \mathrm{~Hz}, 1 \mathrm{H}), 2.19(\mathrm{dq}, J=2.4,7.6 \mathrm{~Hz}, 2 \mathrm{H}), 1.10(\mathrm{t}, J=7.6 \mathrm{~Hz}$, $3 \mathrm{H}) ;{ }^{13} \mathrm{C} \mathrm{NMR}\left(\mathrm{CDCl}_{3}, 100 \mathrm{MHz}\right) \delta 157.0,156.9,129.70,129.67,128.2$ (two carbons), 128.0, 127.1, 120.4, 119.9, 110.0, 109.9, 85.4, 79.0, 73.4, 55.4, 55.2, 37.7, 14.3, 12.6; HRMS: $m / z\left(\mathrm{M}^{+}\right)$ calcd for $\mathrm{C}_{20} \mathrm{H}_{21} \mathrm{O}_{2}: 292.1463\left(\mathrm{M}-\mathrm{H}_{2} \mathrm{O}\right)^{+}$, found: 292.1469 .

Characteristic NMR data of anti-isomer peaks anti-5bia: ${ }^{1} \mathbf{H}$ NMR $\left(\mathrm{CDCl}_{3}, 400 \mathrm{MHz}\right) \delta 5.09(\mathrm{dd}$, $J=4.8,6.8 \mathrm{~Hz}, 1 \mathrm{H}), 4.59$ (dt, $J=4.8,2.4 \mathrm{~Hz}, 1 \mathrm{H}), 3.76(\mathrm{~s}, 3 \mathrm{H}), 3.66(\mathrm{~s}, 3 \mathrm{H}), 2.93$ (d, $J=6.8 \mathrm{~Hz}$, $1 \mathrm{H}), 2.23(\mathrm{dq}, J=2.4,7.2 \mathrm{~Hz}, 2 \mathrm{H}), 1.13(\mathrm{t}, J=7.2 \mathrm{~Hz}, 3 \mathrm{H})$.

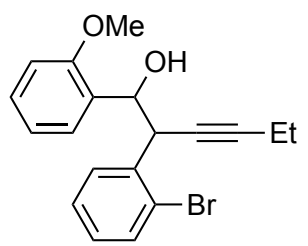

syn-1-(2-Methoxyphenyl)-2-(2-bromophenyl)-3-hexyn-1-ol (syn-5cia)

Purified by silica gel column chromatography $\left(123.9 \mathrm{mg}, 69 \%\right.$ yield, $\mathrm{R}_{f} 0.36$, EtOAc/hexane $=$ 1/4). Yellow oil, ${ }^{1} \mathbf{H}$ NMR $\left(\mathrm{CDCl}_{3}, 400 \mathrm{MHz}\right) \delta 7.46(\mathrm{dt}, J=1.6,7.6 \mathrm{~Hz}, 2 \mathrm{H}), 7.24-7.19(\mathrm{~m}, 3 \mathrm{H})$, $7.07(\mathrm{dt}, J=1.6,7.2 \mathrm{~Hz}, 1 \mathrm{H}), 6.88(\mathrm{t}, J=7.2 \mathrm{~Hz}, 1 \mathrm{H}), 6.76(\mathrm{~d}, J=7.6 \mathrm{~Hz}, 1 \mathrm{H}), 5.13(\mathrm{t}, J=7.6 \mathrm{~Hz}$, $1 \mathrm{H}), 4.80(\mathrm{dt}, J=7.6,2.4 \mathrm{~Hz}, 1 \mathrm{H}), 3.65(\mathrm{~s}, 3 \mathrm{H}), 3.18(\mathrm{~d}, J=8.4 \mathrm{~Hz}, 1 \mathrm{H}), 2.16(\mathrm{dq}, J=2.4,7.2 \mathrm{~Hz}$, $2 \mathrm{H}), 1.08(\mathrm{t}, J=7.2 \mathrm{~Hz}, 3 \mathrm{H}) ;{ }^{13} \mathbf{C} \mathbf{N M R}\left(\mathrm{CDCl}_{3}, 100 \mathrm{MHz}\right) \delta 156.8,138.7,132.3,130.5,129.0$, $128.8,128.6,138.5,127.3,125.2,120.1,110.2,86.5,78.4,74.4,55.2,43.8,14.2,12.6$; HRMS: $m / z\left(\mathrm{M}^{+}\right)$calcd for $\mathrm{C}_{19} \mathrm{H}_{18} \mathrm{BrO}: 340.0463\left(\mathrm{M}-\mathrm{H}_{2} \mathrm{O}\right)^{+}$, found: 340.0465 .

Characteristic NMR data of anti-isomer peaks anti-5cia: ${ }^{\mathbf{1}} \mathbf{H}$ NMR $\left(\mathrm{CDCl}_{3}, 400 \mathrm{MHz}\right) \delta 7.40(\mathrm{~d}, J$ $=7.6 \mathrm{~Hz}, 1 \mathrm{H}), 7.31(\mathrm{dt}, J=0.8,7.2 \mathrm{~Hz}, 1 \mathrm{H}), 7.08(\mathrm{dt}, J=1.2,8.0 \mathrm{~Hz}, 1 \mathrm{H}), 6.93(\mathrm{t}, J=7.2 \mathrm{~Hz}, 1 \mathrm{H})$, $6.82(\mathrm{dd}, J=4.4,8.0 \mathrm{~Hz}, 1 \mathrm{H}), 4.64(\mathrm{dt}, J=2.0,4.4 \mathrm{~Hz}, 1 \mathrm{H}), 3.77(\mathrm{~s}, 3 \mathrm{H}), 2.90(\mathrm{dd}, J=2.0,7.2$ $\mathrm{Hz}, 1 \mathrm{H}), 2.21(\mathrm{dq}, J=2.0,7.6 \mathrm{~Hz}, 2 \mathrm{H}), 1.12(\mathrm{t}, J=7.6 \mathrm{~Hz}, 3 \mathrm{H}) ;{ }^{13} \mathbf{C} \mathbf{N M R}\left(\mathrm{CDCl}_{3}, 100 \mathrm{MHz}\right) \delta$ $156.5,138.7,132.9,131.4,129.4,128.0,123.7,120.3,87.0,72.2,44.5,14.2,12.7$.

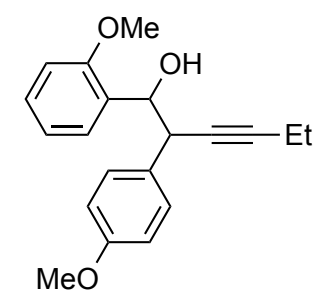

syn-1-(2-Methoxyphenyl)-2-(4-methoxyphenyl)-3-hexyn-1-ol (anti-5dia)

Purified by silica gel column chromatography $\left(111.0 \mathrm{mg}, 72 \%\right.$ yield, $\mathrm{R}_{f} 0.37$, EtOAc/hexane $=$ 1/4). Yellow oil, ${ }^{1} \mathbf{H}$ NMR $\left(\mathrm{CDCl}_{3}, 400 \mathrm{MHz}\right) \delta 7.22(\mathrm{dt}, J=1.6,7.6 \mathrm{~Hz}, 1 \mathrm{H}), 7.09(\mathrm{~d}, J=7.6 \mathrm{~Hz}$, $3 \mathrm{H}), 6.87-6.83(\mathrm{~m}, 2 \mathrm{H}), 6.77(\mathrm{~d}, J=8.4 \mathrm{~Hz}, 2 \mathrm{H}), 5.11(\mathrm{t}, \mathrm{d}, J=6.0 \mathrm{~Hz}, 1 \mathrm{H}), 4.06(\mathrm{dt}, J=6.0,2.4$ $\mathrm{Hz}, 1 \mathrm{H}), 3.79$ (s, $3 \mathrm{H}), 3.77(\mathrm{~s}, 3 \mathrm{H}), 2.78$ (d, $J=6.0 \mathrm{~Hz}, 1 \mathrm{H}), 2.19$ (dq, $J=2.4,7.6 \mathrm{~Hz}, 2 \mathrm{H}), 1.09$ $(\mathrm{t}, J=7.6 \mathrm{~Hz}, 3 \mathrm{H}) ;{ }^{13} \mathbf{C}$ NMR $\left(\mathrm{CDCl}_{3}, 100 \mathrm{MHz}\right) \delta 158.6,156.5,130.5,129.9,129.3,128.4,128.3$, 120.3, 113.3, 110.1, 86.0, 79.3, 74.1, 55.29, 55.26, 14.2, 12.6; HRMS: $m / z\left(\mathrm{M}^{+}\right)$calcd for calcd for $\mathrm{C}_{20} \mathrm{H}_{21} \mathrm{O}_{2}: 292.1463\left(\mathrm{M}-\mathrm{H}_{2} \mathrm{O}\right)^{+}$, found: 292.1457 . 


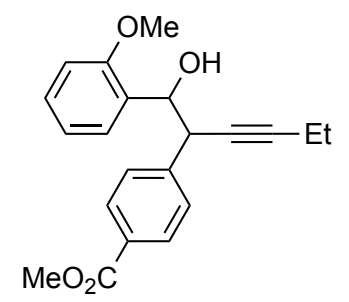

syn-Methyl 4-[1-(2-methoxyphenyl)hydroxymethyl-2-pentyn-1-yl]benzoate (syn-5eia)

Purified by silica gel column chromatography $\left(116.6 \mathrm{mg}, 69 \%\right.$ yield, $\mathrm{R}_{f} 0.23$, EtOAc/hexane $=$ 1/4). Yellow oil, ${ }^{1} \mathbf{H}$ NMR $\left(\mathrm{CDCl}_{3}, 400 \mathrm{MHz}\right) \delta 7.89-7.87(\mathrm{~m}, 2 \mathrm{H}), 7.24-7.19(\mathrm{~m}, 3 \mathrm{H}), 7.05(\mathrm{dd}$, $J=1.2,7.6 \mathrm{~Hz}, 1 \mathrm{H}), 6.85-6.81(\mathrm{~m}, 2 \mathrm{H}), 5.18(\mathrm{t}, J=6.4 \mathrm{~Hz}, 1 \mathrm{H}), 4.19(\mathrm{dt}, J=5.2,2.4 \mathrm{~Hz}, 1 \mathrm{H})$, $3.89(\mathrm{~s}, 3 \mathrm{H}), 3.76(\mathrm{~s}, 3 \mathrm{H}), 2.91(\mathrm{~d}, J=6.4 \mathrm{~Hz}, 1 \mathrm{H}), 2.21(\mathrm{dq}, J=2.4,7.6 \mathrm{~Hz}, 2 \mathrm{H}), 1.11(\mathrm{t}, J=7.6$ $\mathrm{Hz}, 3 \mathrm{H}) ;{ }^{13} \mathrm{C}$ NMR $\left(\mathrm{CDCl}_{3}, 100 \mathrm{MHz}\right) \delta 167.2,156.3,143.9,129.2,129.1,128.74,128.71$, 128.65, 128.2, 120.4, 110.2, 86.9, 78.4, 74.0, 55.3, 55.1, 44.8, 14.2, 12.6 HRMS: $\mathrm{m} / z\left(\mathrm{M}^{+}\right)$calcd for calcd for $\mathrm{C}_{21} \mathrm{H}_{21} \mathrm{O}_{3}: 320.1412\left(\mathrm{M}-\mathrm{H}_{2} \mathrm{O}\right)^{+}$, found: 320.1415 .

Characteristic NMR data of anti-isomer peaks anti-5eia: ${ }^{1} \mathbf{H}$ NMR $\left(\mathrm{CDCl}_{3}, 400 \mathrm{MHz}\right) \delta 5.08(\mathrm{dd}$, $J=7.2,4.8 \mathrm{~Hz}, 1 \mathrm{H}), 4.15(\mathrm{dt}, J=4.8,2.4 \mathrm{~Hz}, 1 \mathrm{H}), 3.89(\mathrm{~s}, 3 \mathrm{H}), 3.70(\mathrm{~s}, 3 \mathrm{H}), 2.88(\mathrm{~d}, J=7.2 \mathrm{~Hz}$, $1 \mathrm{H}), 2.27(\mathrm{dq}, J=2.4,7.2 \mathrm{~Hz}, 2 \mathrm{H}), 1.16(\mathrm{t}, J=7.6 \mathrm{~Hz}, 3 \mathrm{H})$.

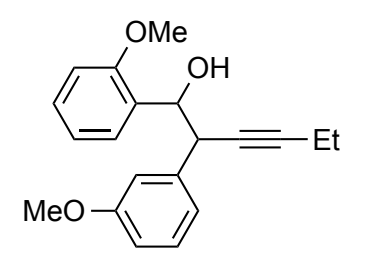

syn-1-(2-Methoxyphenyl)-2-(3-methoxyphenyl)-3-hexyn-1-ol (syn-5gia)

Purified by silica gel column chromatography $\left(111.7 \mathrm{mg}, 72 \%\right.$ yield, $\mathrm{R}_{f} 0.23$, EtOAc/hexane $=$ 1/4). Yellow oil, ${ }^{1} \mathbf{H}$ NMR $\left(\mathrm{CDCl}_{3}, 400 \mathrm{MHz}\right) \delta 7.21(\mathrm{dt}, J=2.0,8.4 \mathrm{~Hz}, 1 \mathrm{H}), 7.16-7.11(\mathrm{~m}, 2 \mathrm{H})$, $6.87-6.79(\mathrm{~m}, 3 \mathrm{H}), 6.76-6.72(\mathrm{~m}, 2 \mathrm{H}), 5.14(\mathrm{t}, J=6.4 \mathrm{~Hz}, 1 \mathrm{H}), 4.09(\mathrm{dt}, J=6.4,2.0 \mathrm{~Hz}, 1 \mathrm{H}), 3.76$ $(\mathrm{s}, 3 \mathrm{H}), 3.69(\mathrm{~s}, 3 \mathrm{H}), 2.83(\mathrm{~d}, J=6.4 \mathrm{~Hz}, 1 \mathrm{H}), 2.18(\mathrm{dq}, J=2.4,7.2 \mathrm{~Hz}, 2 \mathrm{H}), 1.08(\mathrm{t}, J=7.2 \mathrm{~Hz}$, $3 \mathrm{H}) ;{ }^{13} \mathbf{C ~ N M R}\left(\mathrm{CDCl}_{3}, 100 \mathrm{MHz}\right) \delta 159.2,156.6,140.0,129.2,128.8,128.5,128.4,121.5,120.3$, 114.5, 112.7, 110.2, 86.3, 78.9, 74.2, 55.3, 55.2, 44.9, 14.2, 12.6; HRMS: $\mathrm{m} / \mathrm{z}\left(\mathrm{M}^{+}\right)$calcd for calcd for $\mathrm{C}_{20} \mathrm{H}_{21} \mathrm{O}_{2}: 292.1463\left(\mathrm{M}-\mathrm{H}_{2} \mathrm{O}\right)^{+}$, found: 292.1460 .

Characteristic NMR data of anti-isomer peaks anti-5gia: ${ }^{1} \mathbf{H}$ NMR $\left(\mathrm{CDCl}_{3}, 400 \mathrm{MHz}\right) \delta 5.09$ (dd, $J=4.0,6.8 \mathrm{~Hz}, 1 \mathrm{H}), 3.78(\mathrm{~s}, 3 \mathrm{H}), 3.75(\mathrm{~s}, 3 \mathrm{H}), 2.77(\mathrm{~d}, J=6.8 \mathrm{~Hz}, 1 \mathrm{H}), 2.25(\mathrm{dq}, J=2.4,7.6 \mathrm{~Hz}$, $2 \mathrm{H}), 1.15(\mathrm{t}, J=7.6 \mathrm{~Hz}, 3 \mathrm{H})$.

General procedure for three-component reaction (Scheme 4, Scope with Respected to Triorganoboranes)

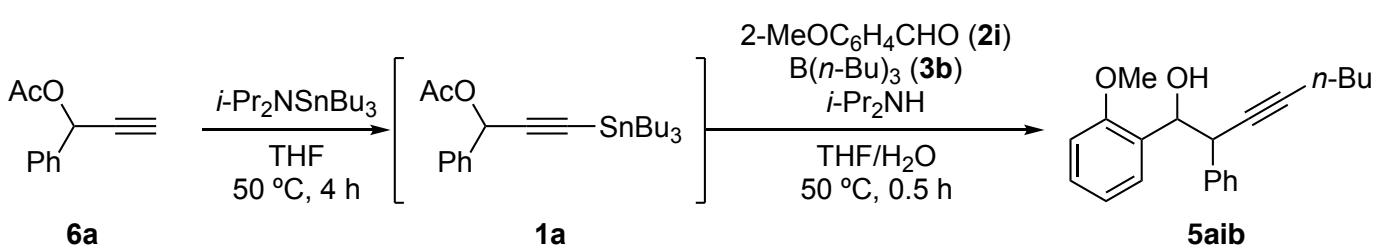

(Diisopropylamino)tributylstannane $(0.5 \mathrm{M}$ in THF, $1.2 \mathrm{~mL}, 0.6 \mathrm{mmol}$ ) was added to a flask charged with $\mathbf{6 a}(87.1 \mathrm{mg}, 0.5 \mathrm{mmol})$ at room temperature under an argon atmosphere, and then it was heated to $50{ }^{\circ} \mathrm{C}$ in an oil bath for 4 hours. After the reaction completed checked with TLC (S.M.: $\mathrm{R}_{f}=0.36,1 \mathrm{a}: \mathrm{R}_{f}=0.60$, EtOAc/hexane $\left.=1 / 5\right)$, diisopropylamine $(84 \mu \mathrm{L}, 0.6 \mathrm{mmol})$, 2- 
methoxybenzaldehyde (2i) (163.4 mg, $1.2 \mathrm{mmol})$, tributylborane (3b) $\left(1 \mathrm{M}\right.$ in $\mathrm{Et}_{2} \mathrm{O}, 0.6 \mathrm{~mL}, 0.6$ $\mathrm{mmol})$, and distilled water $(0.3 \mathrm{~mL})$ were successively added to the mixture at room temperature. The reaction mixture was stirred $50{ }^{\circ} \mathrm{C}$ in an oil bath for 30 minutes. Upon completion of the reaction, the reaction mixture was diluted with $\mathrm{Et}_{2} \mathrm{O}(15 \mathrm{~mL})$ and washed with saturated $\mathrm{NH}_{4} \mathrm{Cl}(2$ $\times 10 \mathrm{~mL})$, saturated $\mathrm{NaHCO}_{3}(2 \times 10 \mathrm{~mL})$, and brine $(2 \times 10 \mathrm{~mL})$. The combined organic layers were dried over $\mathrm{MgSO}_{4}$ and concentrated. The residue was purified by silica gel chromatography $\left(10 \% \mathrm{w} / \mathrm{w} \mathrm{K} \mathrm{CO}_{3}-\right.$ silica gel, $\mathrm{R}_{f} 0.50, \mathrm{EtOAc} /$ hexane $\left.=1 / 5\right)$ to give syn-5aib (colorless oil, 99.6 $\mathrm{mg}, 65 \%$ yield, $\mathrm{R}_{f} 0.40$, EtOAc/hexane $\left.=1 / 4\right)$.

Other trialkylboranes are prepared as follows: To a solution of alkene $(2.25 \mathrm{mmol})$ in THF $(1.5$ $\mathrm{mL})$ was added dropwise a $\mathrm{BH}_{3} \cdot \mathrm{SMe}_{2}(0.75 \mathrm{mmol})$ at $0{ }^{\circ} \mathrm{C}$. After addition, the reaction mixture was warmed to room temperature and stirred for 12 hours. Upon completion of the reaction (checked by NMR), the solvent was removed under reduced pressure to give a trialkylborane, which was used for next reaction without further purification.

\section{syn-1-(2-Methoxyphenyl)-2-phenyl-3-octyn-1-ol (syn-5aib)}

${ }^{1} \mathbf{H}$ NMR $\left(\mathrm{CDCl}_{3}, 400 \mathrm{MHz}\right) \delta 7.27-7.17(\mathrm{~m}, 6 \mathrm{H}), 7.11(\mathrm{dd}, J=1.6,8.0 \mathrm{~Hz}, 1 \mathrm{H}), 6.87-6.82(\mathrm{~m}$, $2 \mathrm{H}), 5.14(\mathrm{t}, J=6.4 \mathrm{~Hz}, 1 \mathrm{H}), 4.13(\mathrm{dt}, J=6.4,2.4 \mathrm{~Hz}, 1 \mathrm{H}), 3.77(\mathrm{~s}, 3 \mathrm{H}), 2.81(\mathrm{~d}, J=6.4 \mathrm{~Hz}, 1 \mathrm{H})$, $2.18(\mathrm{dt}, J=2.4,6.8 \mathrm{~Hz}, 2 \mathrm{H}), 1.47-1.39(\mathrm{~m}, 2 \mathrm{H}), 1.38-1.28(\mathrm{~m}, 2 \mathrm{H}), 0.88(\mathrm{t}, J=6.8,3 \mathrm{H}) ;{ }^{13} \mathbf{C}$ NMR $\left(\mathrm{CDCl}_{3}, 100 \mathrm{MHz}\right) \delta 156.6,138.7,129.2,128.9,128.6,128.4,127.9,127.0,120.4,110.2$, 84.9, 79.6, 74.3, 55.4, 45.0, 31.1, 22.0, 18.7, 13.8; HRMS: $\mathrm{m} / z\left(\mathrm{M}^{+}\right)$calcd for $\mathrm{C}_{21} \mathrm{H}_{23} \mathrm{O}: 290.1671$ $\left(\mathrm{M}-\mathrm{H}_{2} \mathrm{O}\right)^{+}$, found: 290.1669 .<smiles>CCCCCCC#CC(O)C(c1ccccc1)c1ccccc1OC</smiles>

syn-1-(2-Methoxyphenyl)-2-phenyl-3-decyn-1-ol (syn-5aic)

Purified by silica gel column chromatography $\left(84.1 \mathrm{mg}, 50 \%\right.$ yield, $\mathrm{R}_{f} 0.40$, EtOAc/hexane $\left.=1 / 4\right)$. Colorless oil, ${ }^{1} \mathbf{H}$ NMR $\left(\mathrm{CDCl}_{3}, 400 \mathrm{MHz}\right) \delta 7.24-7.18(\mathrm{~m}, 6 \mathrm{H}), 7.11(\mathrm{dd}, J=2.0,7.2 \mathrm{~Hz}, 1 \mathrm{H})$, 6.87-6.81 (m, 2H), $5.14(\mathrm{t}, J=6.4 \mathrm{~Hz}, 1 \mathrm{H}), 4.13(\mathrm{dt}, J=6.4,2.4 \mathrm{~Hz}, 1 \mathrm{H}), 3.76(\mathrm{~s}, 3 \mathrm{H}), 2.82(\mathrm{~d}, J$ $=6.4 \mathrm{~Hz}, 1 \mathrm{H}), 2.17(\mathrm{dt}, J=2.4,6.8 \mathrm{~Hz}, 2 \mathrm{H}), 1.46-1.41(\mathrm{~m}, 2 \mathrm{H}), 1.35-1.23(\mathrm{~m}, 6 \mathrm{H}), 0.88(\mathrm{t}, J=$ $6.8,3 \mathrm{H}) ;{ }^{13} \mathbf{C}$ NMR $\left(\mathrm{CDCl}_{3}, 100 \mathrm{MHz}\right) \delta 156.6,138.6,129.2,129.1,128.6,128.4,127.9,127.0$, $120.4,110.3,85.0,79.6,74.3,55.4,45.0,31.5,29.0,28.6,22.7,19.0,14.2$; HRMS: $m / z\left(\mathrm{M}^{+}\right)$ calcd for $\mathrm{C}_{23} \mathrm{H}_{27} \mathrm{O}: 318.1984\left(\mathrm{M}-\mathrm{H}_{2} \mathrm{O}\right)^{+}$, found: 318.1980 .<smiles>COCCCC#CC(c1ccccc1)C(O)c1ccccc1</smiles>

syn-1-(2-Methoxyphenyl)-2-phenyl-7-(tert-buthyldimethylsilyl)-3-heptyn-1-ol (syn-5aid) Purified by silica gel column chromatography $\left(116.8 \mathrm{mg}, 55 \%\right.$ yield, $\mathrm{R}_{f} 0.35$, EtOAc/hexane $=$ 1/4). Colorless oil, ${ }^{1} \mathbf{H}$ NMR $\left(\mathrm{CDCl}_{3}, 400 \mathrm{MHz}\right) \delta 7.24-7.18(\mathrm{~m}, 6 \mathrm{H}), 7.10(\mathrm{dd}, J=1.6,7.6 \mathrm{~Hz}$, $1 \mathrm{H}), 6.87-6.81(\mathrm{~m}, 2 \mathrm{H}), 5.14(\mathrm{t}, J=6.4 \mathrm{~Hz}, 1 \mathrm{H}), 4.11(\mathrm{dt}, J=6.4,2.0 \mathrm{~Hz}, 1 \mathrm{H}), 3.77(\mathrm{~s}, 3 \mathrm{H}), 3.60$ $(\mathrm{dt}, J=3.2,6.4 \mathrm{~Hz}, 2 \mathrm{H}), 2.80(\mathrm{~d}, J=6.4 \mathrm{~Hz}, 1 \mathrm{H}), 2.25(\mathrm{dt}, J=2.0,6.8 \mathrm{~Hz}, 2 \mathrm{H}), 1.68-1.61(\mathrm{~m}$, $2 \mathrm{H}), 0.88(\mathrm{~s}, 9 \mathrm{H}), 0.03(\mathrm{~s}, 6 \mathrm{H}) ;{ }^{13} \mathbf{C}$ NMR $\left(\mathrm{CDCl}_{3}, 100 \mathrm{MHz}\right) \delta 156.6,138.5, \mathrm{~s} 129.2,129.0,128.6$, 128.3, 127.9, 127.0, 120.3, 110.2, 84.3, 79.8, 74.3, 61.8, 55.3, 45.0, 32.0, 26.1, 18.4, 15.4, -5.2; HRMS: $m / z\left(\mathrm{FAB}^{+}\right)$calcd for $\mathrm{C}_{26} \mathrm{H}_{37} \mathrm{O}_{3} \mathrm{Si}: 425.2512(\mathrm{M}+\mathrm{H})$, found: 425.2500 . 
<smiles>CCCCOCCCCC#CC(O)C(O)c1ccccc1</smiles>

syn-1-(2-Methoxyphenyl)-2-phenyl-7-(tert-buthyldimethylsilyl)-3-octyn-1-ol (syn-5aie)

Purified by silica gel column chromatography $\left(131.6 \mathrm{mg}, 60 \%\right.$ yield, $\mathrm{R}_{f} 0.40$, EtOAc/hexane $=$ 1/4). Colorless oil, ${ }^{1} \mathbf{H}$ NMR $\left(\mathrm{CDCl}_{3}, 400 \mathrm{MHz}\right) \delta 7.24-7.18(\mathrm{~m}, 6 \mathrm{H}), 7.10(\mathrm{dd}, J=2.0,7.2 \mathrm{~Hz}$, $1 \mathrm{H}), 6.87-6.81(\mathrm{~m}, 2 \mathrm{H}), 5.13(\mathrm{t}, J=6.4 \mathrm{~Hz}, 1 \mathrm{H}), 4.12(\mathrm{dt}, J=6.4,2.4 \mathrm{~Hz}, 1 \mathrm{H}), 3.76(\mathrm{~s}, 3 \mathrm{H}), 3.58$ (t, $J=6.0 \mathrm{~Hz}, 2 \mathrm{H}), 2.82(\mathrm{~d}, J=6.4 \mathrm{~Hz}, \mathrm{OH}), 2.19(\mathrm{dt}, J=2.4,6.4 \mathrm{~Hz}, 2 \mathrm{H}), 1.53-1.50(\mathrm{~m}, 4 \mathrm{H})$, 0.89 (s, 9H), $0.04(\mathrm{~s}, 6 \mathrm{H}) ;{ }^{13} \mathbf{C}$ NMR $\left(\mathrm{CDCl}_{3}, 100 \mathrm{MHz}\right) \delta 156.6,138.6,129.3,129.1,128.6,128.4$, 128.0, 127.0, 120.4, 110.3, 84.7, 79.9, 74.3, 62.8, 55.4, 45.0, 32.0, 26.1, 25.5, 18.9, 18.5, -5.2; HRMS: $m / z\left(\mathrm{FAB}^{+}\right)$calcd for $\mathrm{C}_{27} \mathrm{H}_{38} \mathrm{O}_{3} \mathrm{Si}: 439.2668(\mathrm{M}+\mathrm{H})$, found: 439.2655 .

\section{General Procedure for Scheme 5b}

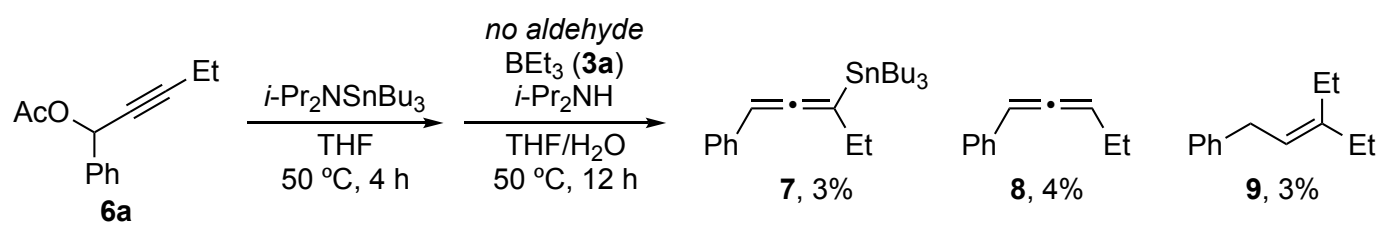

Diisopropylamino)tributylstannane $(0.5 \mathrm{M}$ in THF, $2.4 \mathrm{~mL}, 1.2 \mathrm{mmol})$ was added to a flask charged with $6 \mathbf{6}(174.2 \mathrm{mg}, 1.0 \mathrm{mmol})$ at room temperature under an argon atmosphere, and then it was heated to $50{ }^{\circ} \mathrm{C}$ in an oil bath for 4 hours. After the reaction completed checked with TLC (S.M.: $\mathrm{R}_{f}=0.45,1 \mathrm{a}: \mathrm{R}_{f}=0.61$, EtOAc/hexane $\left.=1 / 5\right)$, diisopropylamine $(168 \mu \mathrm{L}, 1.2 \mathrm{mmol})$, triethylborane (1 M in hexane, $1.2 \mathrm{~mL}, 1.2 \mathrm{mmol})$, and $\mathrm{H}_{2} \mathrm{O}(0.6 \mathrm{~mL})$ were successively added to the mixture at room temperature. The reaction mixture was stirred $50{ }^{\circ} \mathrm{C}$ for 30 minutes. Upon completion of the reaction, the reaction mixture was diluted with $\mathrm{Et}_{2} \mathrm{O}(15 \mathrm{~mL})$ and washed with saturated $\mathrm{NH}_{4} \mathrm{Cl}(2 \times 10 \mathrm{~mL})$, saturated $\mathrm{NaHCO}_{3}(2 \times 10 \mathrm{~mL})$, and brine $(2 \times 10 \mathrm{~mL})$. The combined organic layers were dried over $\mathrm{MgSO}_{4}$ and concentrated. The residue was purified by silica gel chromatography $\left(10 \% \mathrm{w} / \mathrm{w} \mathrm{K} \mathrm{CO}_{3}\right.$-silica gel, $\mathrm{R}_{f} 0.43$, hexane). Compounds $7^{[14]}$ (cas: 2270914-96-8), $\mathbf{8}^{[19]}$ (cas: 2327-97-1), and $\mathbf{9}^{[14][20]}$ (cas: 134165-97-2) were isolated as an inseparable mixture (colorless oil, $21.4 \mathrm{mg}$ ).

\section{1-Phenyl-3-tributylstannyl-penta-1,2-diene (7) ${ }^{[14]}$}

Characteristic NMR data: ${ }^{1} \mathbf{H}$ NMR $\left(\mathrm{CDCl}_{3}, 400 \mathrm{MHz}\right) \delta 5.72(\mathrm{t}, J=3.2 \mathrm{~Hz}, 1 \mathrm{H}), 2.24(\mathrm{q}, J=7.2$ $\mathrm{Hz}, 1 \mathrm{H}), 2.23$ (q, $J=7.2 \mathrm{~Hz}, 1 \mathrm{H}), 1.56-1.48(\mathrm{~m}, 6 \mathrm{H}), 1.29$ (sext, $J=7.2 \mathrm{~Hz}, 6 \mathrm{H}), 0.87$ (t, $J=7.2$ $\mathrm{Hz}, 9 \mathrm{H}) ;{ }^{13} \mathbf{C} \mathbf{N M R}\left(\mathrm{CDCl}_{3}, 100 \mathrm{MHz}\right) \delta 200.1,137.7,99.3,87.0$.

\section{1-Phenylpenta-1,2-diene (8) ${ }^{[19]}$}

Characteristic ${ }^{1} \mathrm{H}$ NMR data: ${ }^{1} \mathbf{H}$ NMR $\left(\mathrm{CDCl}_{3}, 400 \mathrm{MHz}\right) \delta 6.15(\mathrm{dt}, J=6.4,4.0 \mathrm{~Hz}, 1 \mathrm{H}), 5.63$ (q, $J=6.4 \mathrm{~Hz}, 1 \mathrm{H}) ;{ }^{13} \mathbf{C ~ N M R}\left(\mathrm{CDCl}_{3}, 100 \mathrm{MHz}\right) \delta 205.0,135.3,96.9,95.3,22.1$.

\section{1-Phenyl-3-ethylpent-2-ene (9) ${ }^{[14][20]}$}

Characteristic ${ }^{1} \mathrm{H}$ NMR data: ${ }^{1} \mathbf{H}$ NMR $\left(\mathrm{CDCl}_{3}, 400 \mathrm{MHz}\right) \delta 5.28(\mathrm{t}, J=7.2 \mathrm{~Hz}, 1 \mathrm{H}), 3.38(\mathrm{~d}, J=$ $7.2 \mathrm{~Hz}, 1 \mathrm{H}), 2.07(\mathrm{q}, J=7.2 \mathrm{~Hz}, 2 \mathrm{H}) ;{ }^{13} \mathbf{C} \mathbf{~ N M R}\left(\mathrm{CDCl}_{3}, 100 \mathrm{MHz}\right) \delta 144.0,142.1,121.2,33.9$. 


\section{Assignment of relative configuration}
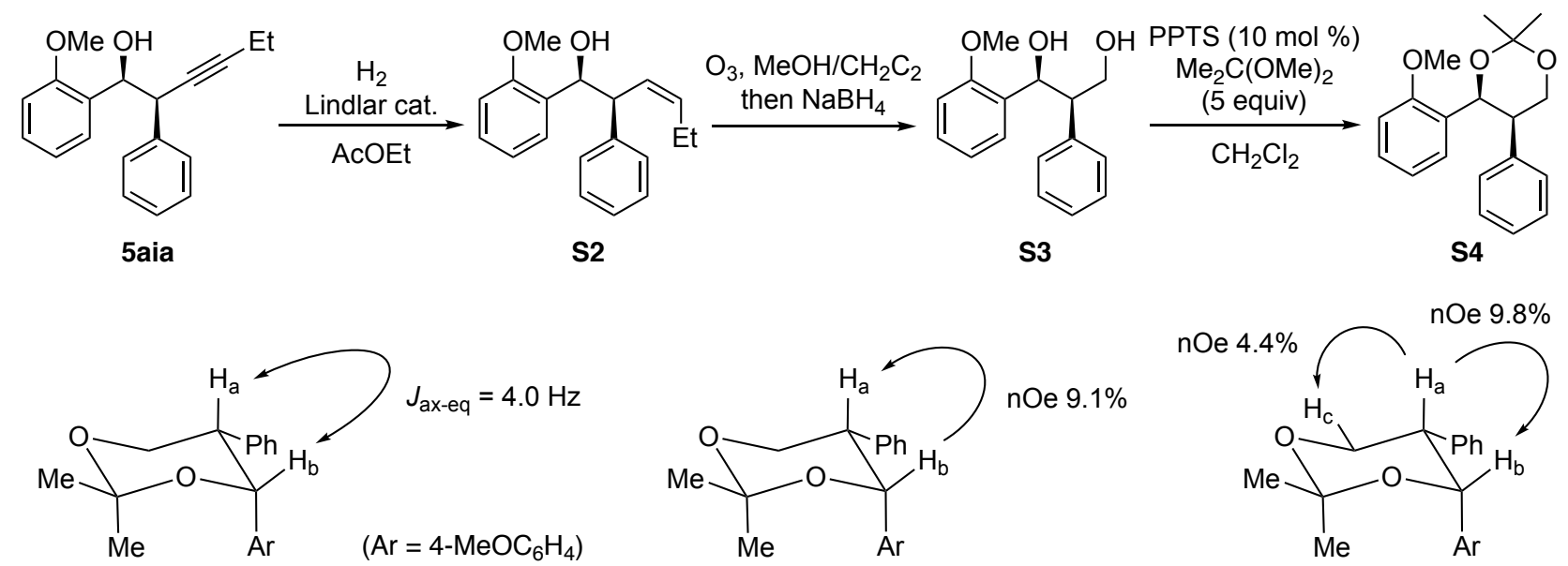

syn-(Z)-1-(2-Methoxyphenyl)-2-phenyl-hex-3-en-1-ol (S2).

To a stirred solution of syn-1-(2-methoxyphenyl)-2-phenyl-hex-3-yn-1-ol (5aia) (280.3 mg, 1.0 mmol) in EtOAc $(10 \mathrm{~mL})$ was added Lindlar catalyst $(41.4 \mathrm{mg})$. The atmosphere in the flask was replaced to hydrogen under balloon pressure, and the reaction mixture was vigorously stirred at room temperature for 3 hours. Upon completion, the mixture was filtered through a pad of Celite to afford crude material, which was purified by silica gel chromatography to give $\mathbf{S 2}$ as a colorless sticky oil (386.8 mg, 100\%, $\mathrm{R}_{f} 0.43$, EtOAc/hexane $\left.=3 / 7\right)$.

${ }^{1} \mathbf{H}$ NMR $\left(\mathrm{CDCl}_{3}, 400 \mathrm{MHz}\right) \delta 7.32-7.19(\mathrm{~m}, 7 \mathrm{H}), 6.90(\mathrm{dt}, J=1.2,7.6 \mathrm{~Hz}, 1 \mathrm{H}), 6.84(\mathrm{dm}, J=8.0$ $\mathrm{Hz}, 1 \mathrm{H}), 5.62$ (tt, $J=1.6,10.4 \mathrm{~Hz}, 1 \mathrm{H}), 5.25$ (ddt, $J=0.8,7.4,10.8 \mathrm{~Hz}, 1 \mathrm{H}), 5.14(\mathrm{dd}, J=6.8,8.0$ $\mathrm{Hz}, 1 \mathrm{H}), 4.03$ (t, $J=9.2 \mathrm{~Hz}, 1 \mathrm{H}), 3.81(\mathrm{~s}, 3 \mathrm{H}), 2.47(\mathrm{~d}, J=6.4 \mathrm{~Hz}, 1 \mathrm{H}), 1.93-1.76(\mathrm{~m}, 2 \mathrm{H}), 0.68(\mathrm{t}$, $J=7.6 \mathrm{~Hz}, 3 \mathrm{H}) ;{ }^{13} \mathbf{C} \mathbf{N M R}\left(\mathrm{CDCl}_{3}, 100 \mathrm{MHz}\right) \delta 156.8,142.3,132.5,130.6,129.0,128.5,128.45$, $128.36,128.1,126.5,120.5,110.4,74.1,55.3,51.0,20.7,13.8$.

\section{1-(2-Methoxyphenyl)-2-phenylpropane-1,3-diol (S3).}

S3 $(285.6 \mathrm{mg}, 1.0 \mathrm{mmol})$ was dissolved in dry dichloromethane $(22 \mathrm{ml})$ and methanol $(22 \mathrm{~mL})$ and cooled down to $-78{ }^{\circ} \mathrm{C}$. A stream of ozone was bubbled through until solution turned blue, then $\mathrm{N}_{2}$ was bubbled through until all the blue color disappeared. Sodium borohydride (190.1 mg, $5.1 \mathrm{mmol}$ ) was added in four equal portions every 15 minutes for the duration of one hour, while maintaining the temperature at $-78{ }^{\circ} \mathrm{C}$. The reaction was left to stir at room temperature until complete by TLC analysis. The solvent was removed under reduced pressure. The residue was retaken in chloroform $(25 \mathrm{~mL})$ and washed with brine. The aqueous wash was extracted with chloroform $(3 \times 25 \mathrm{~mL})$, and the combined organic layers were dried with $\mathrm{MgSO}_{4}$, filtered, and the solvent was removed under reduced pressure to give an oil, which was purified using column chromatography (20 to $40 \%$ EtOAc/Hexane) to give the diol as a colorless oil $\left(217.3 \mathrm{mg}, 84 \%, \mathrm{R}_{f}\right.$ 0.44 , EtOAc/hexane $=1 / 1)$.

${ }^{1} \mathbf{H}$ NMR $\left(\mathrm{CDCl}_{3}, 400 \mathrm{MHz}\right) \delta 7.34-7.30(\mathrm{~m}, 2 \mathrm{H}), 7.28-7.22(\mathrm{~m}, 5 \mathrm{H}), 6.94(\mathrm{dt}, J=1.2,7.6 \mathrm{~Hz}$, $1 \mathrm{H}), 6.87(\mathrm{dd}, J=1.2,8.8 \mathrm{~Hz}, 1 \mathrm{H}), 5.35(\mathrm{~d}, J=7.6 \mathrm{~Hz}, 1 \mathrm{H}), 3.84-3.75(\mathrm{~m}, 2 \mathrm{H}), 3.77(\mathrm{~s}, 3 \mathrm{H}), 3.16$ $(\mathrm{dt}, J=7.6,5.6 \mathrm{~Hz}, 1 \mathrm{H}), 2.52(\mathrm{br} \mathrm{s}, 1 \mathrm{H}) ;{ }^{13} \mathbf{C ~ N M R}\left(\mathrm{CDCl}_{3}, 100 \mathrm{MHz}\right) \delta 156.3,139.3,130.4$, 129.2, 128.4, 128.3, 127.7, 127.0, 120.9, 110.4, 70.5, 64.3, 55.3, 54.8; HRMS: $m / z\left(\mathrm{M}^{+}\right)$calcd for $\mathrm{C}_{16} \mathrm{H}_{18} \mathrm{O}_{3}: 258.1256$, found: 258.1256 . 


\section{2,2-Dimethyl-4-(2-methoxyphenyl)-5-phneyl-1,3-dioxane (S4).}

To a solution of $\mathbf{S 4}(191.8 \mathrm{mg}, 0.74 \mathrm{mmol})$ in dry dichloromethane $(6 \mathrm{~mL})$ were added 2,2dimethoxypropane $(450 \mu \mathrm{L}, 3.7 \mathrm{mmol})$ and PPTS $(18.6 \mathrm{mg}, 74 \mu \mathrm{mol})$ at $0{ }^{\circ} \mathrm{C}$, and the mixture was stirred at room temperature for 2 hours. To the mixture was added saturated $\mathrm{NaHCO}_{3}$ aqueous solution at $0{ }^{\circ} \mathrm{C}$, and the aqueous layer was extracted with ethyl acetate $(2 \times 20 \mathrm{~mL})$. The organic layer was washed with brine $(2 \times 20 \mathrm{~mL})$. The combined organic layers were dried over $\mathrm{MgSO}_{4}$ and concentrated. The residue was purified by silica gel chromatography to give $\mathbf{S} \mathbf{4}$ as a colorless solid (200.1 mg, 91\%, R 0.49 , EtOAc/hexane = 1/4).

${ }^{1} \mathbf{H}$ NMR $\left(\mathrm{CDCl}_{3}, 400 \mathrm{MHz}\right) \delta$ 7.23-7.21 (m, 4H), 7.07-7.02 (m, 4H), $6.89(\mathrm{dd}, J=1.2,7.6 \mathrm{~Hz}$, $1 \mathrm{H}), 6.75(\mathrm{~d}, J=7.6 \mathrm{~Hz}, 1 \mathrm{H}), 6.62(\mathrm{dt}, J=0.8,7.2 \mathrm{~Hz}, 1 \mathrm{H}), 5.67(\mathrm{~d}, J=3.6 \mathrm{~Hz}, 1 \mathrm{H}), 4.57(\mathrm{dd}, J=$ $4.0,11.6 \mathrm{~Hz}, 1 \mathrm{H}), 4.08(\mathrm{dd}, J=4.0,11.6 \mathrm{~Hz}, 1 \mathrm{H}), 3.87(\mathrm{~s}, 3 \mathrm{H}), 3.08(\mathrm{dt}, J=1.6,3.6 \mathrm{~Hz}, 1 \mathrm{H}), 1.67$ $(\mathrm{s}, 3 \mathrm{H}), 1.65(\mathrm{~s}, 3 \mathrm{H}) ;{ }^{13} \mathbf{C}$ NMR $\left(\mathrm{CDCl}_{3}, 100 \mathrm{MHz}\right) \delta 155.2,140.8,129.5,128.7,127.5,127.4$, 127.2, 126.1, 120.2, 109.3, 99.4, 68.5, 65.0, 55.2, 42.7, 29.7, 19.3; HRMS: $\mathrm{m} / z\left(\mathrm{M}^{+}\right)$calcd for $\mathrm{C}_{19} \mathrm{H}_{22} \mathrm{O}_{3}: 298.1569$, found: 298.1569 .

The indicated coupling constant is consistent with similar compounds reported previously. ${ }^{[21]}$

\section{Preparation of $(R)-6 a$}

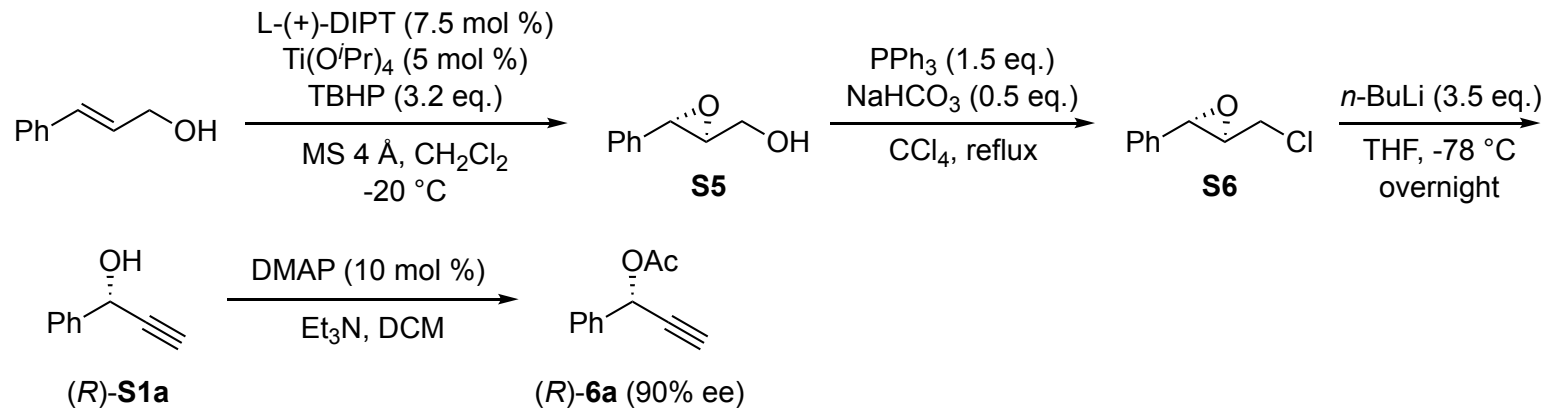

\section{(2S,3S)-3-Phenyloxiran-2-yl)methanol (S5, CAS: 125411-08-7)}

To a $200 \mathrm{~mL}$ two neck round-bottom flask charged with activated molecular sieves $4 \AA$ (1.4 g), L(+)-diisopropyl tartrate $(347.0 \mathrm{mg}, 1.48 \mathrm{mmol})$ and $\mathrm{CH}_{2} \mathrm{Cl}_{2}(40 \mathrm{~mL})$ were added and the mixture was cooled to $-20^{\circ} \mathrm{C}$. Next, Ti $\left(\mathrm{O}-{ }^{i} \mathrm{Pr}\right)_{4}$ and TBHP $(1.8 \mathrm{M}$ solution in toluene, $40 \mathrm{~mL})$ were added. After the mixture was stirred at $-20^{\circ} \mathrm{C}$ for 30 minutes, a solution of cinnamyl alcohol $(2.65 \mathrm{~g}, 19.7$ mmol) in $8 \mathrm{~mL}$ of $\mathrm{CH}_{2} \mathrm{Cl}_{2}$ were added dropwise over 15 minutes and the reaction mixture was stirred overnight. The solution was quenched with $2 \mathrm{~mL}$ of a $10 \% \mathrm{NaOH}$ aqueous solution saturated with sodium chloride and diethyl ether $(10 \mathrm{~mL})$ at $0{ }^{\circ} \mathrm{C}$. And $\mathrm{MgSO}_{4}(2 \mathrm{~g})$ and Celite ${ }^{\circledR}$ $(0.2 \mathrm{~g})$ were added. After 15 minutes of stirring, the mixture was filtered through a pad of Celite ${ }^{\circledR}$. The solvent was removed under reduced pressure. The residue was purified by silica gel chromatography to give the product S5 $(2.5 \mathrm{~g}, 84 \%)$. The spectral data of product are consistent with those of the literature. ${ }^{[22][23]}$

(2R,3S)-2-(Chloromethyl)-3-phenyloxirane (S6, CAS: 125411-08-7) ${ }^{[24]}$

A stirred solution of $\mathbf{S 5}(2.5 \mathrm{~g}, 16.6 \mathrm{mmol})$, triphenyl phosphine $(7.4 \mathrm{~g}, 28.2 \mathrm{mmol})$ and $\mathrm{NaHCO}_{3}$ $(800 \mathrm{mg}, 9.5 \mathrm{mmol})$ in $\mathrm{CCl}_{4}(36 \mathrm{~mL})$ under argon atmosphere was refluxed in an oil bath for 3 hours. The solution was filtered through a pad of Celite ${ }^{\circledR}$. The solvent was removed in vacuo and the residue was purified by silica gel chromatography to give product $\mathbf{S 6}(2.0 \mathrm{~g}, 72 \%)$. ${ }^{[25]}$

A procedure reported by Ogasawara was used to prepare (R)-S1a (CAS: 61317-73-5) 
To a solution of $\mathbf{S 6}(2.0 \mathrm{~g}, 11.9 \mathrm{mmol})$ in THF $(20 \mathrm{~mL})$ was added $n$-BuLi $(36.6 \mathrm{~mL}, 1.6 \mathrm{M}$ in hexane, $41.7 \mathrm{mmol}$ ) dropwise over 15 minutes at $-78^{\circ} \mathrm{C}$. After 2 hours, the resulting solution was quenched by addition of saturated $\mathrm{NH}_{4} \mathrm{Cl}$. The organic phase was separated and the aqueous phase was extracted with $\mathrm{Et}_{2} \mathrm{O}$. The combined organic layers were dried over $\mathrm{MgSO}_{4}$ and concentrated in vacuo. The residue was purified by silica gel chromatography to give $(R)$-S1a $(1.1 \mathrm{~g}, 69 \%)$.

\section{(R)-1-Acetoxy-1-phenyl-2-propyne ( $R$ )-6a, CAS: 84681-19-6)}

To a solution of $(R)-S 1 a(132.2 \mathrm{mg}, 1.0 \mathrm{mmol})$, N,N-dimethyl-4-aminopyridine $(14.3 \mathrm{mg}, 0.1$ mmol) and $\mathrm{Et}_{3} \mathrm{~N}(279 \mu \mathrm{L}, 2 \mathrm{mmol})$ in $\mathrm{CH}_{2} \mathrm{Cl}_{2}(5 \mathrm{~mL})$ was added acetic acid anhydride $(142 \mu \mathrm{L}$, $1.5 \mathrm{mmol}$ ) at $0{ }^{\circ} \mathrm{C}$ under a argon atmosphere. After stirring at room temperature for 2 hours, the reaction mixture was quenched with saturated aqueous $\mathrm{NH}_{4} \mathrm{Cl}$. The aqueous phase was extracted with $\mathrm{CH}_{2} \mathrm{Cl}_{2}$, and the combined organic extracts were washed with aqueous $\mathrm{NaHCO}_{3}$ and then brine. After the organic layer was dried over $\mathrm{MgSO}_{4}$, the solvent was removed under reduced pressure. The residue was purified by silica gel chromatography $\left(\mathrm{R}_{f} 0.52\right.$, EtOAc/hexane $\left.=3 / 7\right)$ to give $(R)-6 a$ as a yellow oil (158.5 $\mathrm{mg}, 91 \%)$.

Spectral data is in accordance with the literature. ${ }^{[5]}$

Determination of enantiomeric excess of $(R)-\mathbf{6 a}(90 \%$ ee)

HPLC: Chiralcel OZ-H, $n$-Hexane: $i$-PrOH $=97: 3$, flow: $0.5 \mathrm{~mL} / \mathrm{min}, \lambda=217 \mathrm{~nm}$ 
Racemic sample of $\mathbf{6 a}$

HPLC: CHIRALCEL OZ-H, $n$-Hexane: $i-\mathrm{PrOH}=97: 3$, flow: $0.5 \mathrm{~mL} / \mathrm{min}, \lambda=217 \mathrm{~nm}$

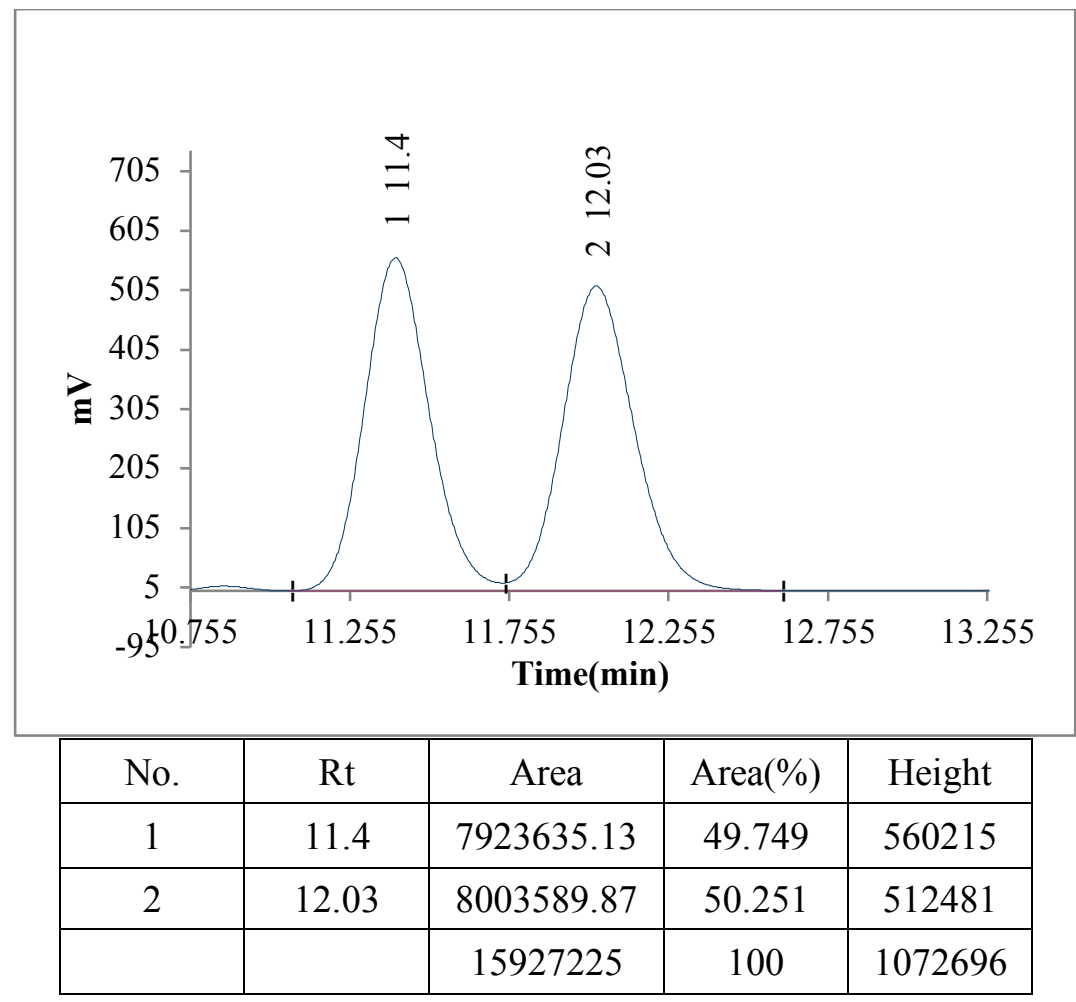

$(R)-\mathbf{6 a}$

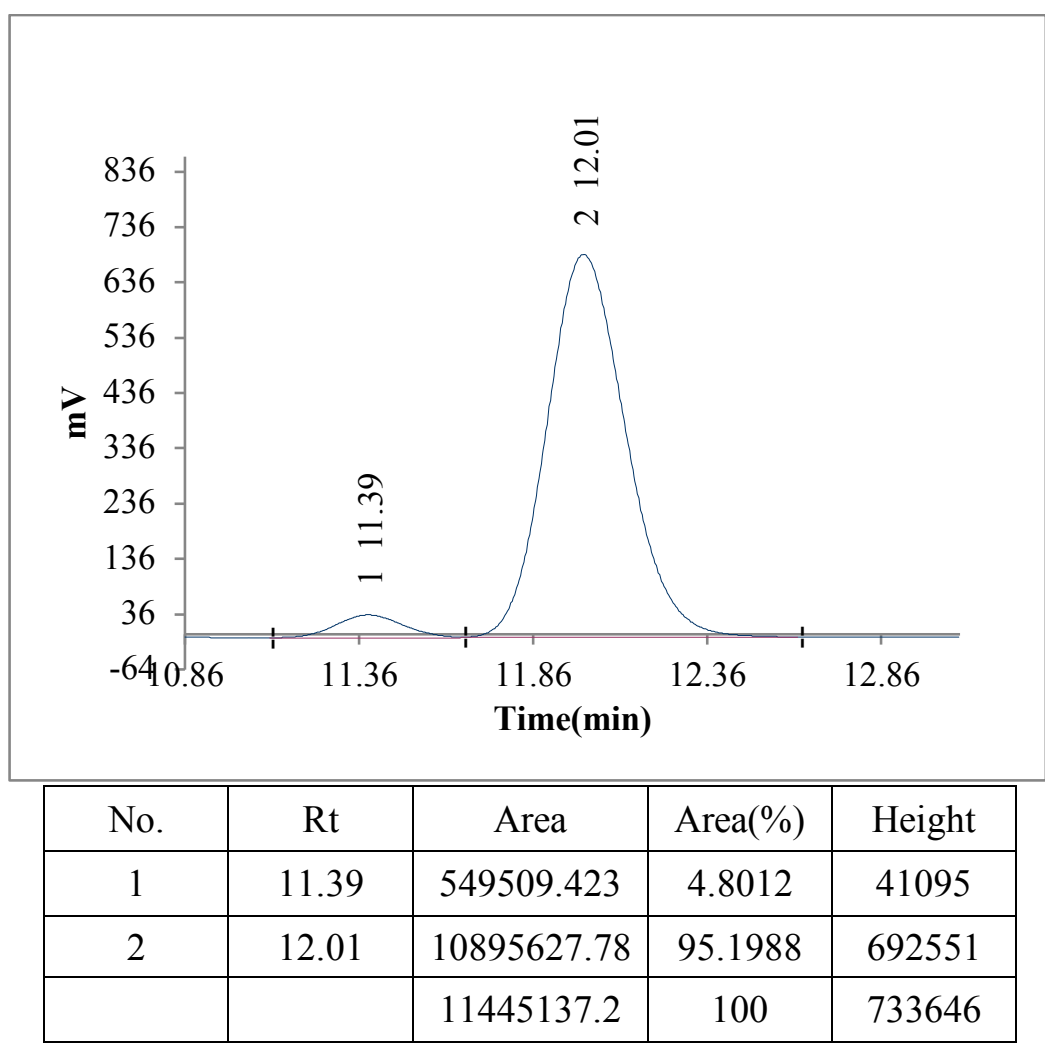




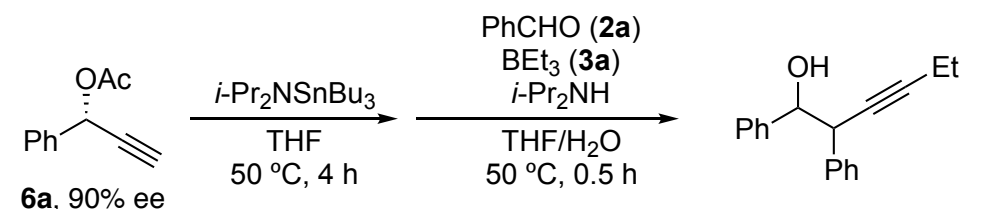

6a, $90 \%$ ee

5aaa, $70 \%$, syn/anti $=2.5: 1$ syn-5aaa, $83 \%$ ee, $92 \%$ es anti-5aaa, $84 \%$ ee, $93 \%$ es

Racemic sample of syn-5aaa

HPLC: CHIRALCEL IA, $n$-Hexane: $i-\mathrm{PrOH}=96: 4$, flow: $1.0 \mathrm{~mL} / \mathrm{min}, \lambda=210 \mathrm{~nm}$

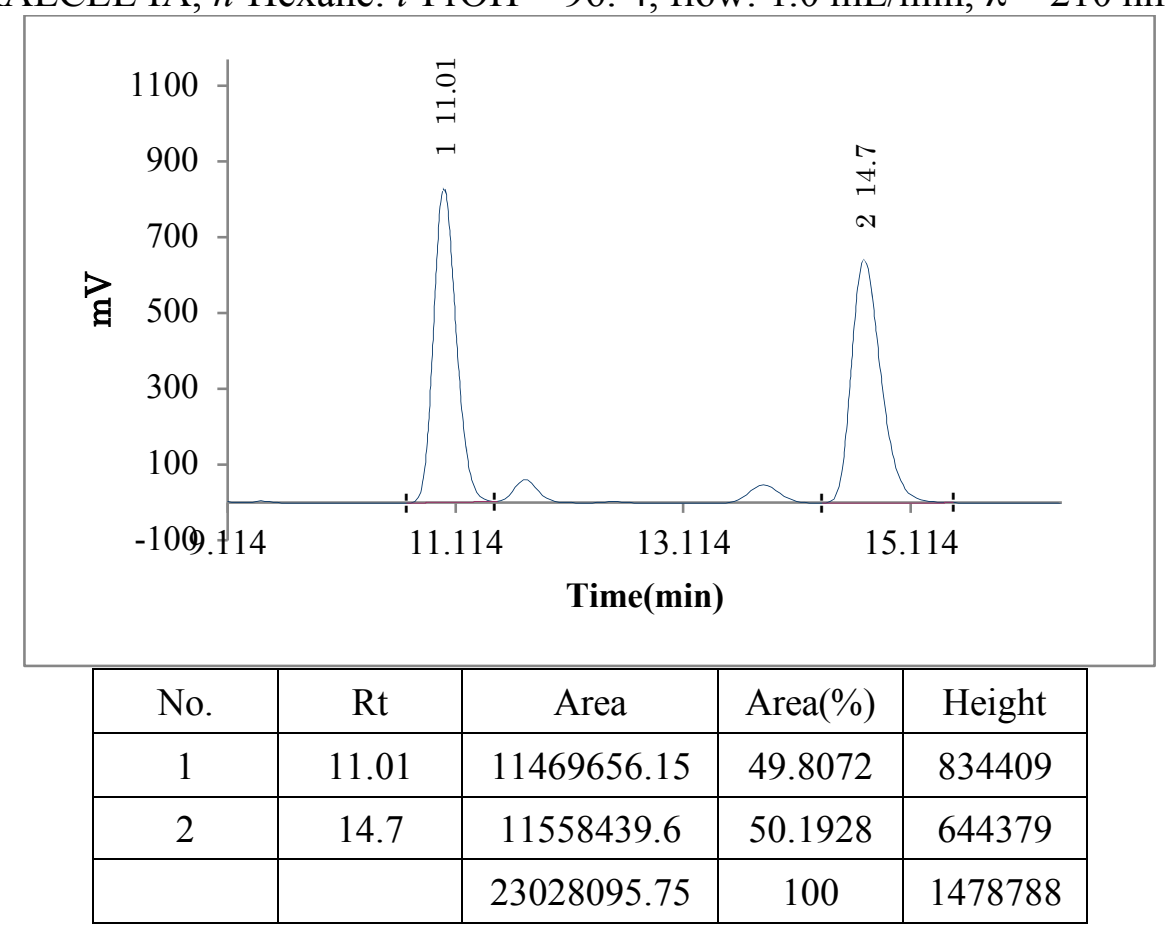

Chiral sample of syn-5aaa

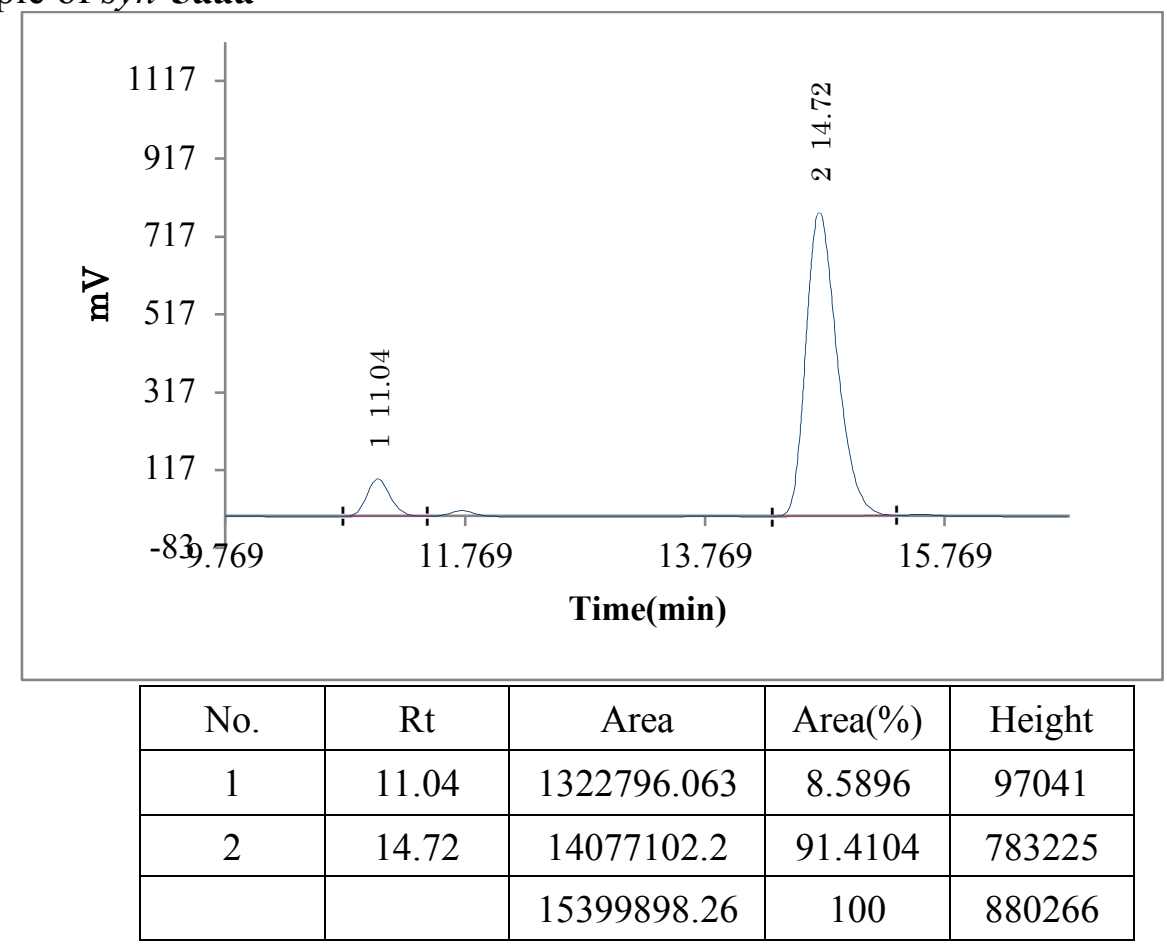


Racemic sample of anti-5aaa

HPLC: CHIRALCEL IA, $n$-Hexane: $i-\mathrm{PrOH}=96: 4$, flow: $1.0 \mathrm{~mL} / \mathrm{min}, \lambda=210 \mathrm{~nm}$

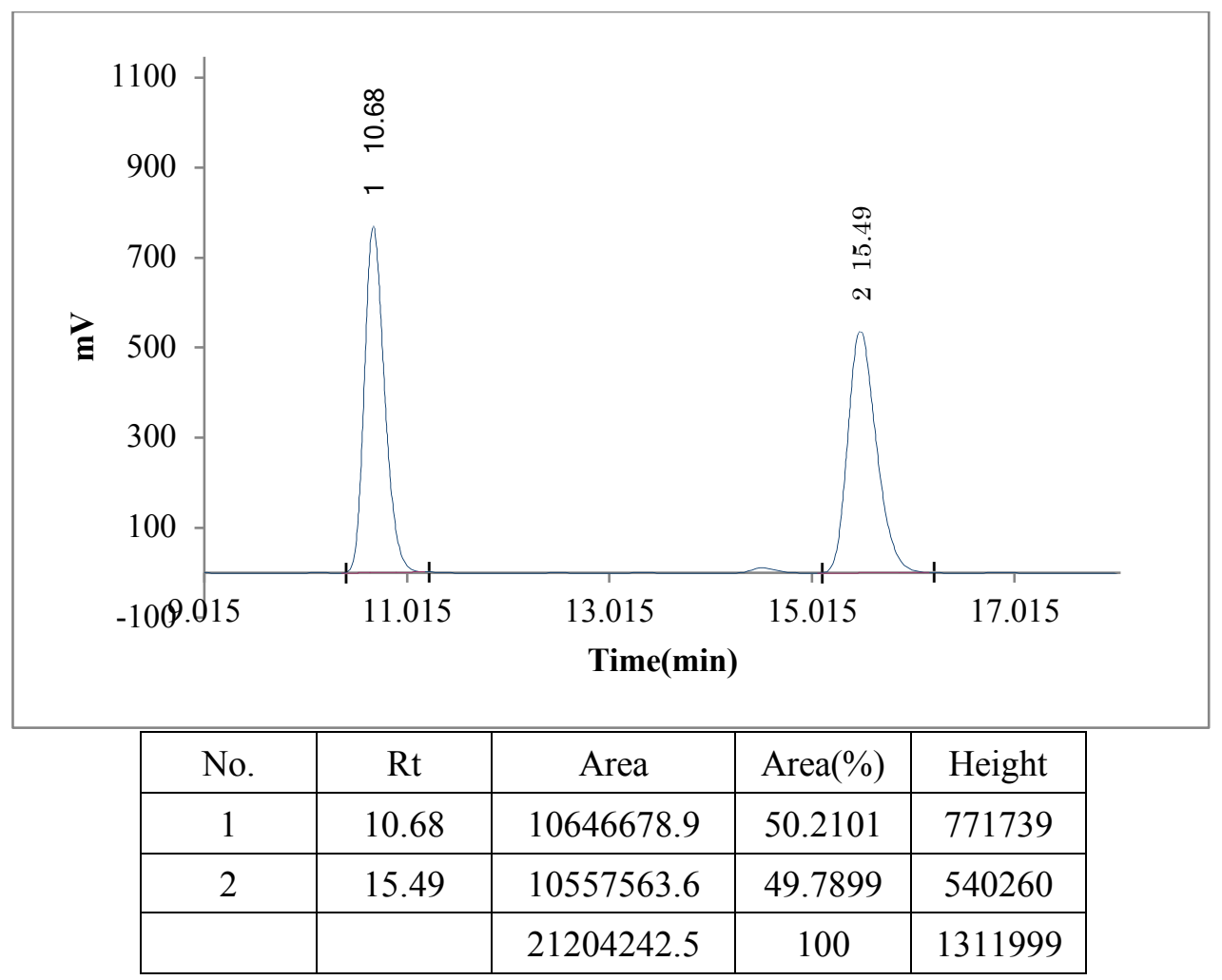

Chiral sample of anti-5aaa

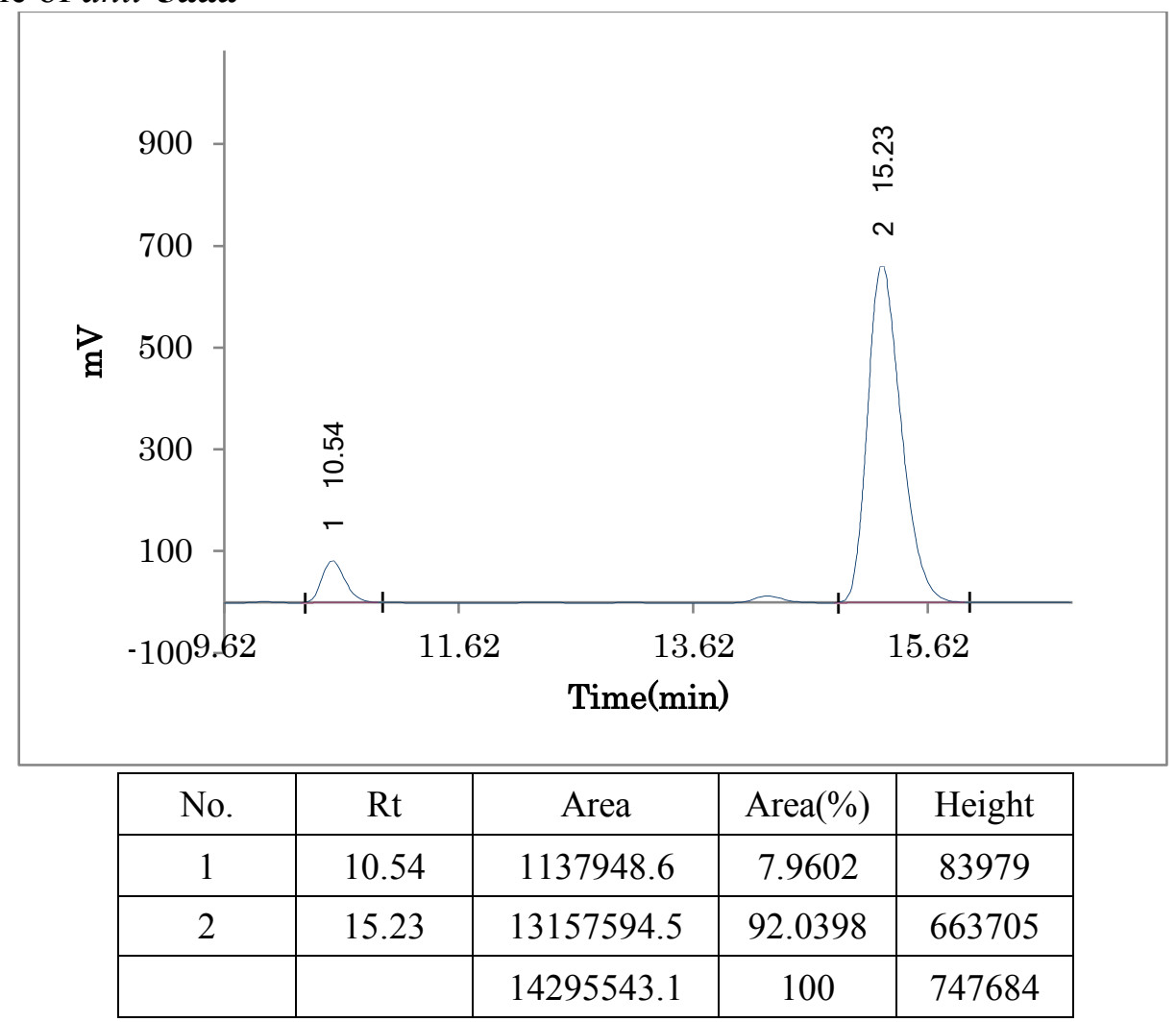




\section{Determination of Absolute Stereochemistry of syn-5aaa}

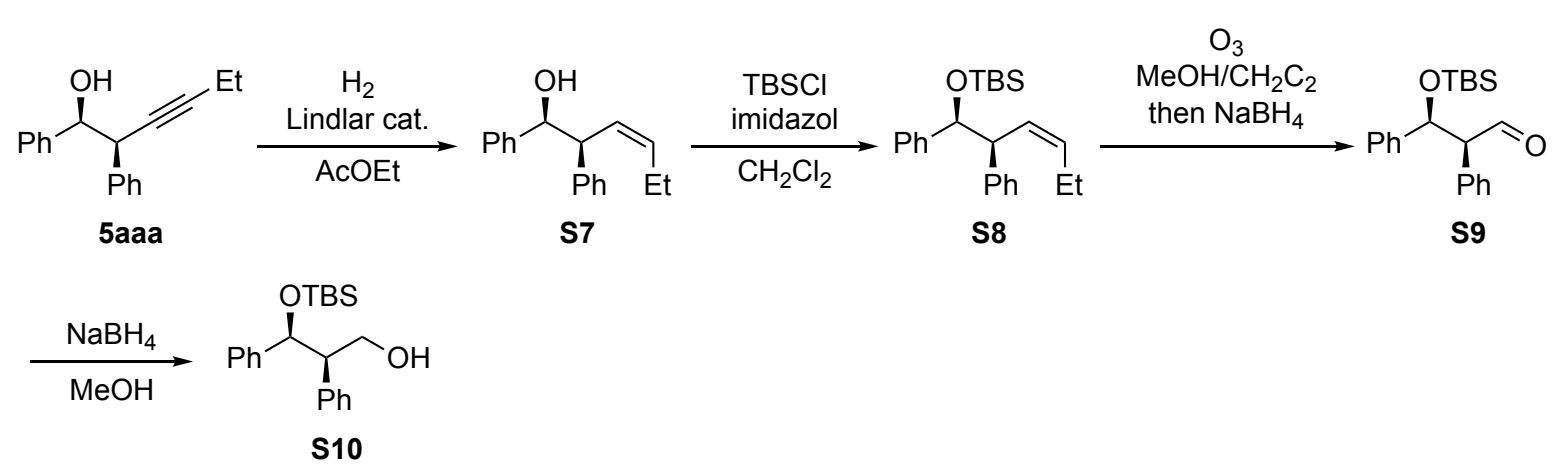

To a stirred solution of syn-1,2-diphenylhex-3-yn-1-ol (5aaa) $(277.5 \mathrm{mg}, 1.1 \mathrm{mmol})$ in EtOAc (11 $\mathrm{mL}$ ) was added Lindlar catalyst $(48.7 \mathrm{mg})$. The atmosphere in the flask was replaced to hydrogen under balloon pressure, and the reaction mixture was vigorously stirred at room temperature for 1 hour. Upon completion, the mixture was filtered through a pad of Celite ${ }^{\circledR}$ to afford $\mathbf{S} 7$ as a crude material, which was directly used for next step without further purification.

${ }^{1} \mathbf{H}$ NMR $\left(\mathrm{CDCl}_{3}, 400 \mathrm{MHz}\right) \delta 7.37-7.22(\mathrm{~m}, 10 \mathrm{H}), 5.53(\mathrm{ddt}, J=9.2,10.8,1.6 \mathrm{~Hz}, 1 \mathrm{H}), 5.28$ (ddt, $J=0.8,7.2,10.8 \mathrm{~Hz}, 1 \mathrm{H}), 4.81(\mathrm{~d}, J=8.4 \mathrm{~Hz}, 1 \mathrm{H}), 3.88(\mathrm{dd}, J=8.4,9.2 \mathrm{~Hz}, 1 \mathrm{H}), 1.94$ (br s, $1 \mathrm{H})$, $1.89-1.67(\mathrm{~m}, 2 \mathrm{H}), 0.63(\mathrm{t}, J=7.6 \mathrm{~Hz}, 3 \mathrm{H}) ;{ }^{13} \mathbf{C ~ N M R}\left(\mathrm{CDCl}_{3}, 100 \mathrm{MHz}\right) \delta 142.2,141.9,133.6$, $128.9,128.5,128.12,128.08,127.8,127.2,127.0,78.3,52.6,20.9,13.7$.

A solution of $\mathbf{S 7}(120.3 \mathrm{mg}, 0.52 \mathrm{mmol})$ and tert-butyldimethylsilyl chloride (116.7 $\mathrm{mg}, 0.78$ mmol, 1.5 equiv) in $\mathrm{CH}_{2} \mathrm{Cl}_{2}(3 \mathrm{~mL})$ was stirred until a homogeneous solution was obtained. Imidazole (70.3 mg, $1.03 \mathrm{mmol}, 2$ equiv) was added in one portion, and the white suspension was stirred for 12 hours at room temperature under an argon atmosphere. Upon completion, water (10 $\mathrm{mL})$ was added to the mixture and the whole was extracted with EtOAc $(10 \mathrm{~mL})$ three times. Combined organic layers were washed with brine $(10 \mathrm{~mL})$ and dried over $\mathrm{MgSO}_{4}$, filtered, and concentrated in vacuo. The residue was purified by silica gel column chromatography $\left(\mathrm{R}_{f} 0.70\right.$, EtOAc/hexane $=4 / 1$, TLC stain: $p$-anisaldehyde) to provide $\mathbf{S 8}$ a colorless oil $(160.5 \mathrm{mg}, 85 \%)$.

${ }^{1} \mathrm{H}$ NMR $\left(\mathrm{CDCl}_{3}, 400 \mathrm{MHz}\right) \delta 7.28-7.14(\mathrm{~m}, 10 \mathrm{H}), 5.60$ (ddt, $\left.J=10.4,2.0 \mathrm{~Hz}, 1 \mathrm{H}\right), 5.25$ (ddt, $J=$ $0.8,10.4,7.2 \mathrm{~Hz}, 1 \mathrm{H}), 4.71(\mathrm{~d}, J=7.6 \mathrm{~Hz}, 1 \mathrm{H}), 3.78$ (dd, $J=7.6,10.4 \mathrm{~Hz}, 1 \mathrm{H}), 1.84$ (dq, $J=2.0$, $7.2 \mathrm{~Hz}, 1 \mathrm{H}), 1.76(\mathrm{dq}, J=2.0,7.2 \mathrm{~Hz}, 1 \mathrm{H}), 0.69$ (s, 9H), 0.65 (t, $J=7.2 \mathrm{~Hz}, 3 \mathrm{H}),-0.29$ (s, 3H), $0.37(\mathrm{~s}, 3 \mathrm{H}) ;{ }^{13} \mathrm{C}$ NMR $\left(\mathrm{CDCl}_{3}, 100 \mathrm{MHz}\right) \delta 143.8,143.0,132.8,129.1,128.9,128.0,127.6$, $127.2,127.1,126.2,79.6,53.2,25.8,20.8,18.1,13.9,-4.7,-5.4$.

To a solution of S8 (160.5 $\mathrm{mg}, 0.44 \mathrm{mmol})$ was dissolved in dry dichloromethane $(14 \mathrm{ml})$ and methanol $(15 \mathrm{~mL})$ and cooled down to $-78^{\circ} \mathrm{C}$. A stream of ozone was bubbled through until solution turned blue, then $\mathrm{N}_{2}$ was bubbled through until all the blue color disappeared. Sodium borohydride ( $82.3 \mathrm{mg}, 2.1 \mathrm{mmol}, 5$ equiv) was added in four equal portions every 15 minutes for the duration of one hour, while maintaining the temperature at $-78^{\circ} \mathrm{C}$. The reaction was left to stir at room temperature until complete by TLC analysis. The solvent was removed under reduced pressure. The residue was retaken in chloroform $(25 \mathrm{~mL})$ and washed with brine. The aqueous wash was extracted with chloroform $(3 \times 25 \mathrm{~mL})$, and the combined organic layers were dried with $\mathrm{MgSO}_{4}$, filtered, and the solvent was removed under reduced pressure to give an oil. ${ }^{1} \mathrm{H}$ NMR analysis of the crude product showed somehow 3-(tert-butyldimethylsilyloxy)-2,3-diphenyl1-propanal (S9), which was directly used for next step without further purification. 
To a solution of 3-(tert-butyldimethylsilyloxy)-2,3-diphenyl-1-propanal (S9) (57.4 mg, 0.17 mmol) was dissolved in methanol $(1 \mathrm{~mL})$ and sodium borohydride ( $31.6 \mathrm{mg}, 0.84 \mathrm{mmol}, 5$ equiv) was added in four equal every 15 minutes for the duration of one hour. Upon completion, water $(10 \mathrm{~mL})$ was added to the mixture and the whole was extracted with EtOAc $(10 \mathrm{~mL})$ three times and washed with brine, and concentrated in vacuo. The residue was purified by silica gel column chromatography $\left(\mathrm{R}_{f} 0.26\right.$, EtOAc/hexane $=1 / 4$, TLC stain: $p$-anisaldehyde $)$ to provide $(2 R, 3 S)-3$ (tert-Butyldimethylsilyloxy)-2,3-diphenylpropan-1-ol (S10) as a colorless oil (36.5 $\mathrm{mg}, 64 \%)$.

${ }^{1} \mathbf{H}$ NMR $\left(\mathrm{CDCl}_{3}, 500 \mathrm{MHz}\right) \delta 7.26-7.19(\mathrm{~m}, 6 \mathrm{H}), 7.09-7.04(\mathrm{~m}, 4 \mathrm{H}), 4.95(\mathrm{~d}, J=6.0 \mathrm{~Hz}, 1 \mathrm{H})$, $3.86(\mathrm{~m}, 1 \mathrm{H}), 3.78(\mathrm{~m}, 1 \mathrm{H}), 3.13(\mathrm{q}, J=6.5 \mathrm{~Hz}, 1 \mathrm{H}), 1.79(\mathrm{br} \mathrm{s}, 1 \mathrm{H}), 0.80(\mathrm{~s}, 9 \mathrm{H}),-0.10(\mathrm{~s}, 3 \mathrm{H})$, $0.28(\mathrm{~s}, 3 \mathrm{H}) ;{ }^{13} \mathbf{C} \mathbf{N M R}\left(\mathrm{CDCl}_{3}, 100 \mathrm{MHz}\right) \delta 142.5,139.1,129.5,128.2,127.9,127.4,127.0$, $126.9,77.3,63.9,56.6,25.8,18.2,-4.6,-5.4$.

The spectral data of product are consistent with those of the literature. ${ }^{[26]}$

$(2 R, 3 S)$-3-(tert-Butyldimethylsilyloxy)-2,3-diphenylpropan-1-ol: $[\alpha]_{D}^{25}=-53.8\left(c=1.05, \mathrm{CHCl}_{3}\right.$, $83 \%$ ee).

Reference for (2S,3R)-3-(tert-Butyldimethylsilyloxy)-2,3-diphenylpropan-1-ol (ent-S10, CAS: 873108-56-6): lit. ${ }^{[26]}[\alpha]_{D}^{25}=+53.7\left(c=1.05, \mathrm{CHCl}_{3}, 84 \% e e\right)$.

\section{General Procedure for the Furan Synthesis Using DMP}

Substrate 5aia (140.2 mg, $0.5 \mathrm{mmol}$ ) and DMP (Dess-Martin Periodinane: $318.1 \mathrm{mg}, 0.75 \mathrm{mmol}$, 1.5 equiv) were weighed into a $10-\mathrm{mL}$ vial equipped with a magnetic stirrer bar and septum. The system was purged with argon, and then dry dichloromethane $(3 \mathrm{~mL})$ and distilled water $(13.5 \mathrm{mg}$, $1.5 \mathrm{mmol}, 3$ equiv) were added. Under argon atmosphere, the septum was replaced with screw cap. The resulting reaction mixture $(0.1 \mathrm{M})$ was stirred at $30{ }^{\circ} \mathrm{C}$ in an oil bath for 48 hours. Saturated aqueous $\mathrm{NaHCO}_{3}(1 \mathrm{~mL})$ and sodium thiosulfate $(1 \mathrm{~mL})$ were added to quench the reaction, and the resulting mixture was stirred for 30 minutes. The separated water layer was extracted with $\mathrm{CHCl}_{3}$, and the combined organic layers were washed with brine and dried over $\mathrm{MgSO}_{4}$. The volatile material was removed under reduced pressure. The resulting residue was purified by column chromatography to give $\mathbf{8 a}\left(126.6 \mathrm{mg}, 91 \%\right.$ yield, $\mathrm{R}_{f} 0.66$, EtOAc/hexane $\left.=3 / 7\right)$ as a colorless oil.<smiles>CCc1cc(-c2ccccc2)c(-c2ccccc2OC)o1</smiles>

2-(2-Methoxyphenyl)-3-phenyl-5-ethylfuran (8a)

${ }^{1} \mathbf{H}$ NMR $\left(\mathrm{CDCl}_{3}, 400 \mathrm{MHz}\right) \delta 7.44(\mathrm{dd}, J=1.2,7.2 \mathrm{~Hz}, 1 \mathrm{H}), 7.31(\mathrm{dt}, J=1.6,7.6 \mathrm{~Hz}, 1 \mathrm{H}), 7.27-$ $7.14(\mathrm{~m}, 5 \mathrm{H}), 6.97(\mathrm{t}, J=7.2 \mathrm{~Hz}, 1 \mathrm{H}), 6.87(\mathrm{~d}, J=8.0 \mathrm{~Hz}, 1 \mathrm{H}), 6.31(\mathrm{~s}, 1 \mathrm{H}), 3.45(\mathrm{~s}, 3 \mathrm{H}), 2.73(\mathrm{q}$, $J=7.6 \mathrm{~Hz}, 2 \mathrm{H}), 1.31(\mathrm{t}, J=7.6 \mathrm{~Hz}, 3 \mathrm{H}) ;{ }^{13} \mathbf{C ~ N M R}\left(\mathrm{CDCl}_{3}, 100 \mathrm{MHz}\right) \delta 157.5,156.8,144.4$, $135.2,131.1,129.6,128.1,127.3,126.3,123.9,121.3,120.7,111.5,106.7,55.2,21.6,12.2$; HRMS: $m / z\left(\mathrm{M}^{+}\right)$calcd for $\mathrm{C}_{19} \mathrm{H}_{18} \mathrm{O}_{2}: 278.1307$, found: 278.1305 . 
<smiles>CCc1cc(-c2cccc(OC)c2)c(-c2ccccc2)o1</smiles>

\section{2-Phenyl-3-(3-methoxyphenyl)-5-ethylfuran (8b)}

Purified by silica gel column chromatography $\left(0.5 \mathrm{mmol}\right.$ scale reaction, $93.2 \mathrm{mg}, 67 \%$ yield, $\mathrm{R}_{f}$ 0.62, EtOAc/hexane = 3/7). Colorless oil, ${ }^{1} \mathbf{H}$ NMR $\left(\mathrm{CDCl}_{3}, 500 \mathrm{MHz}\right) \delta 7.52(\mathrm{~d}, J=8.5 \mathrm{~Hz}, 2 \mathrm{H})$, $7.30-7.16(\mathrm{~m}, 4 \mathrm{H}), 6.99(\mathrm{~d}, J=9.5 \mathrm{~Hz}, 1 \mathrm{H}), 6.95(\mathrm{t}, J=3.0 \mathrm{~Hz}, 1 \mathrm{H}), 6.83(\mathrm{dd}, J=3.5,9.5 \mathrm{~Hz}$, $1 \mathrm{H}), 6.16(\mathrm{~s}, 1 \mathrm{H}), 3.75(\mathrm{~s}, 3 \mathrm{H}), 2.73(\mathrm{q}, J=9.5 \mathrm{~Hz}, 2 \mathrm{H}), 1.31(\mathrm{t}, J=9.5 \mathrm{~Hz}, 3 \mathrm{H}) ;{ }^{13} \mathbf{C} \mathbf{N M R}$ $\left(\mathrm{CDCl}_{3}, 100 \mathrm{MHz}\right) \delta 159.8,157.0,146.8,136.3,131.6,129.7,128.4,127.2,126.2,122.9,121.2$, $114.0,113.0,108.7,55.3,21.5,12.2$.

2.1, 131.5, 128.8, 128.2, 126.7, 125.3, 123.9, 120.8, 119.0, 111.3, 109.6, 55.5, 21.6, 12.2 HRMS: $\mathrm{m} / z\left(\mathrm{M}^{+}\right)$calcd for $\mathrm{C}_{19} \mathrm{H}_{18} \mathrm{O}_{2}: 278.1307$, found: 278.1301 . 


\section{References}

[1] E. Barreiro, A. Sanz-Vidal, E. Tan, S.-H. Lau, T. D. Sheppard, S. Díez-González, Eur. J. Org. Chem. 2015, 2015, 7544-7549.

[2] M. J. Joung, J. H. Ahn, N. M. Yoon, J. Org. Chem. 1996, 61, 4472-4475.

[3] Q. Deng, R. Shen, R. Ding, L. Zhang, Adv. Synth. Catal. 2014, 356, 2931-2936.

[4] L. Eccleshare, L. Lozada-Rodríguez, P. Cooper, L. Burroughs, J. Ritchie, W. Lewis, S. Woodward, Chem. - A Eur. J. 2016, 22, 12542-12547.

[5] R. U. Braun, M. Ansorge, T. J. J. Müller, Chem. - A Eur. J. 2006, 12, 9081-9094.

[6] E. Y. Schmidt, N. A. Cherimichkina, I. A. Bidusenko, N. I. Protzuk, B. A. Trofimov, European J. Org. Chem. 2014, 2014, 4663-4670.

[7] J. Barluenga, F. J. Fañanás, R. Sanz, C. Marcos, Chem. - A Eur. J. 2005, 11, 5397-5407.

[8] A. Hosseini, D. Seidel, A. Miska, P. R. Schreiner, Org. Lett. 2015, 17, 2808-2811.

[9] Y. Nakanishi, K. Miki, K. Ohe, Tetrahedron 2007, 63, 12138-12148.

[10] N. Ghosh, S. Nayak, A. K. Sahoo, J. Org. Chem. 2011, 76, 500-511.

[11] L. Zhao, G. Huang, B. Guo, L. Xu, J. Chen, W. Cao, G. Zhao, X. Wu, Org. Lett. 2014, 16, 5584-5587.

[12] A. Lepronier, T. Achard, L. Giordano, A. Tenaglia, G. Buono, H. Clavier, Adv. Synth. Catal. 2016, 358, 631-642.

[13] C. K. Skepper, J. B. MacMillan, G.-X. Zhou, M. N. Masuno, T. F. Molinski, J. Am. Chem. Soc. 2007, 129, 4150-4151.

[14] Y. Horino, M. Murakami, A. Aimono, J. H. Lee, H. Abe, Org. Lett. 2019, 21, 476-480.

[15] K. Kiyokawa, N. Tachikake, M. Yasuda, A. Baba, Angew. Chem. Int. Ed. 2011, 50, 1039310396.

[16] K. Jones, M. F. Lappert, J. Chem. Soc. 1965, 1944.

[17] Y. Zhu, D. B. Millet, M. O. Wolf, S. J. Rettig, Organometallics 1999, 18, 1930-1938.

[18] E. Viola, C. Lo Sterzo, R. Crescenzi, G. Frachey, J. Organomet. Chem. 1995, 493, C9-C13.

[19] C. J. Elsevier, P. Vermeer, J. Org. Chem 1989, 54, 3726-3730.

[20] J. F. Reichwein, B. L. Pagenkopf, J. Org. Chem. 2003, 68, 1459-1463.

[21] T. Kawajiri, R. Ohta, H. Fujioka, H. Sajiki, Y. Sawama, Chem. Commun. 2018, 54, 374 377.

[22] A. R. Patel, X.-G. Hu, A. Lawer, M. I. Ahmed, C. Au, R. Jwad, J. Trinh, C. Gonzalez, E. Hannah, M. M. Bhadbhade, et al., Tetrahedron 2016, 72, 3305-3317.

[23] S. K. Cherian, P. Kumar, Tetrahedron: Asymmetry 2007, 18, 982-987.

[24] J. S. Yadav, G. Rajaiah, A. K. Raju, Tetrahedron Lett. 2003, 44, 5831-5833.

[25] Y. Tu, Z. Wang, Y. Shi, J. Am. Chem. Soc. 1996, 118, 9806-9807.

[26] H. M. L. Davies, S. J. Hedley, B. R. Bohall, J. Org. Chem. 2005, 70, 10737-10742. 


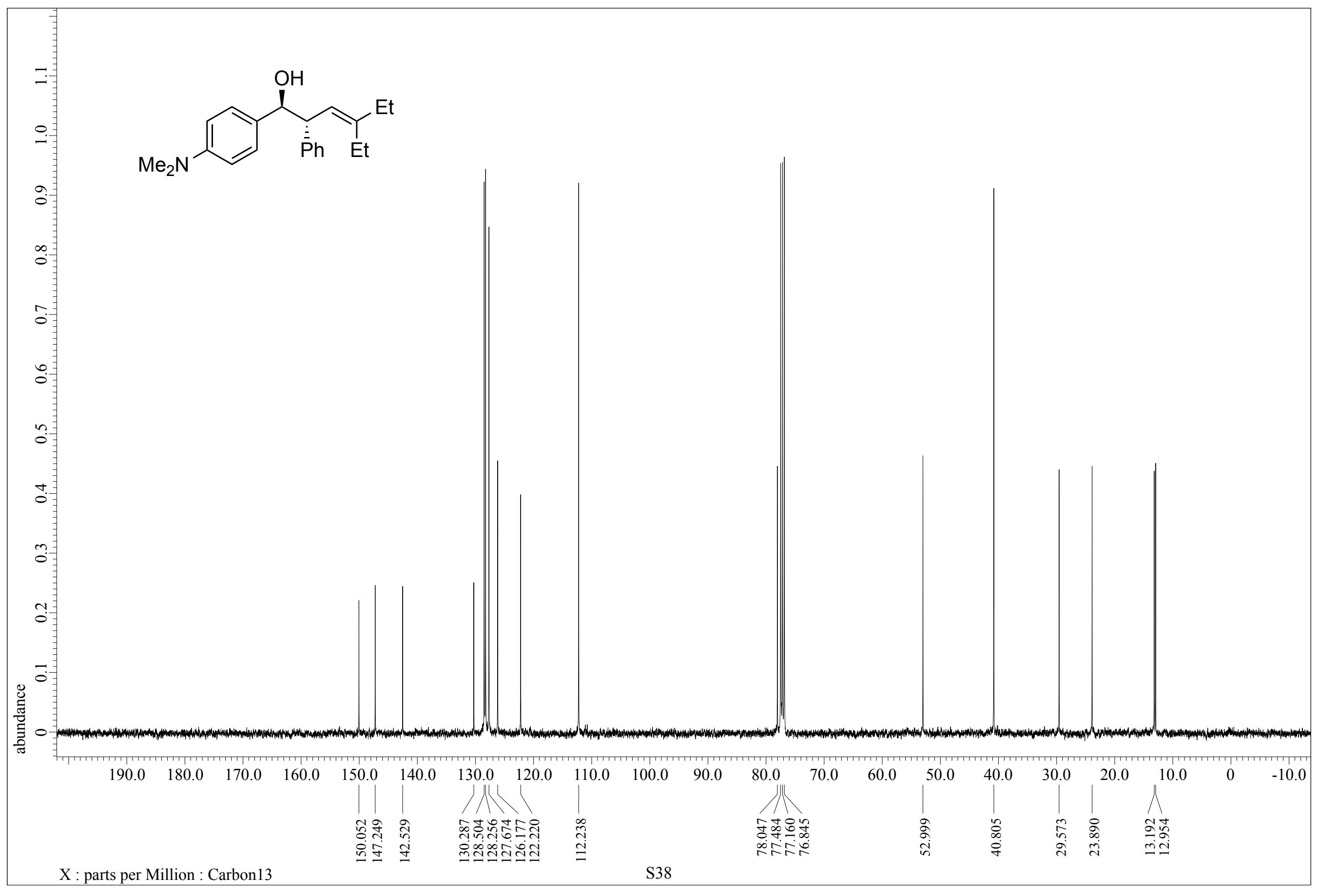




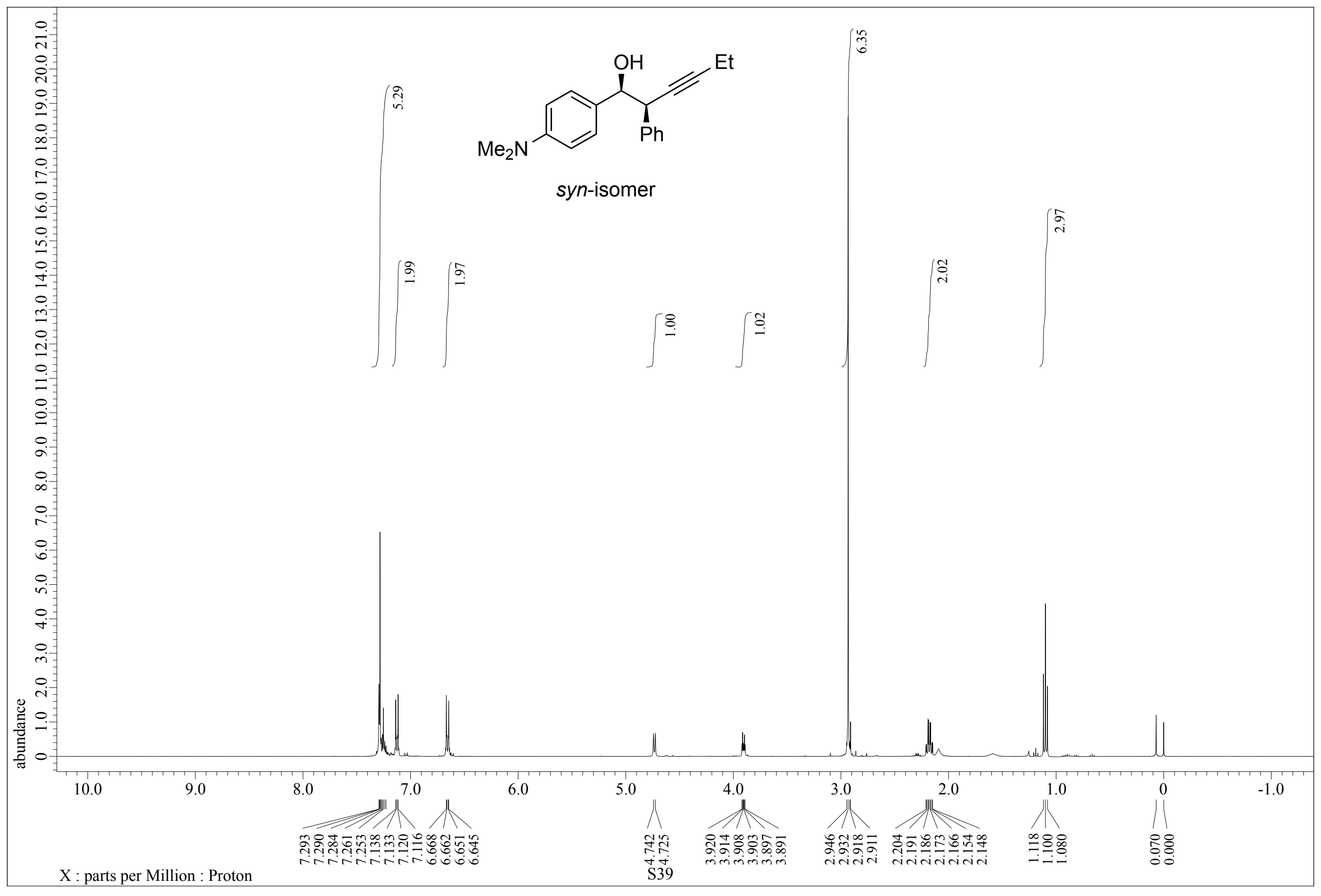




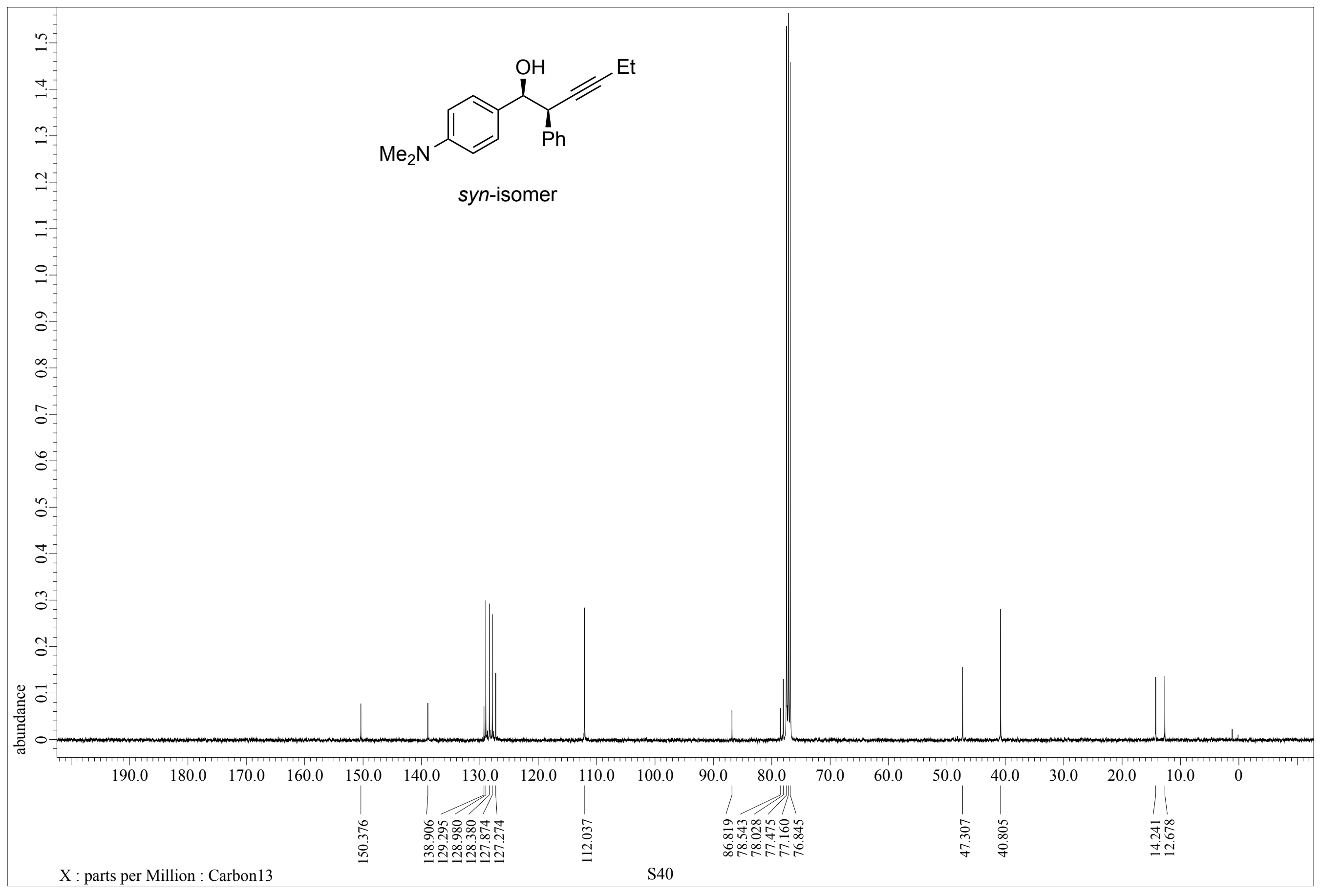




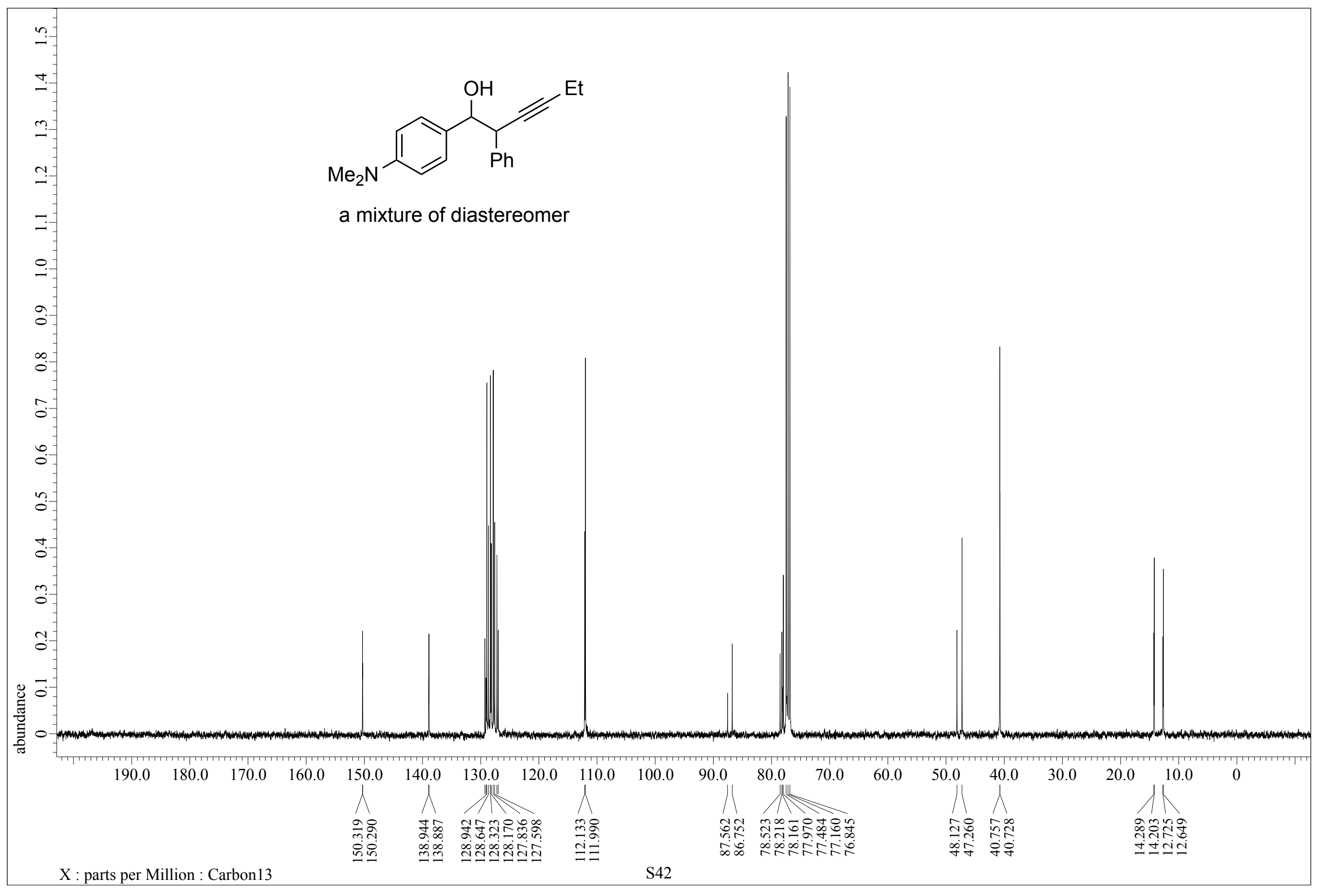




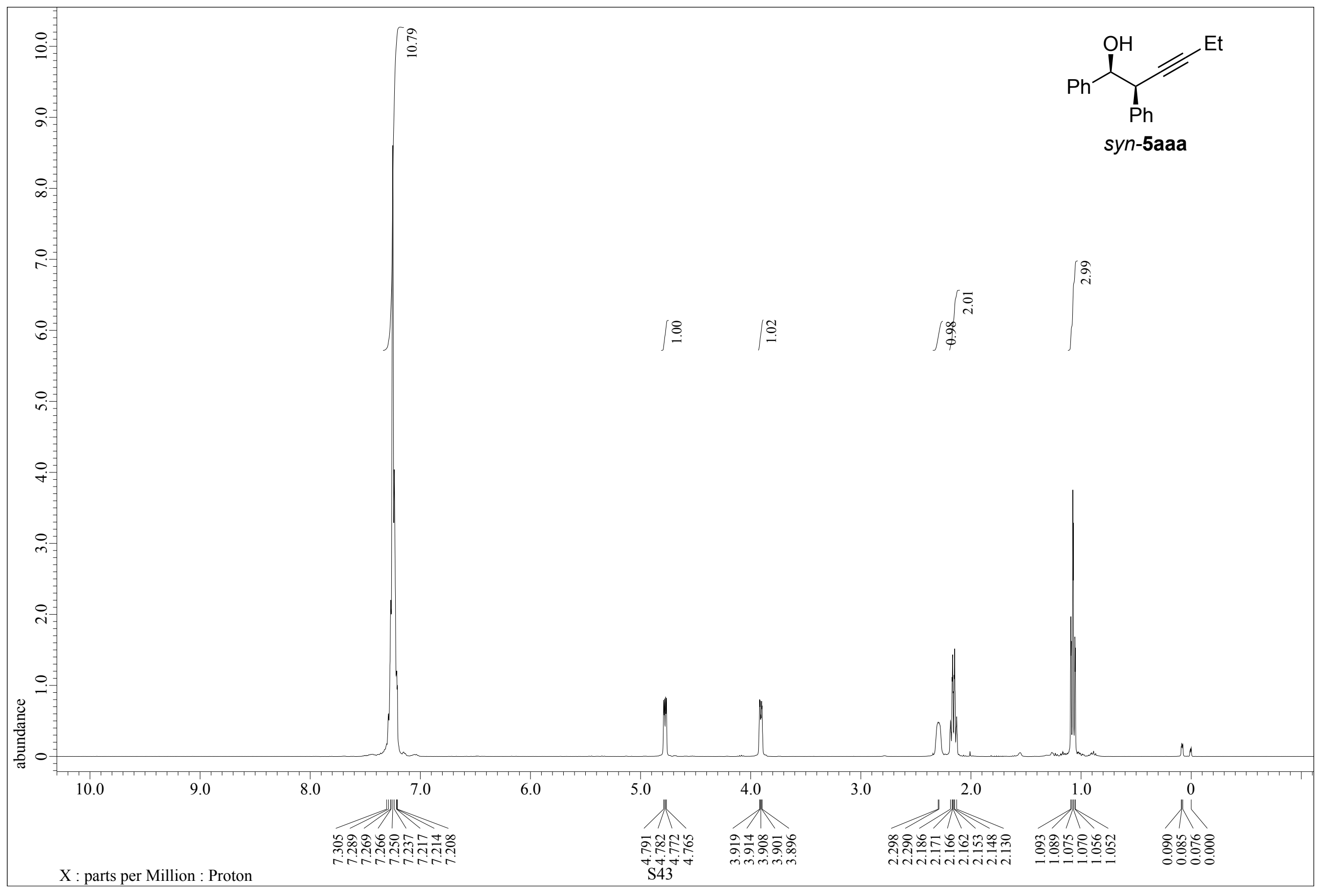




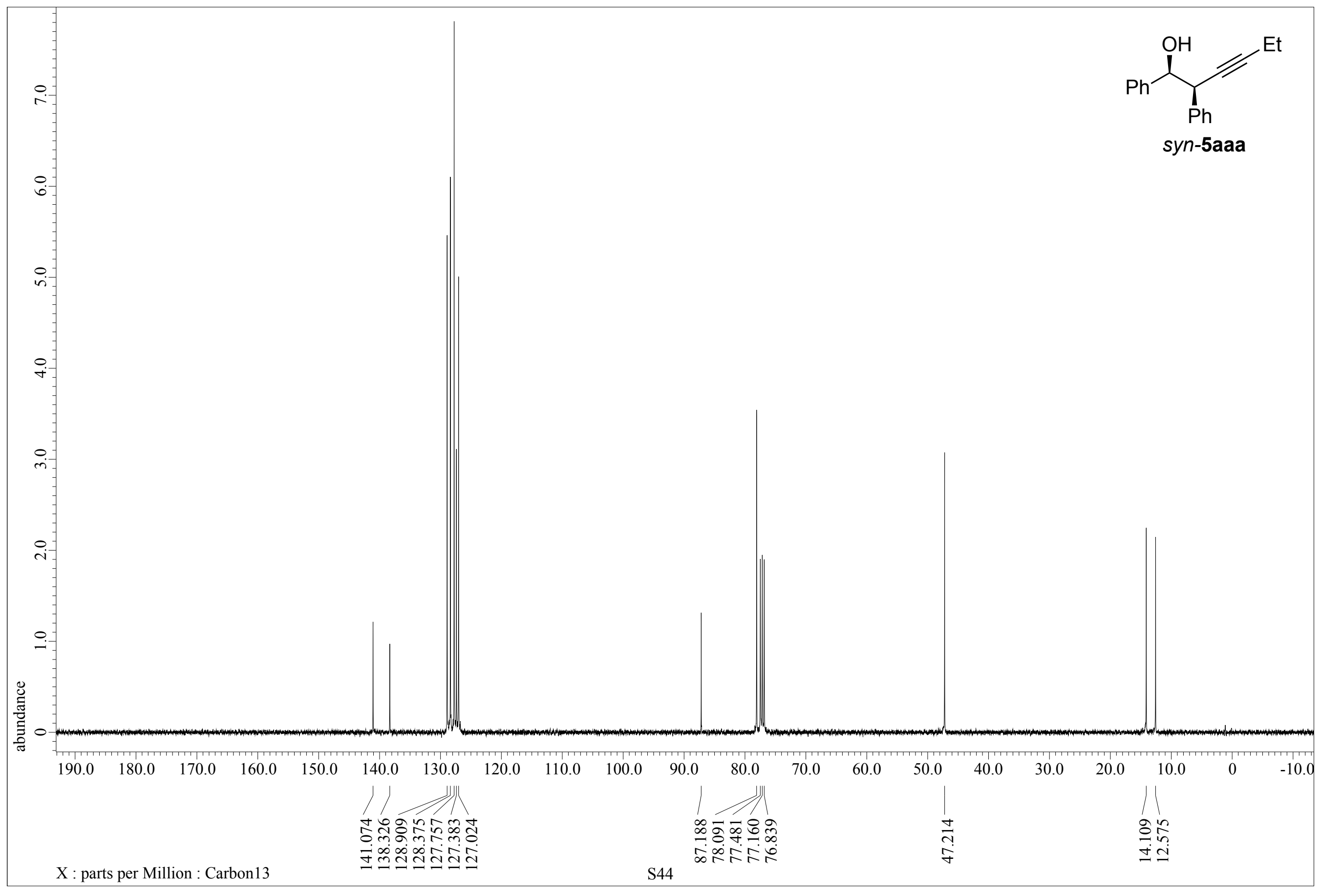




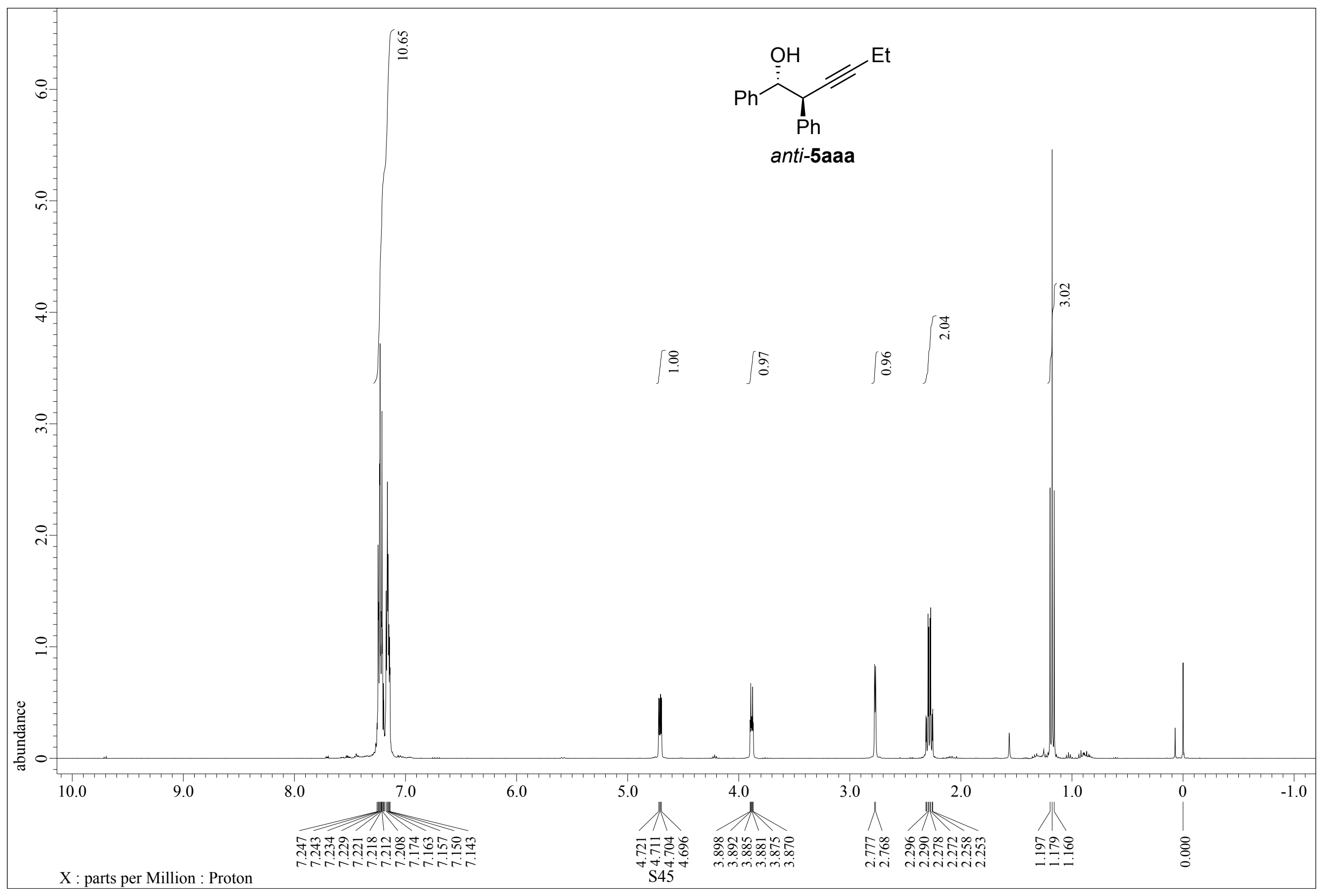




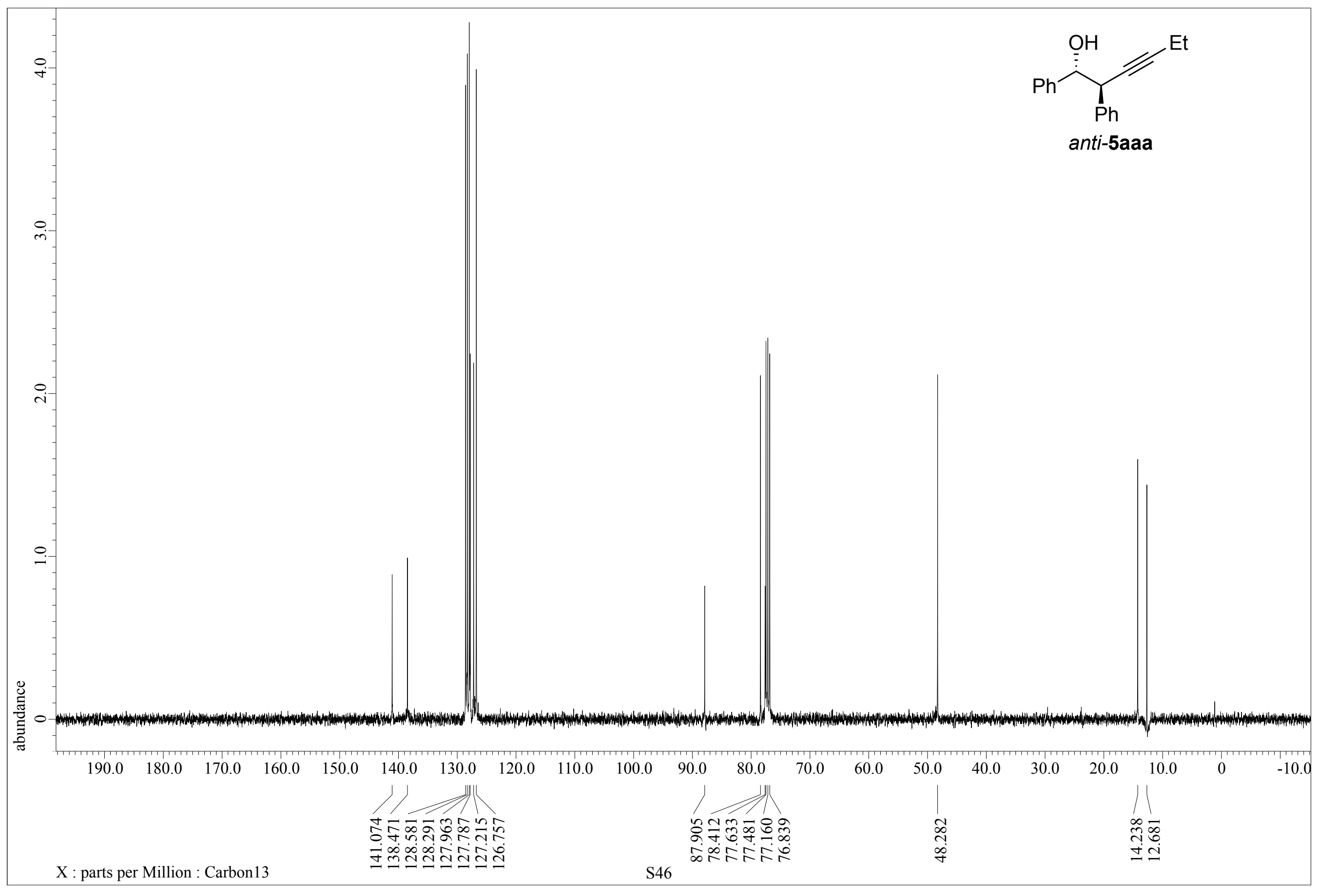




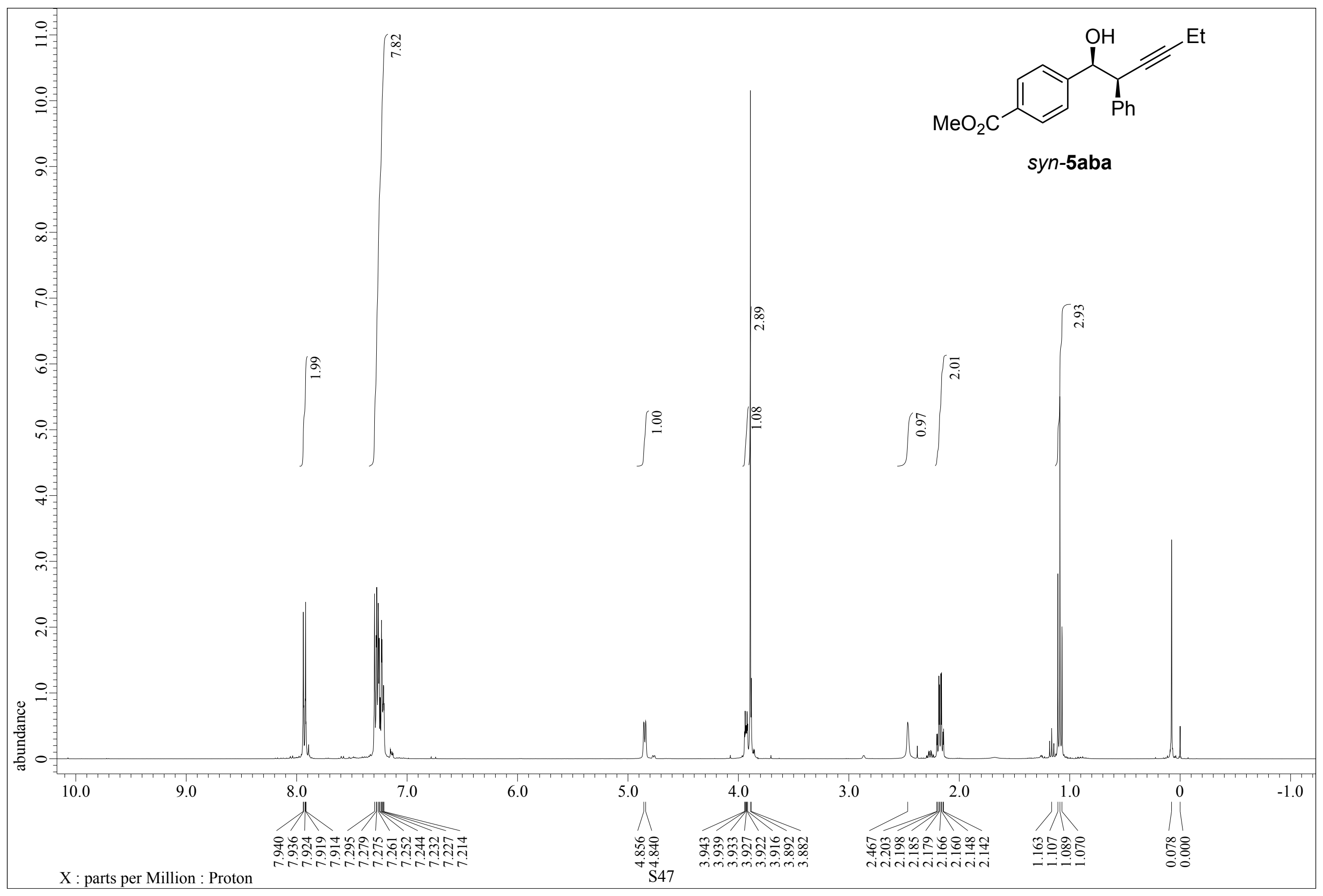




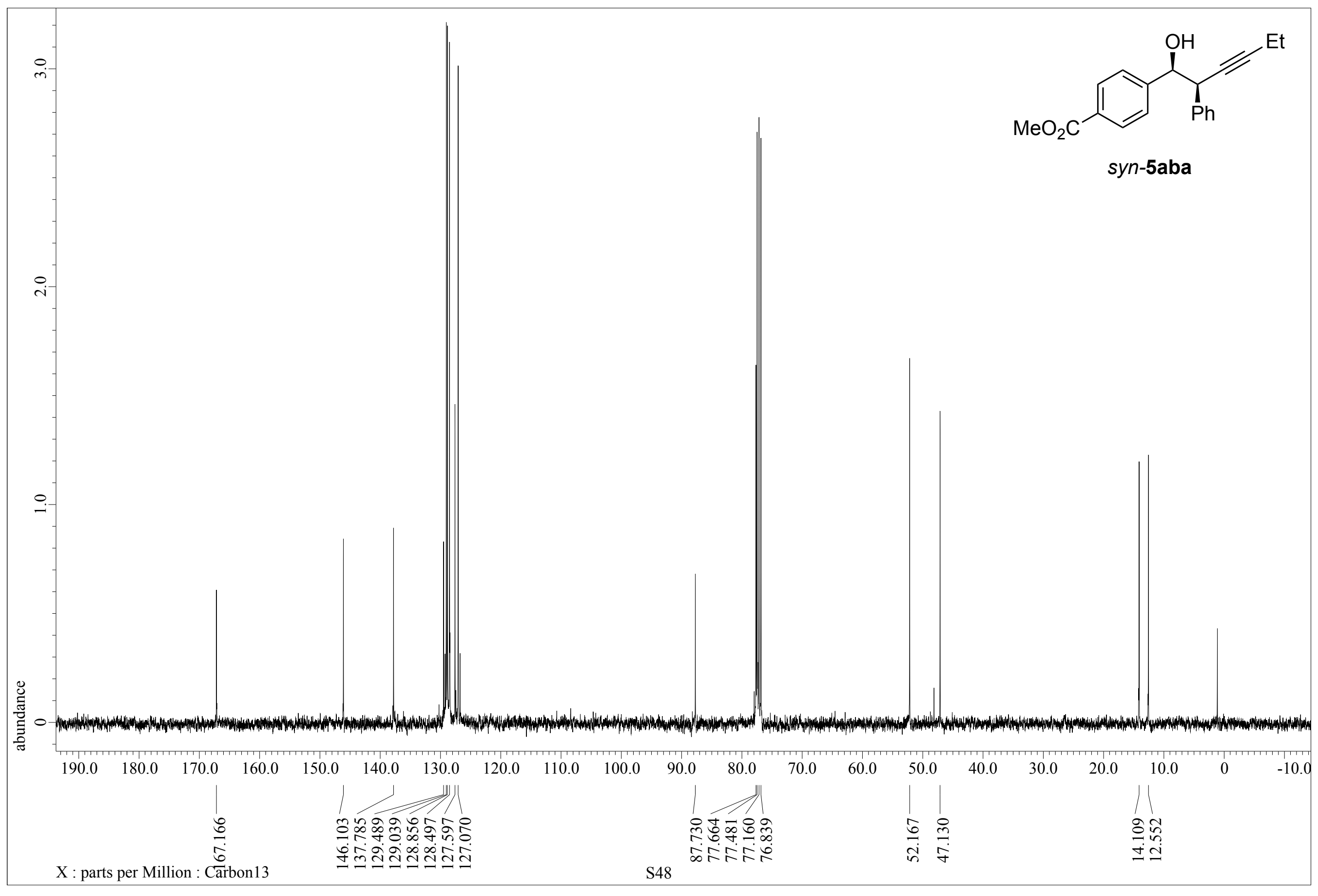




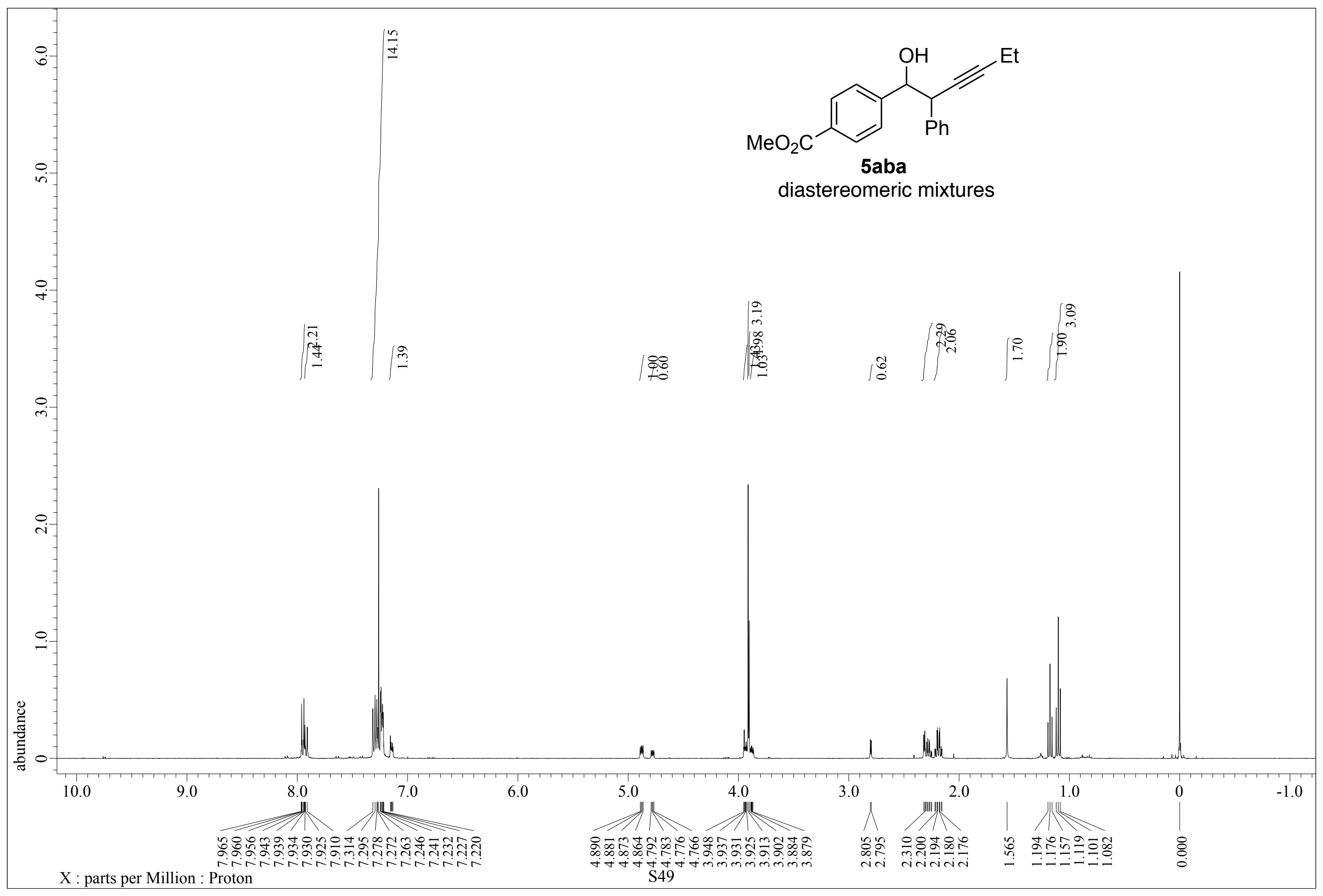




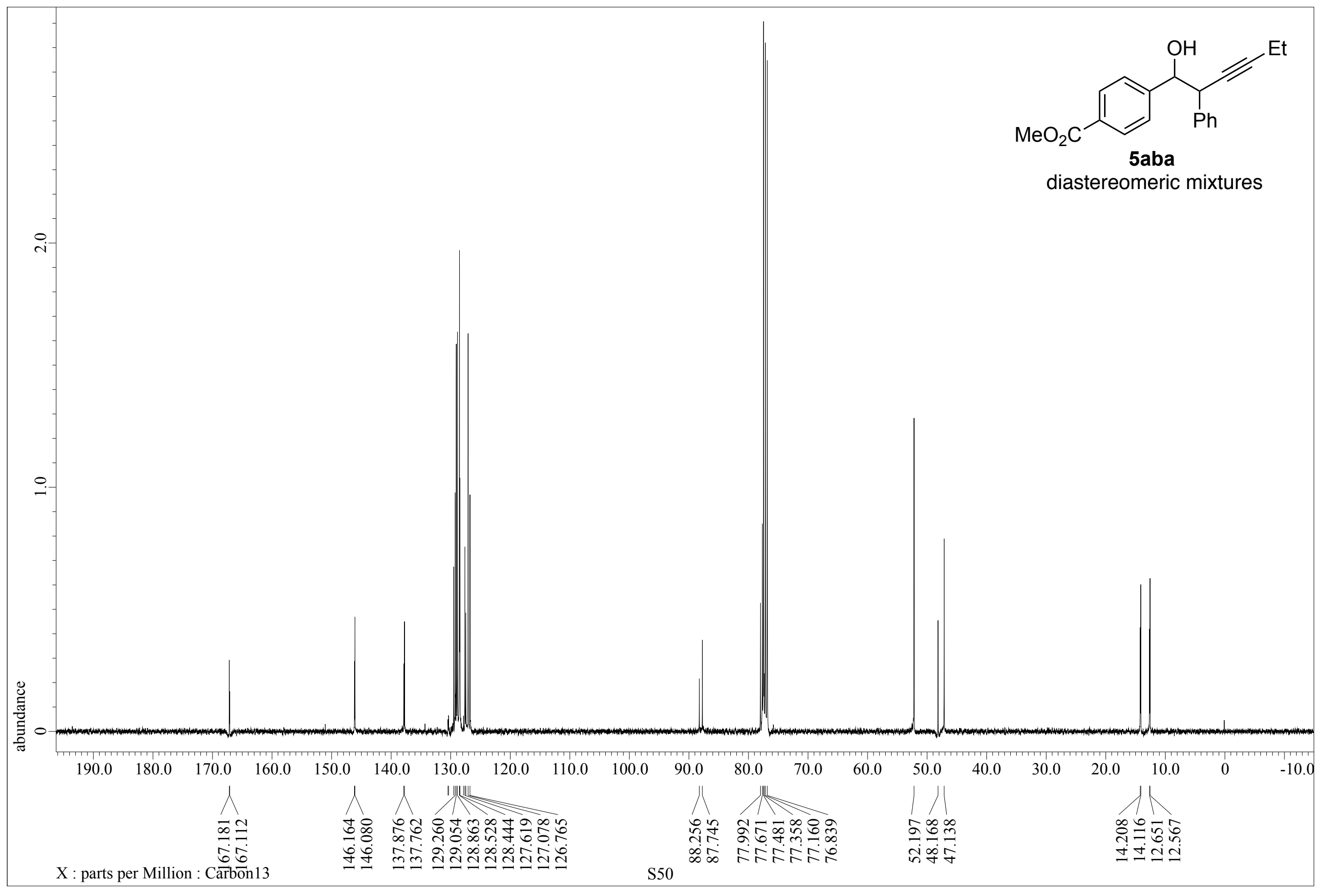




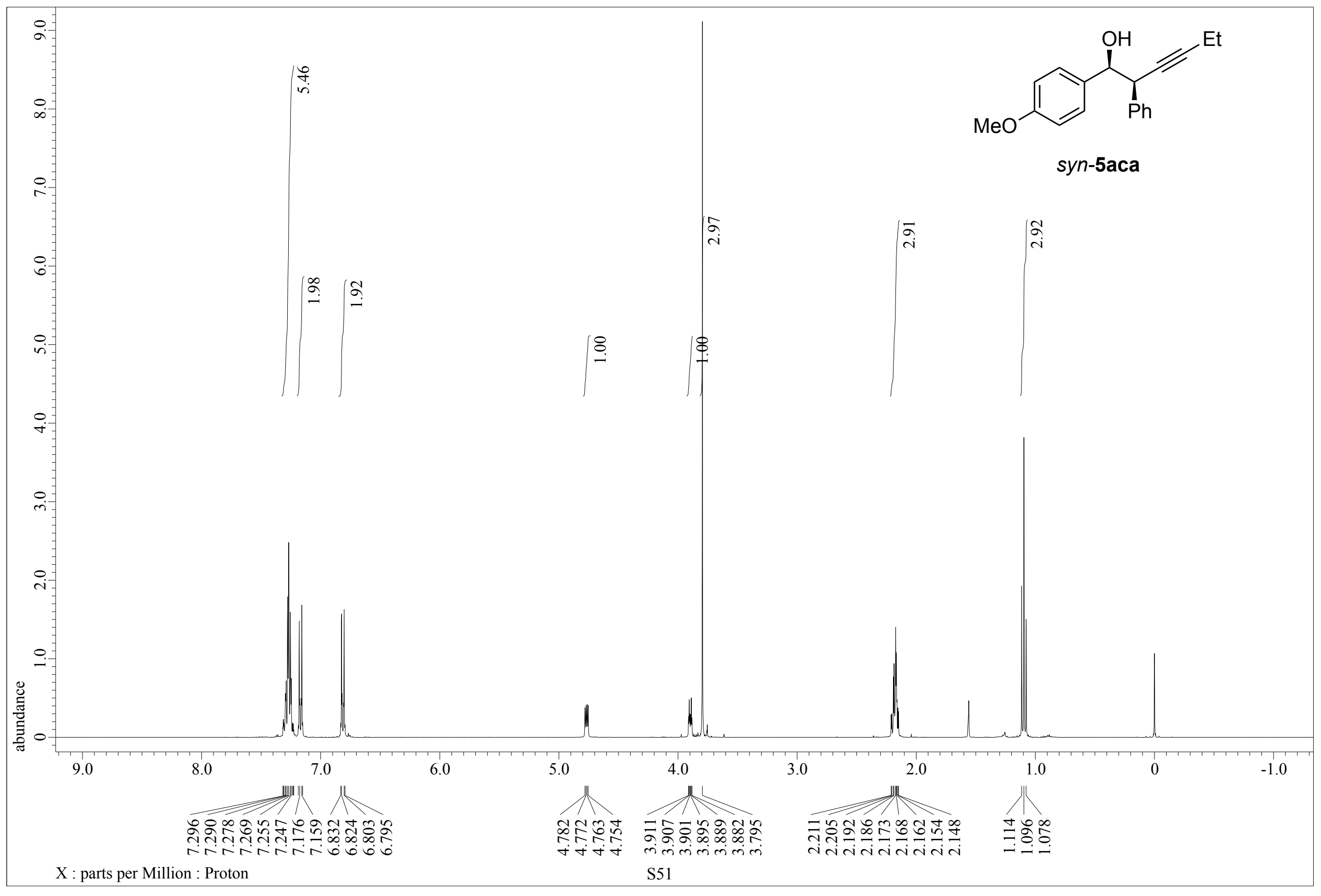




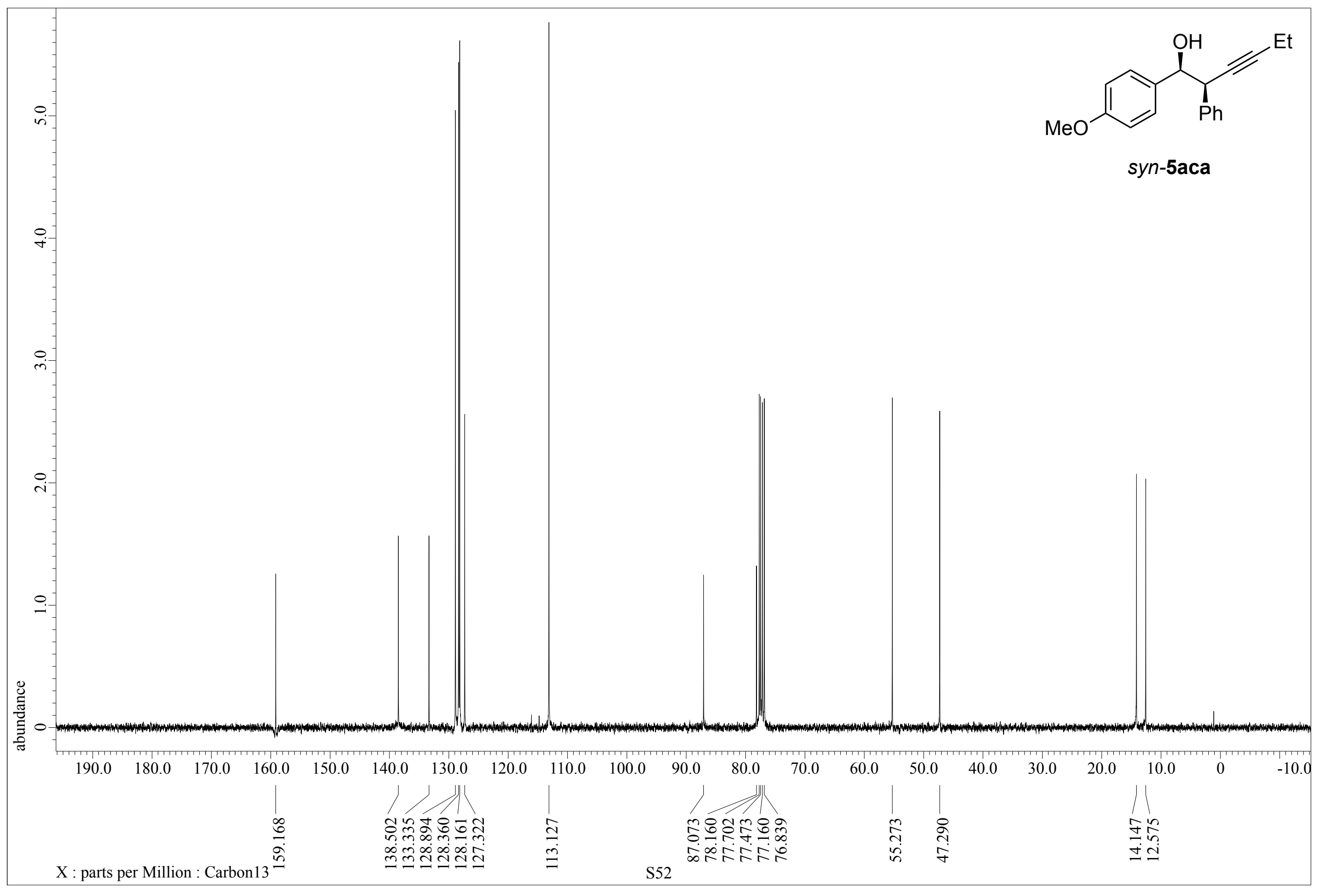




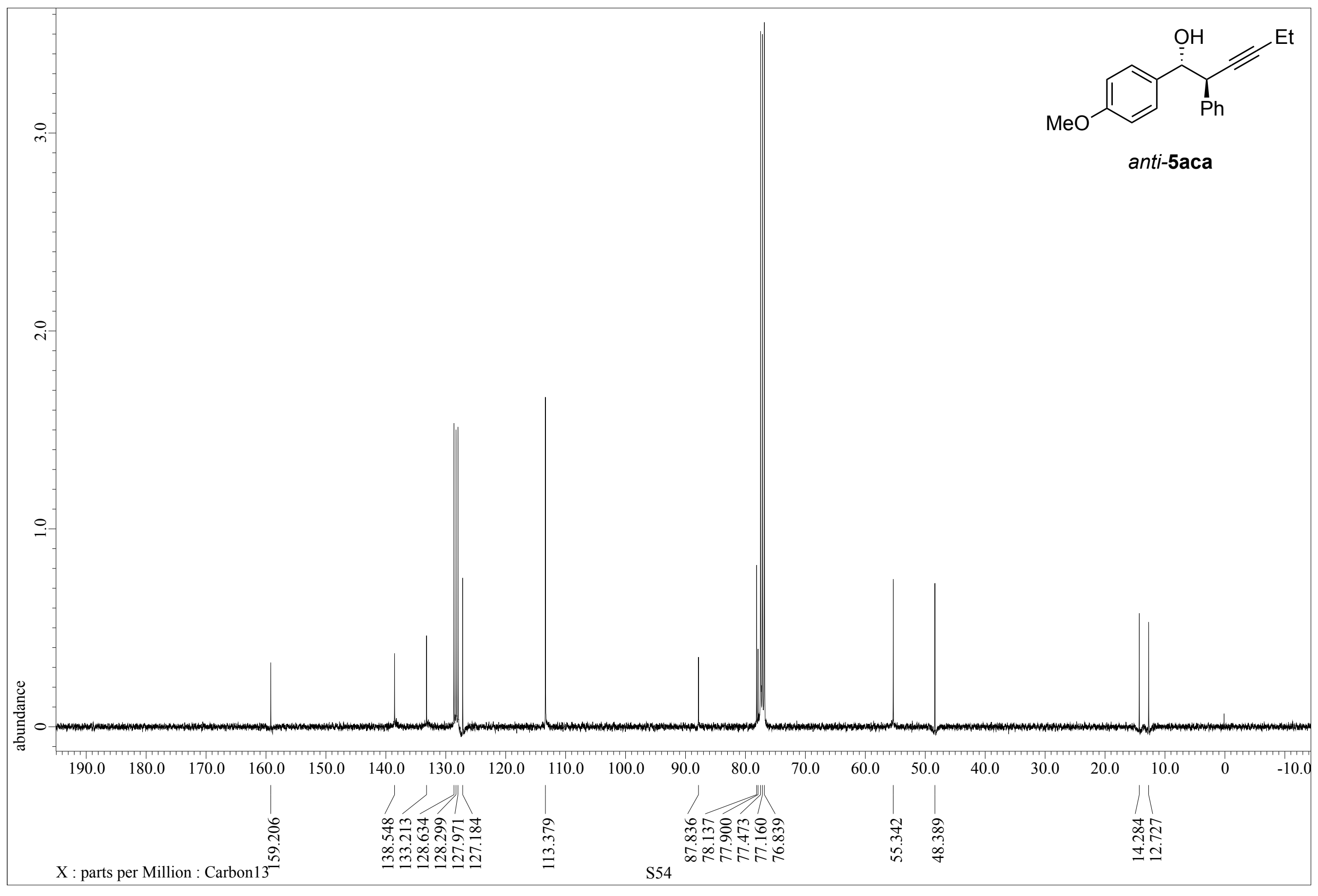




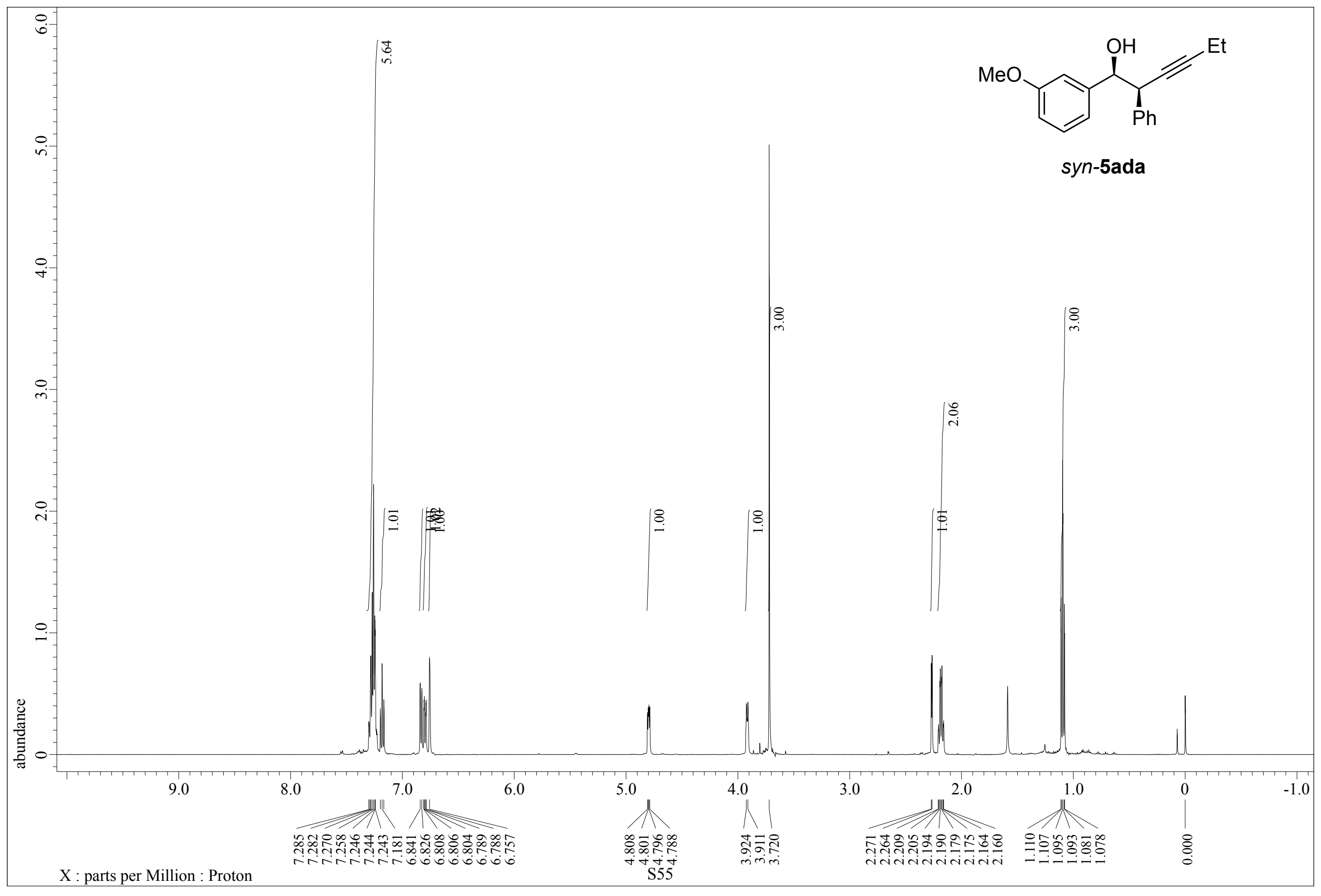




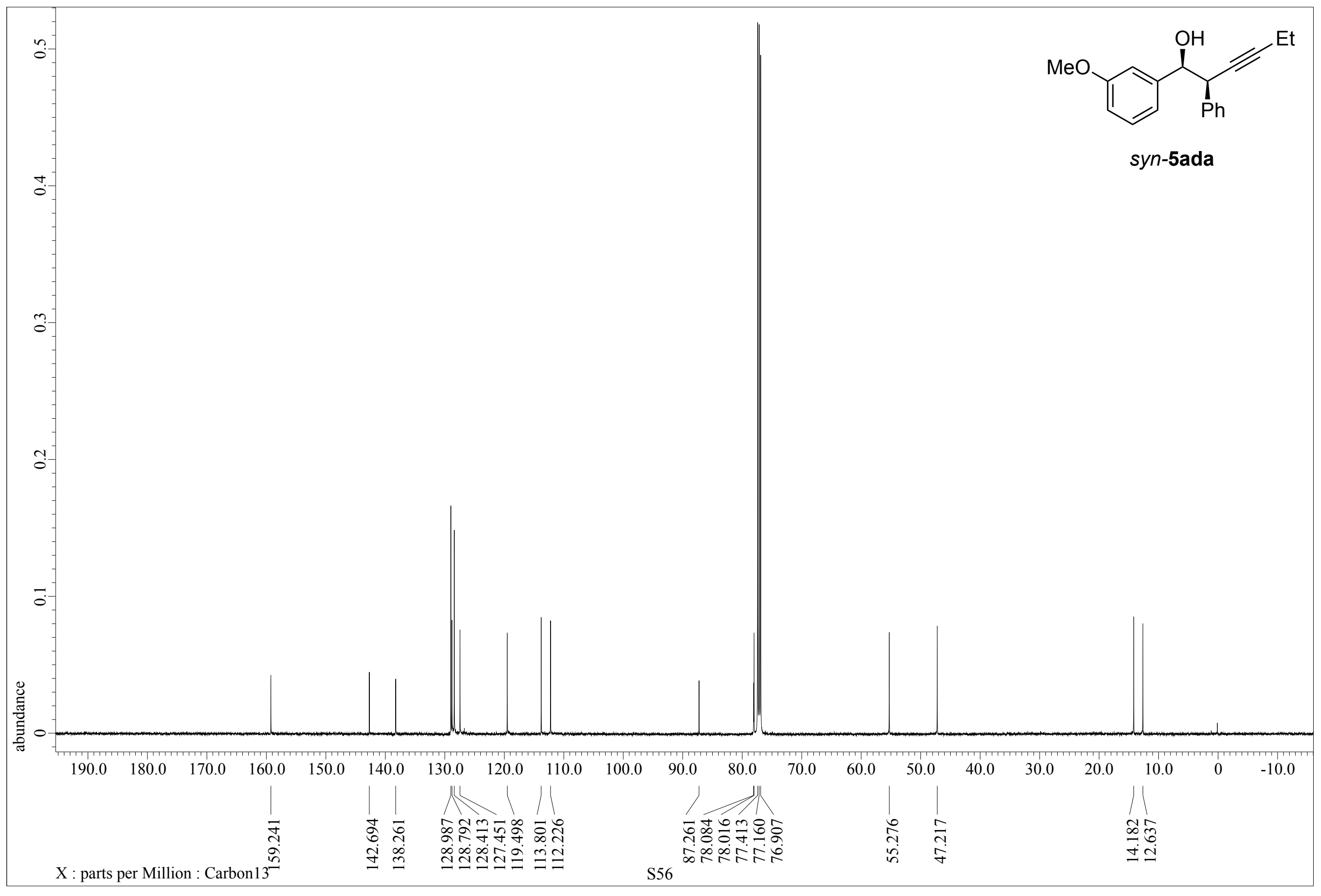




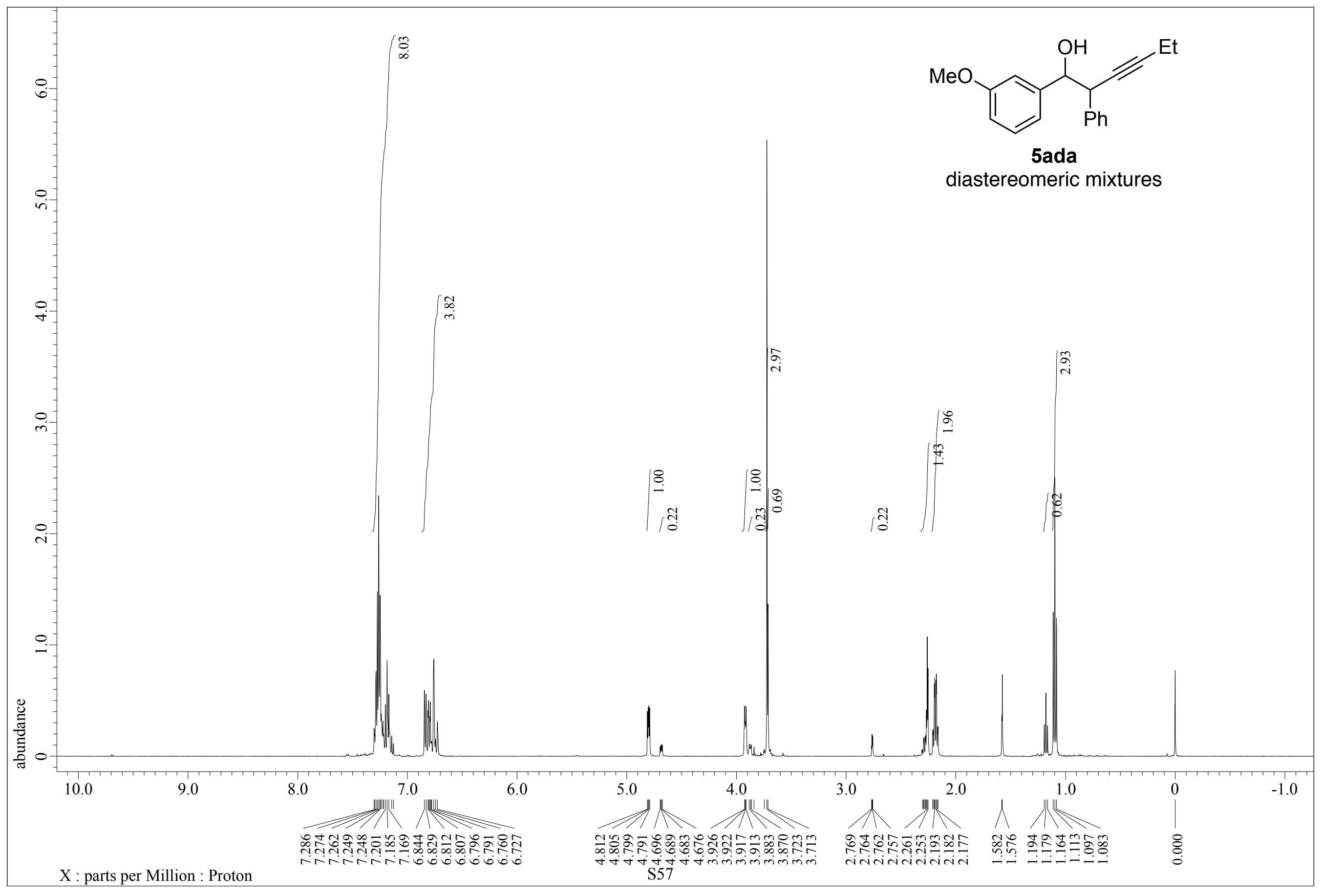




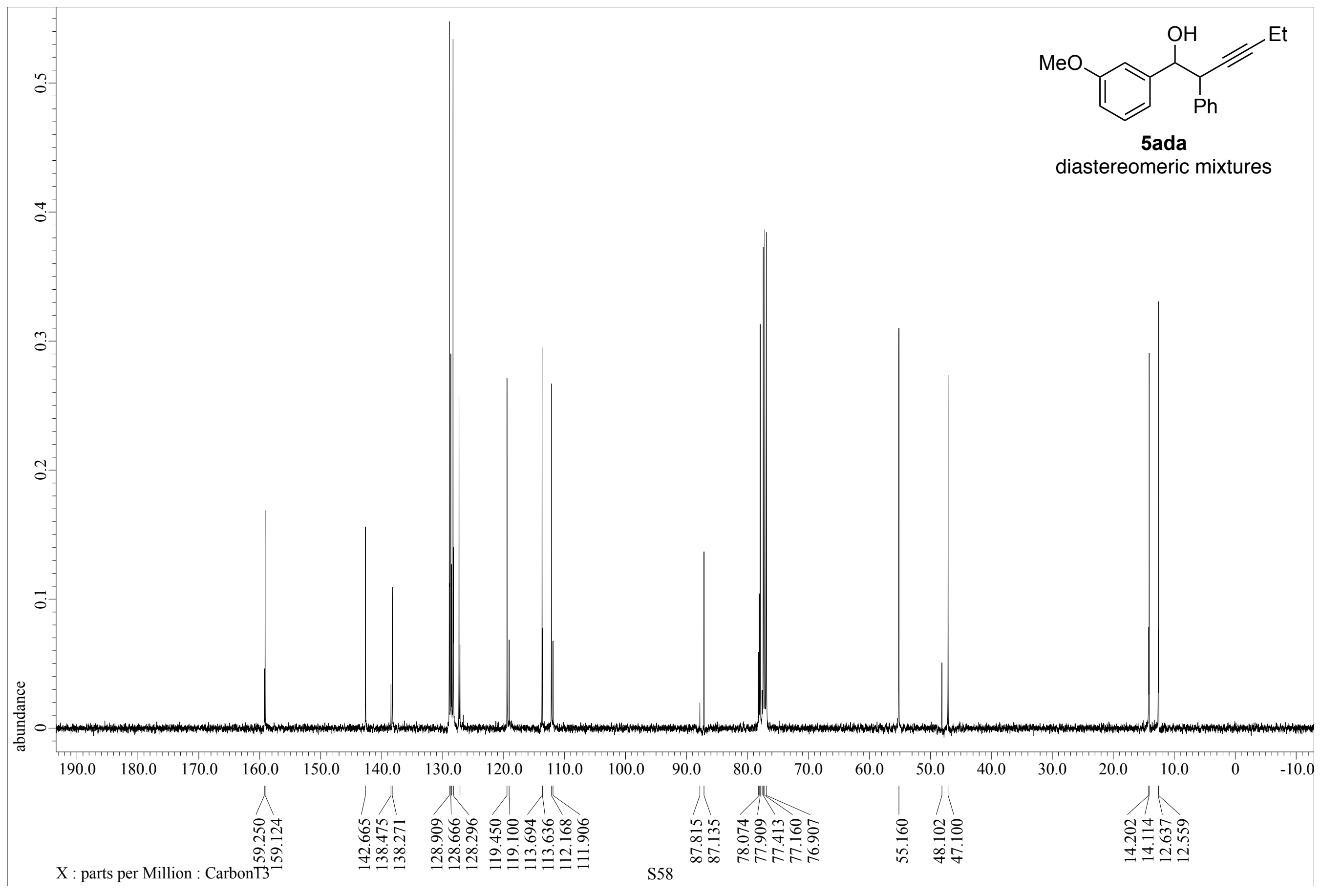




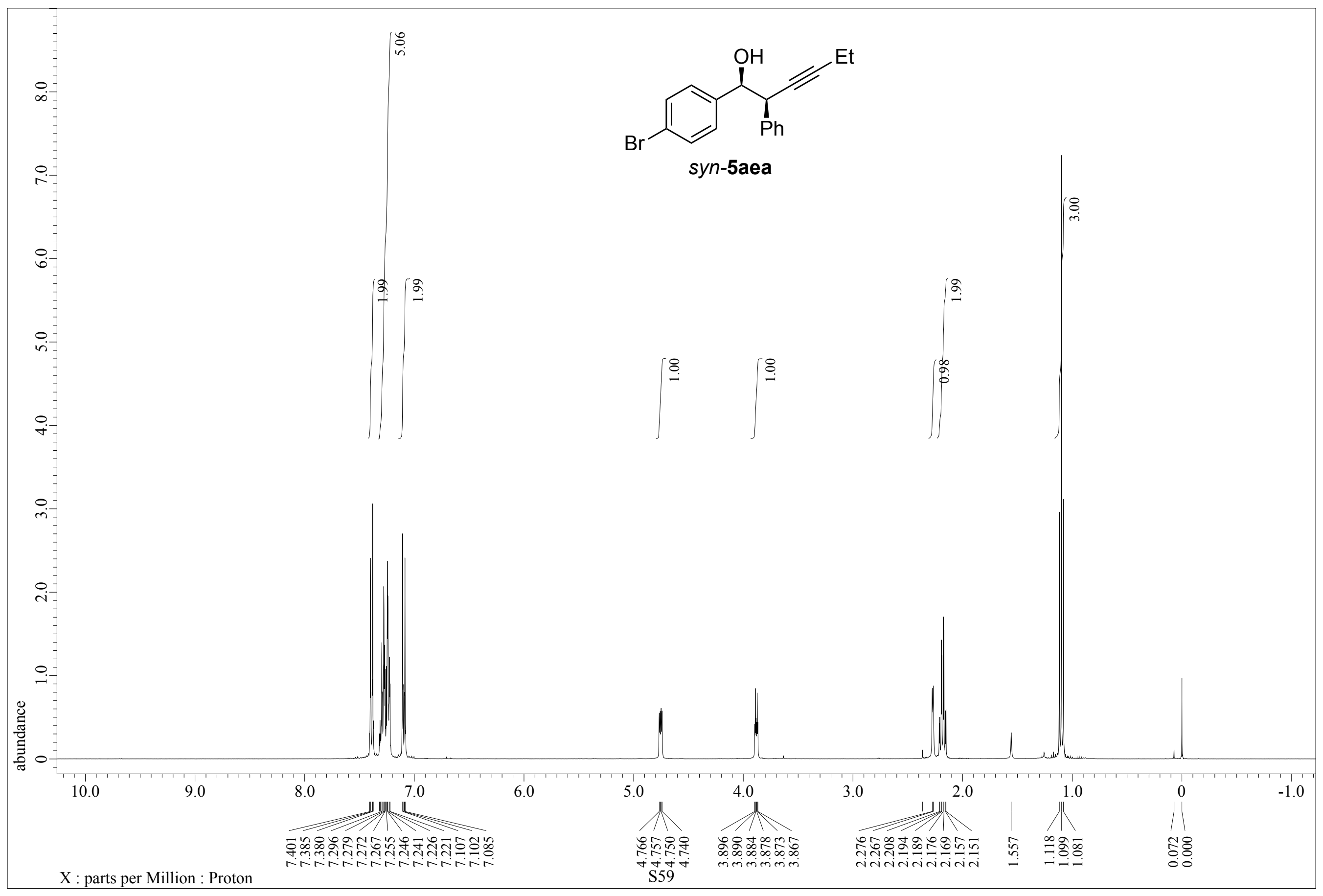




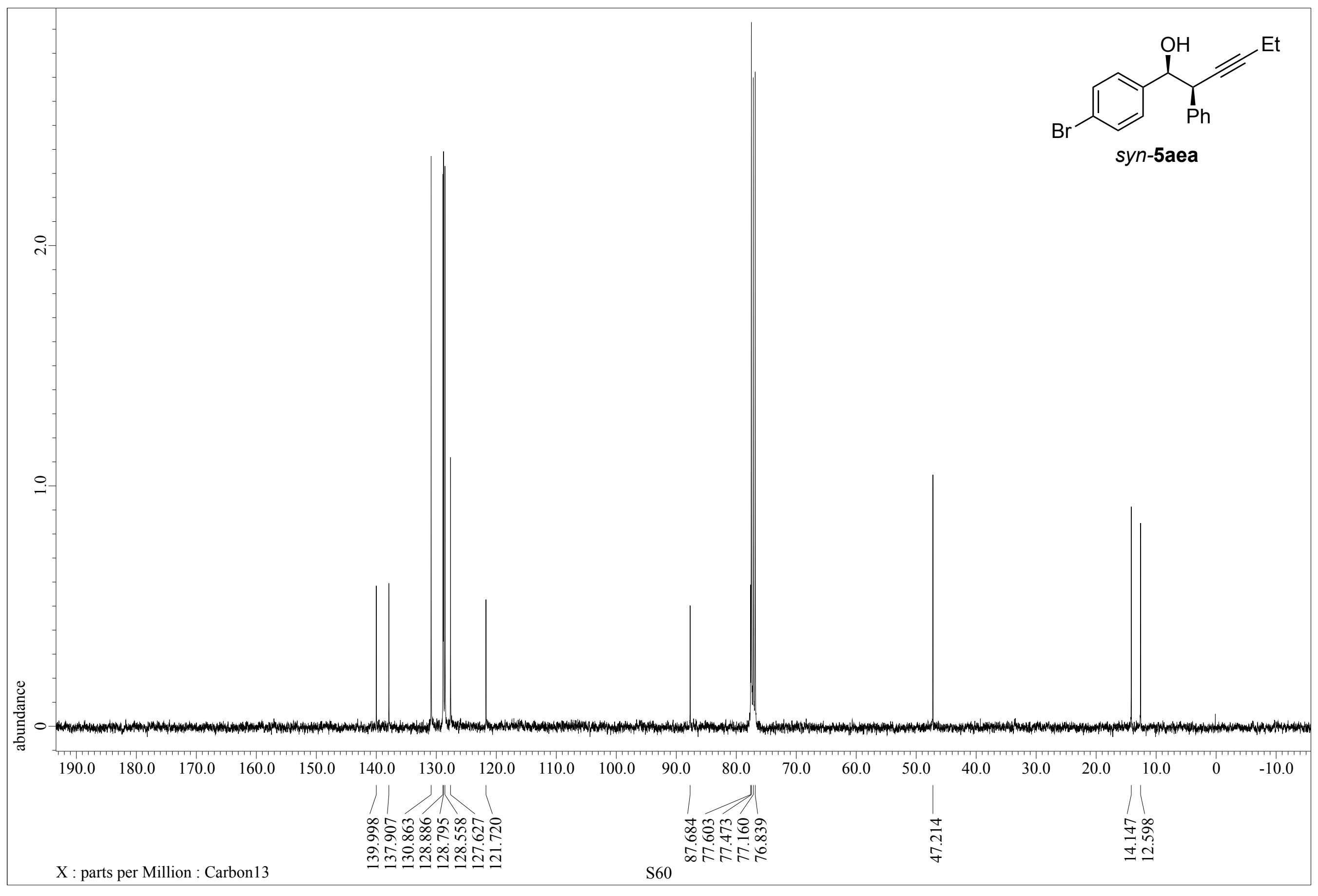




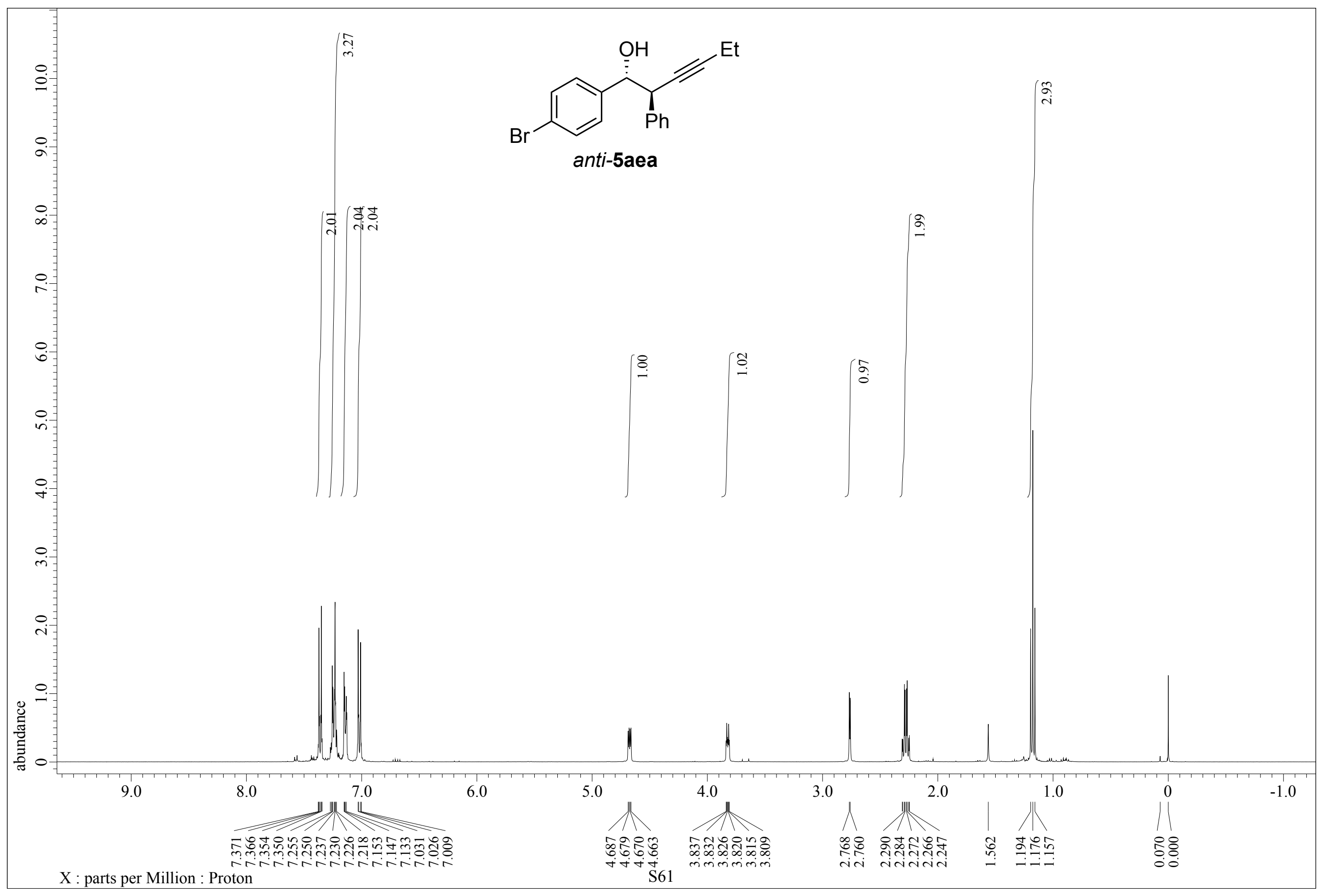




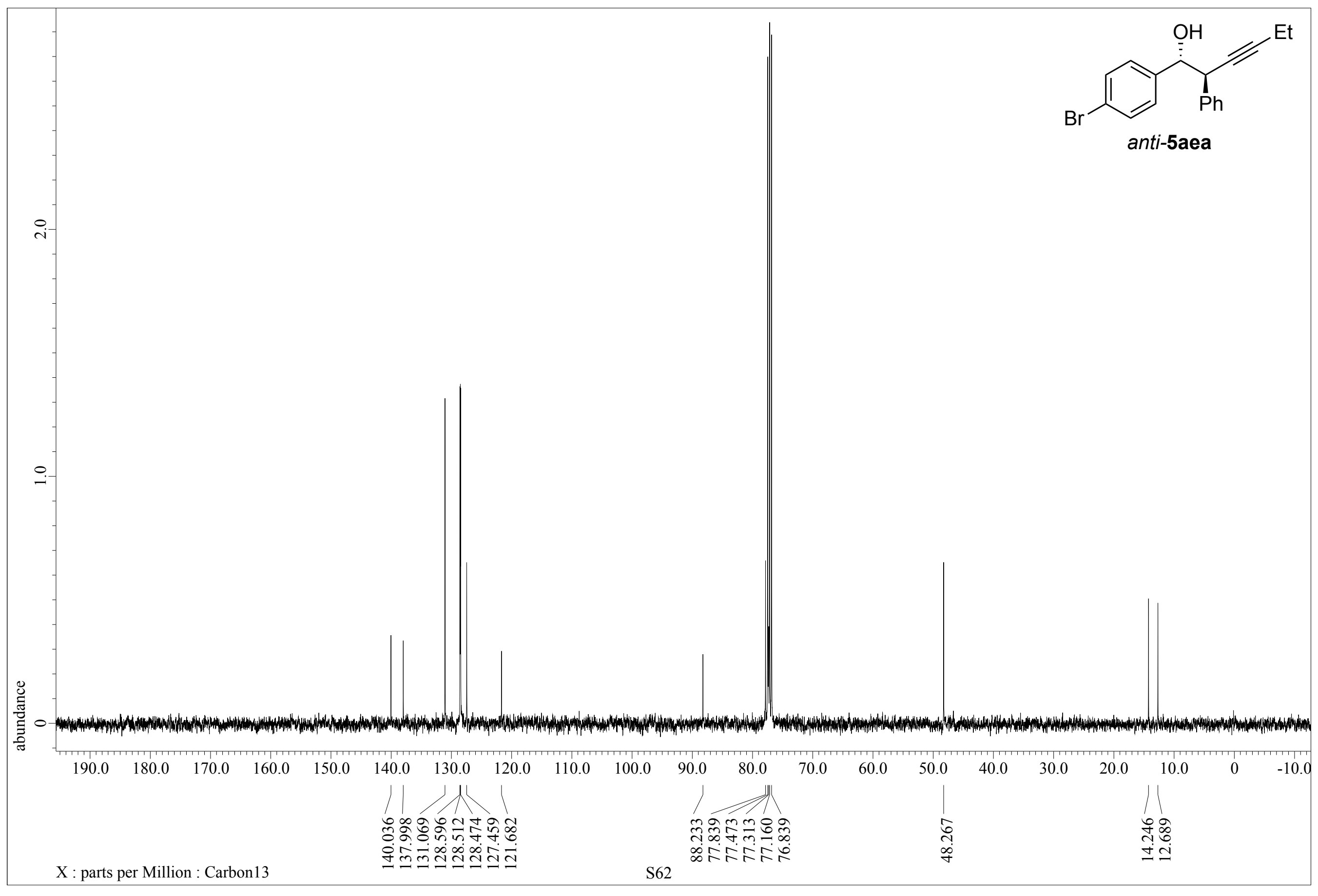




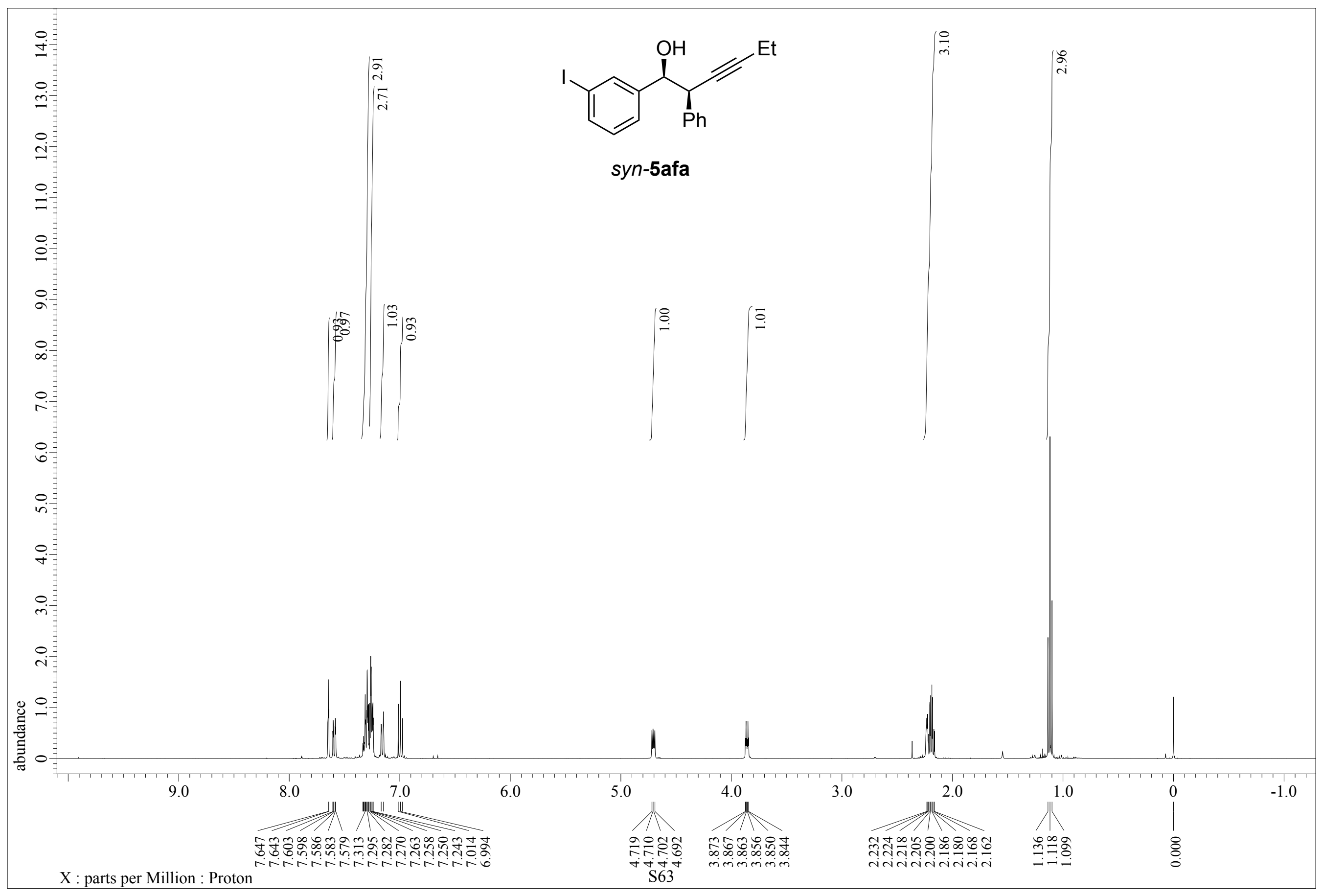




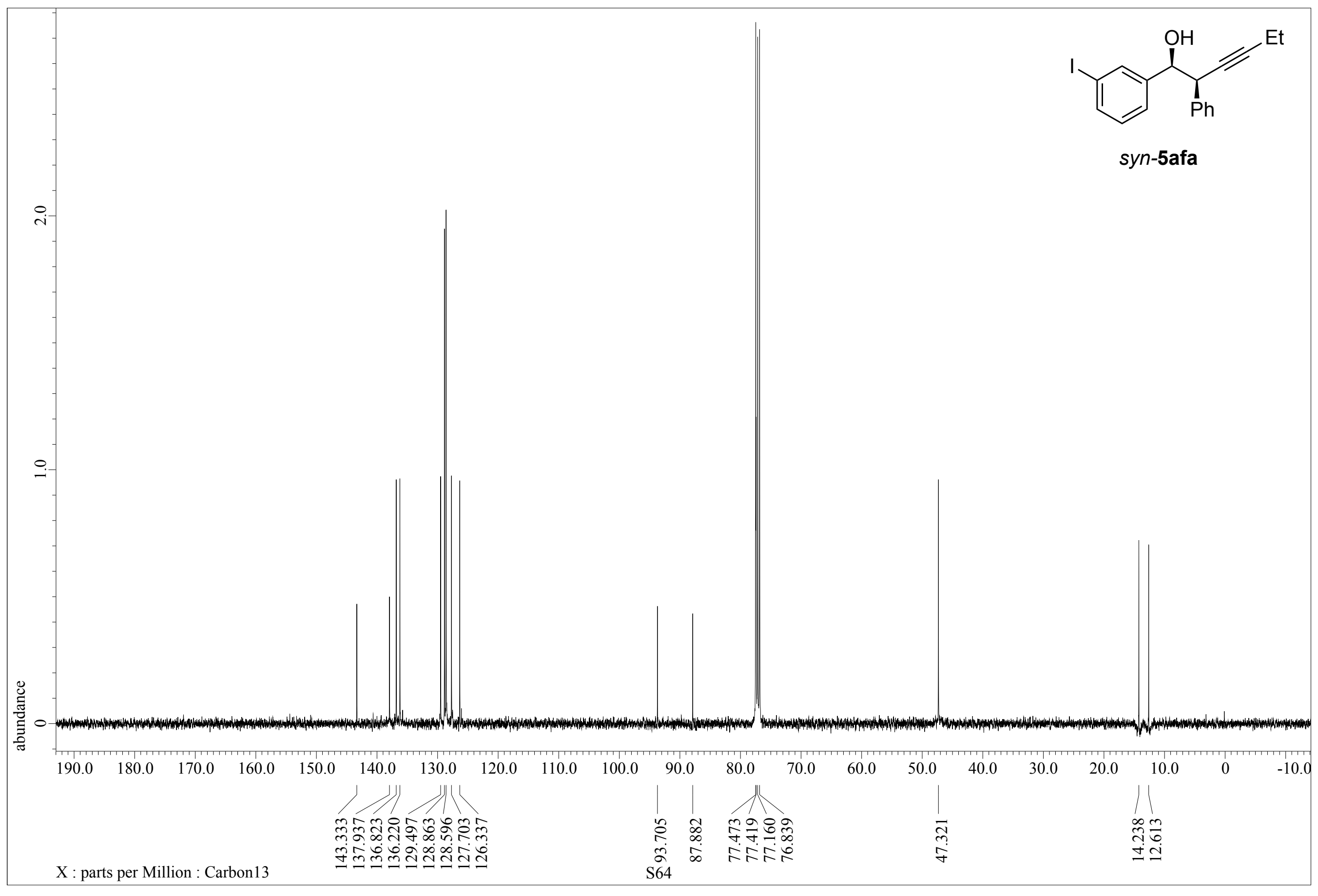




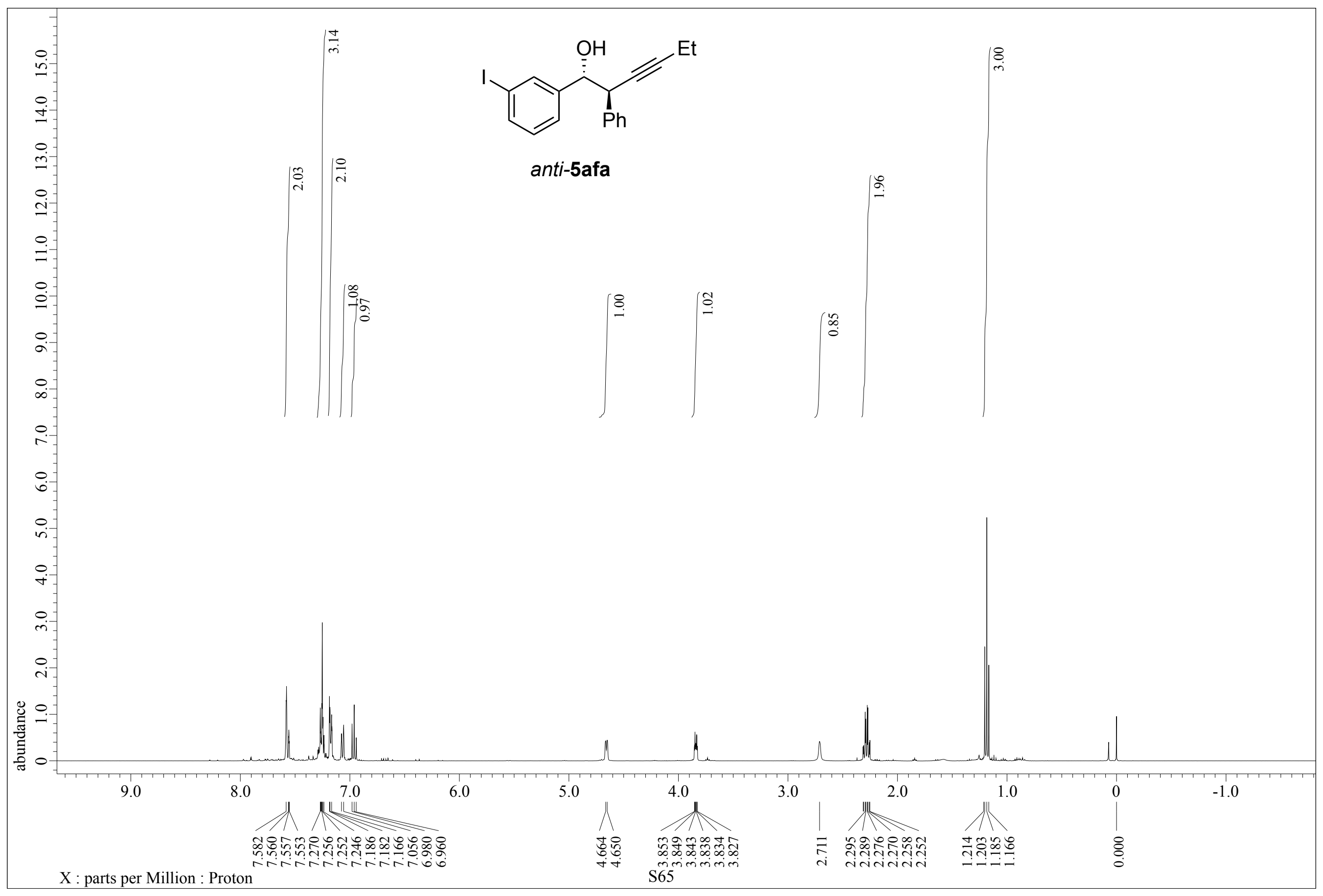




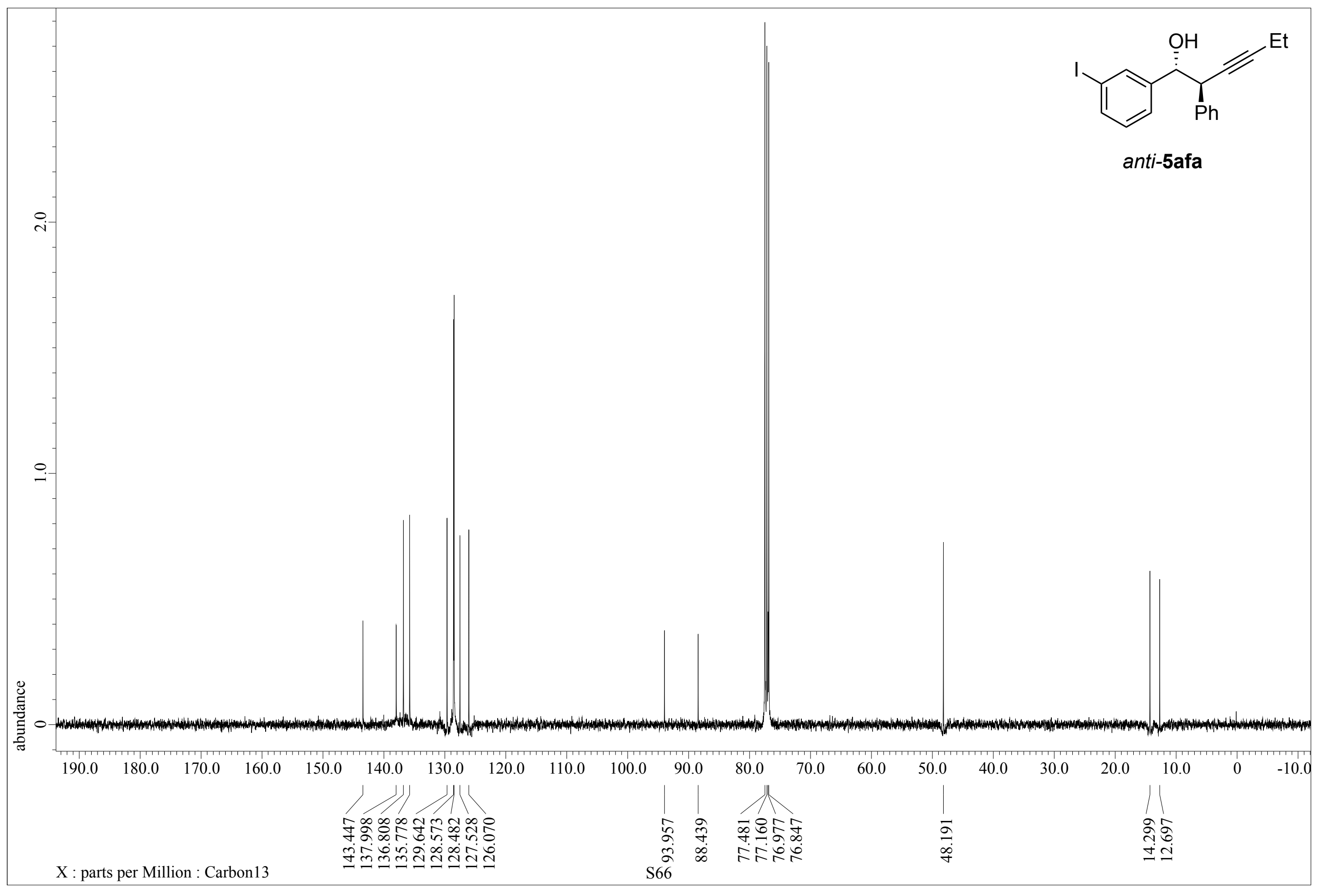




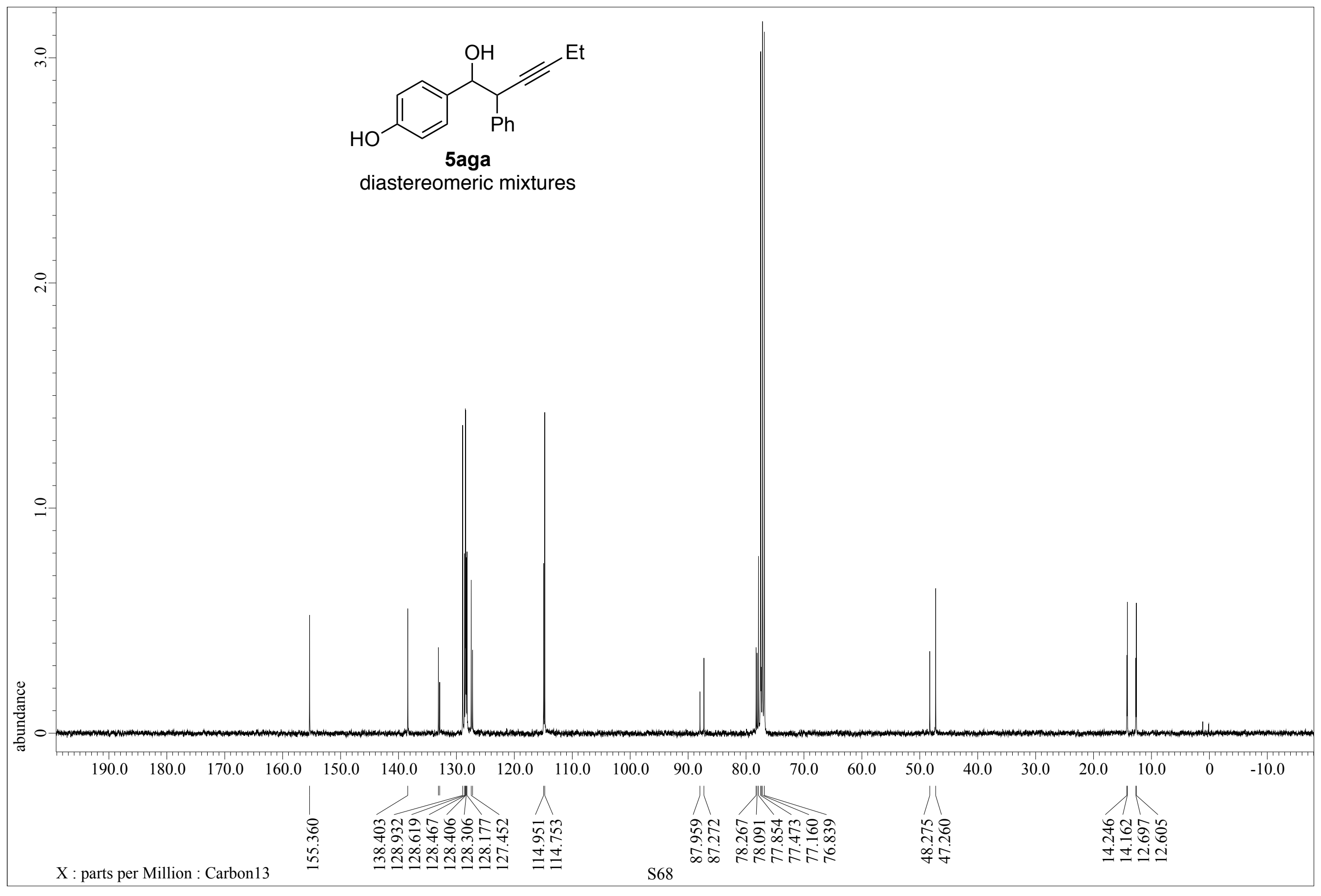




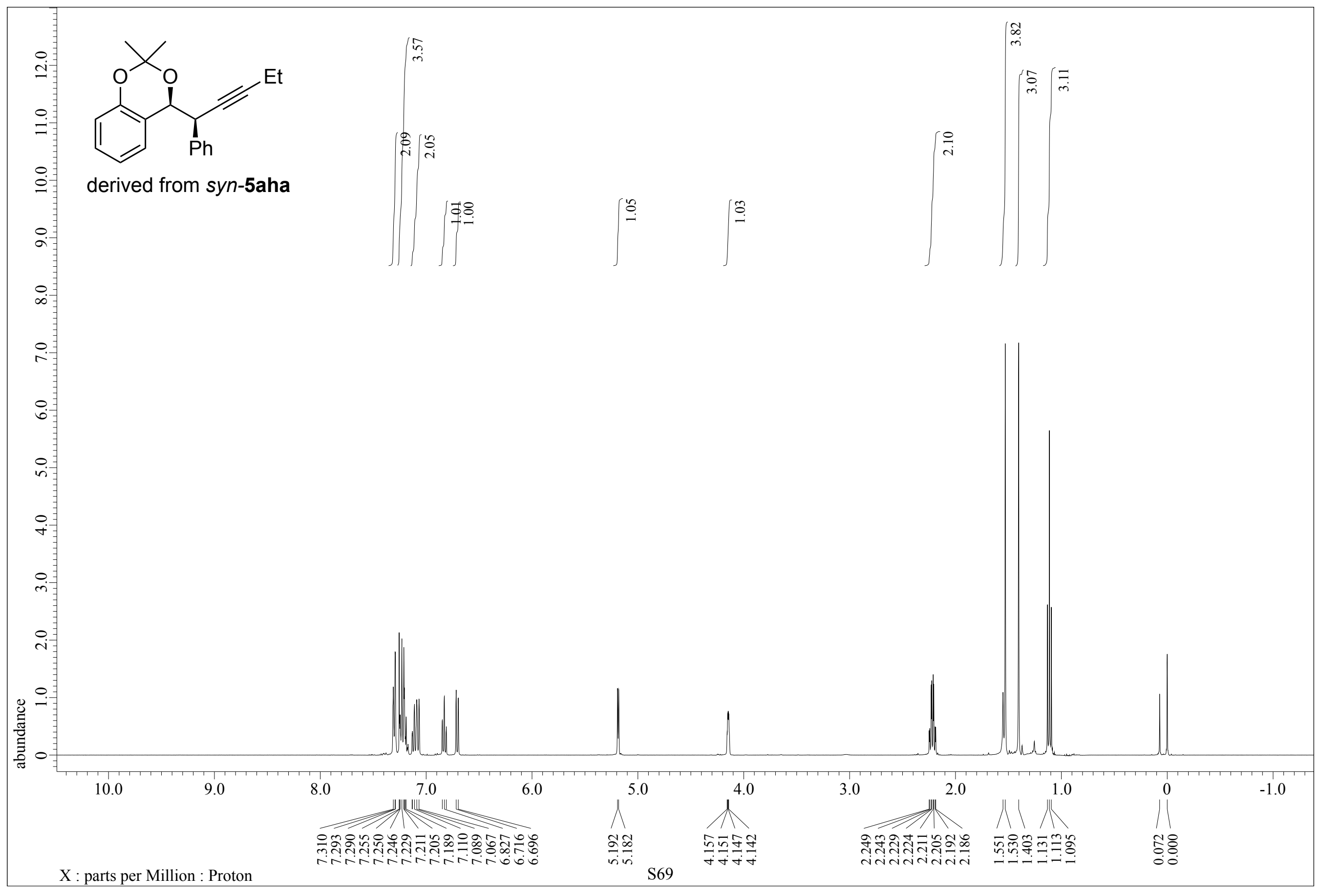




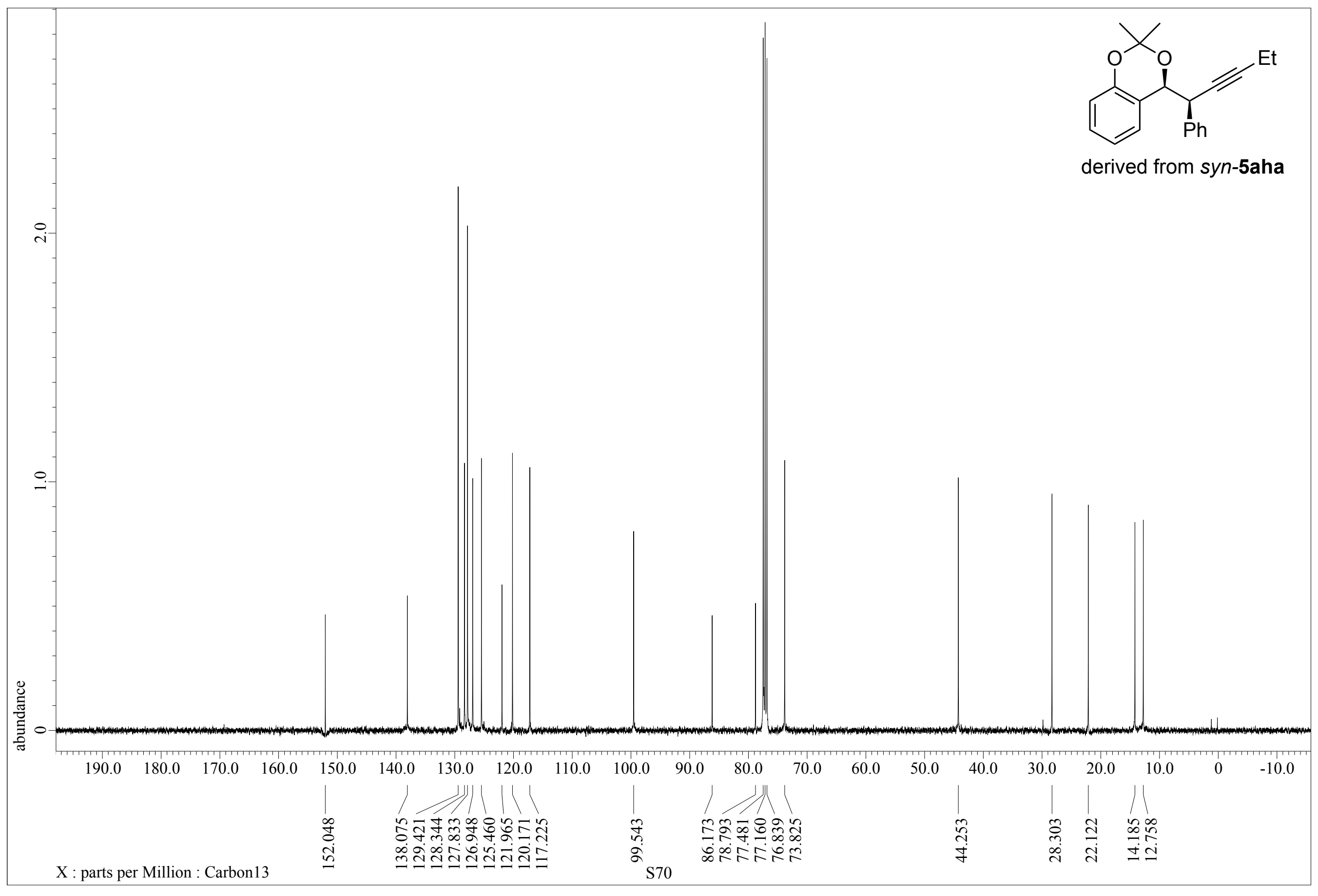




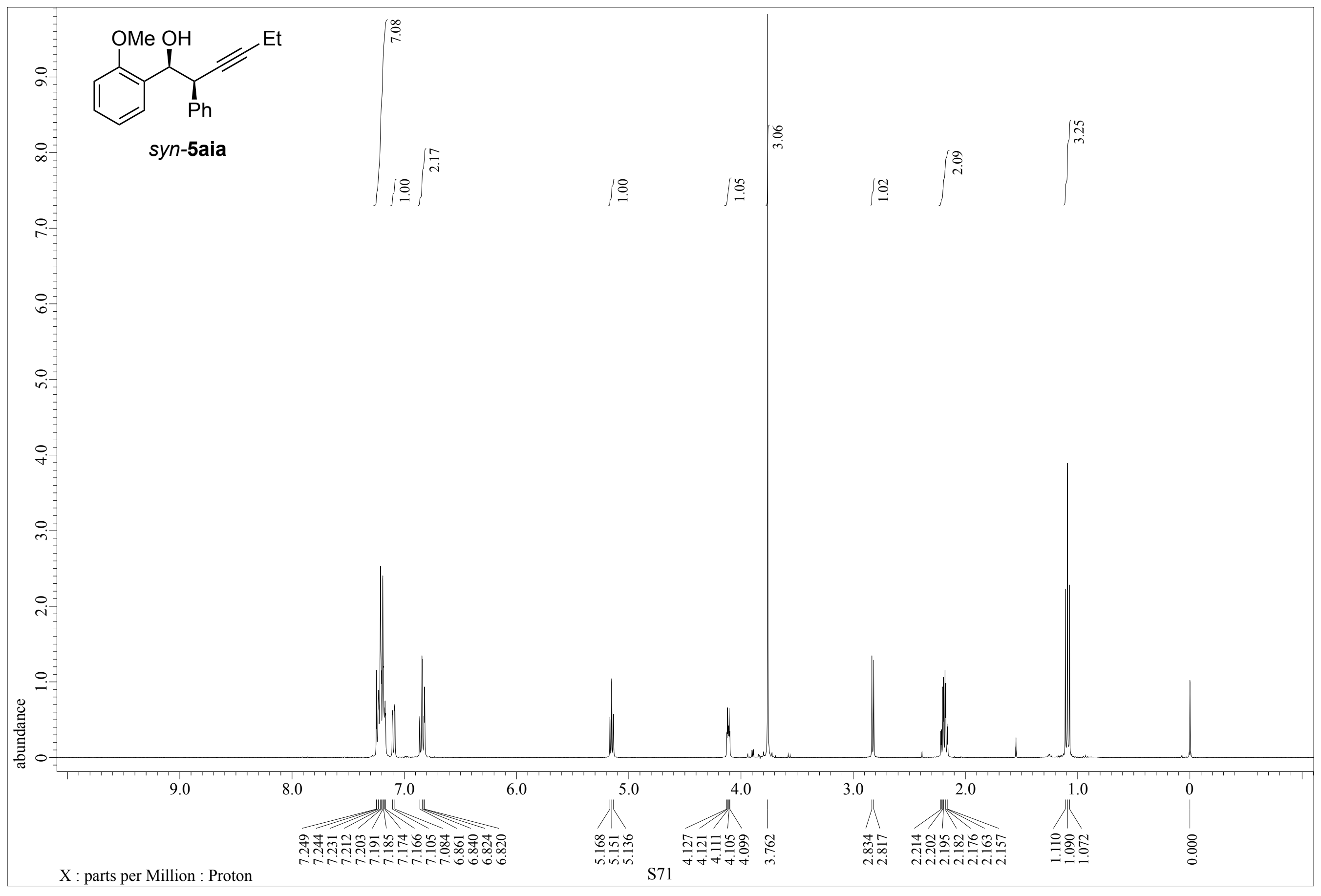




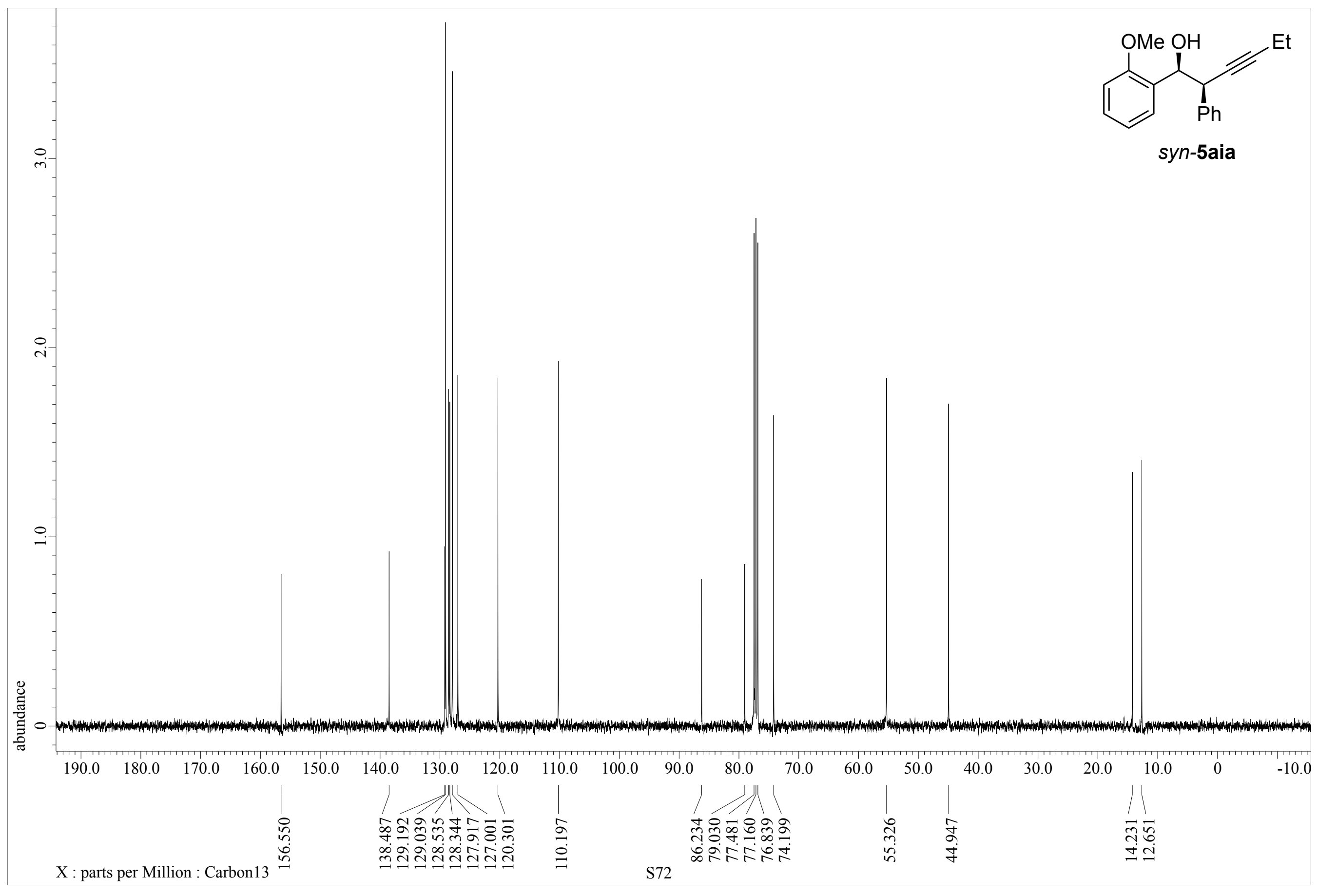




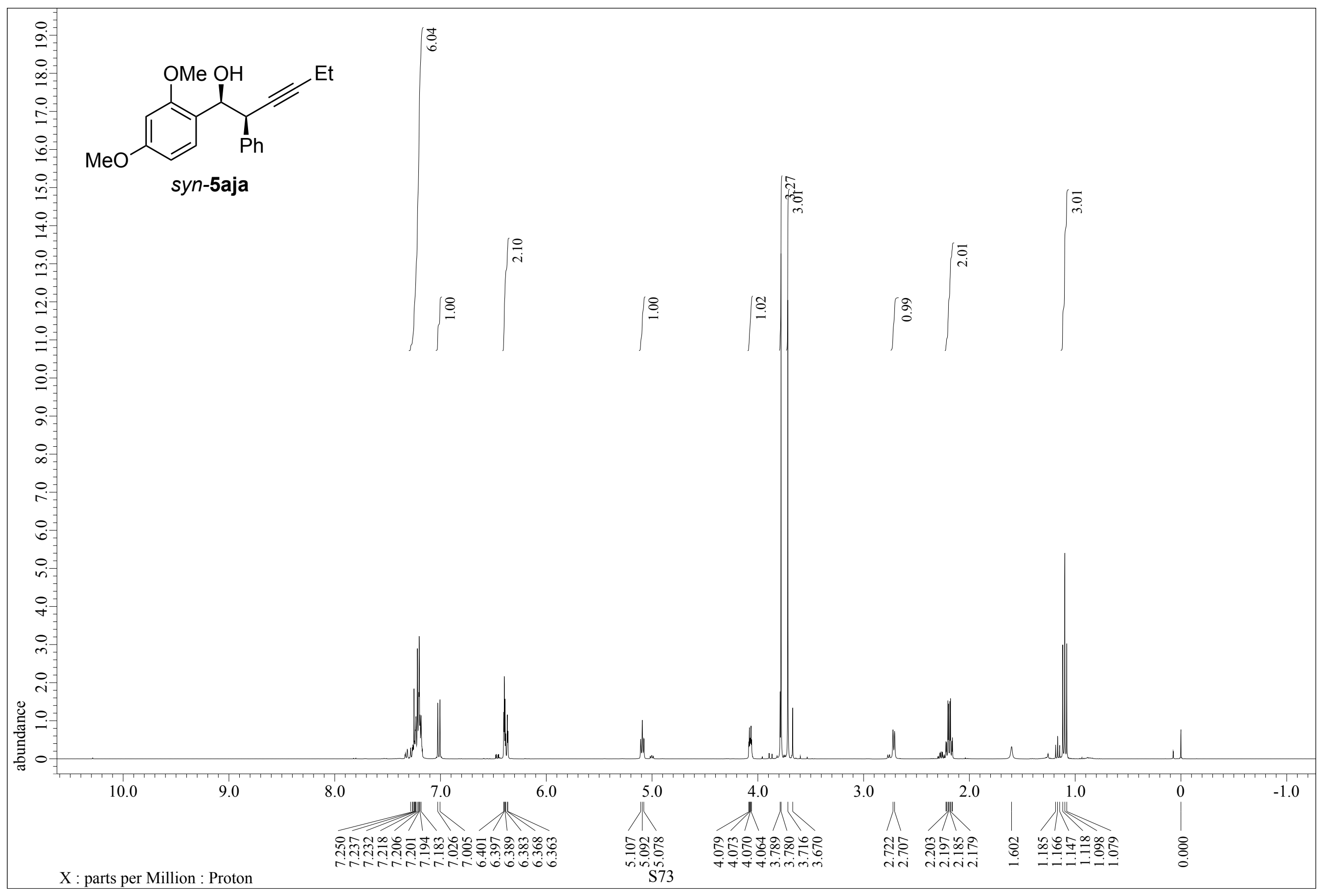




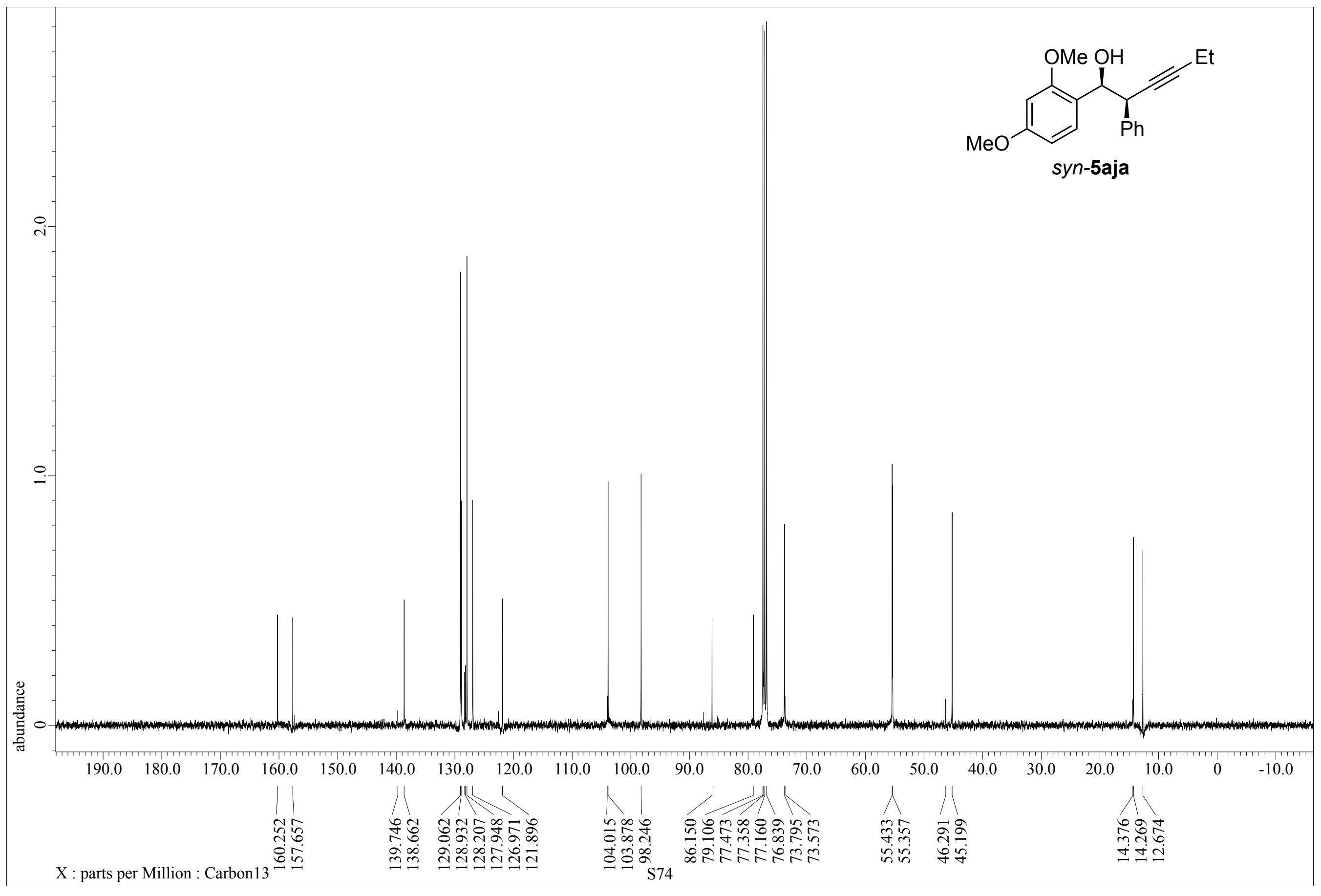




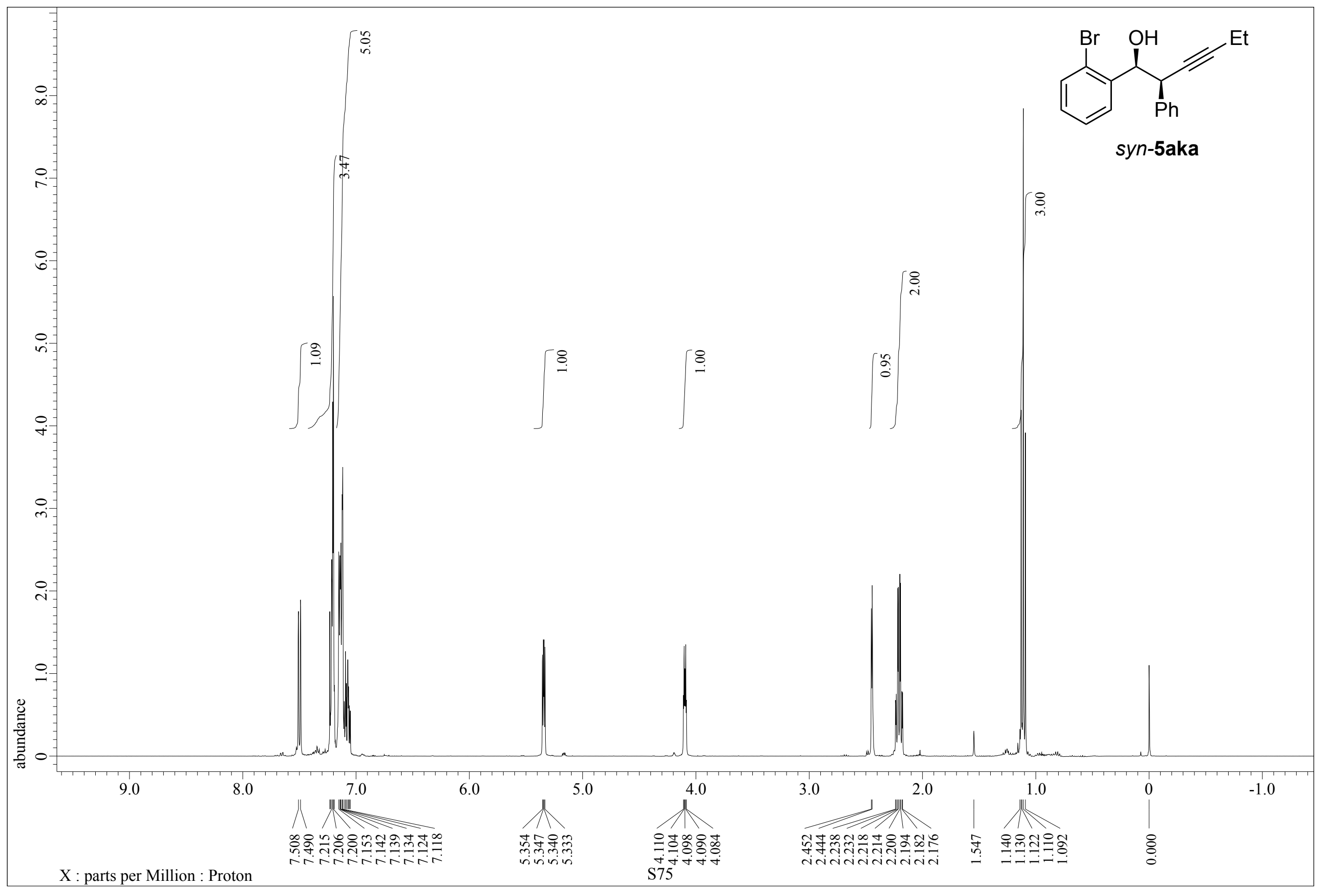




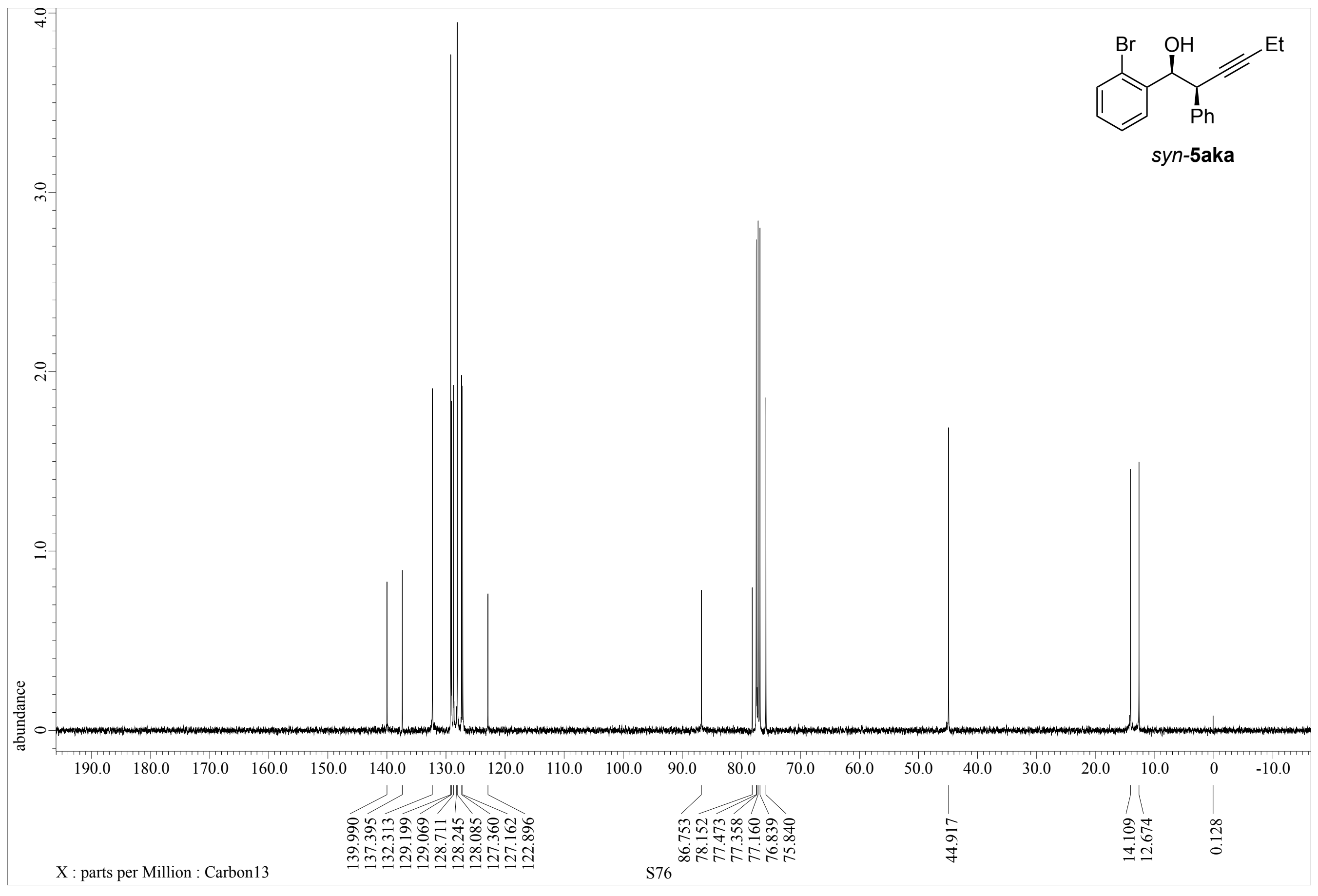




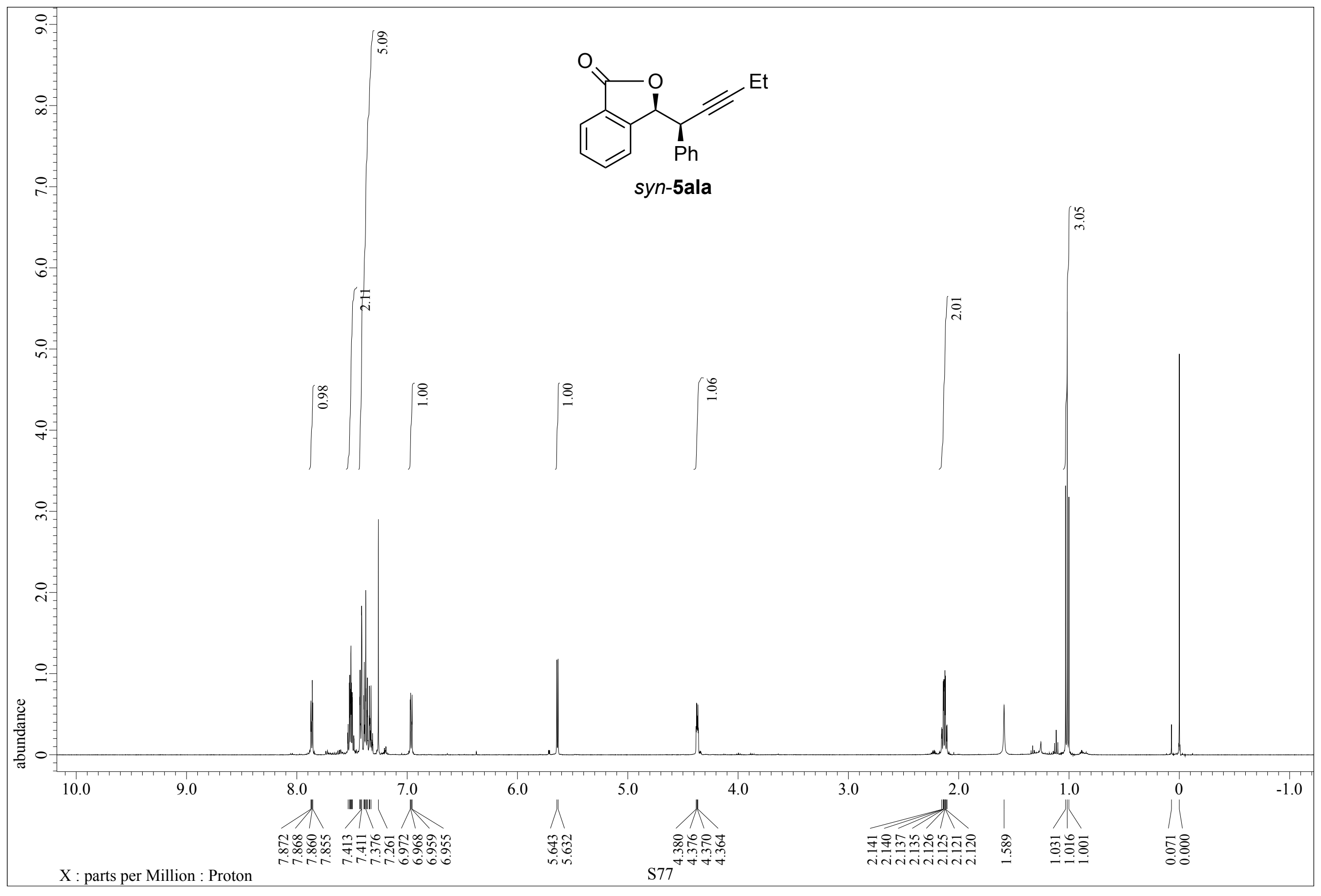




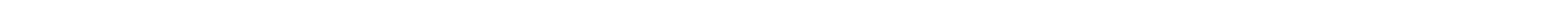




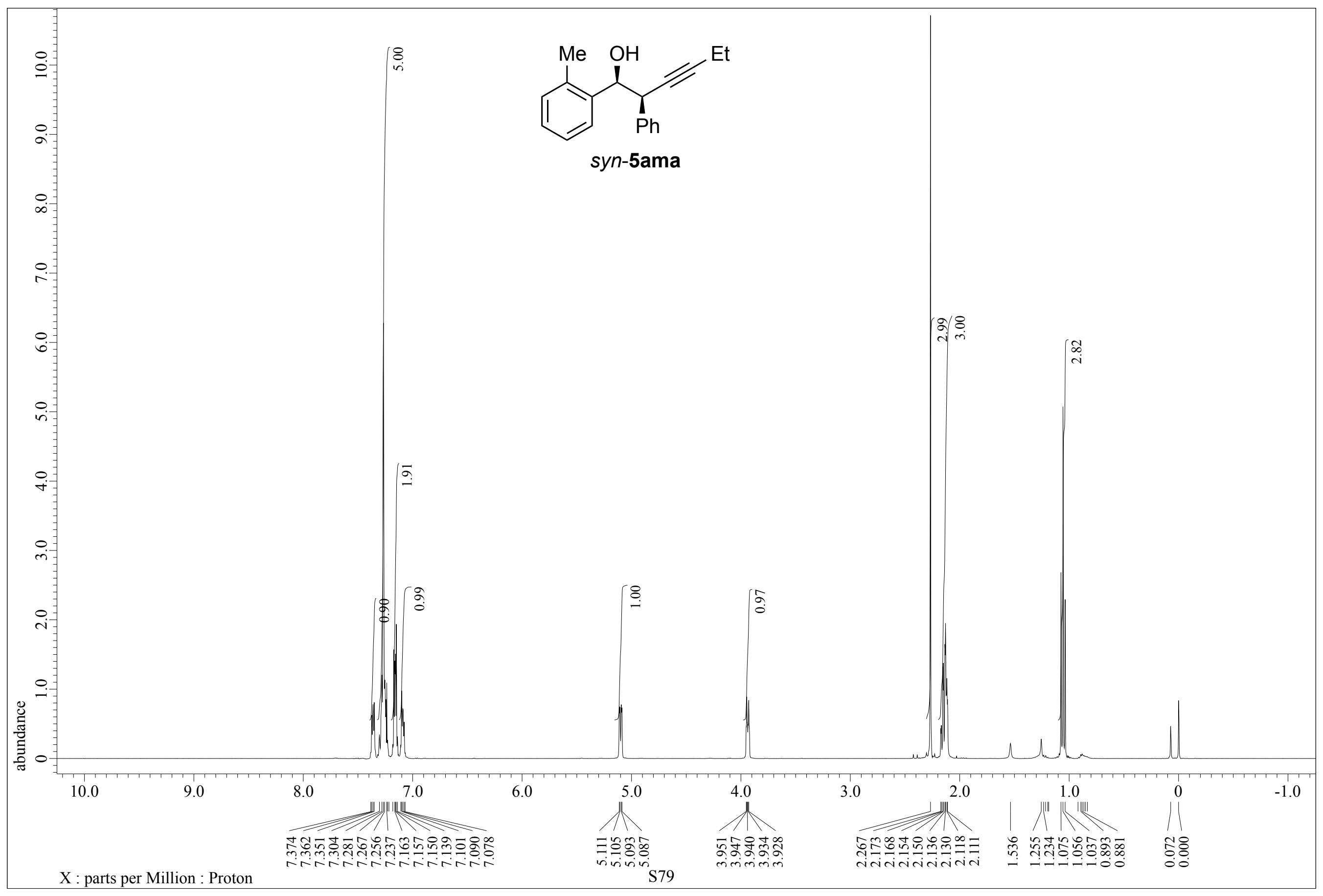




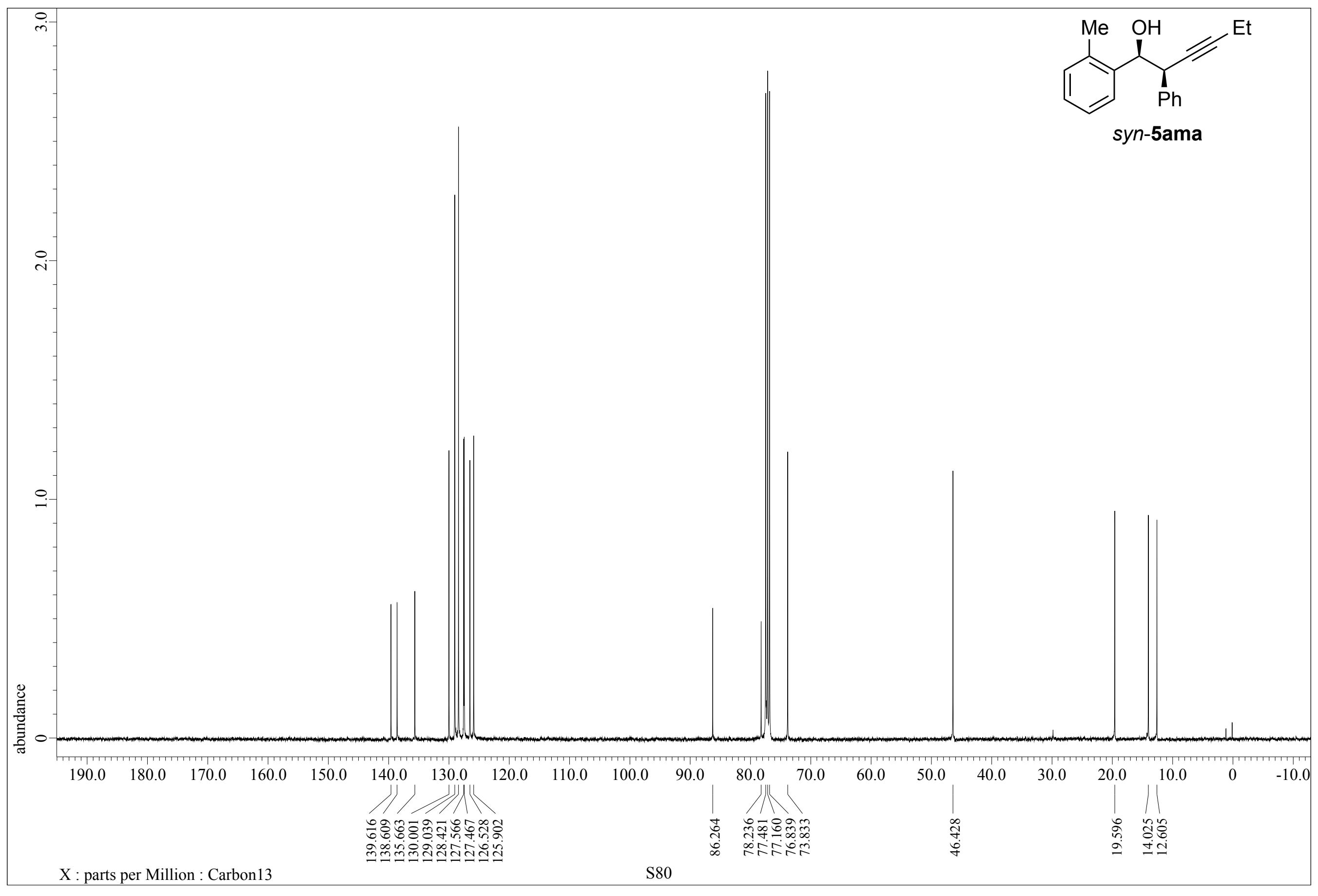




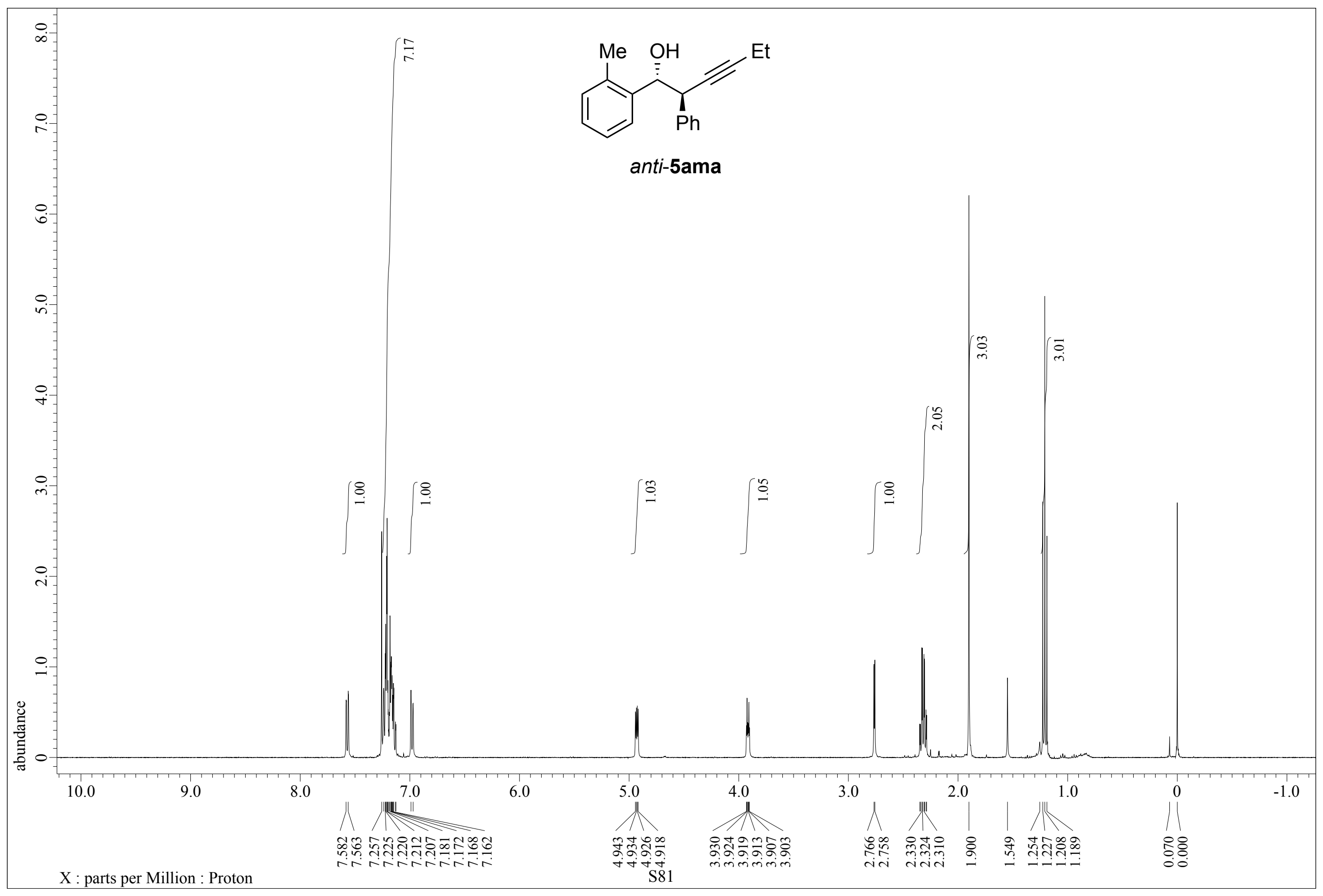




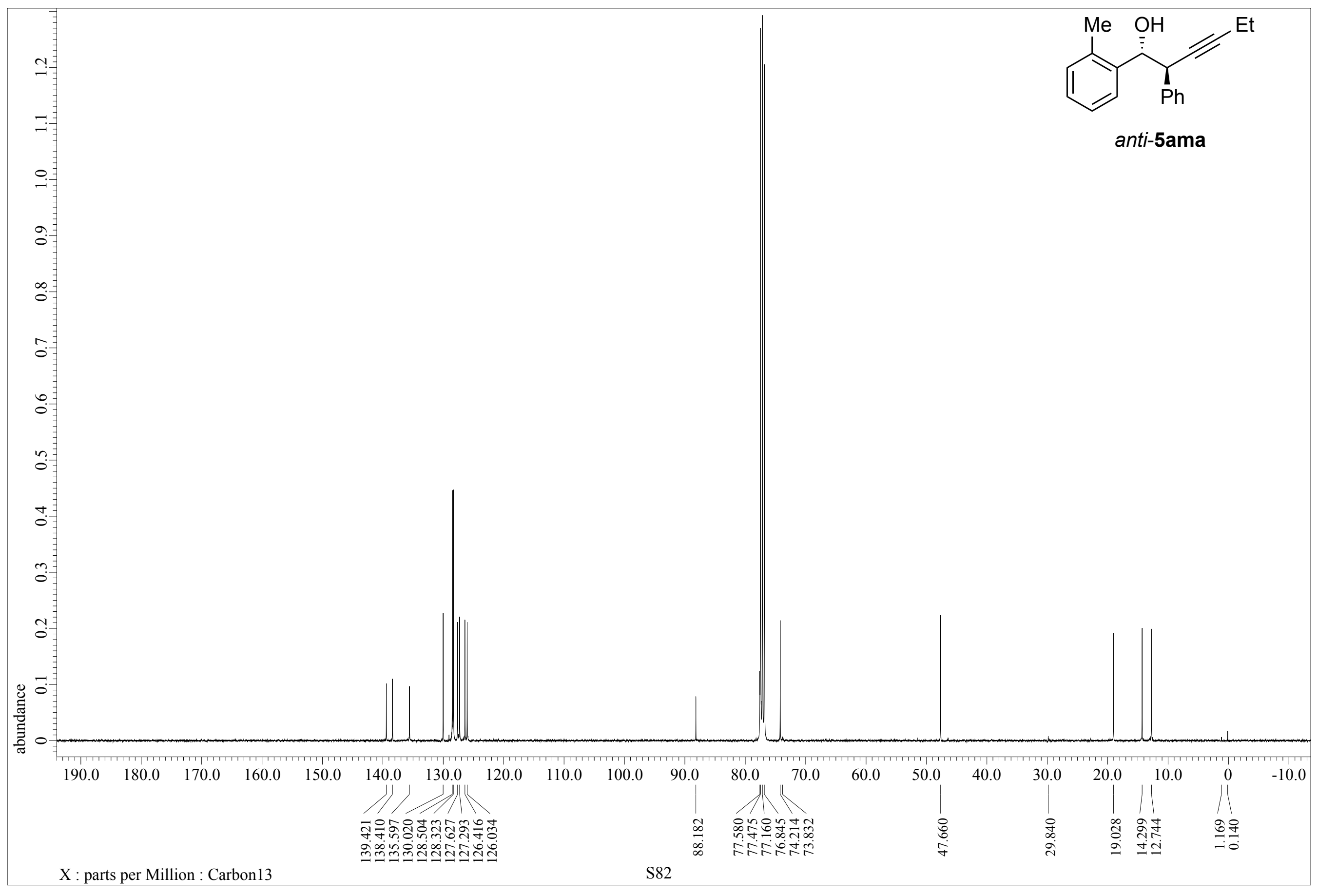




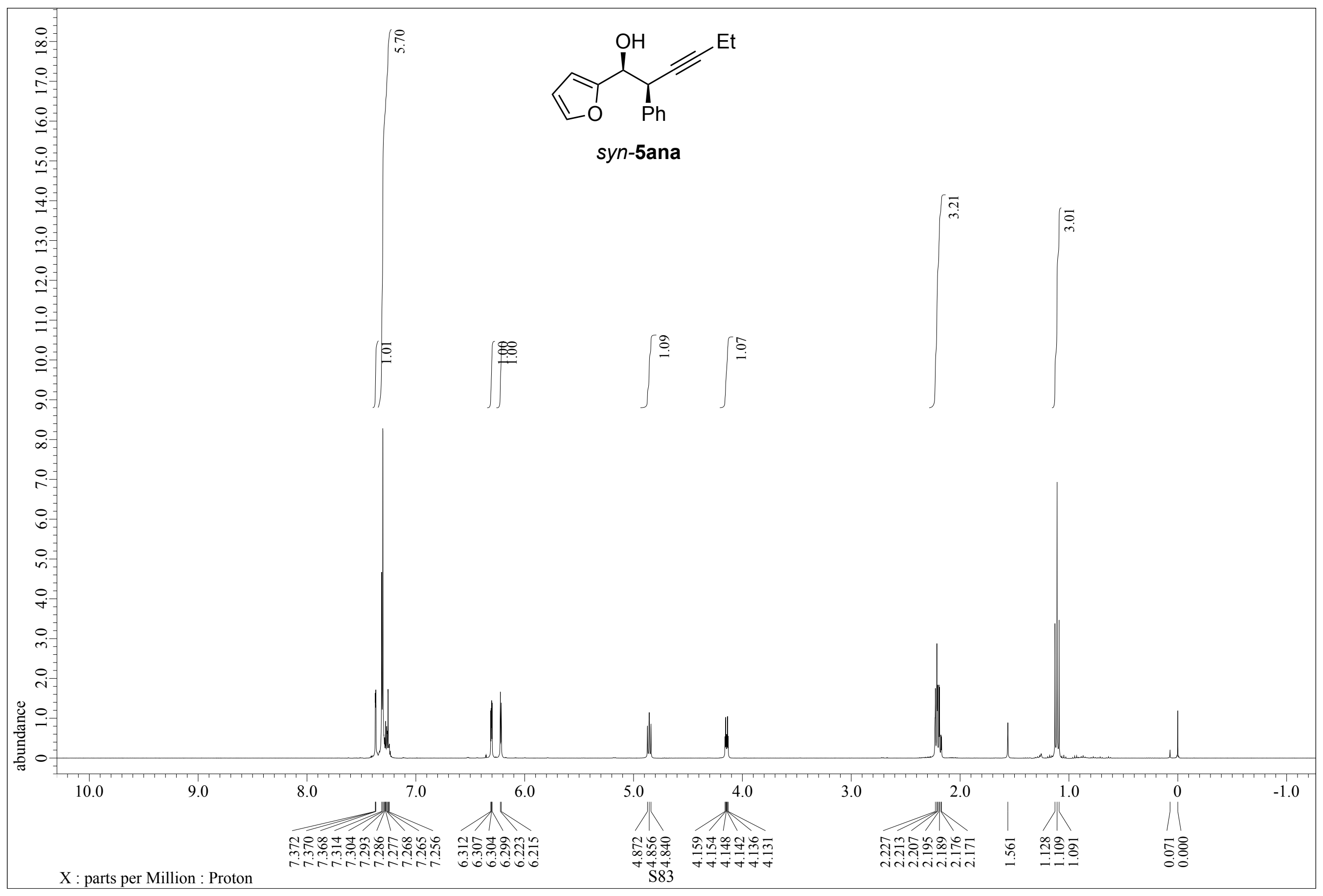




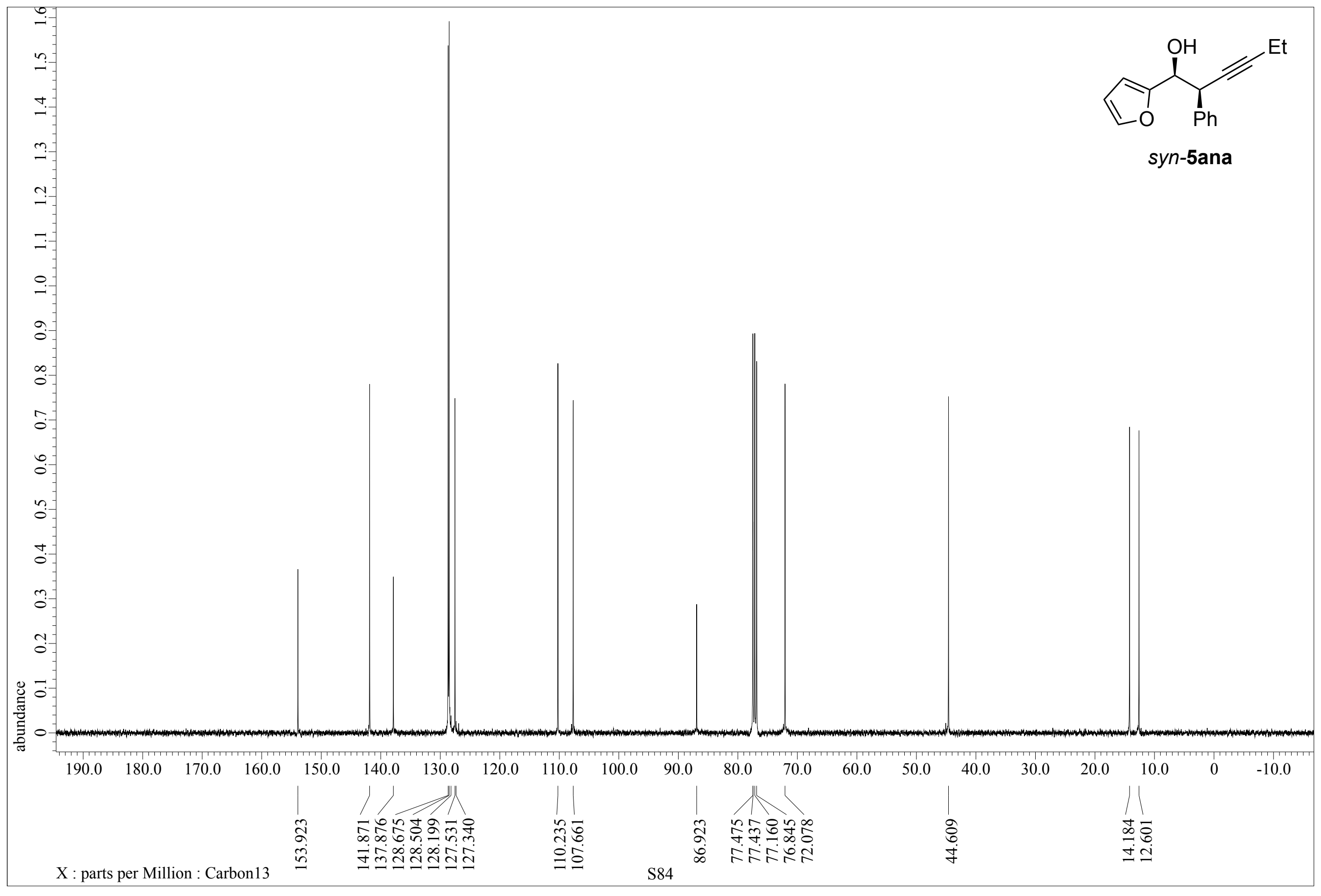




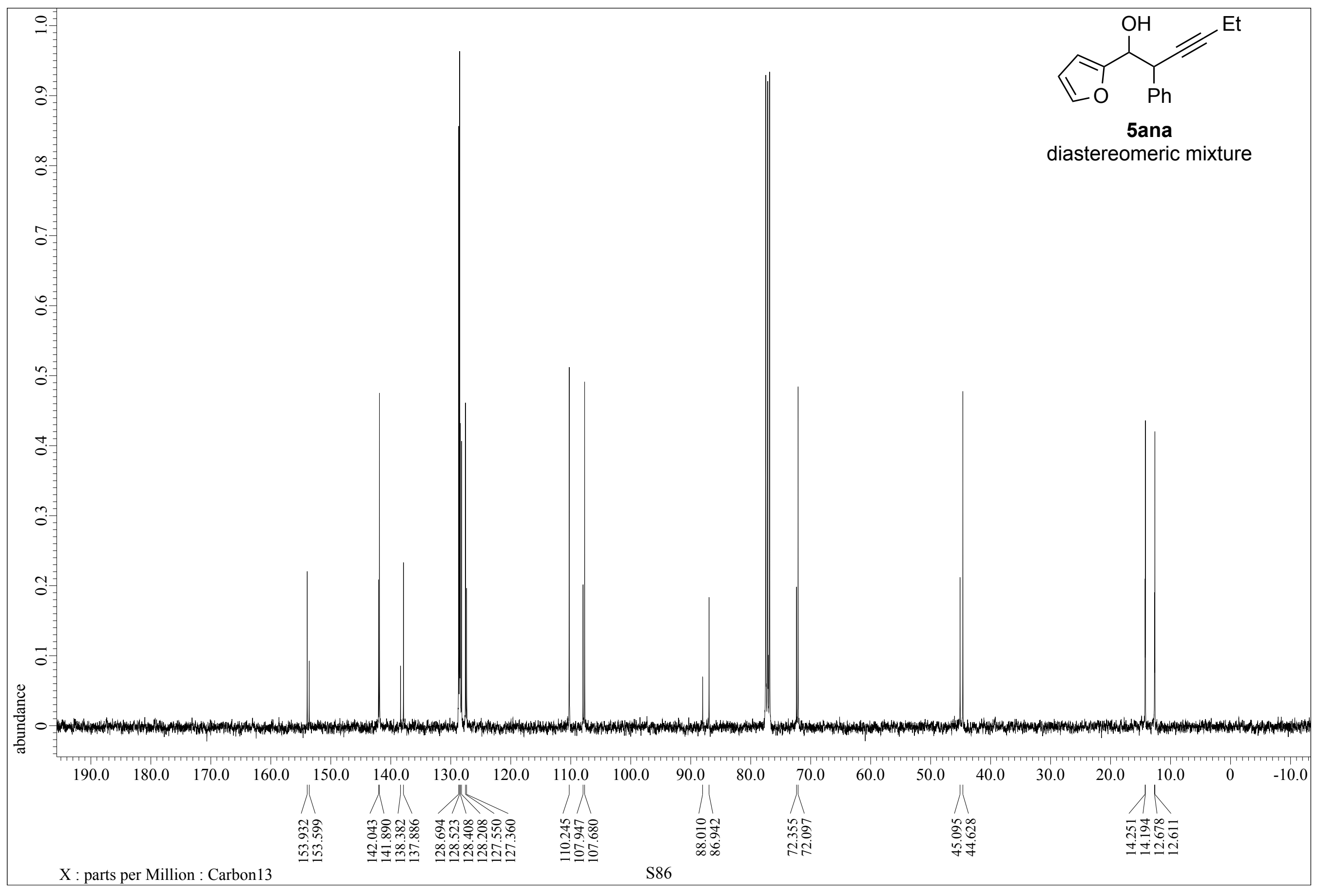




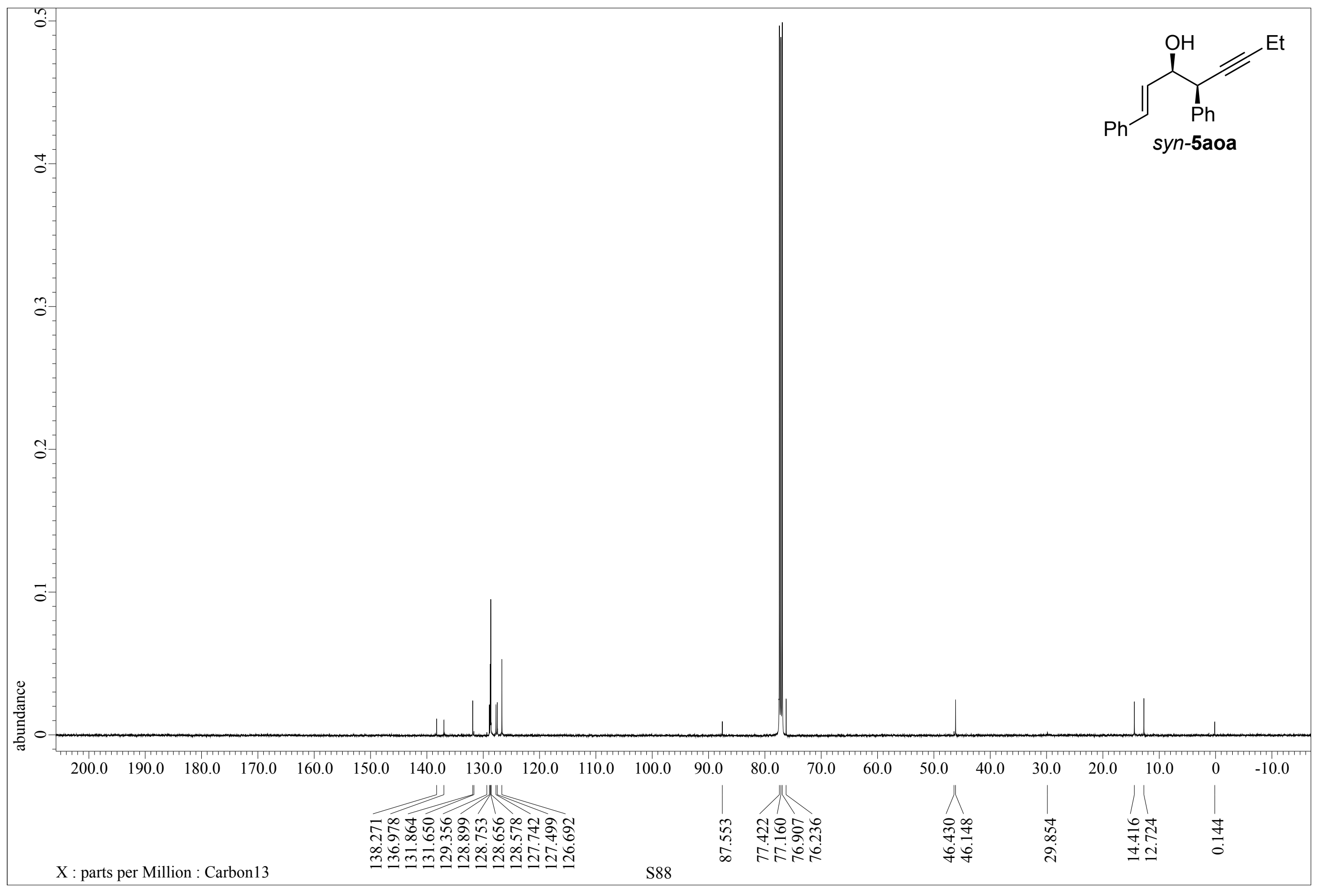




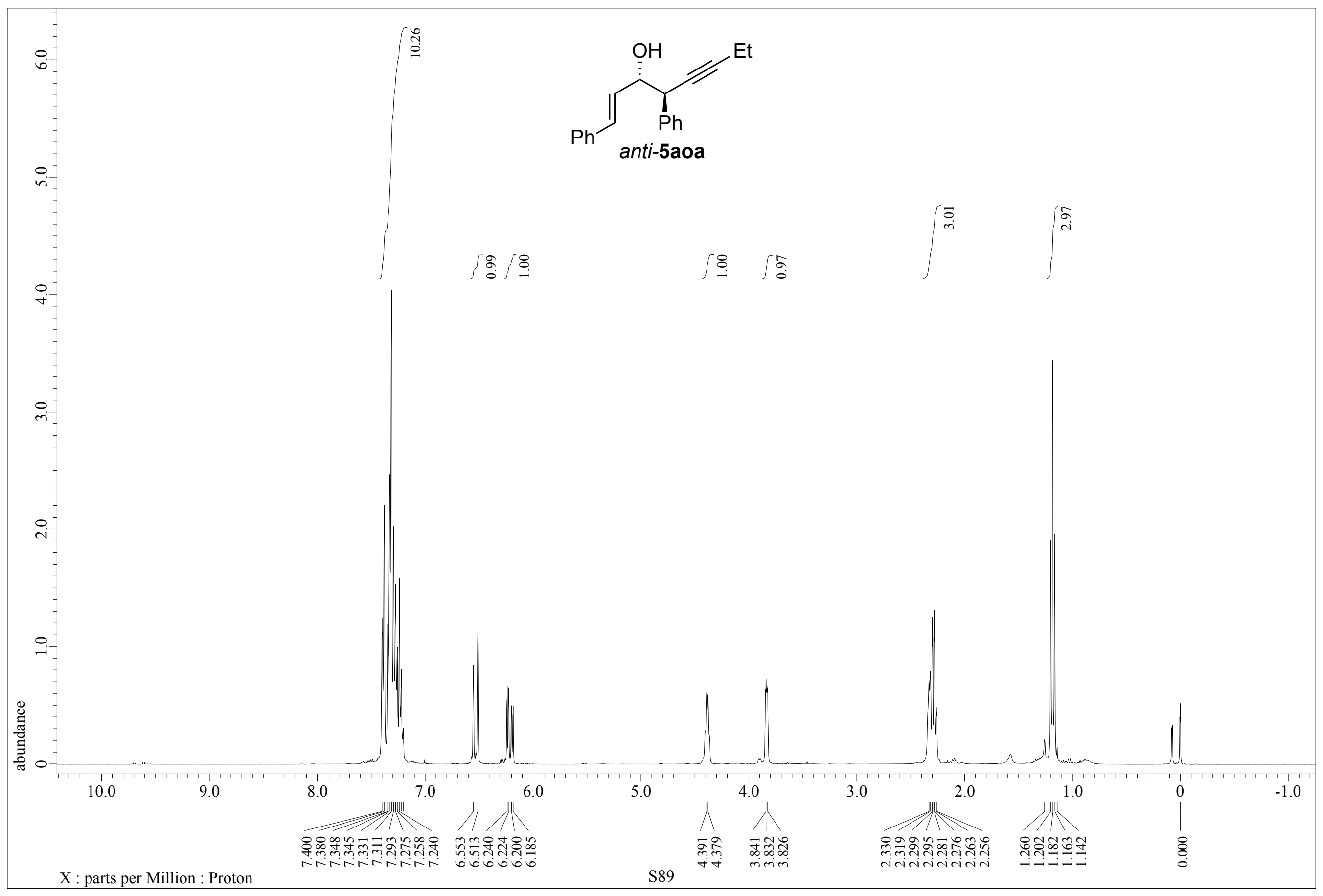




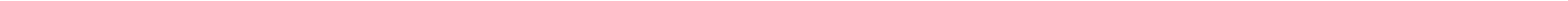




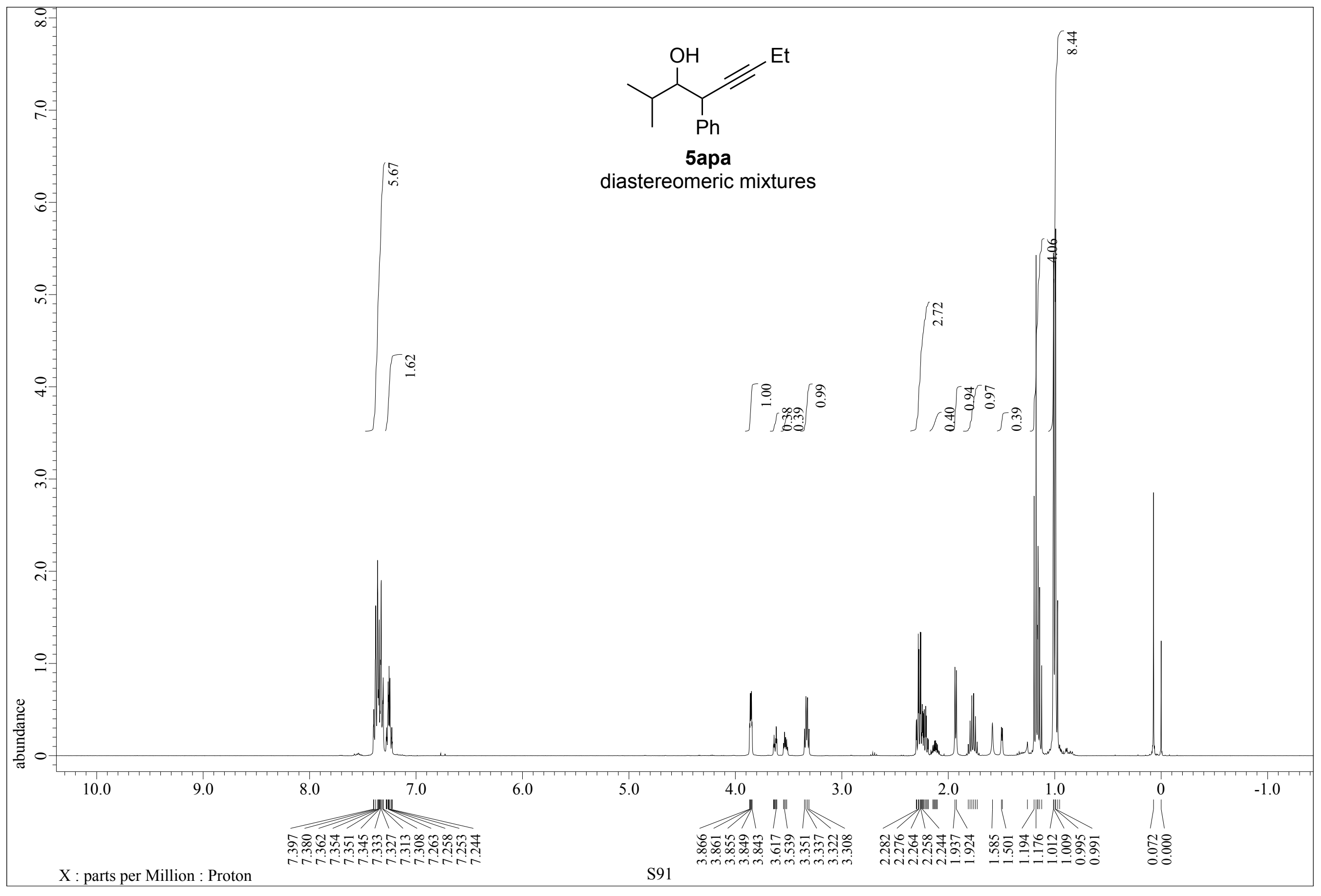




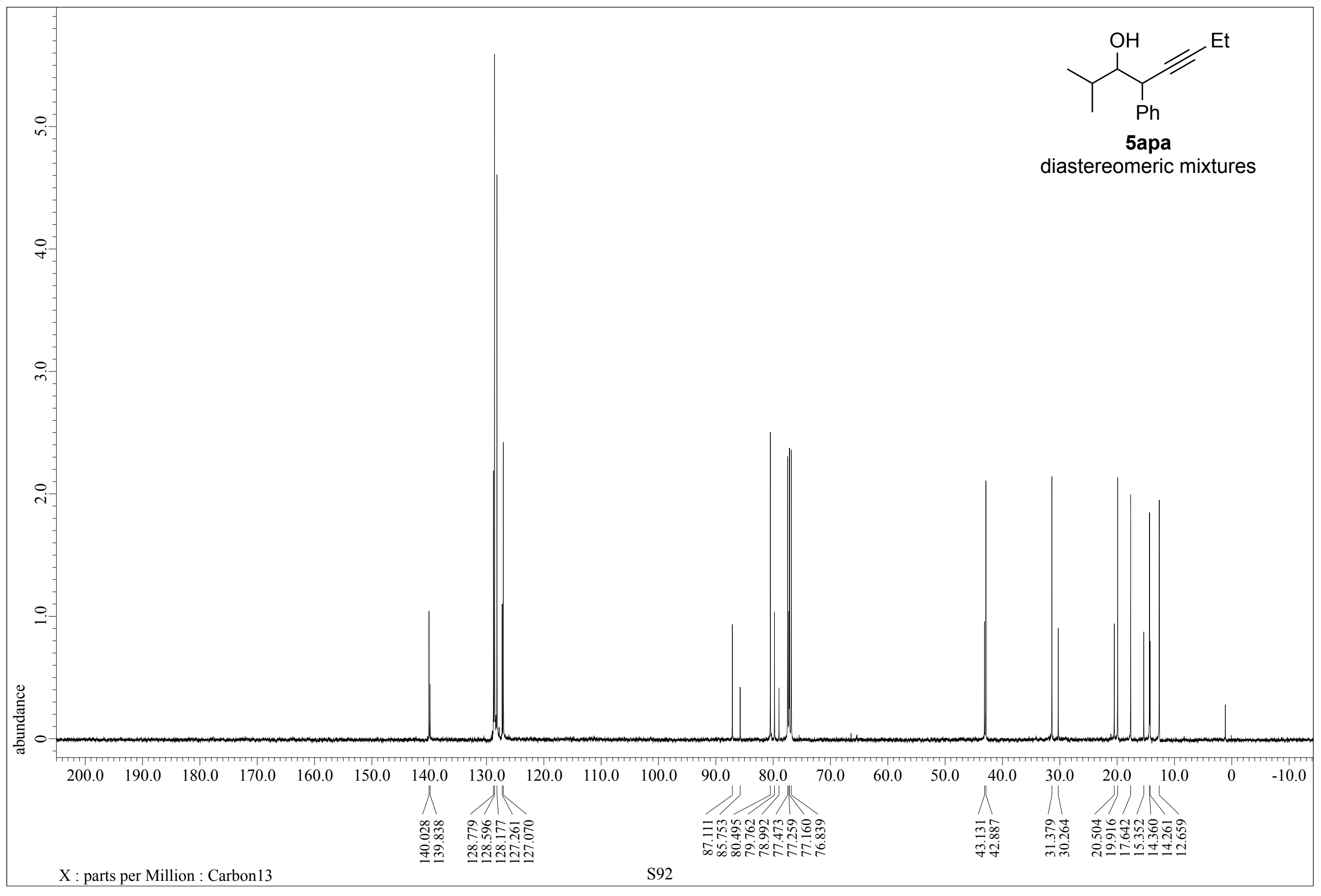




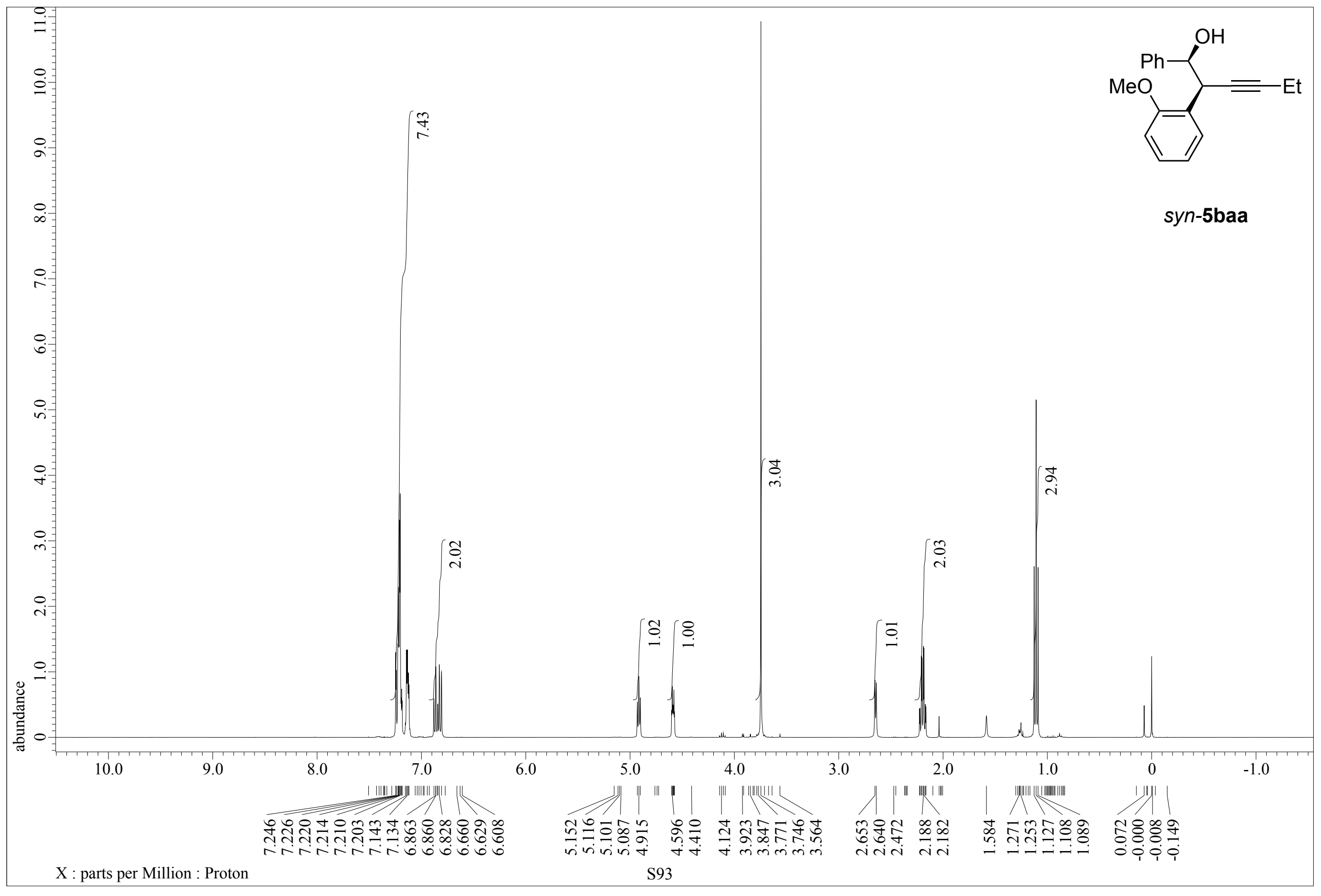




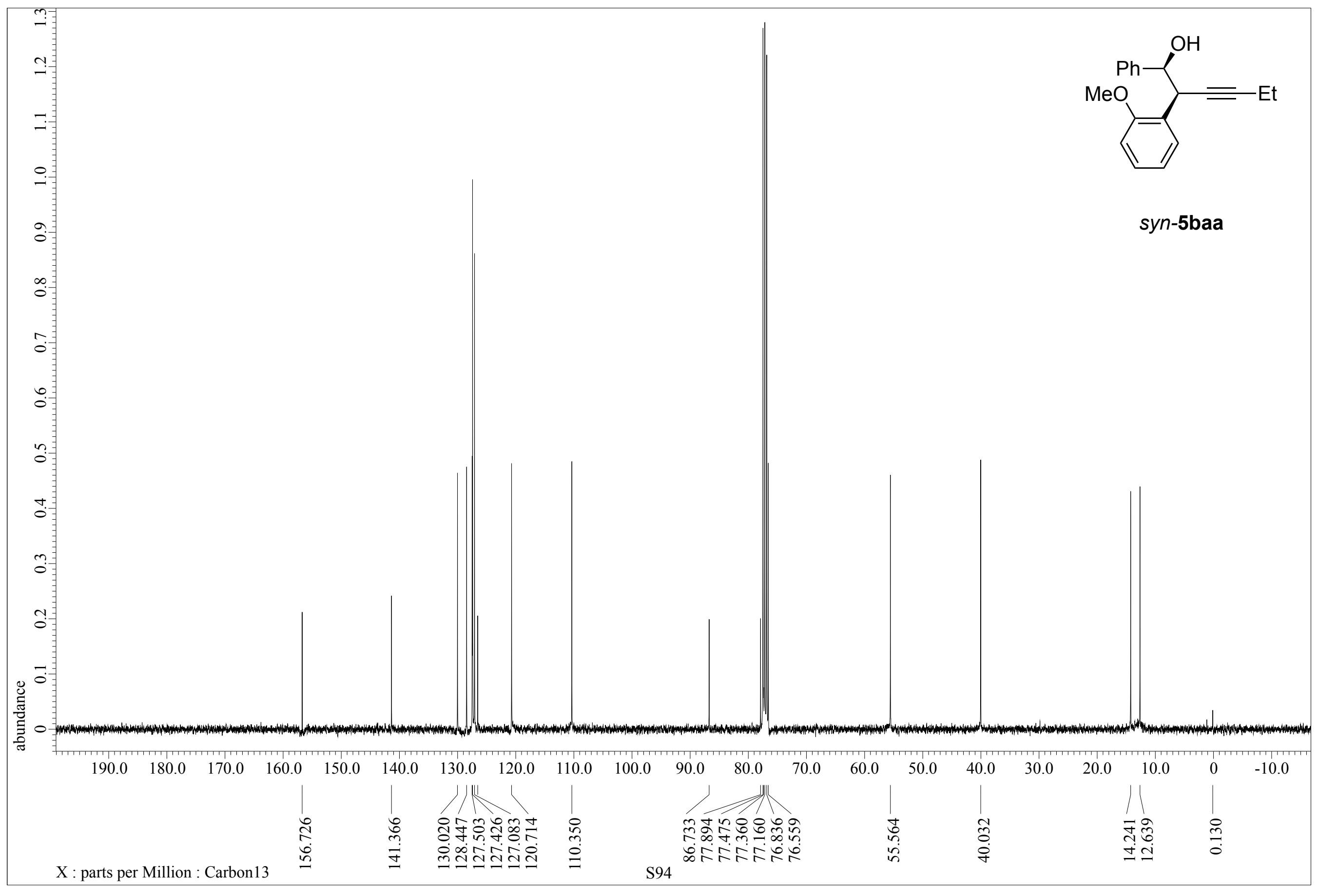




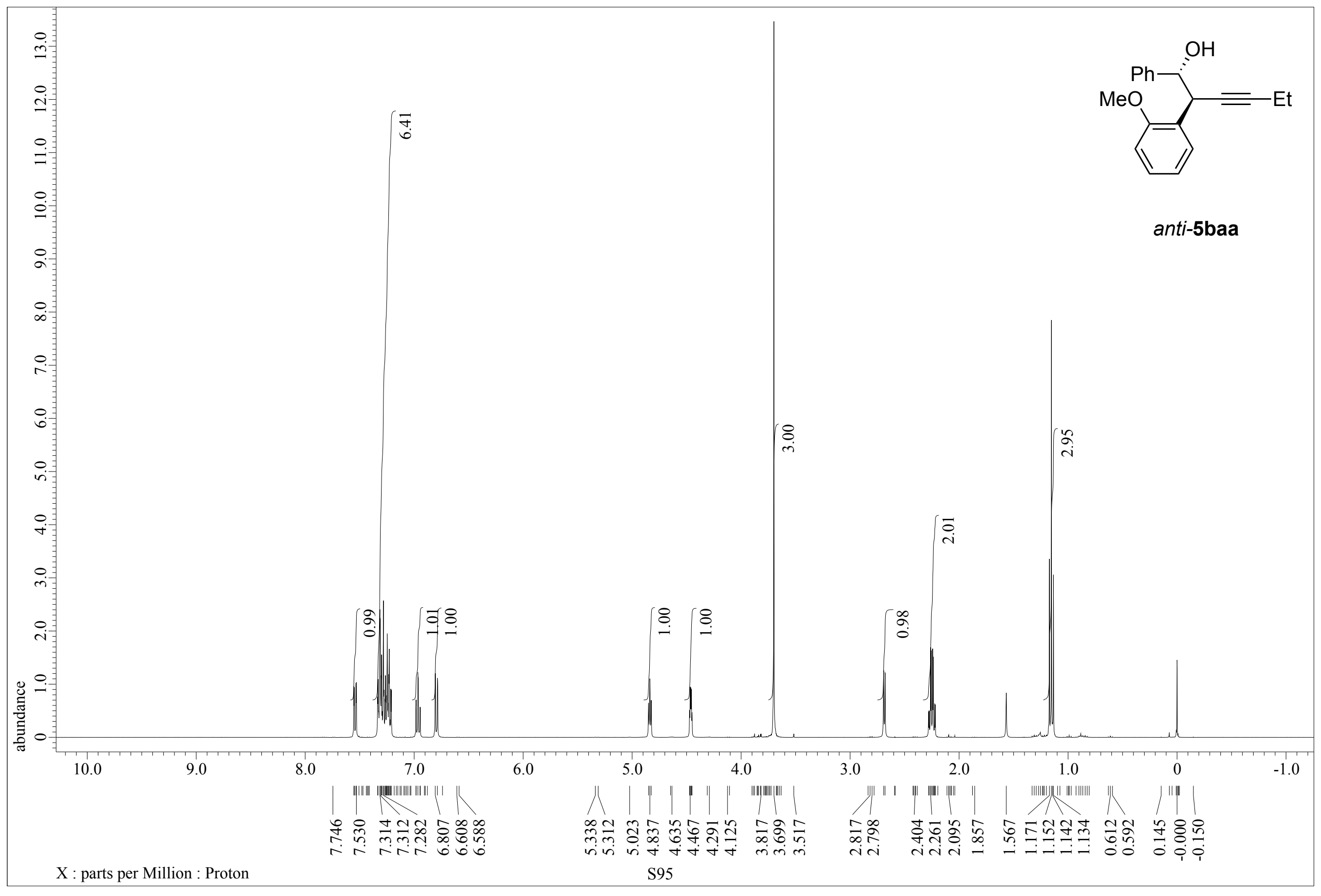




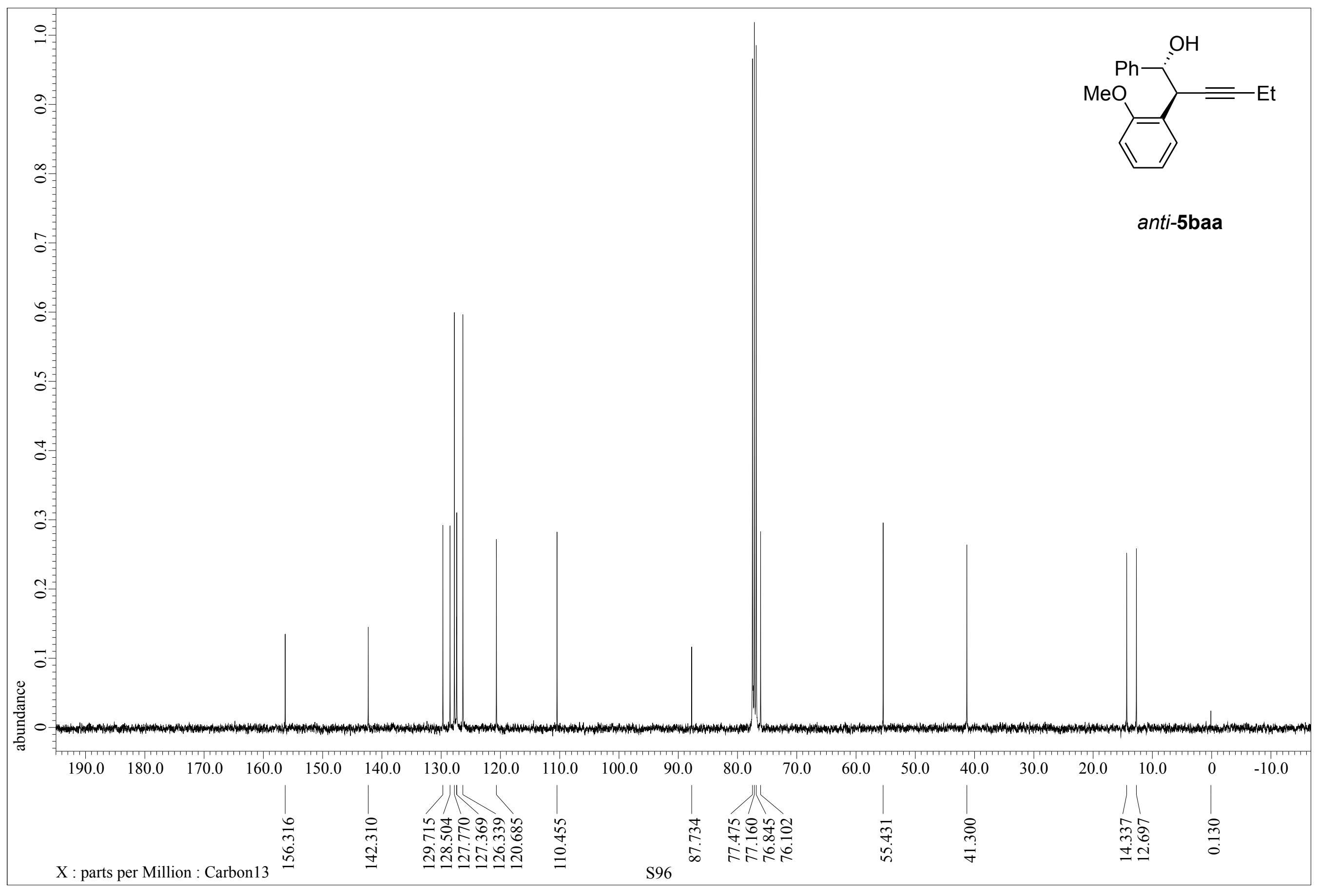




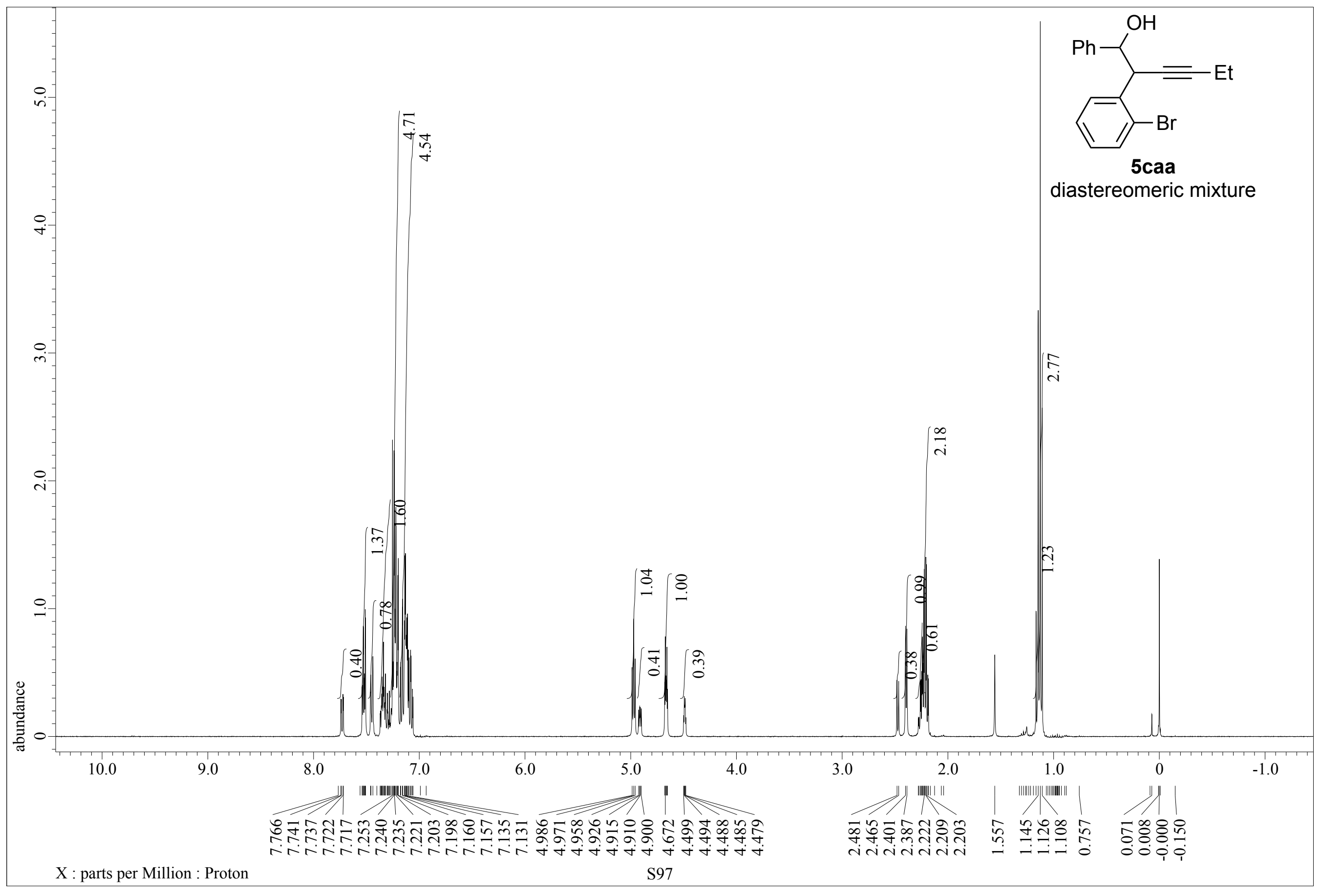




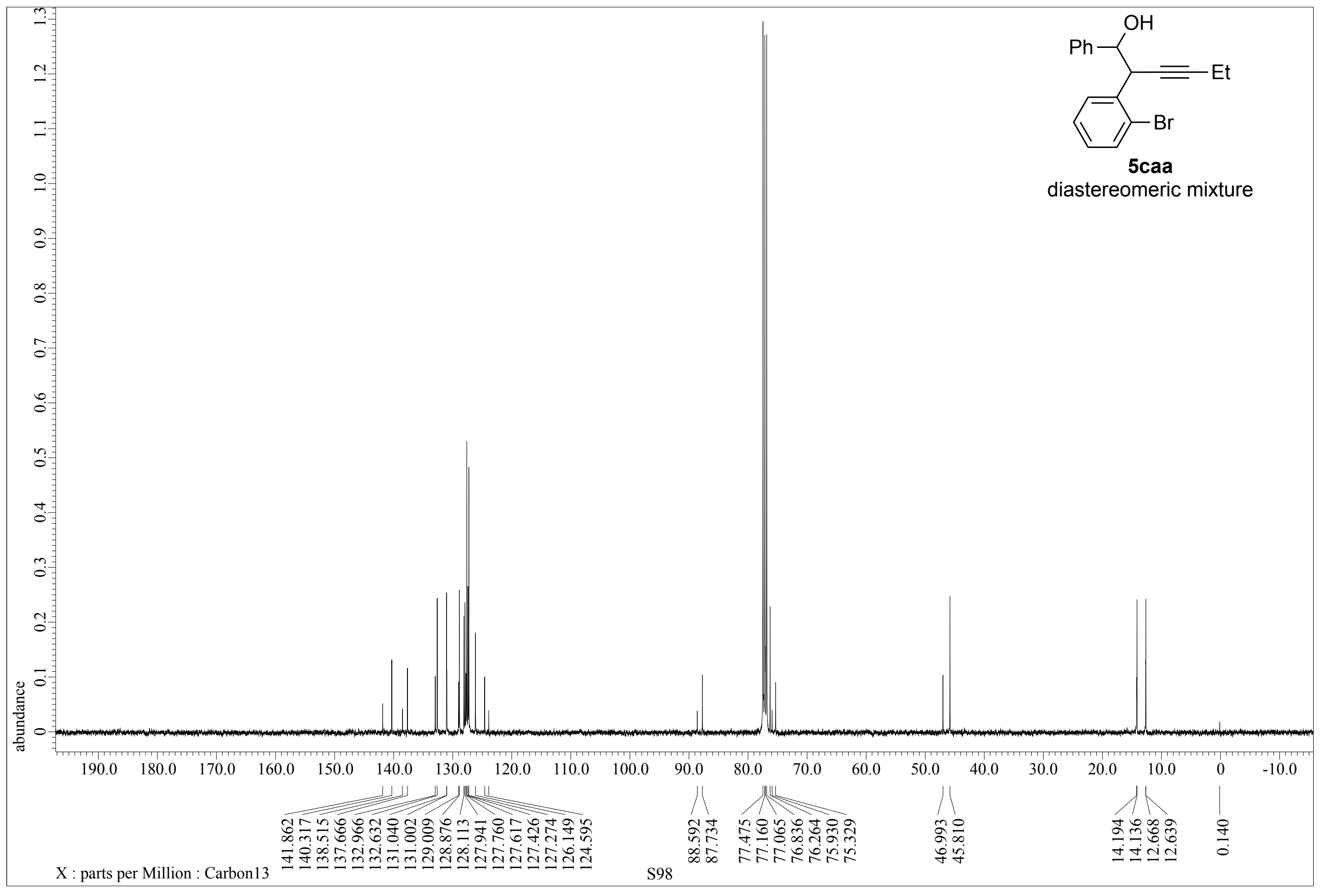




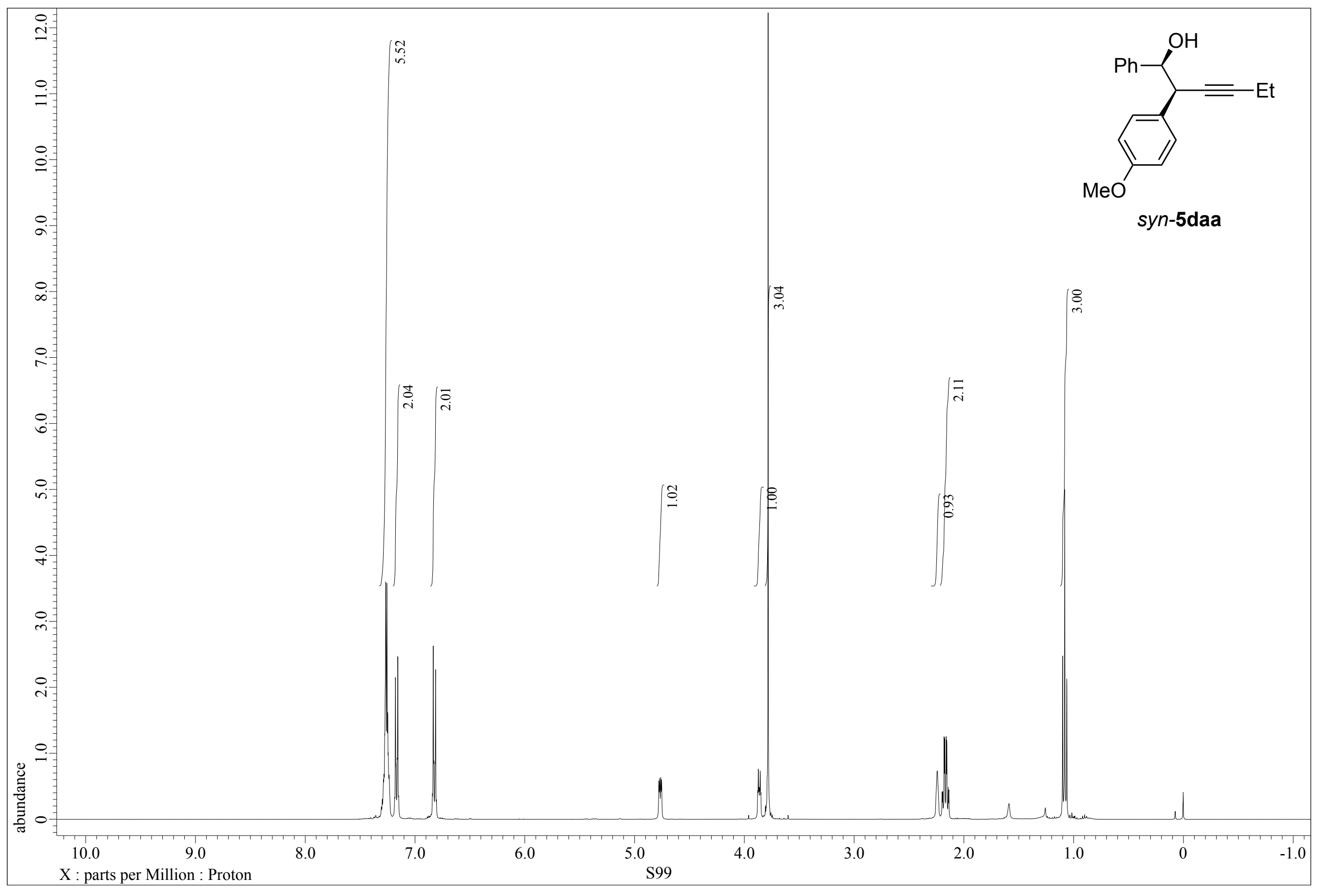




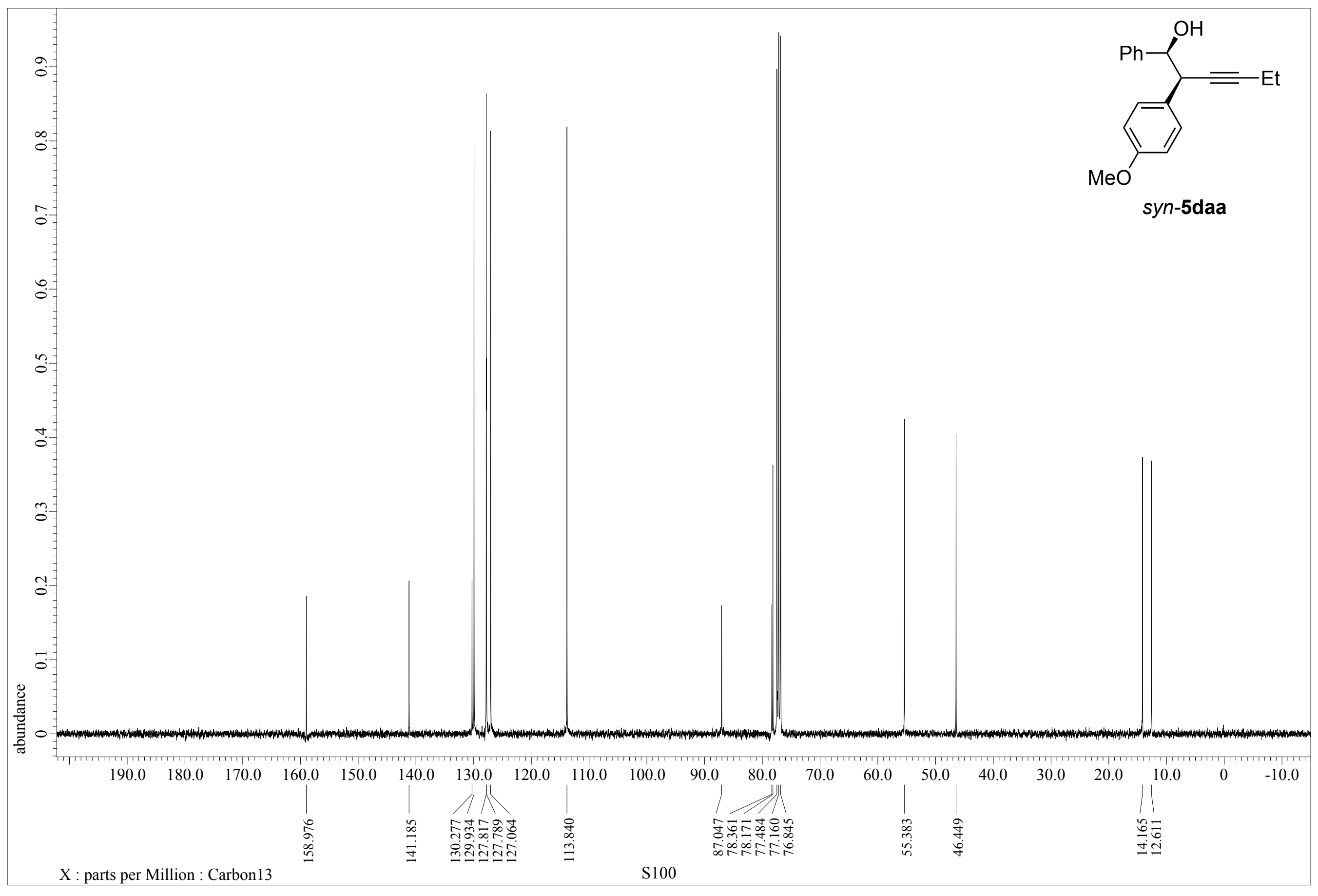




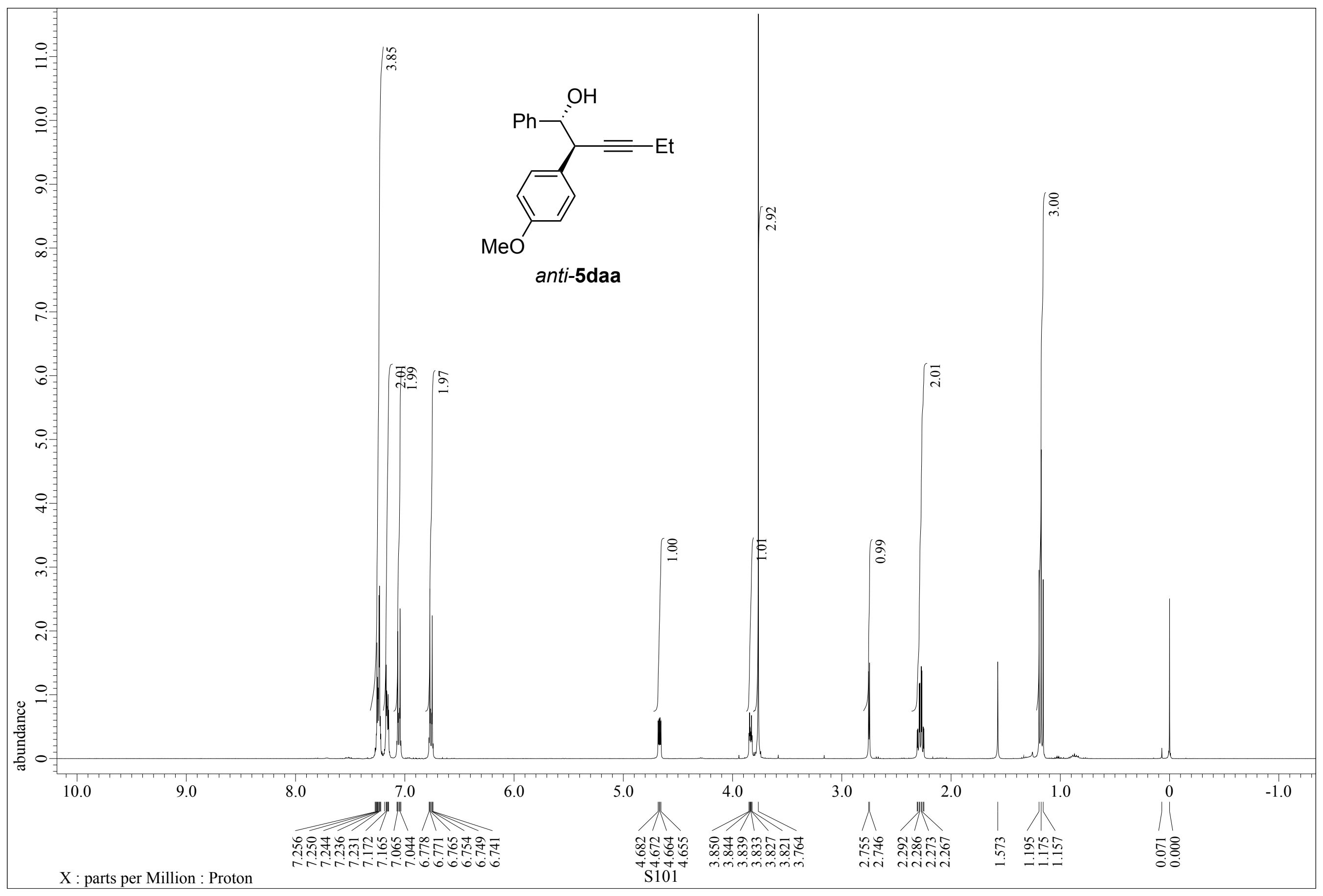




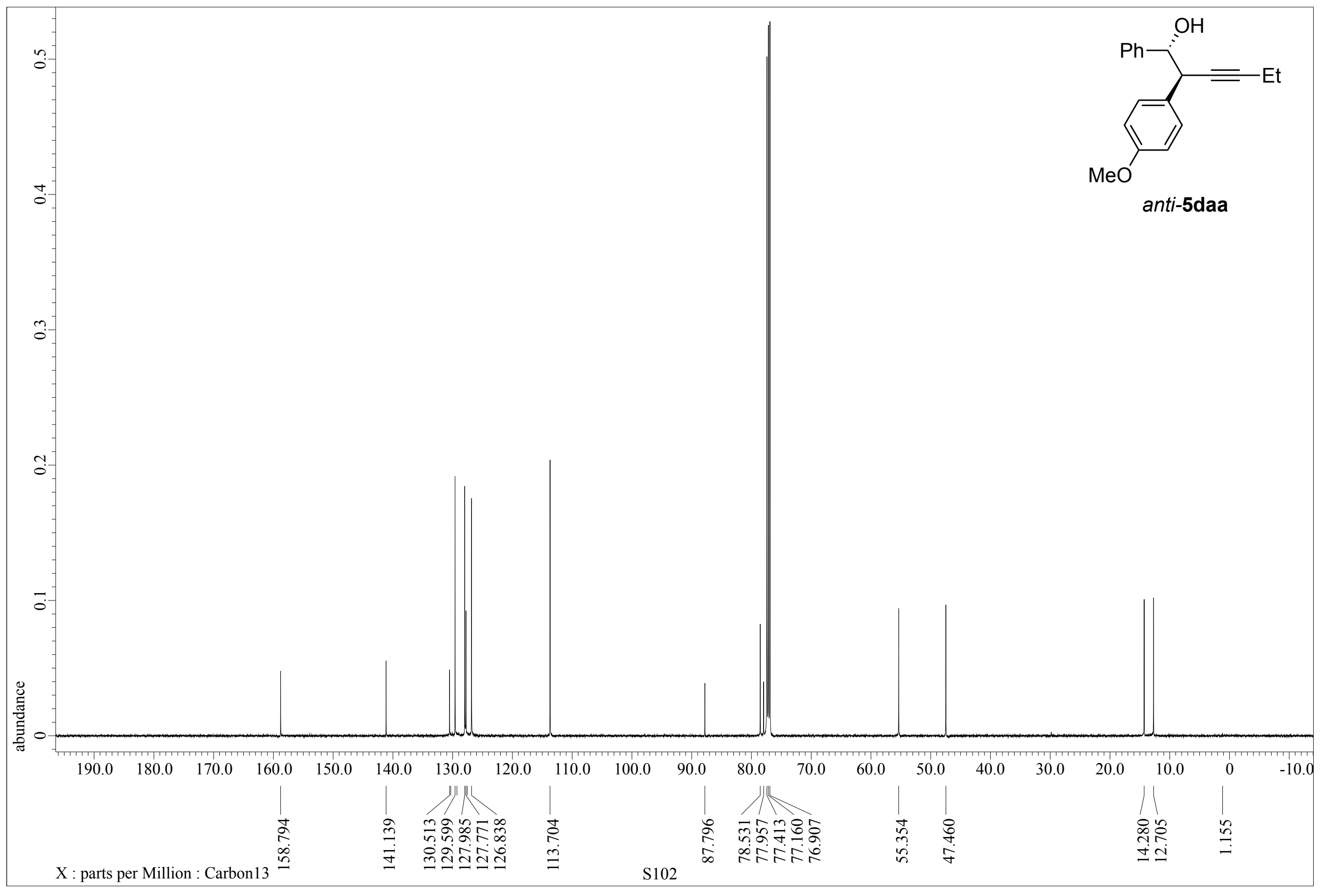




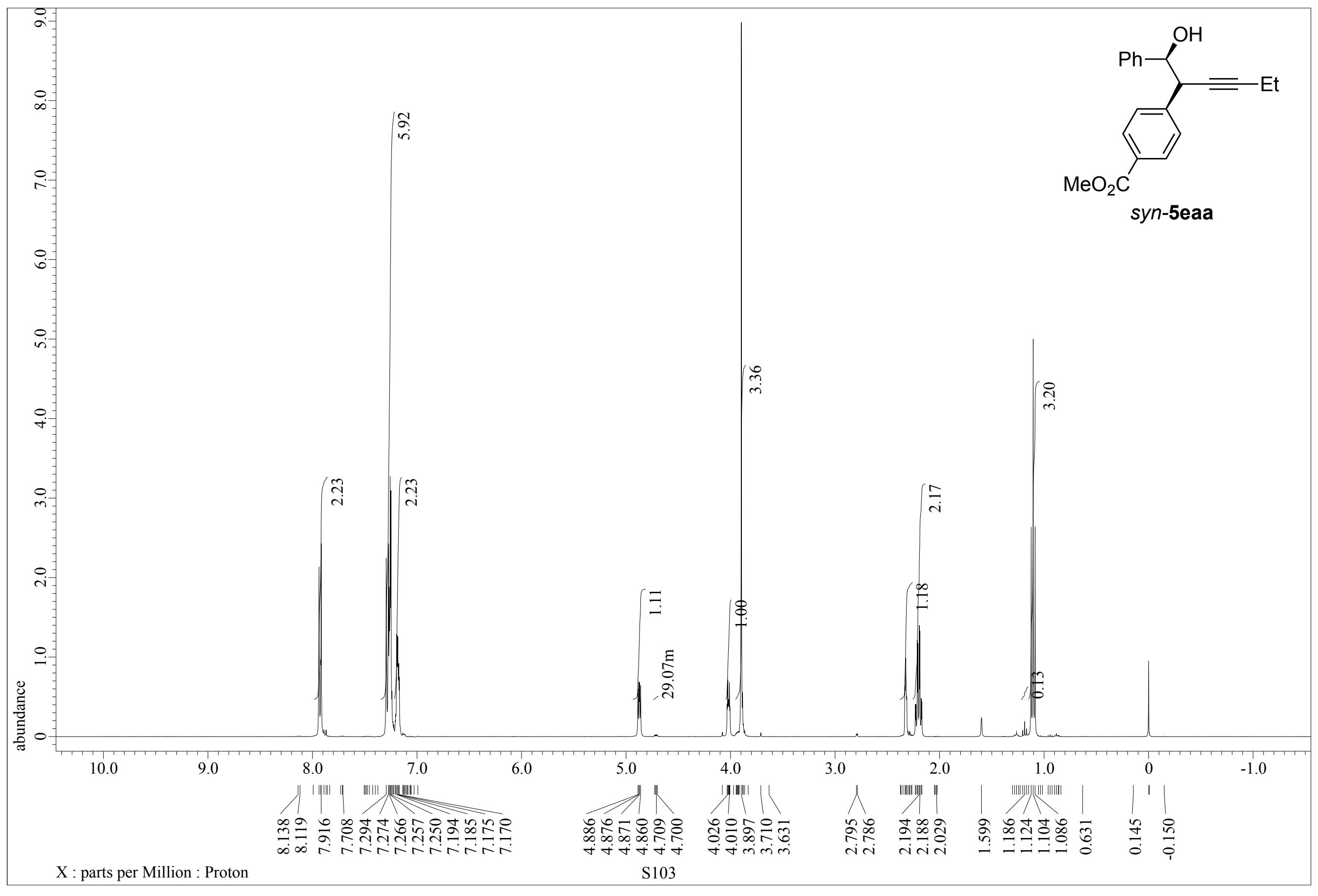




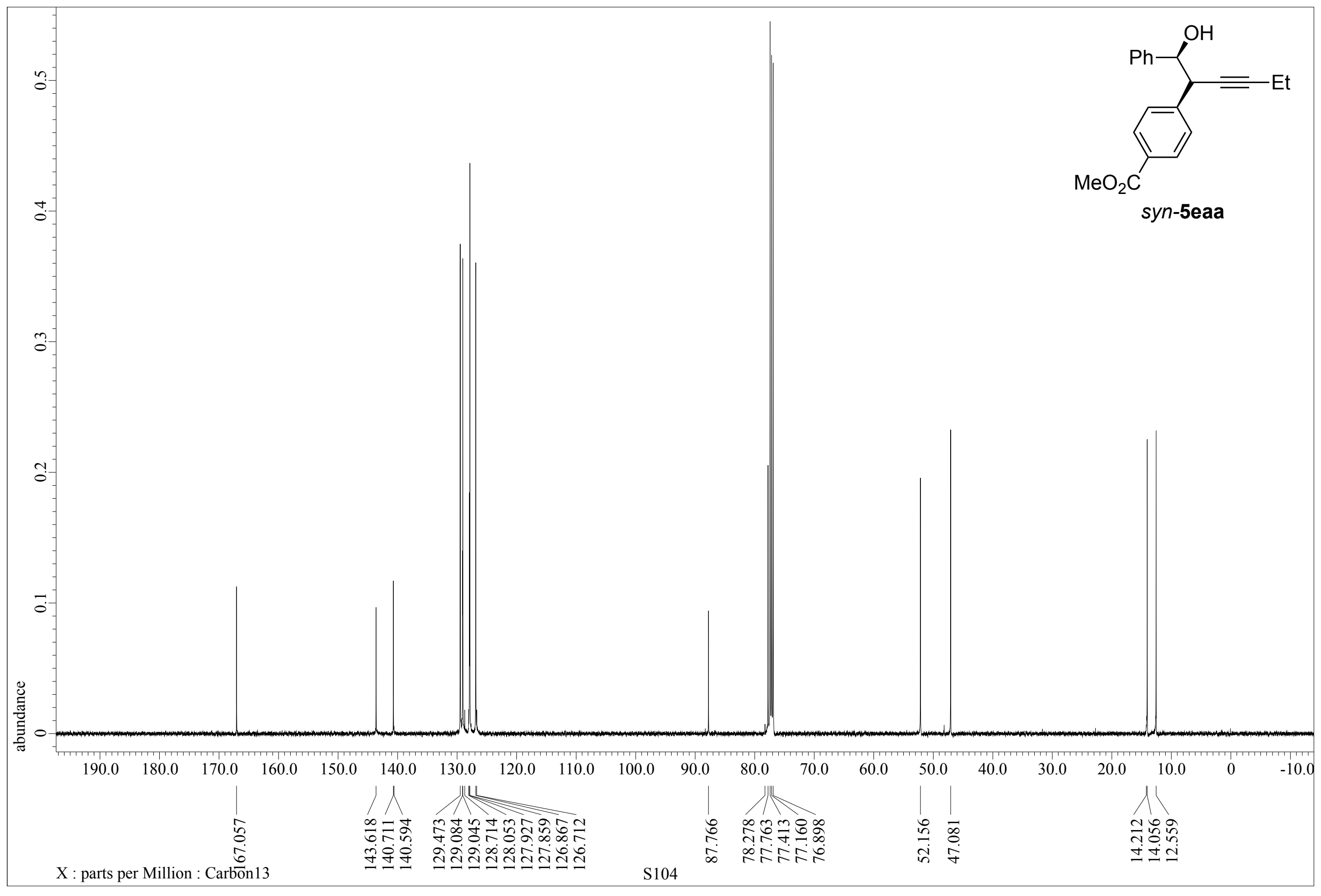




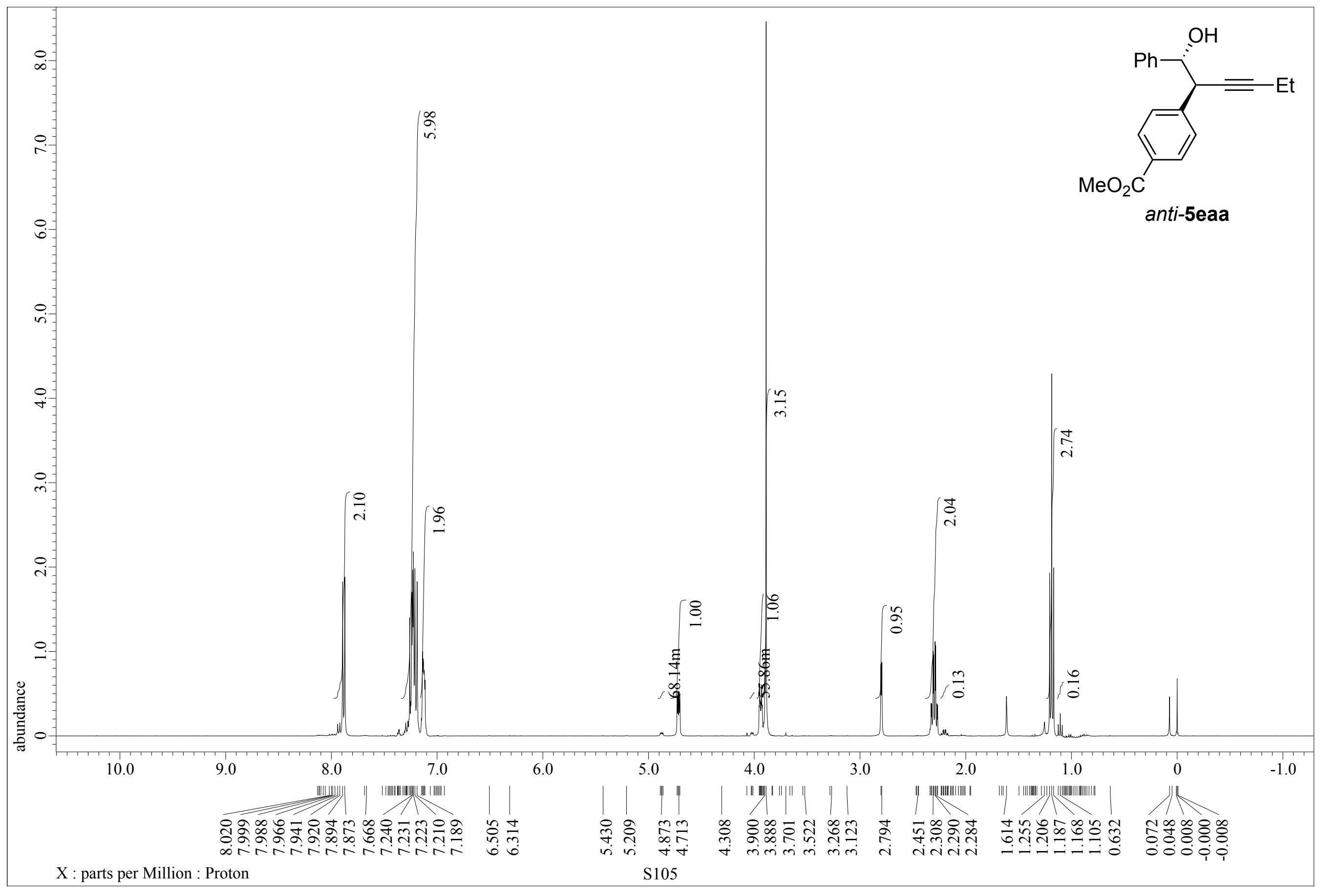




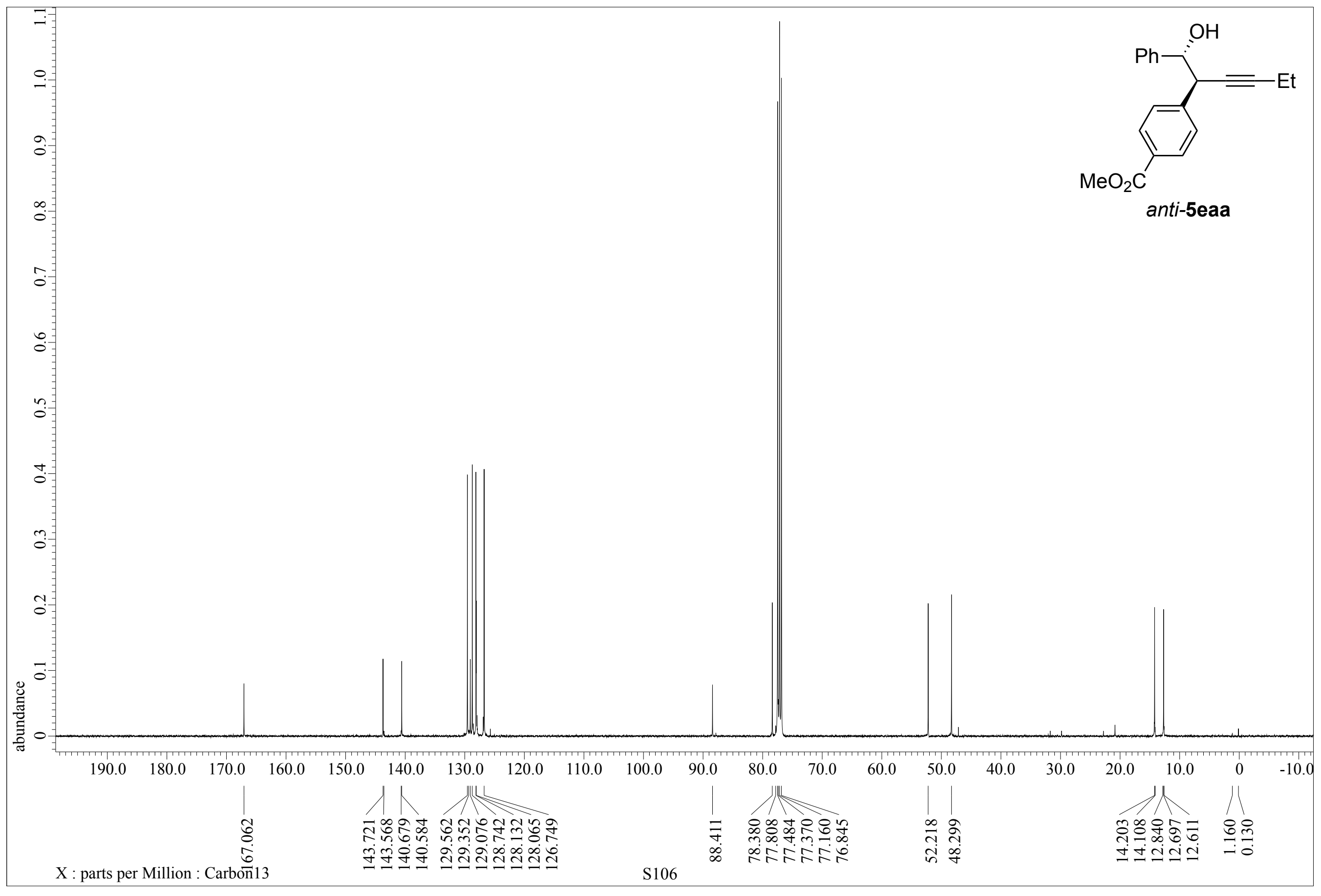




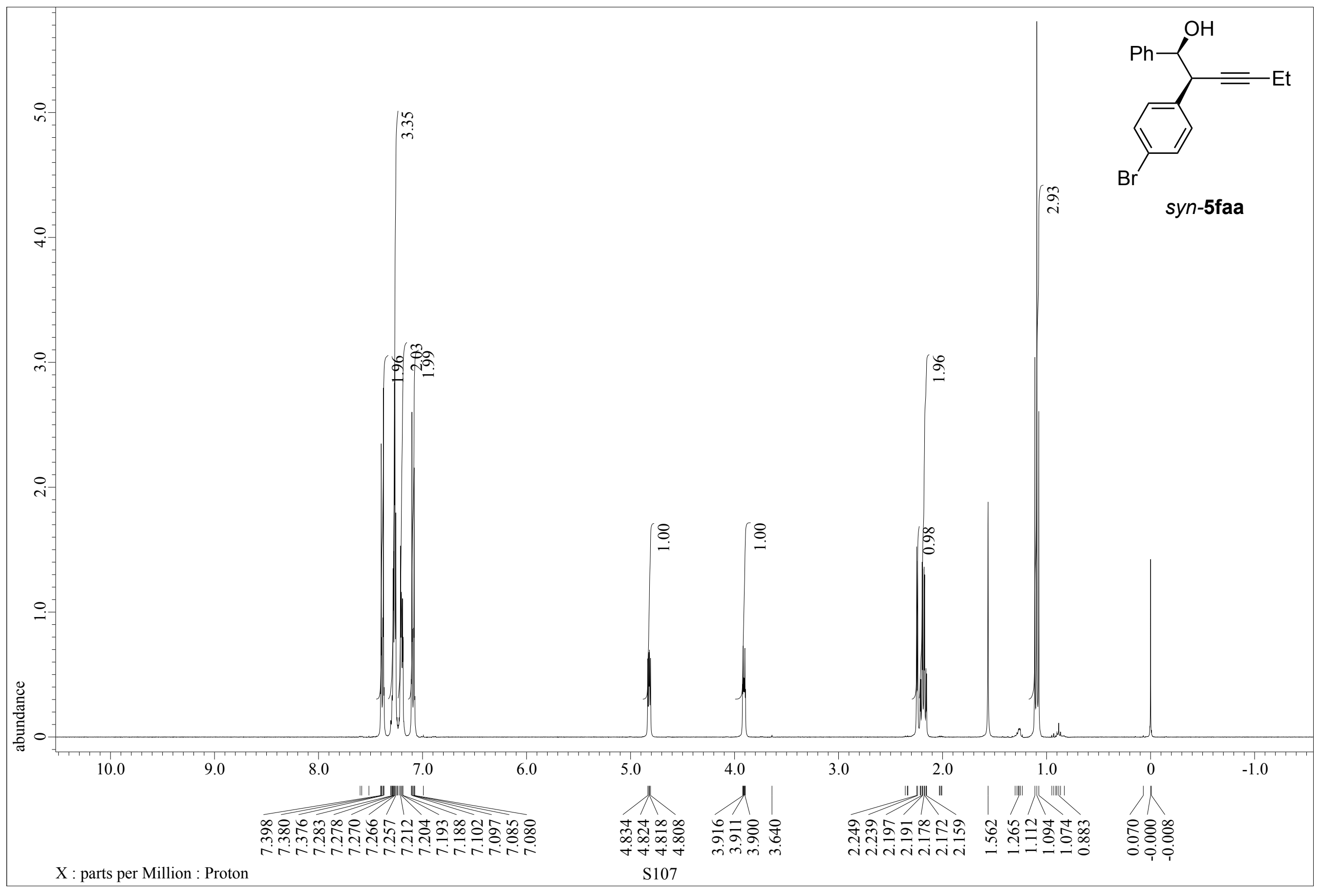




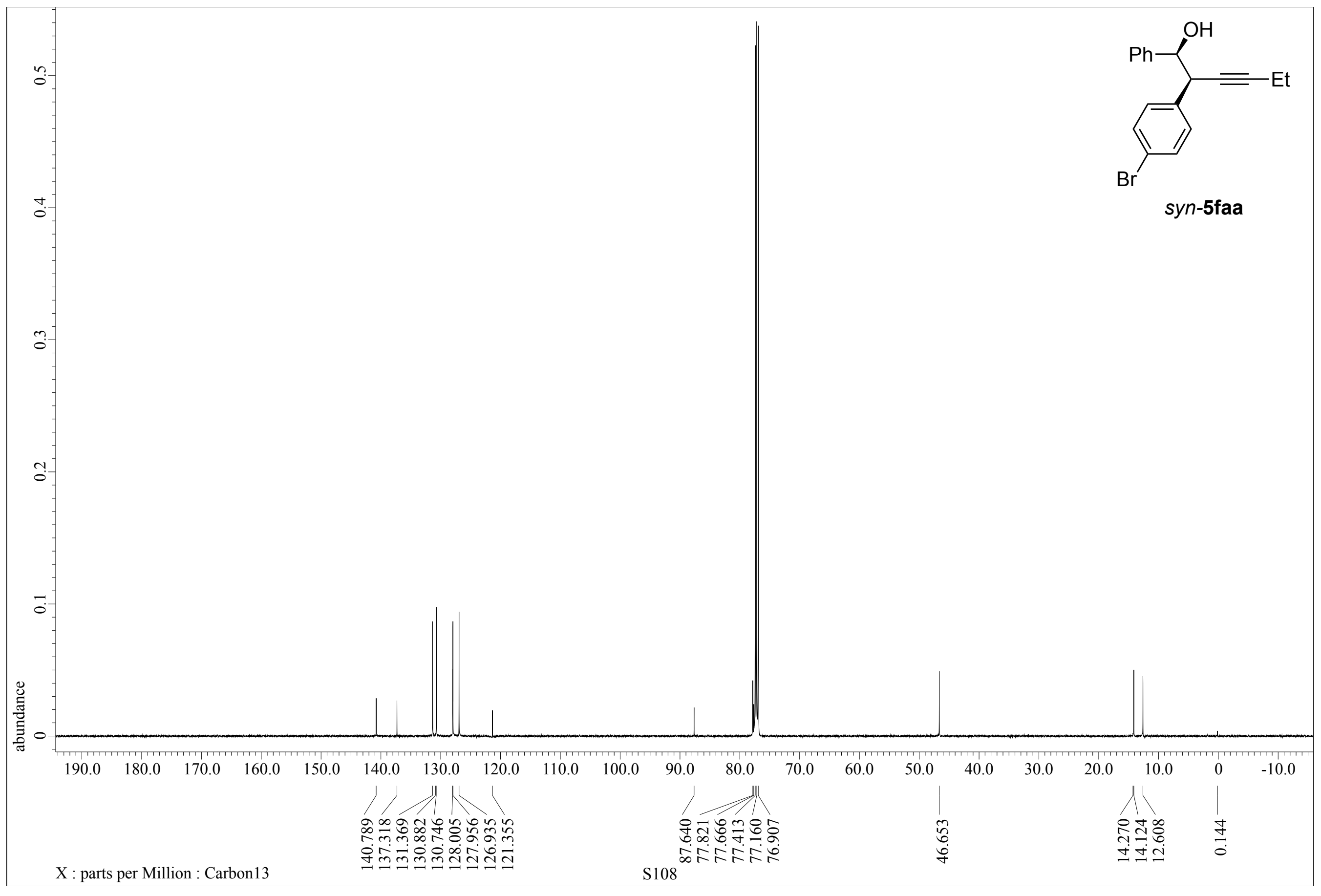




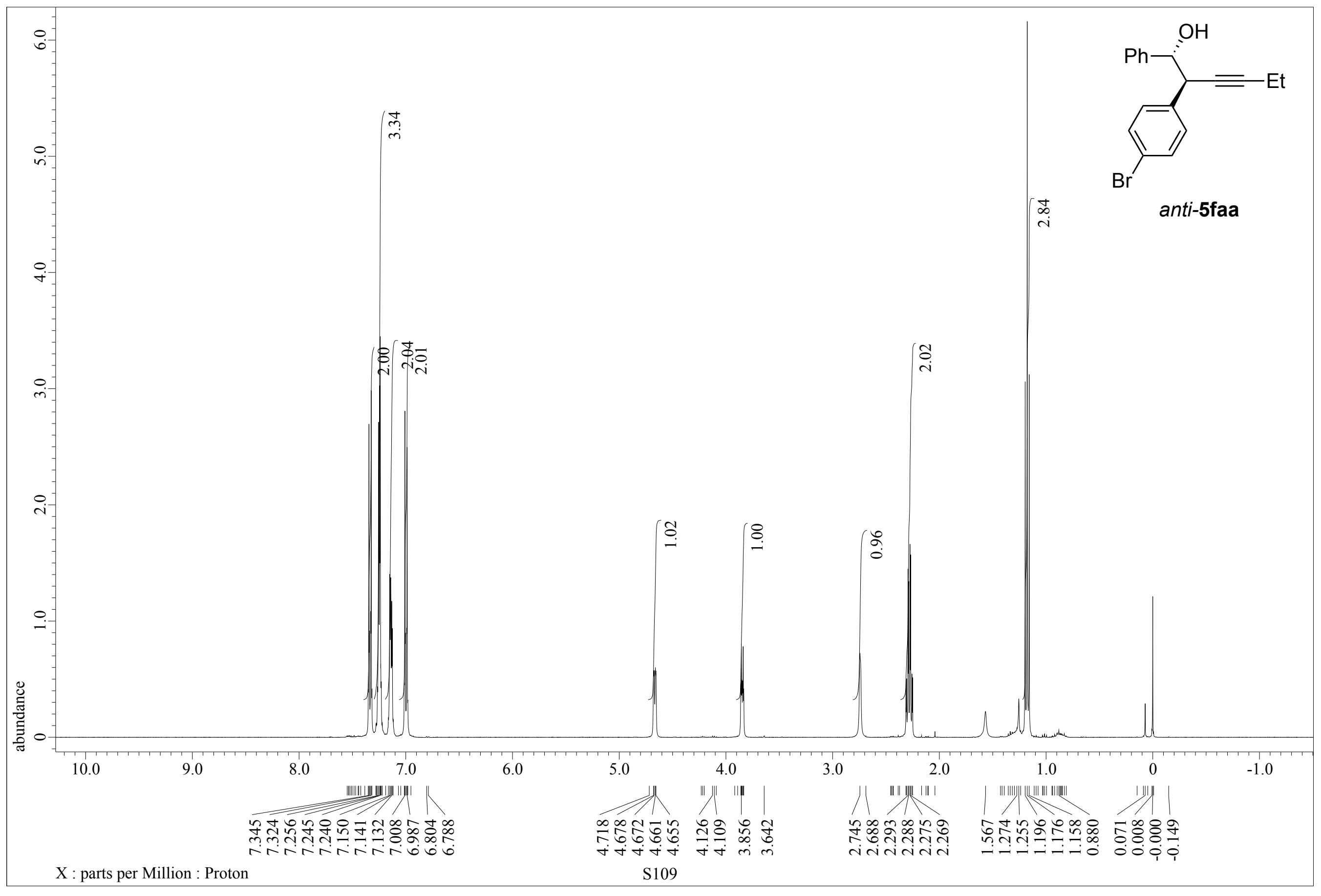




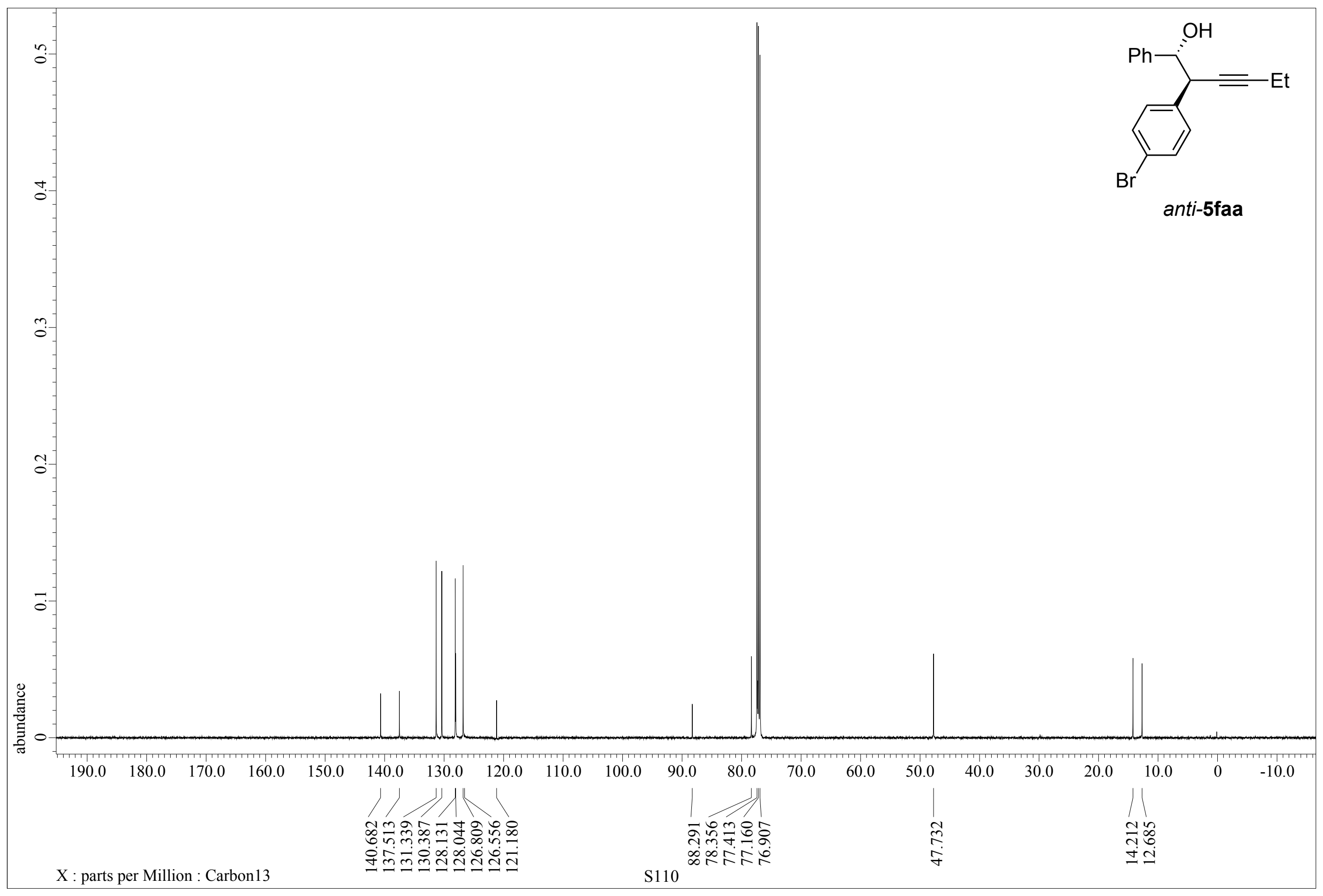




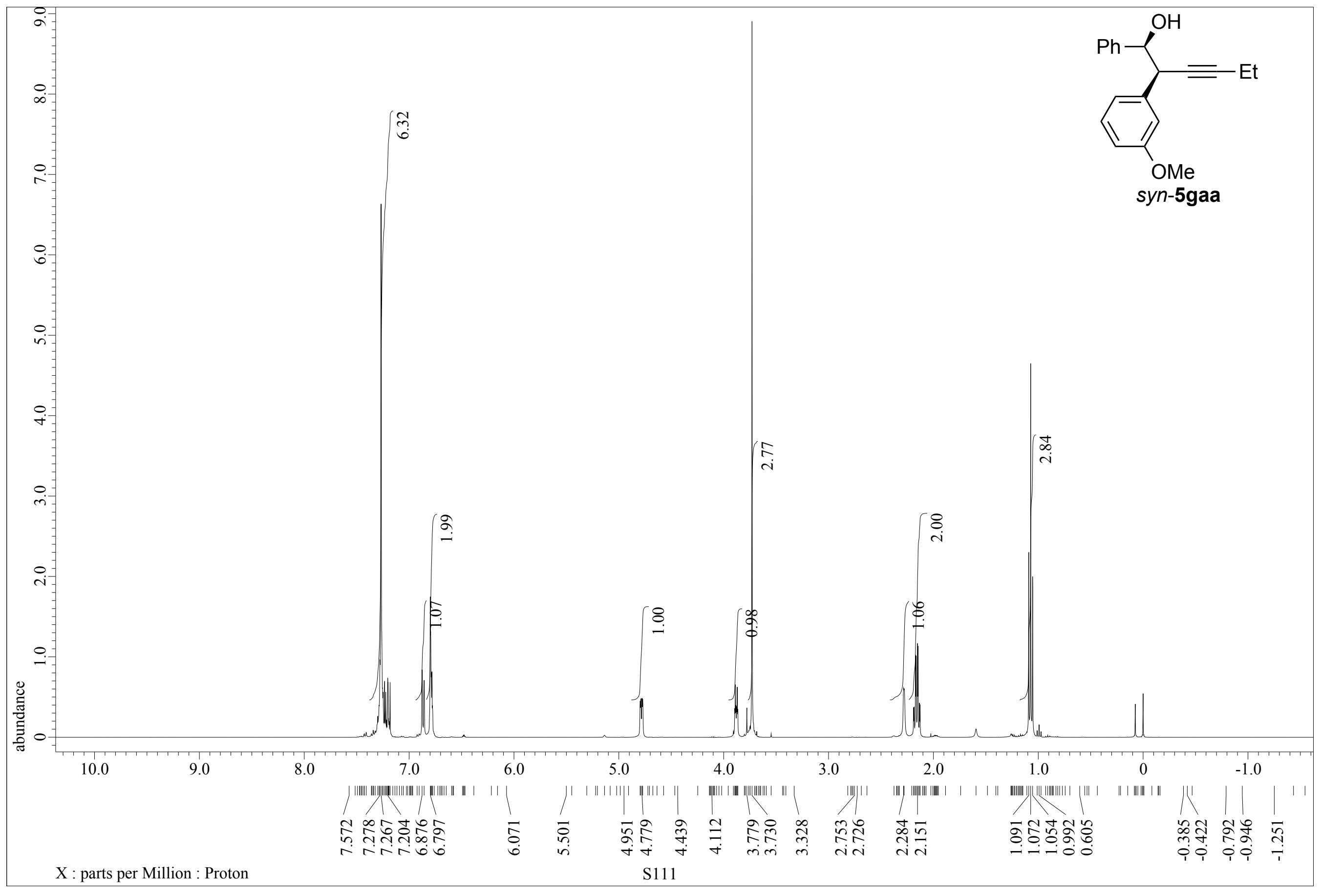




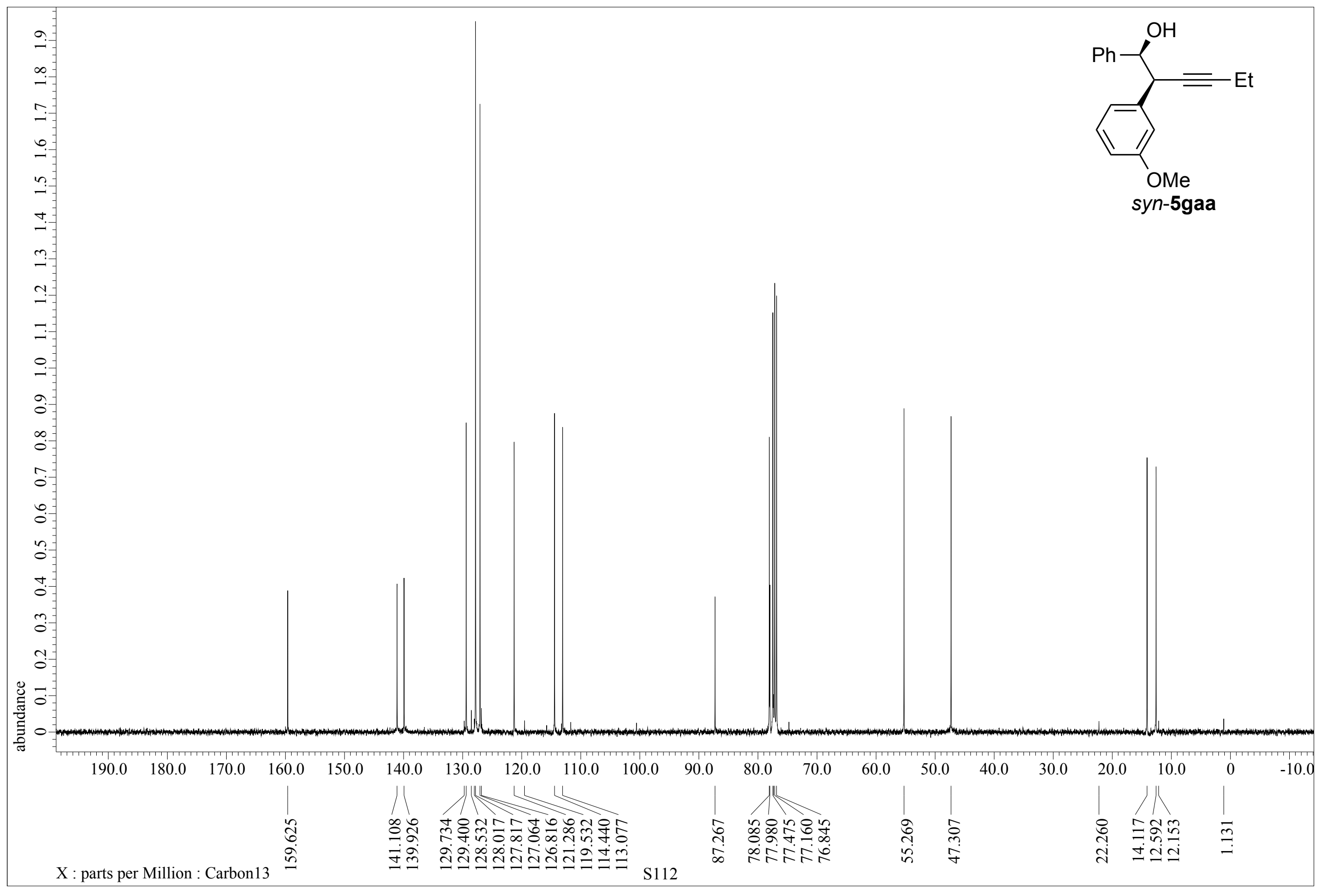




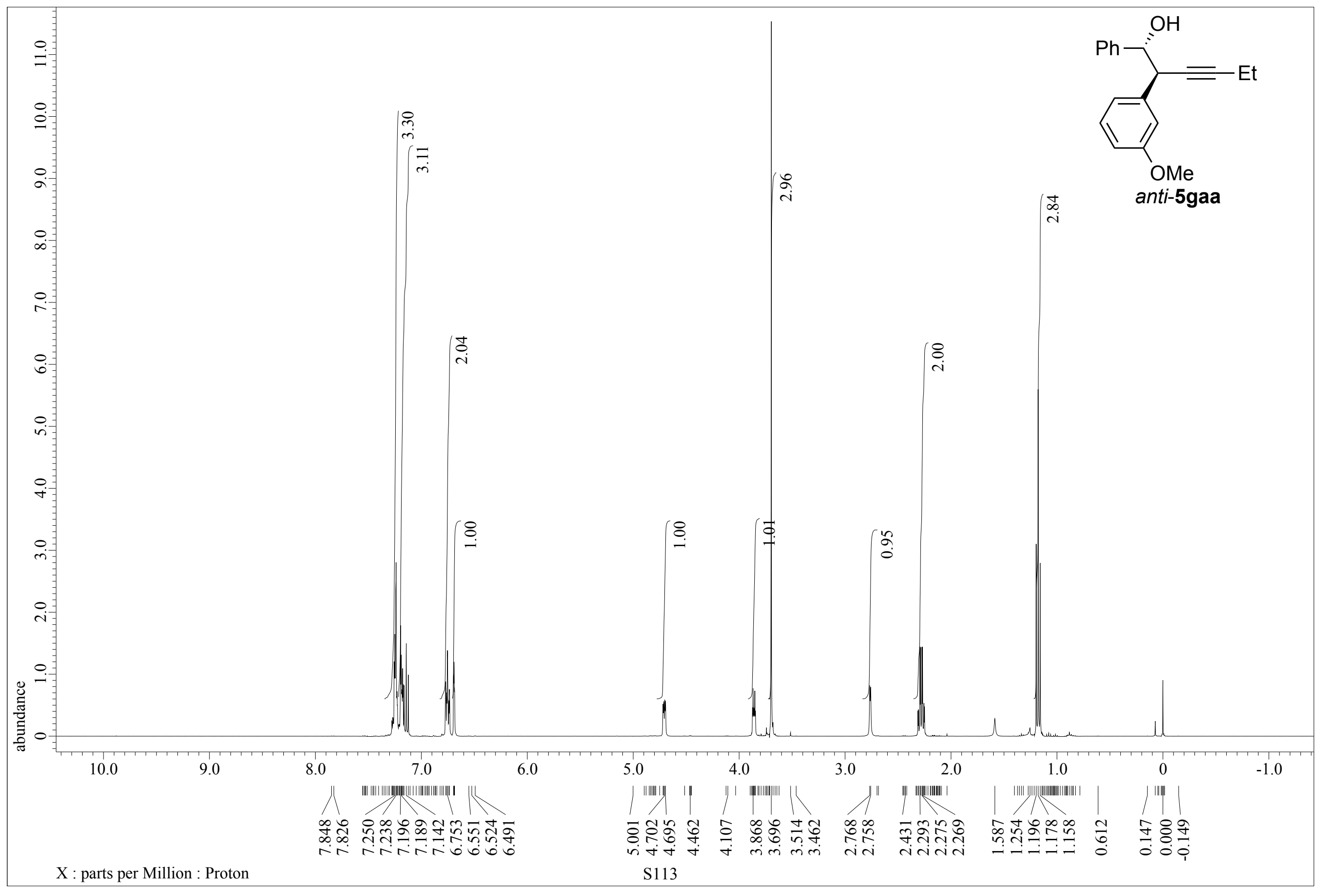




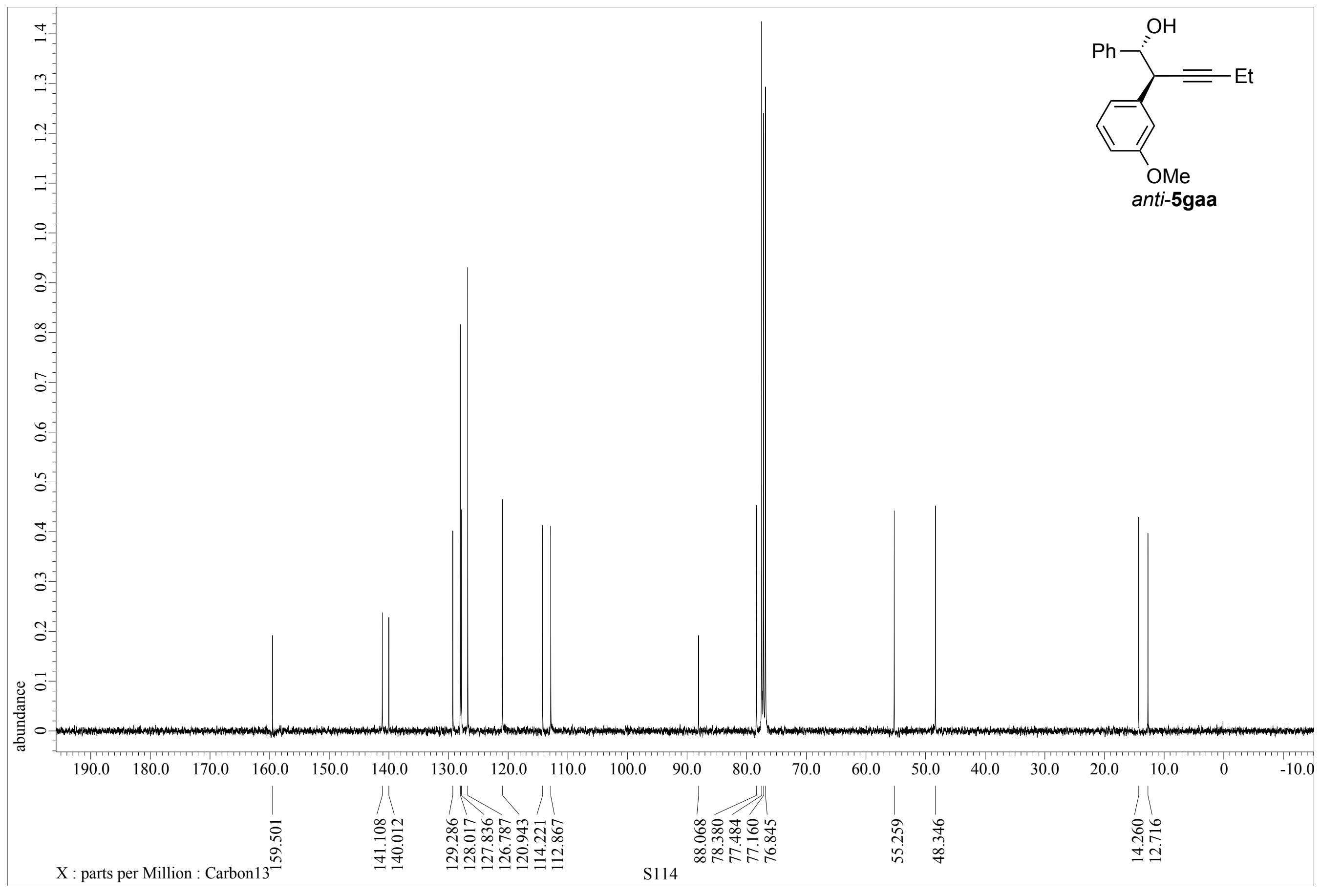




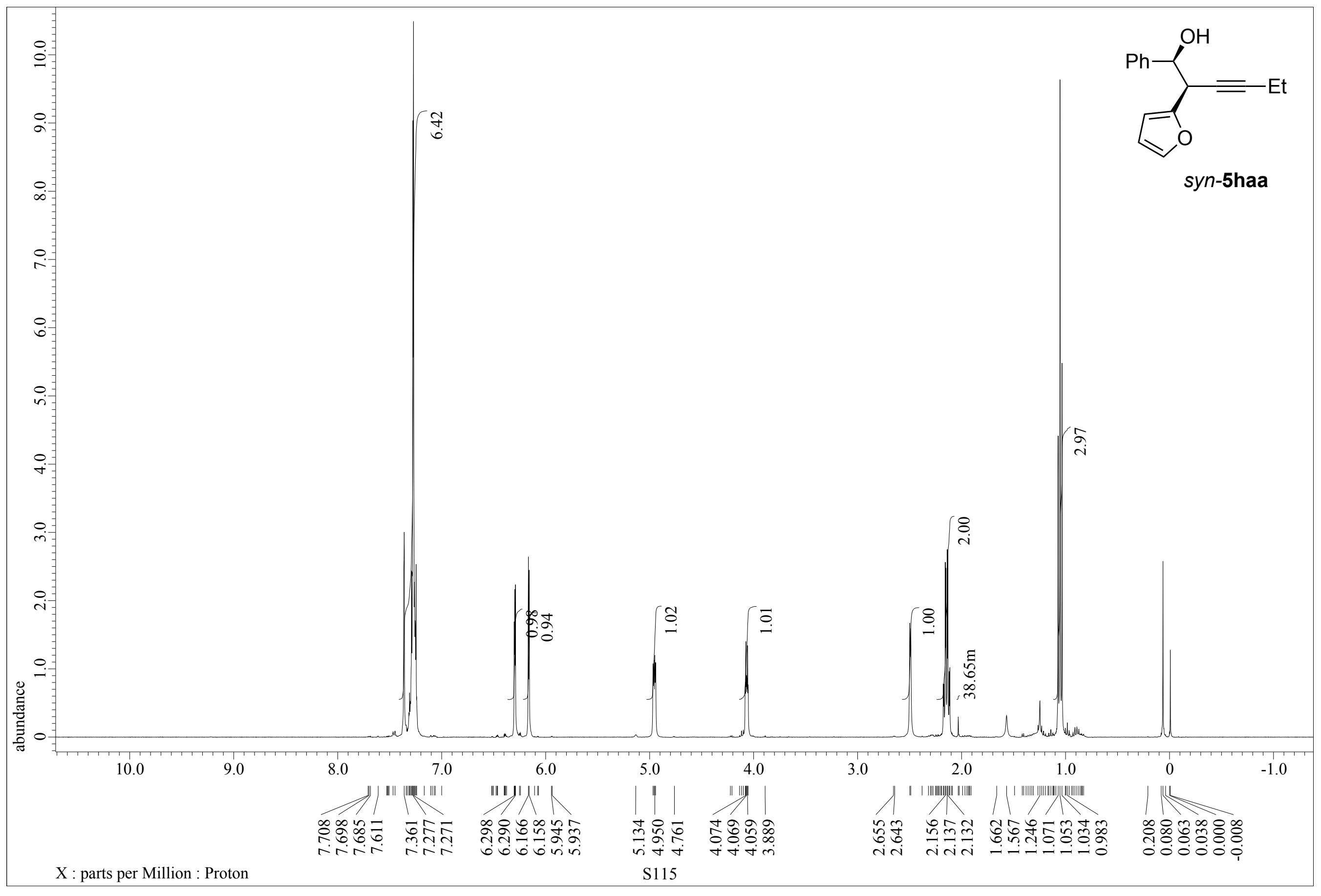




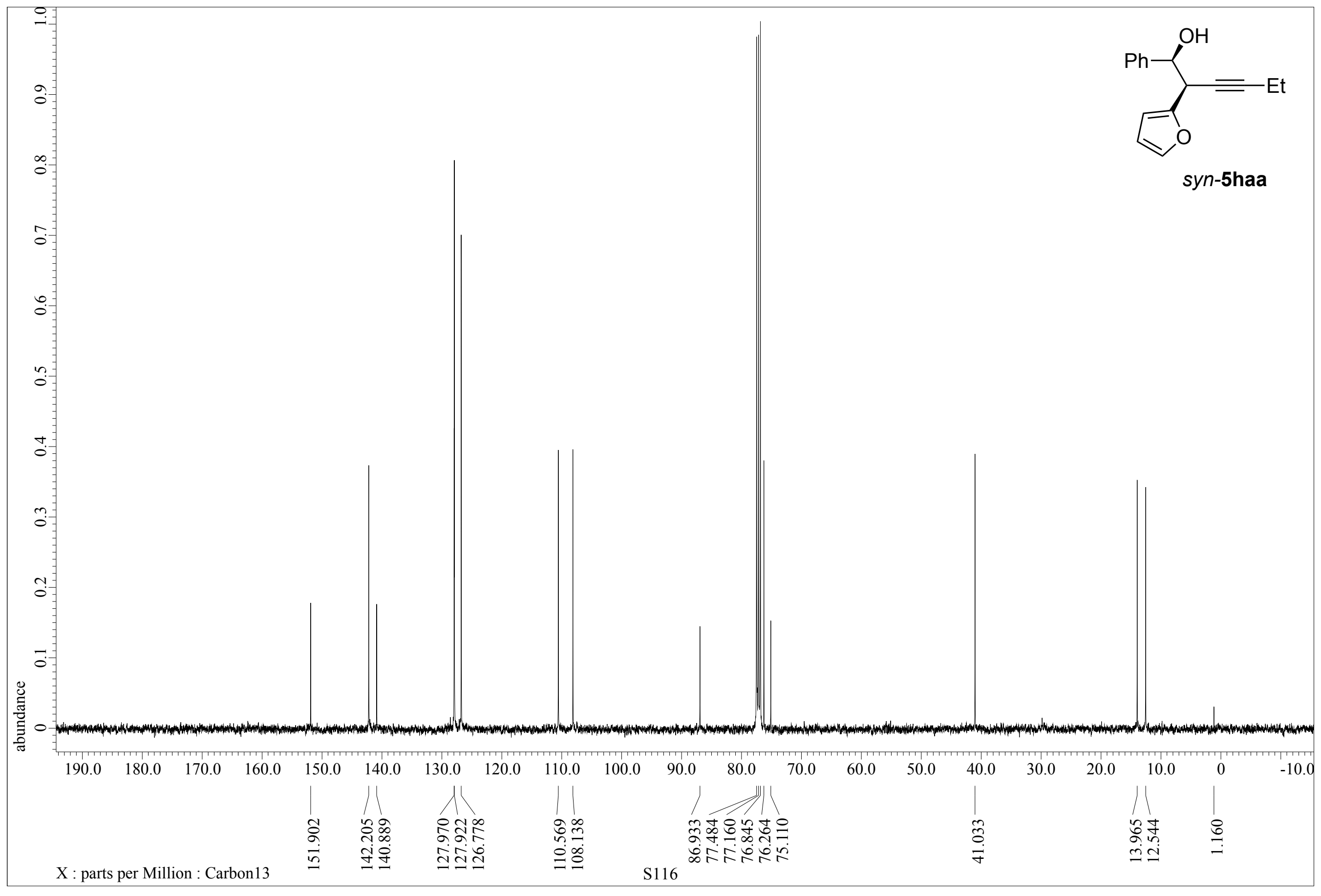




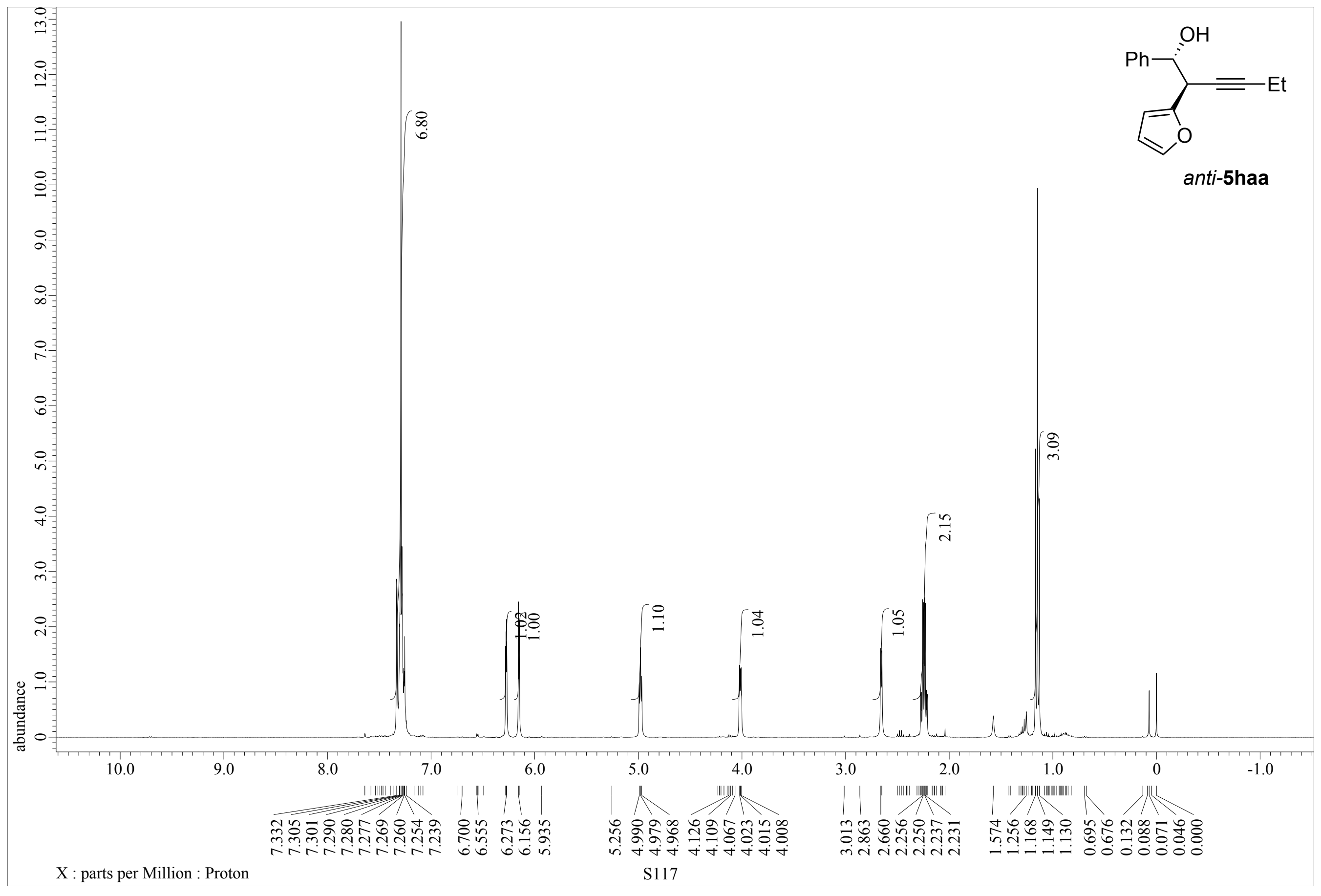




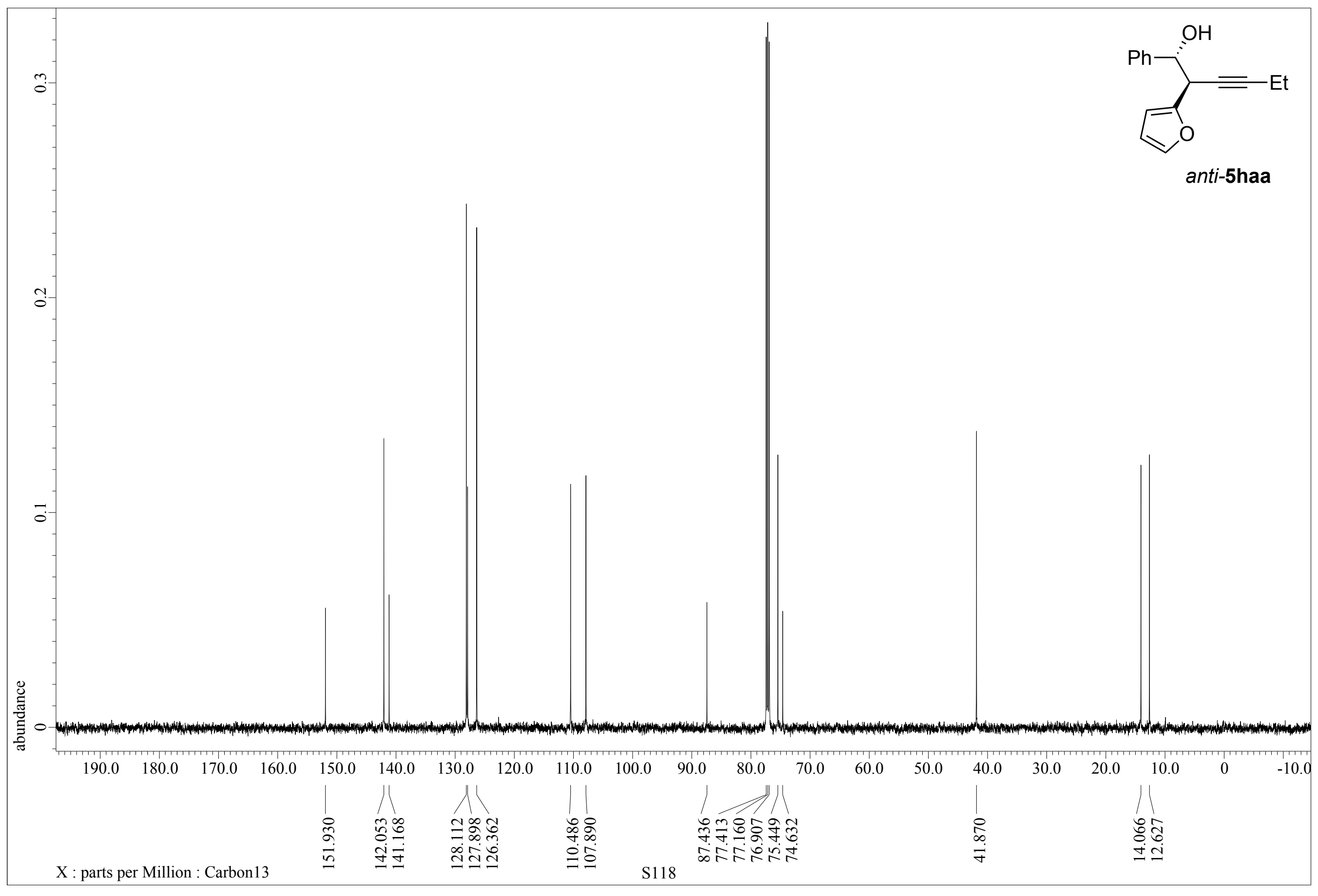




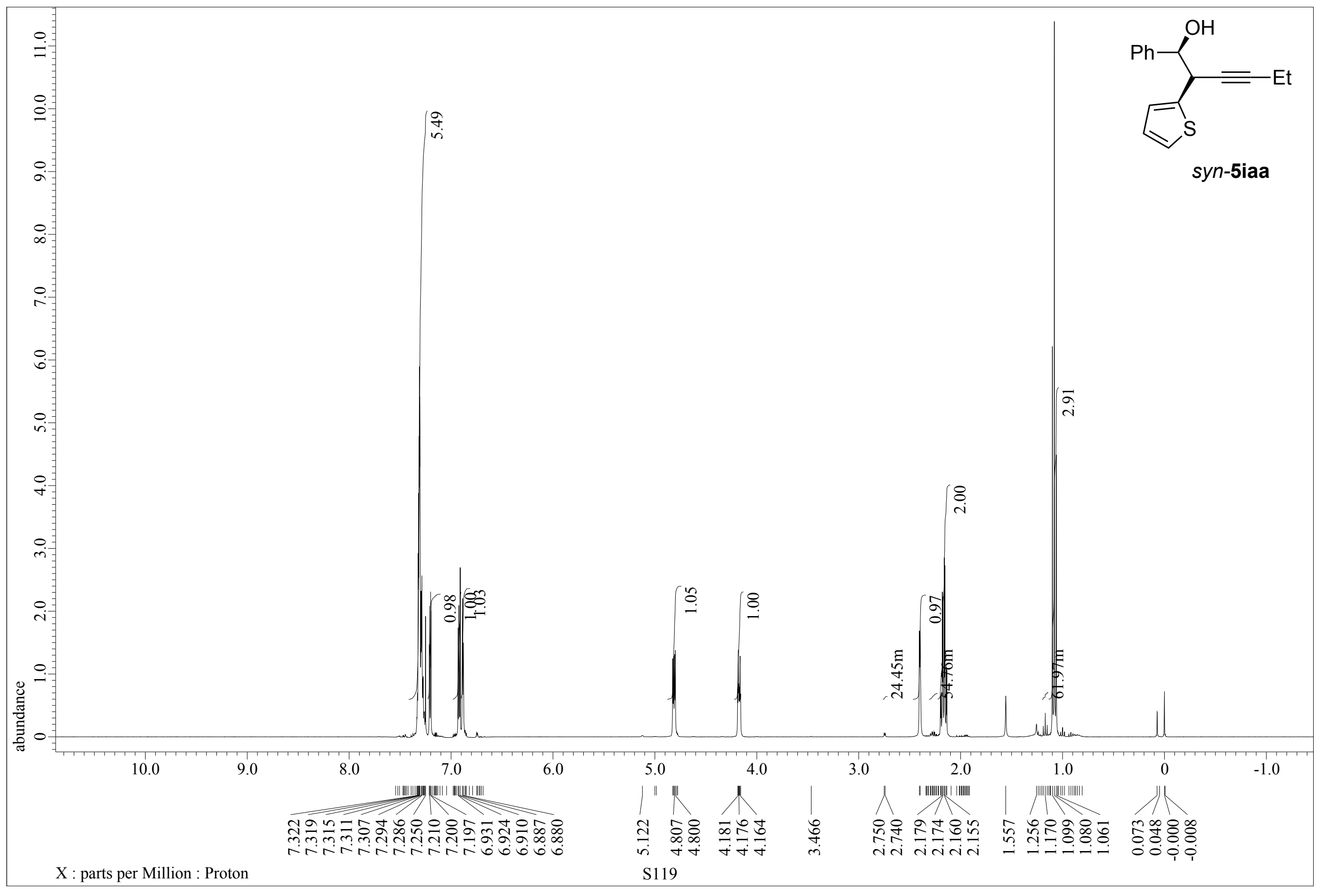




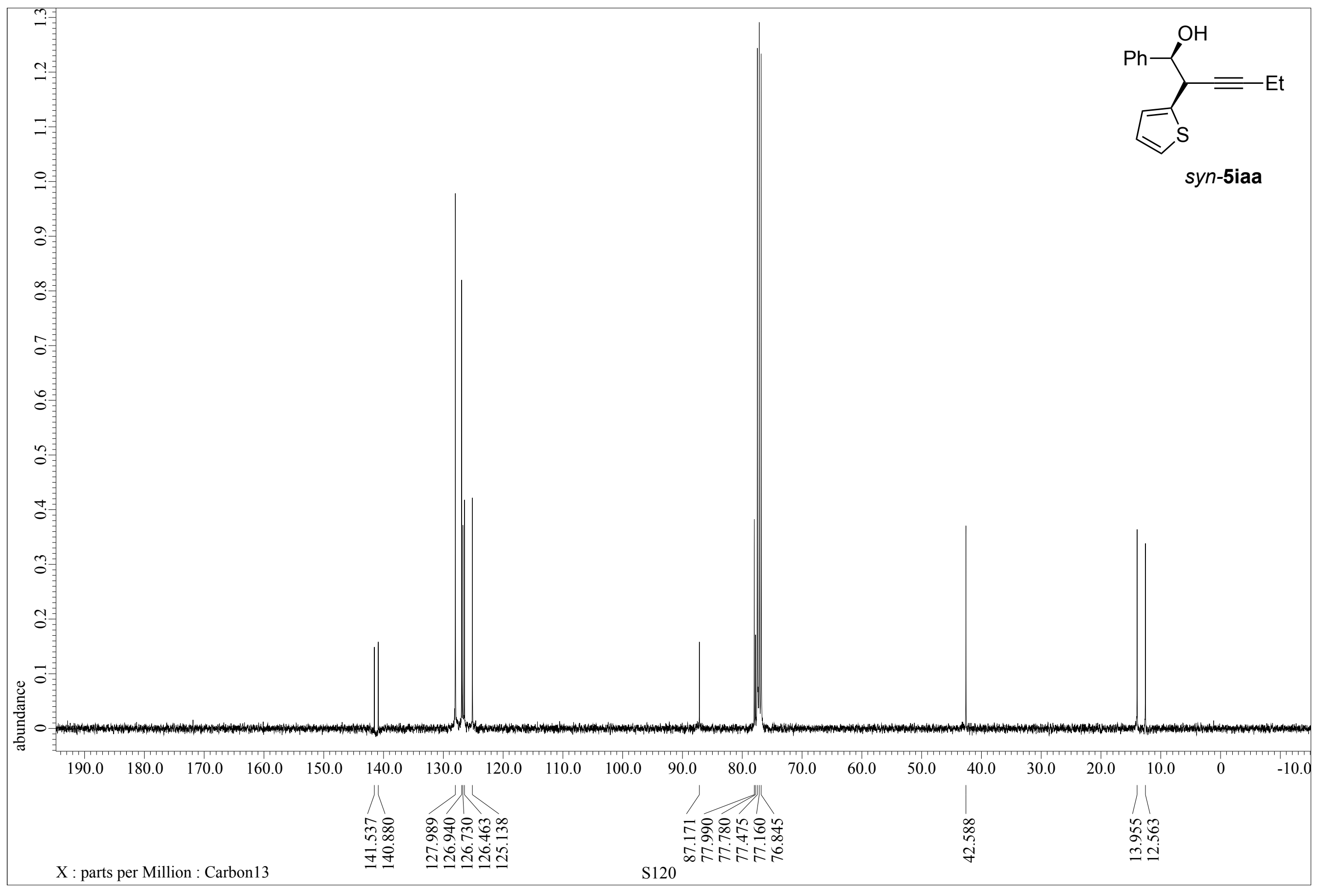




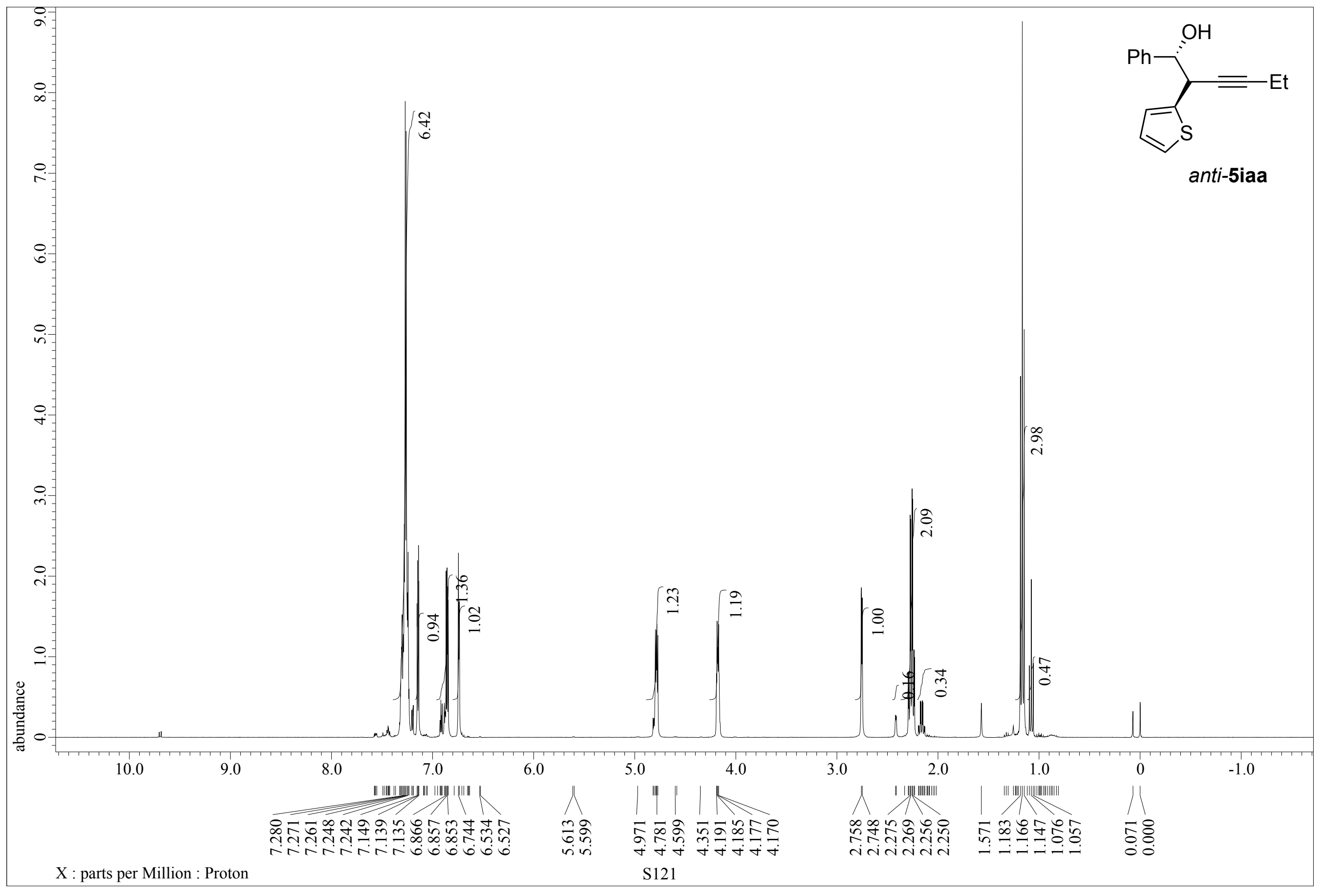




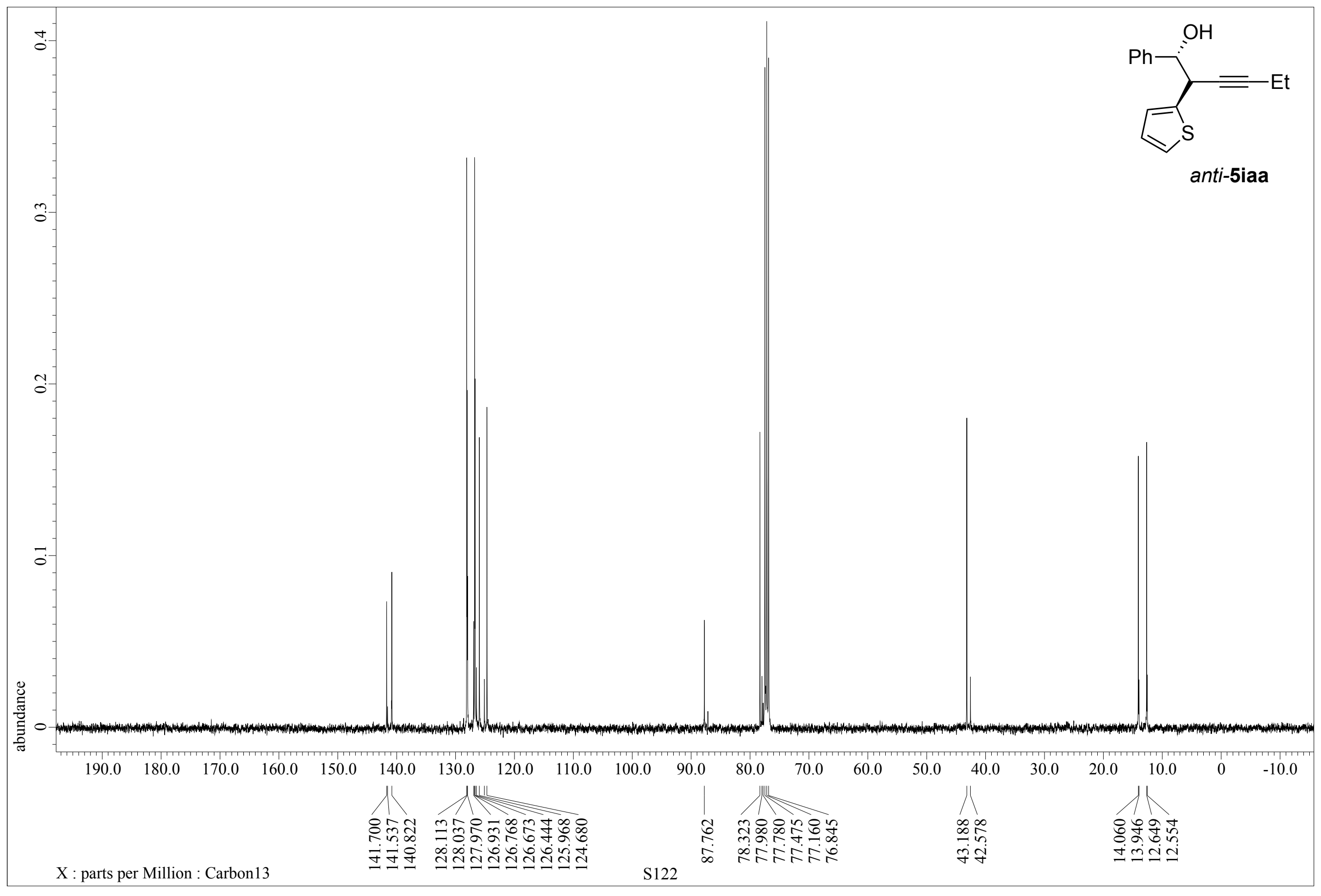




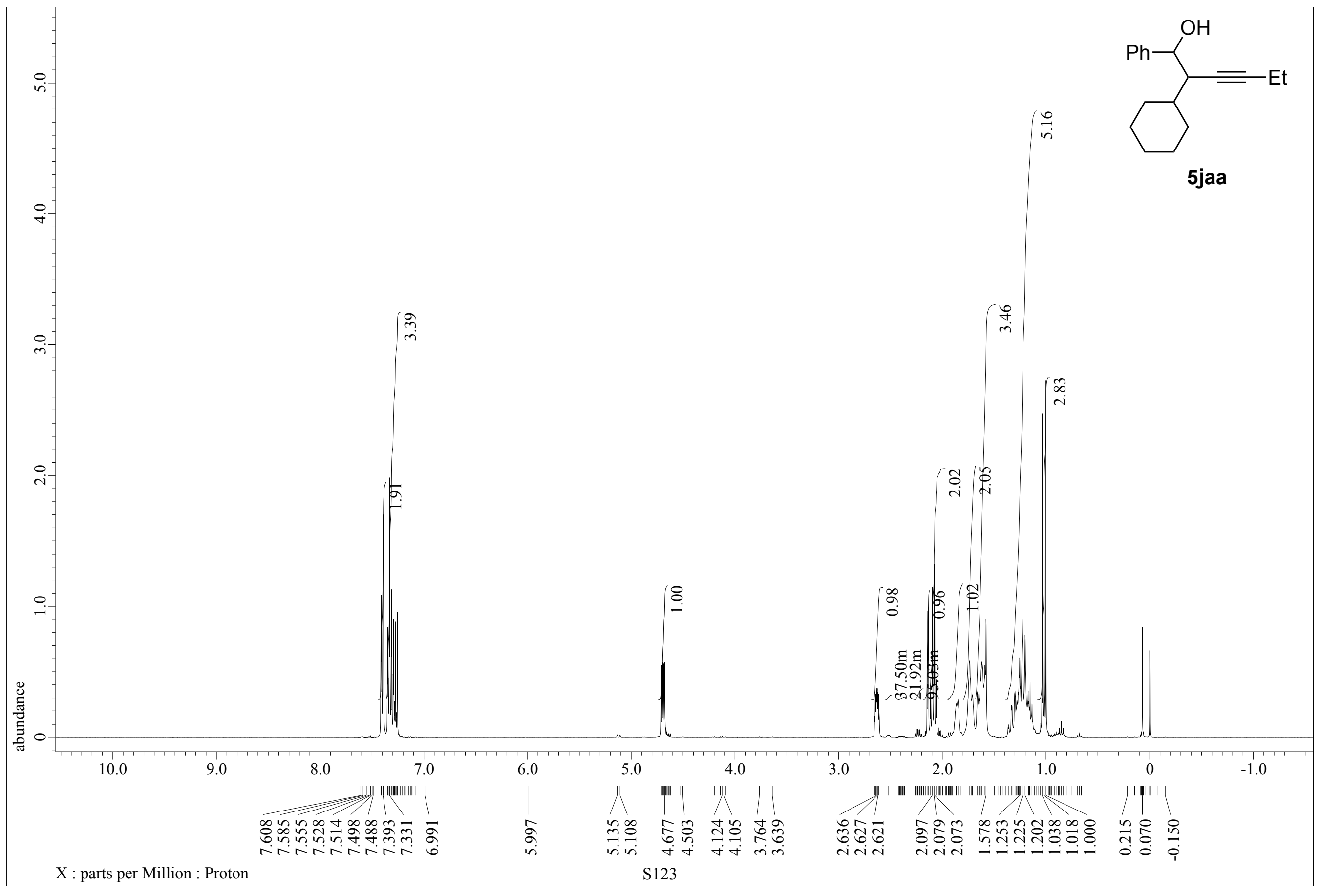




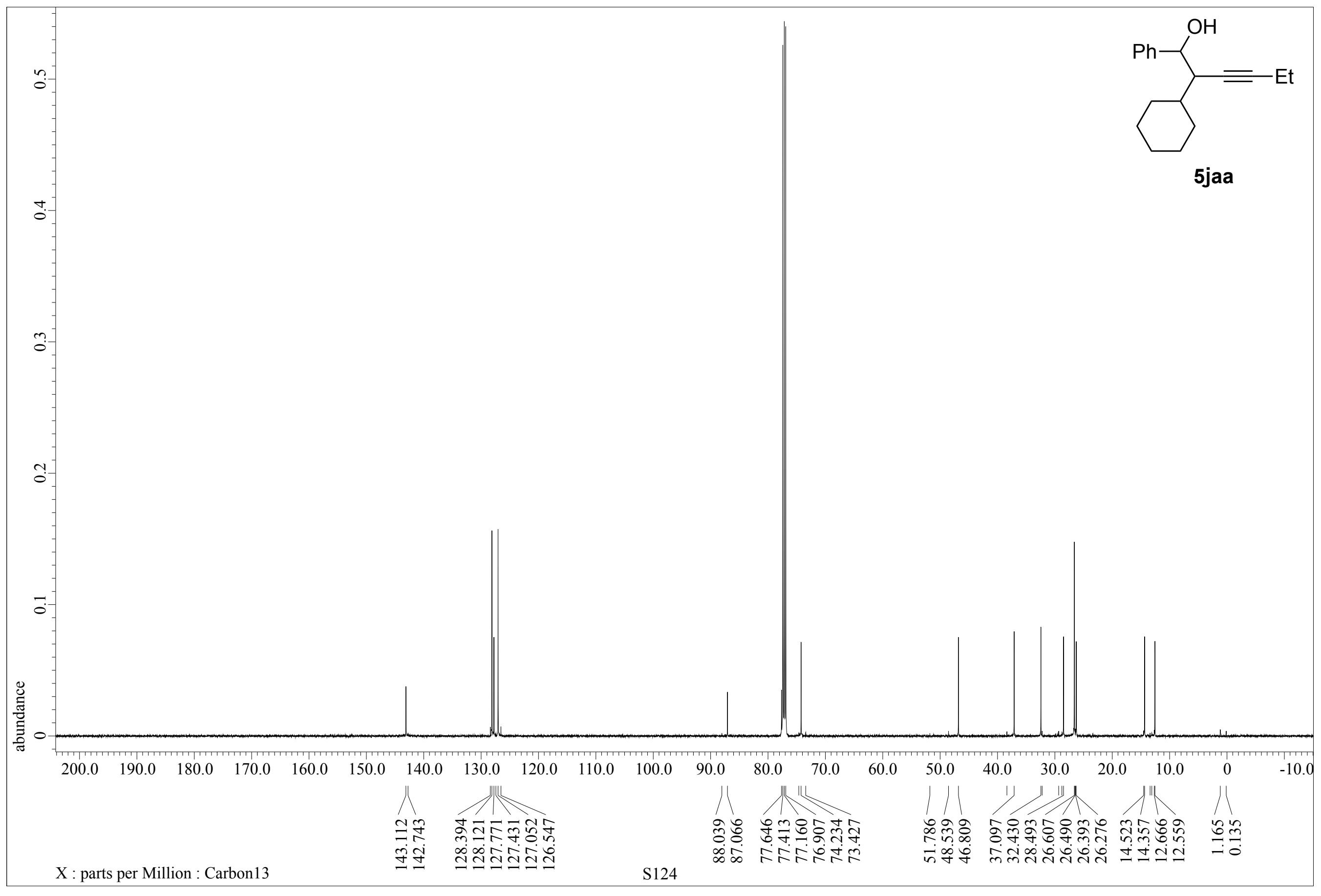




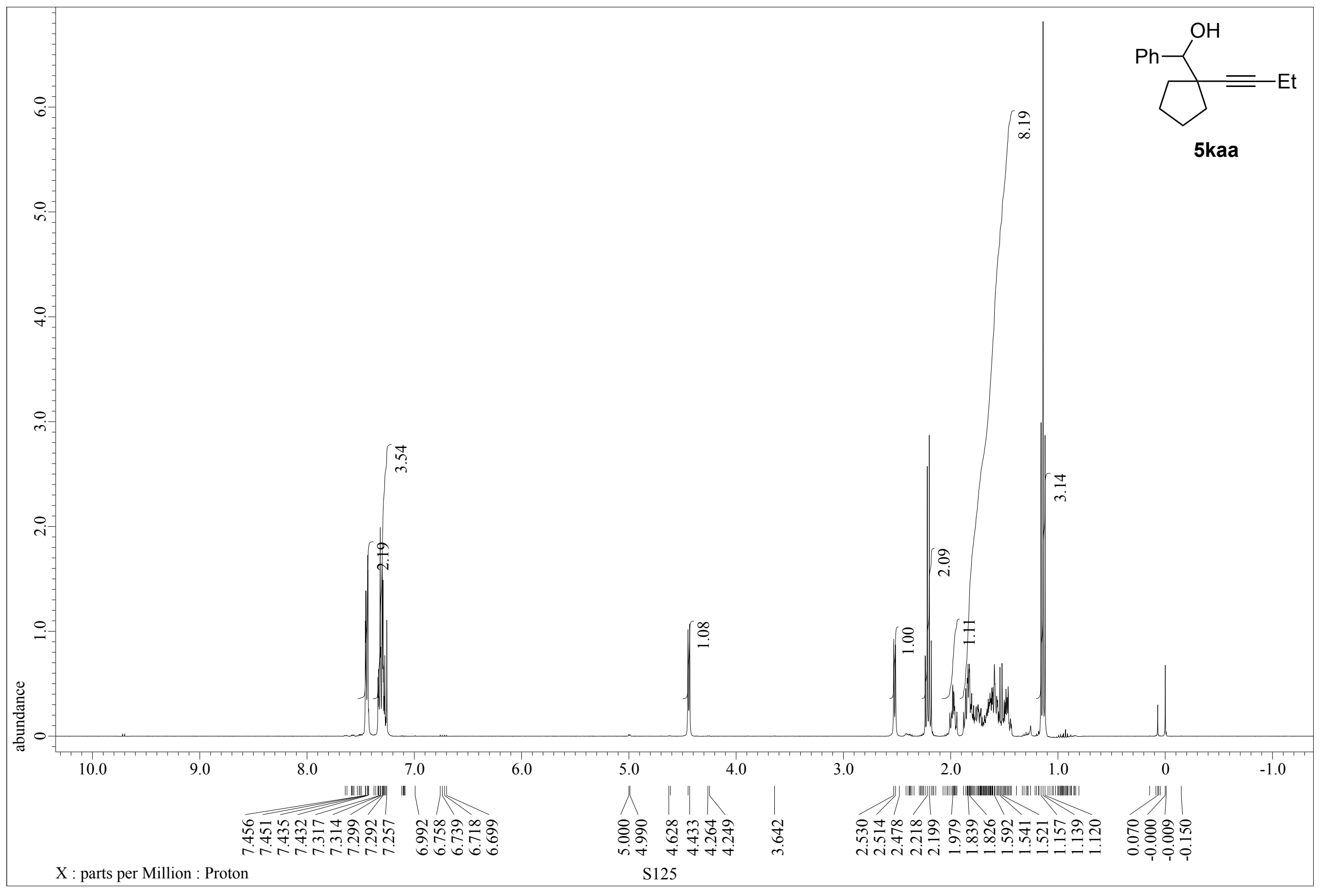




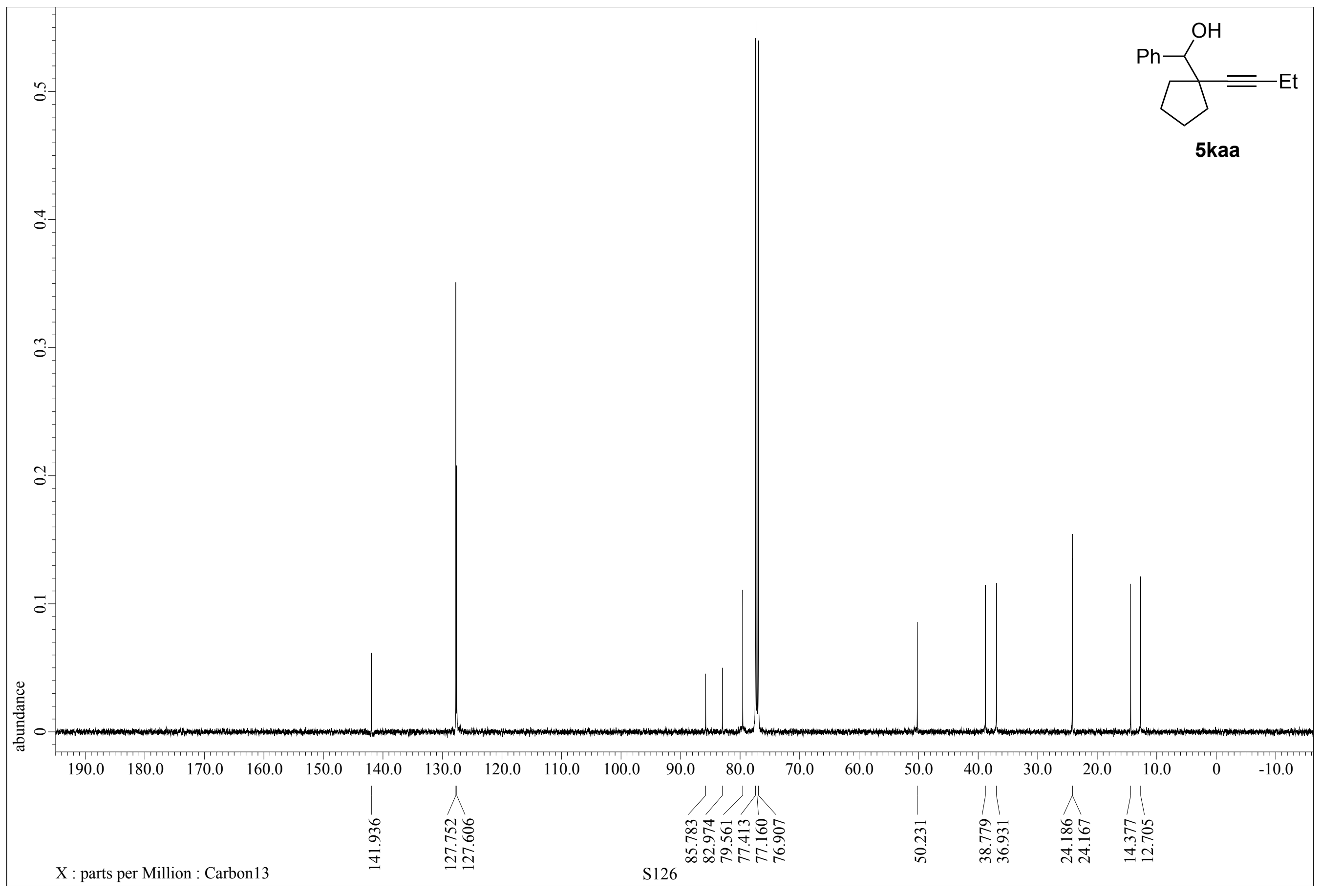




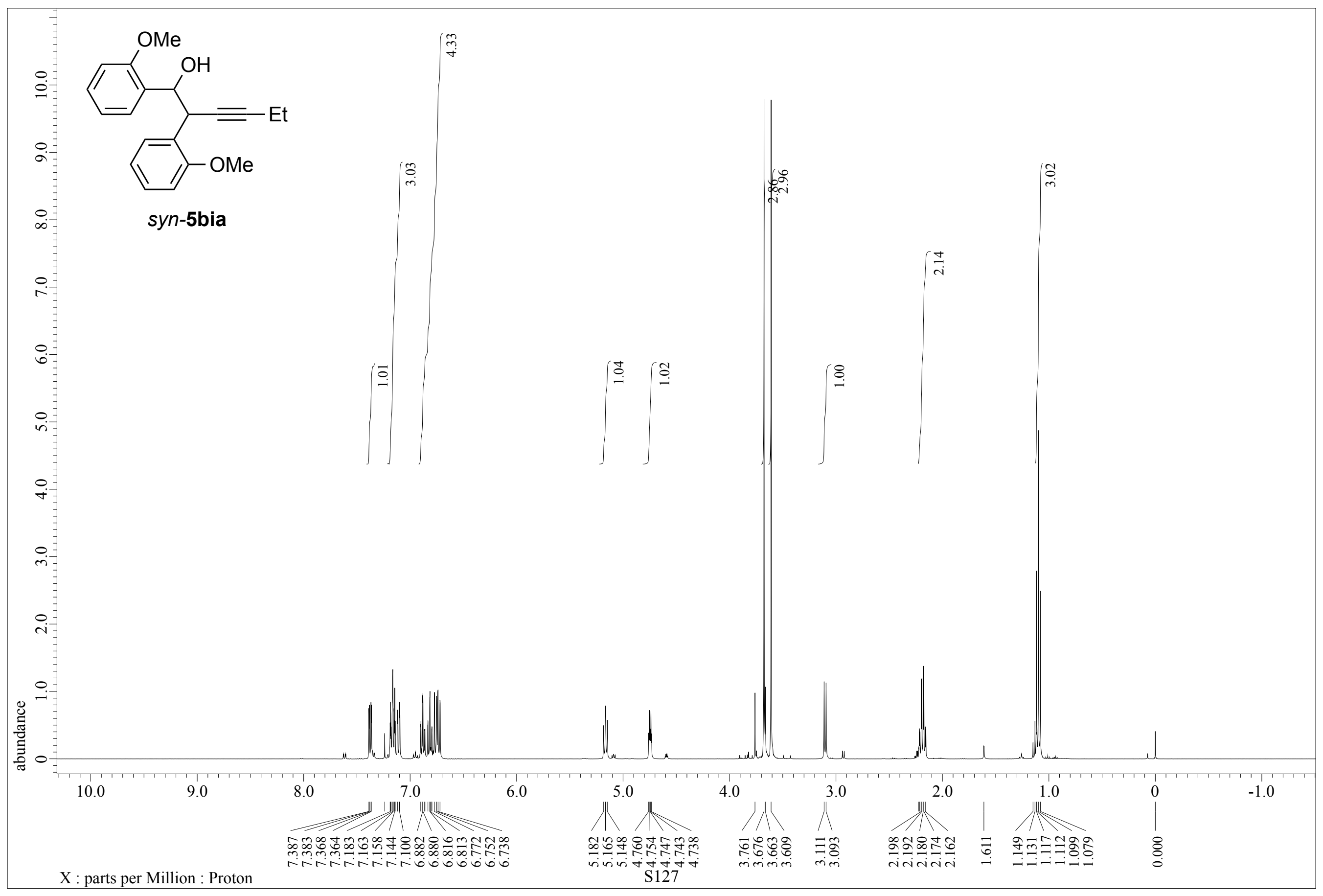




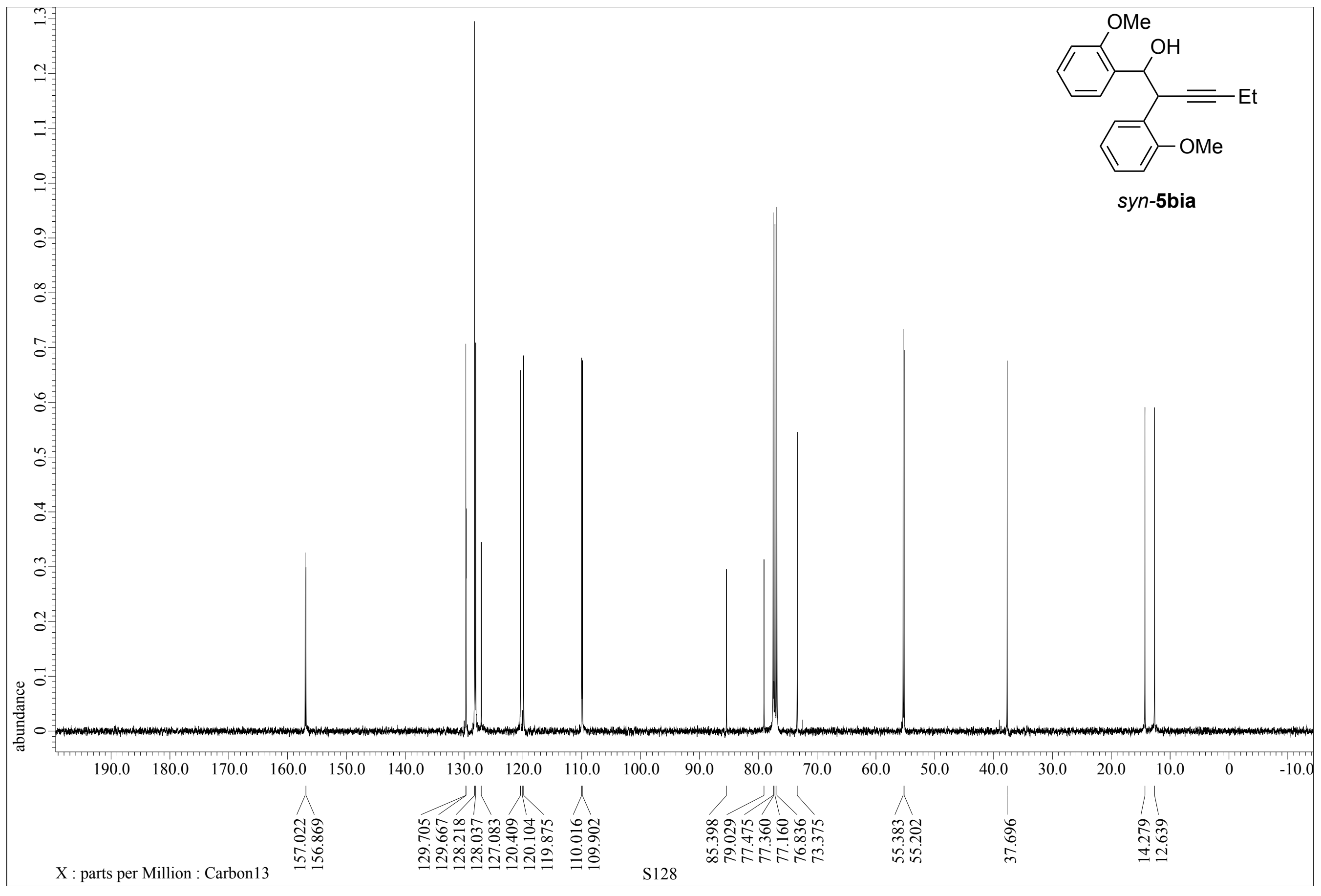




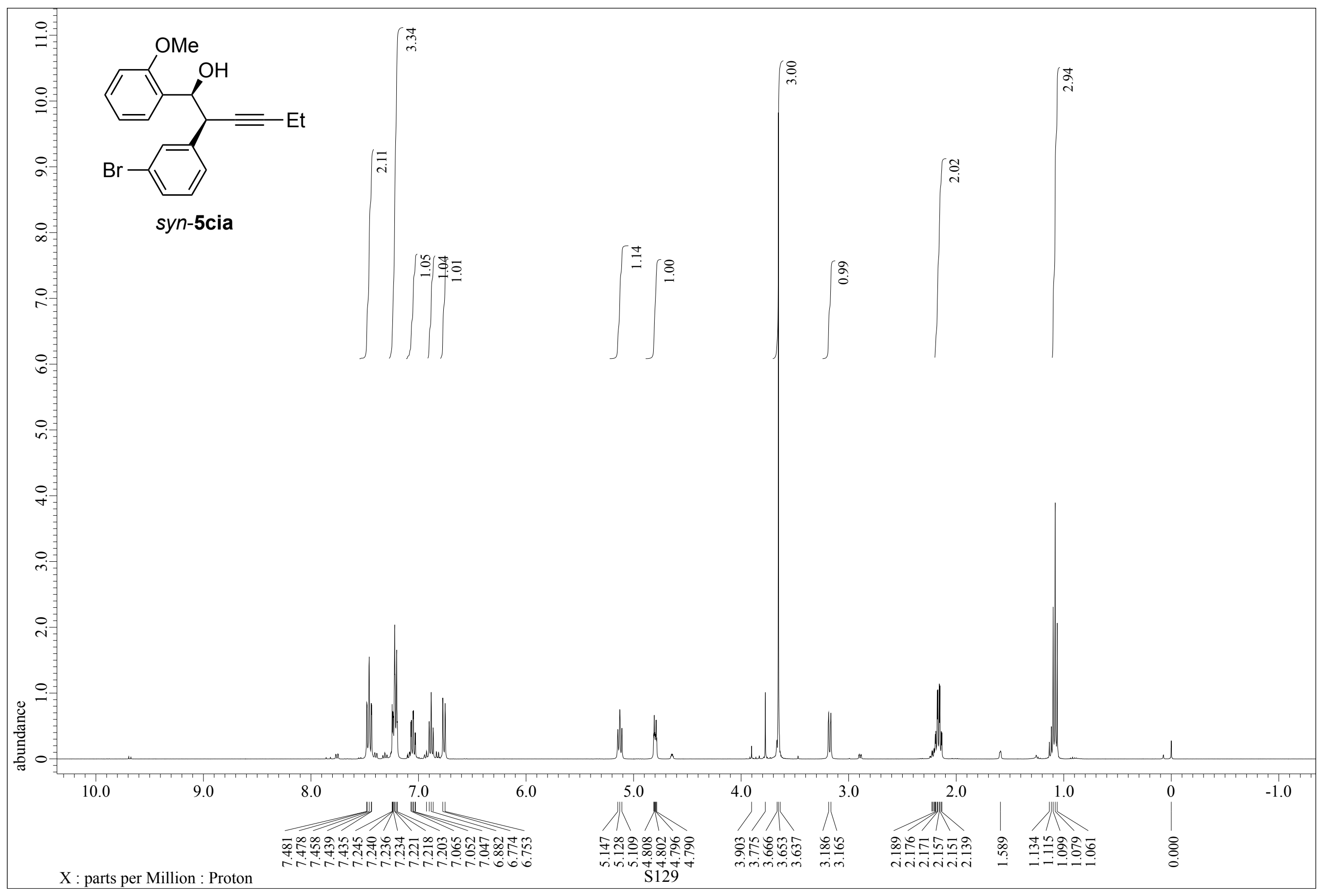




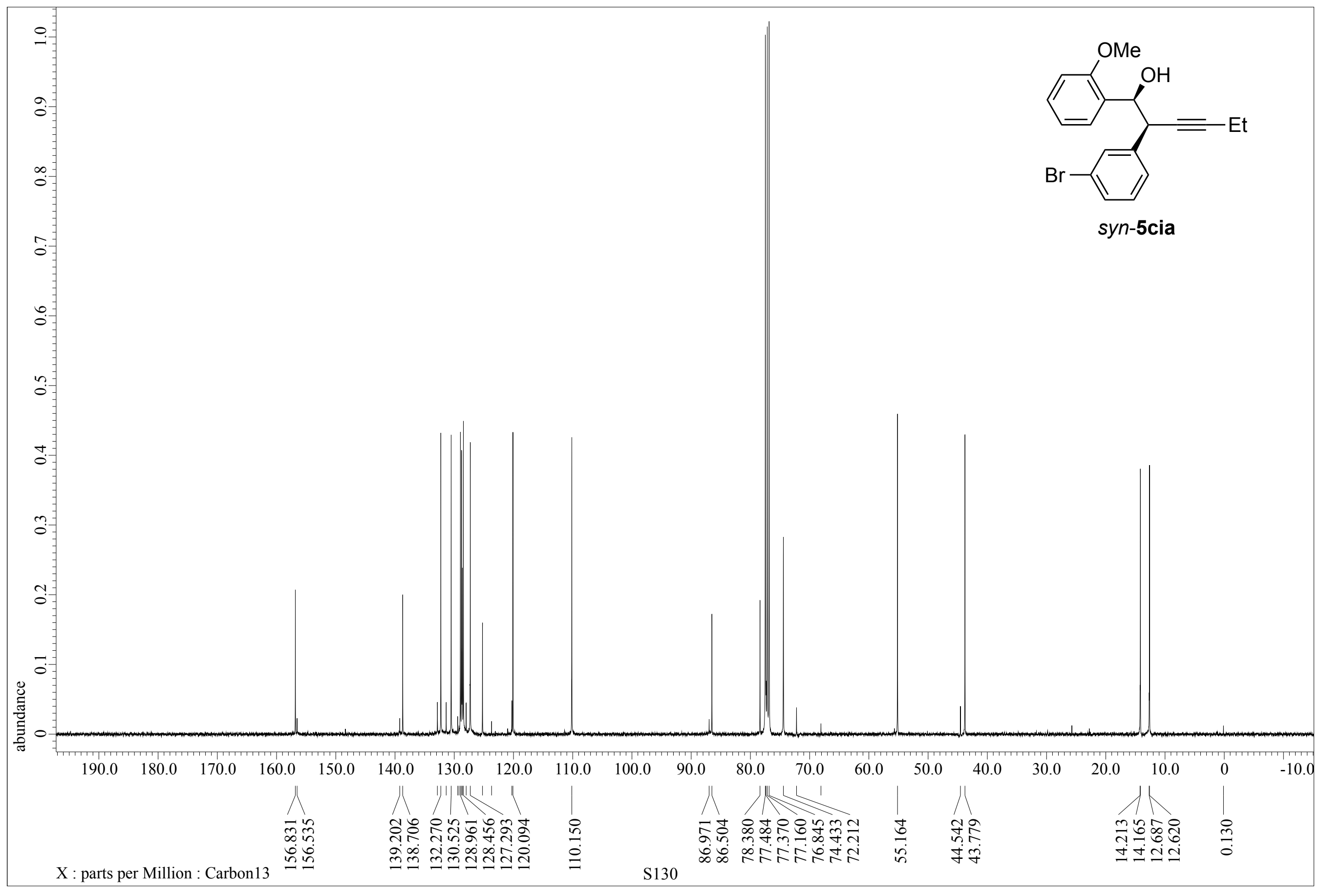




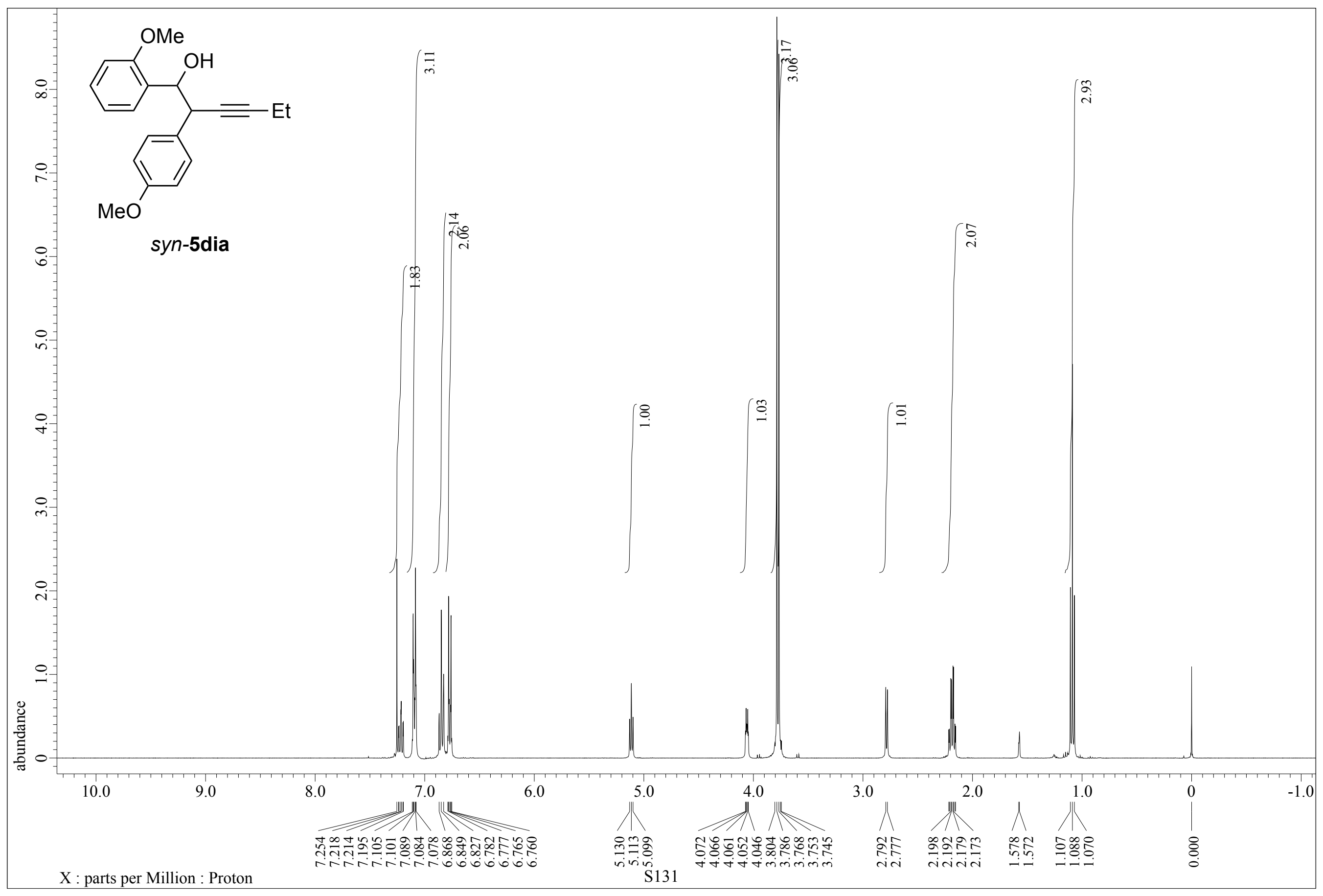




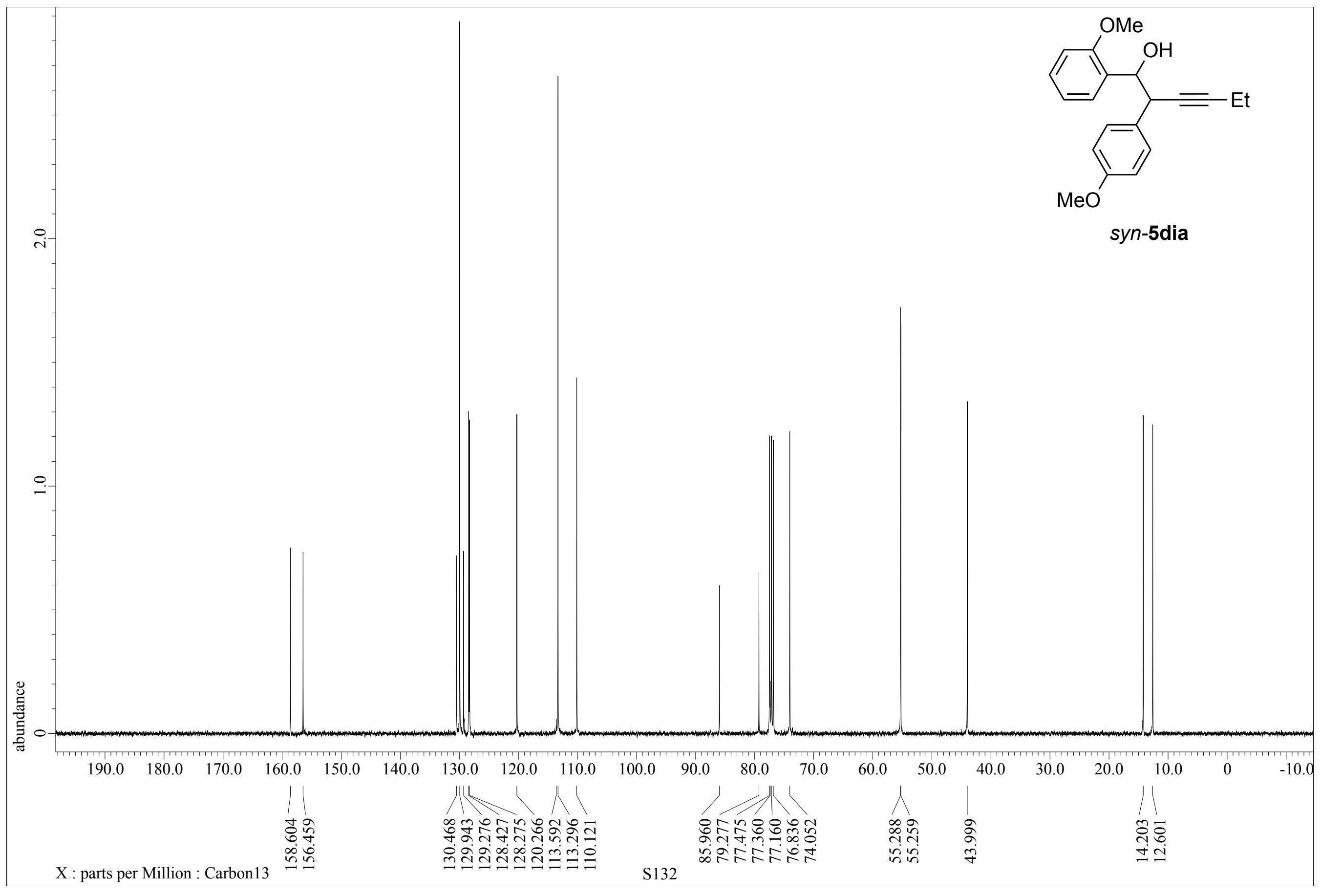




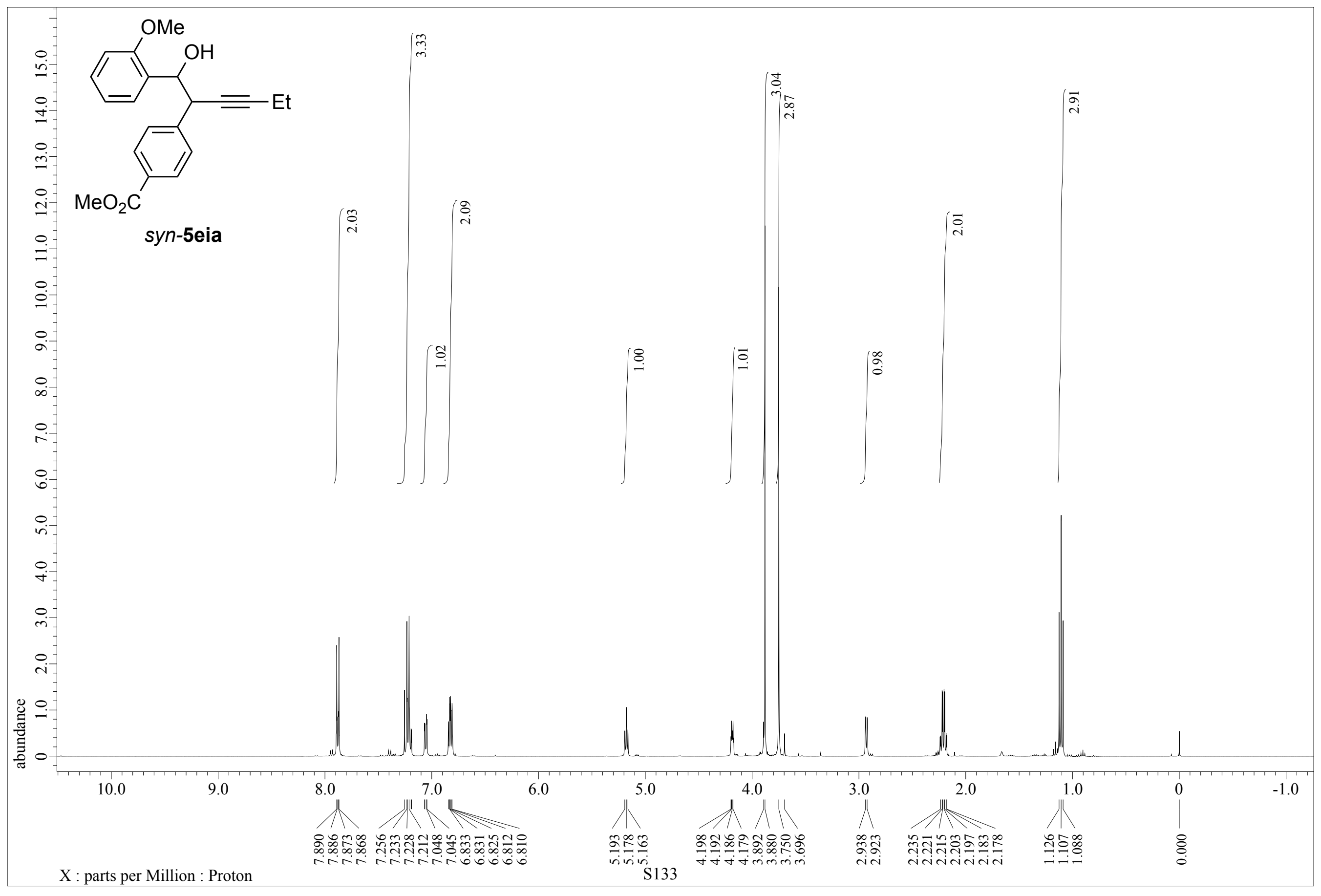




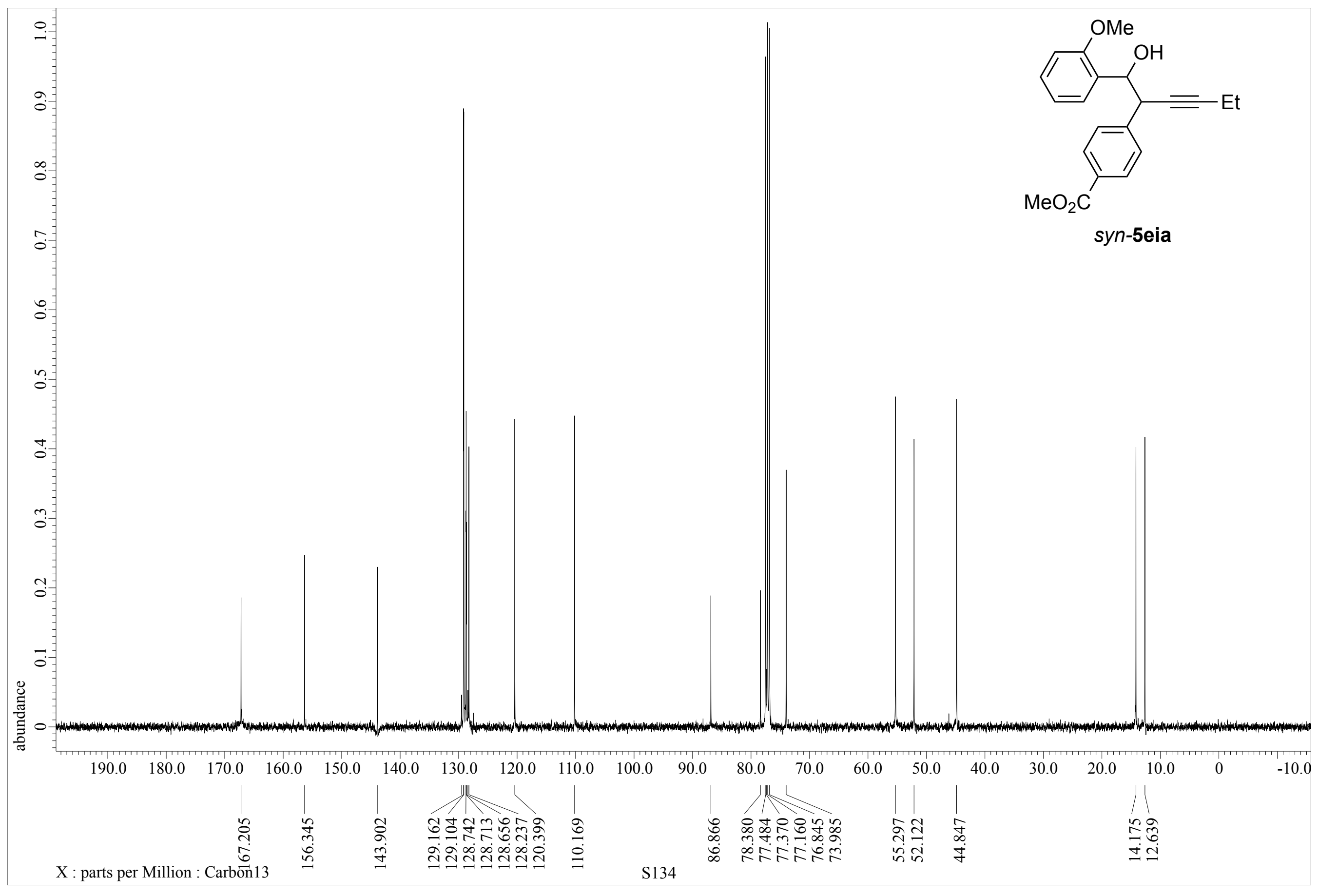




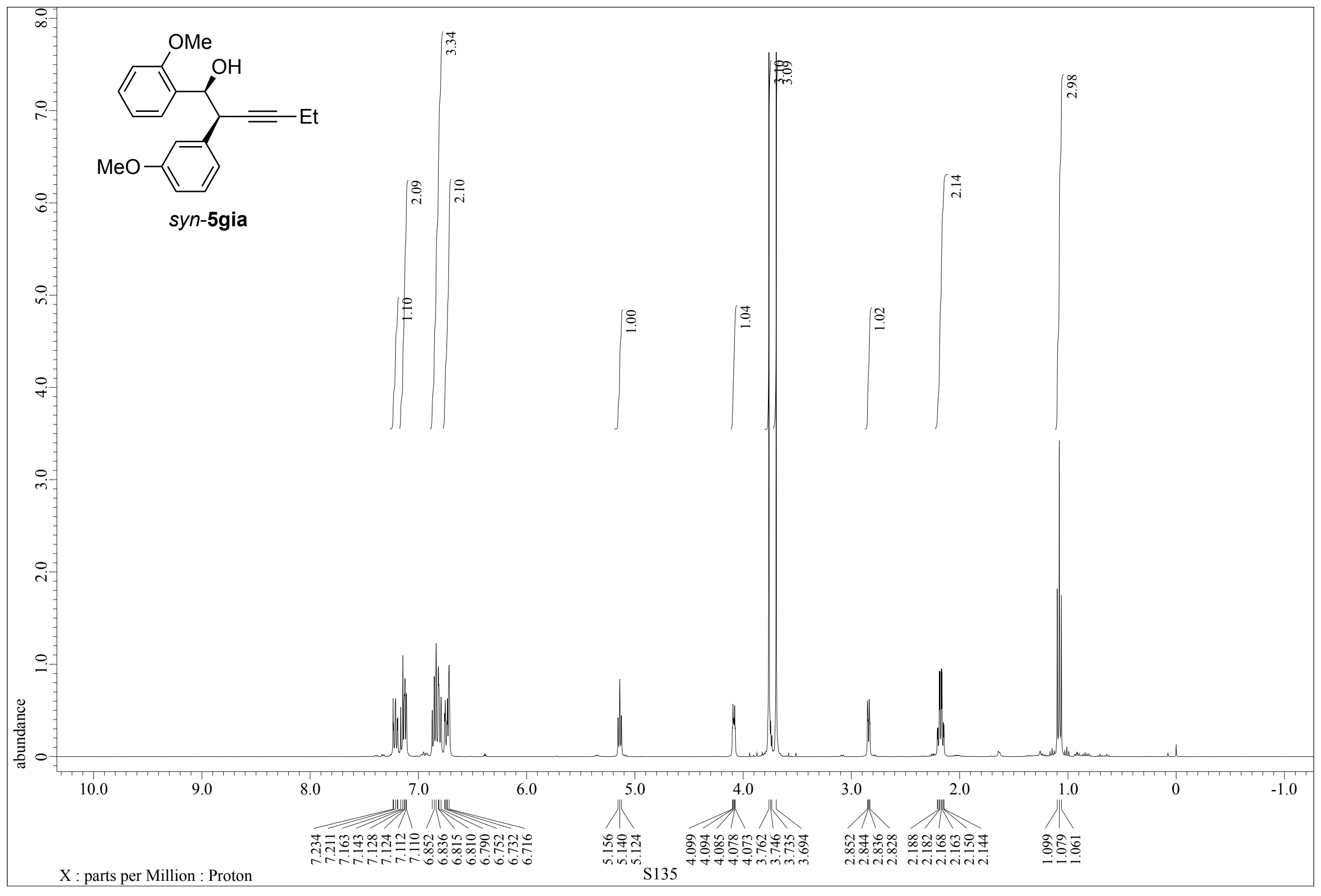




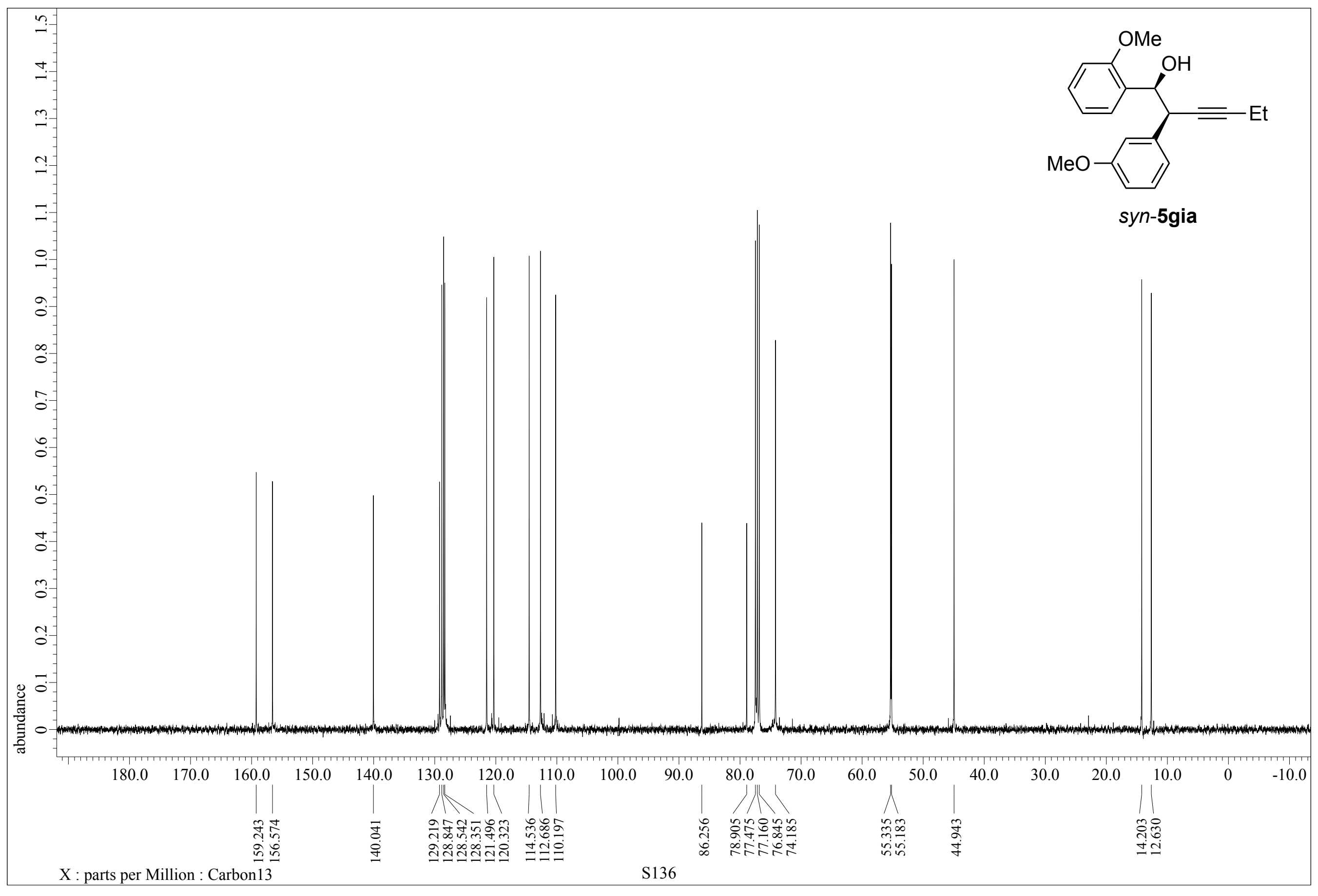




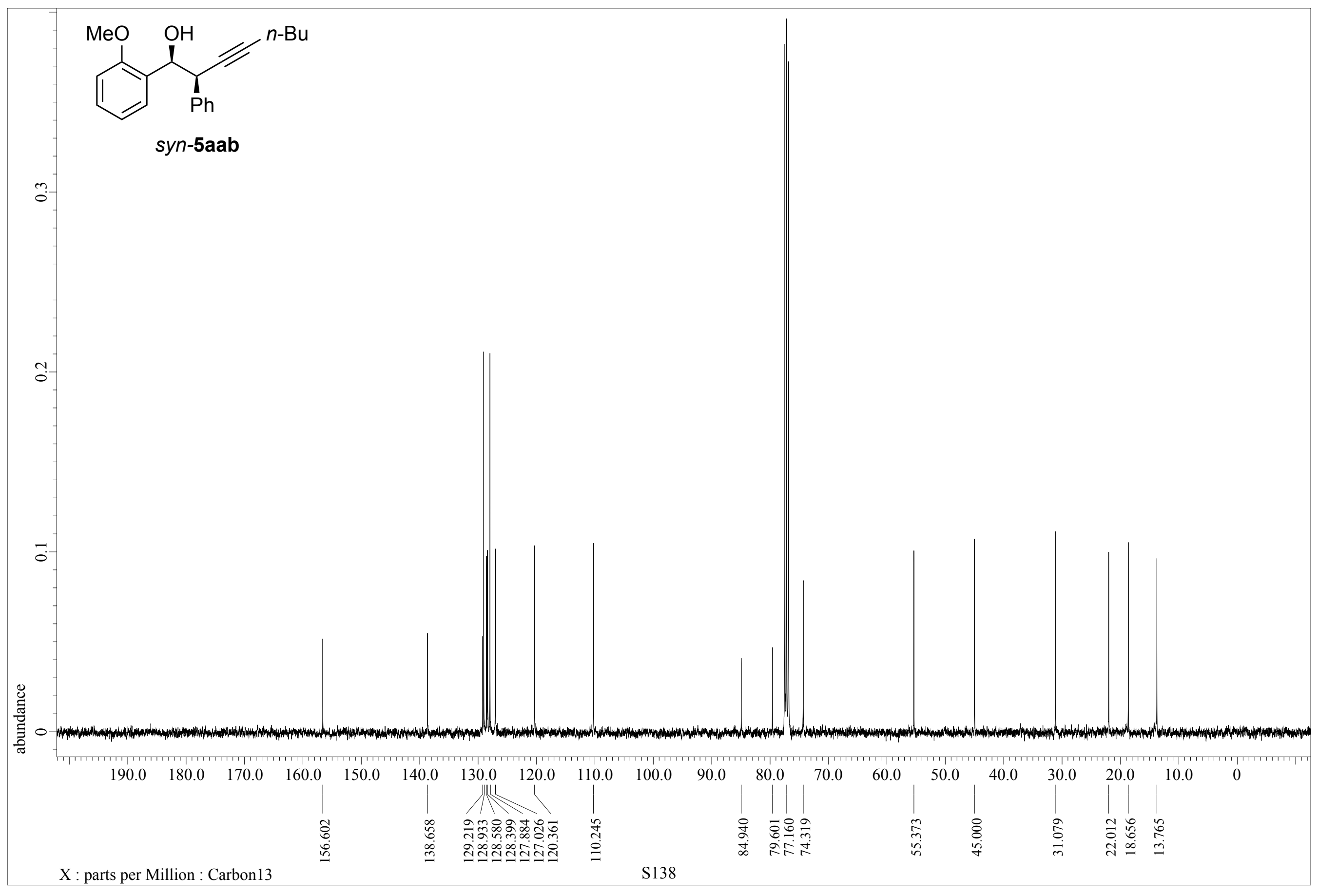




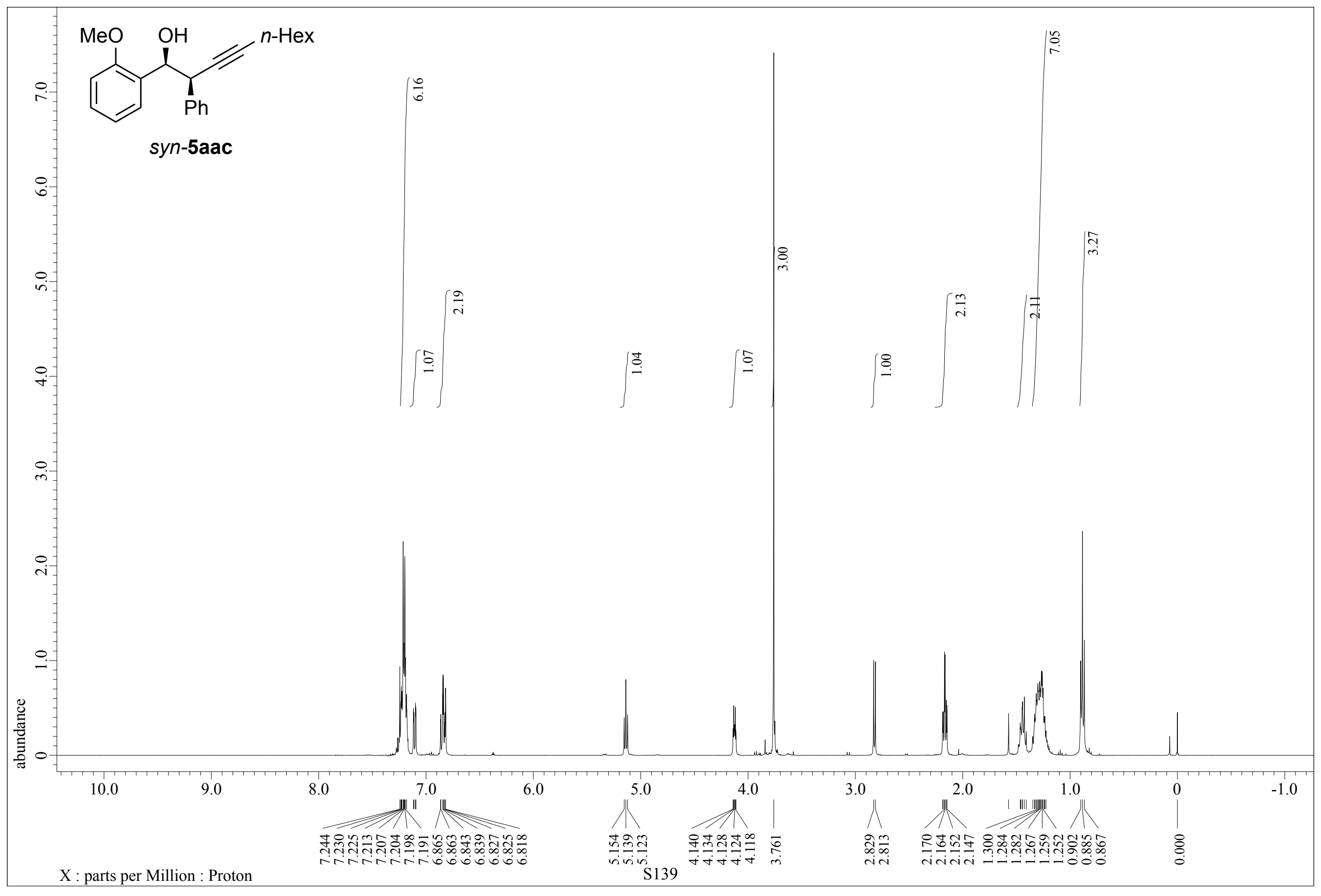




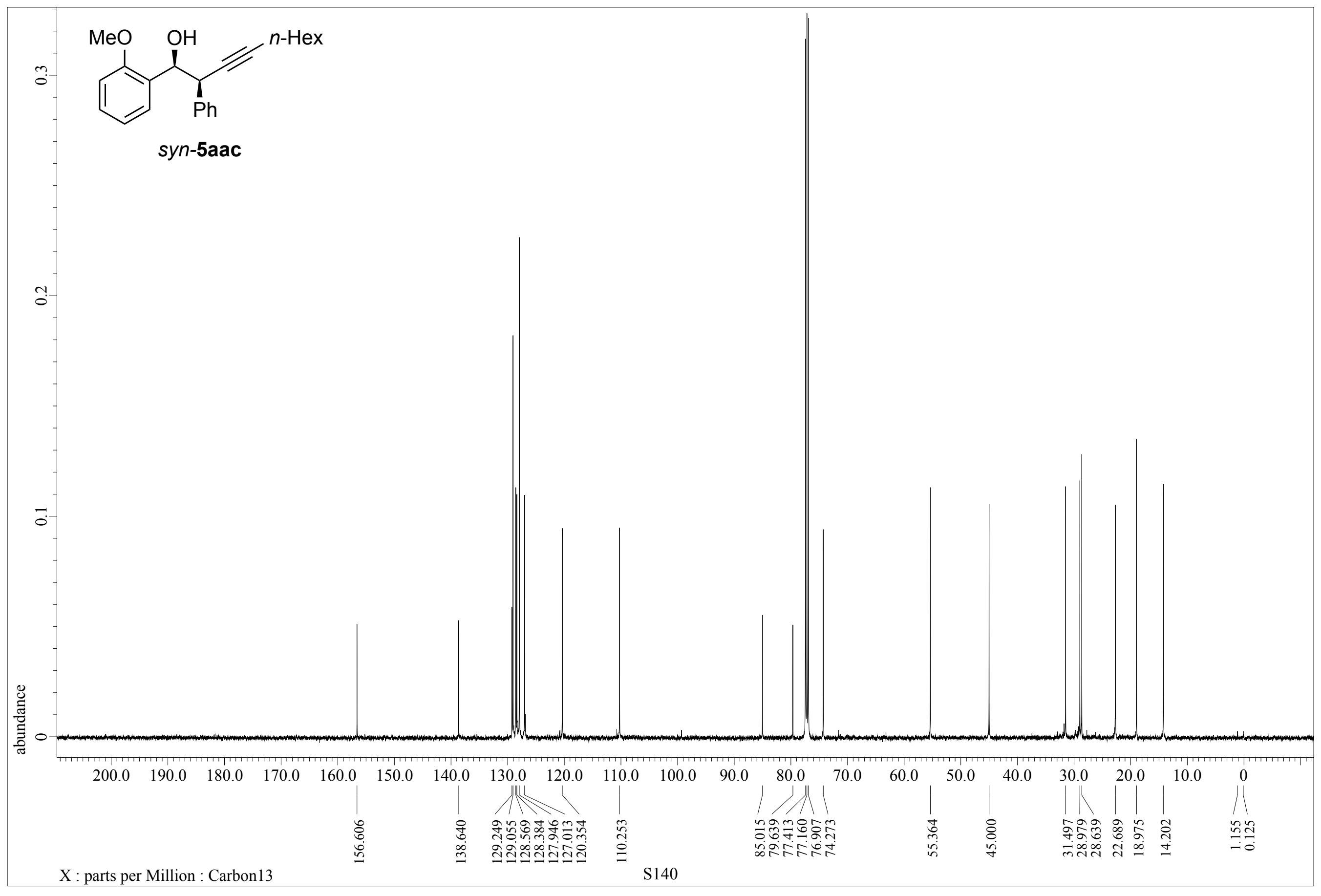




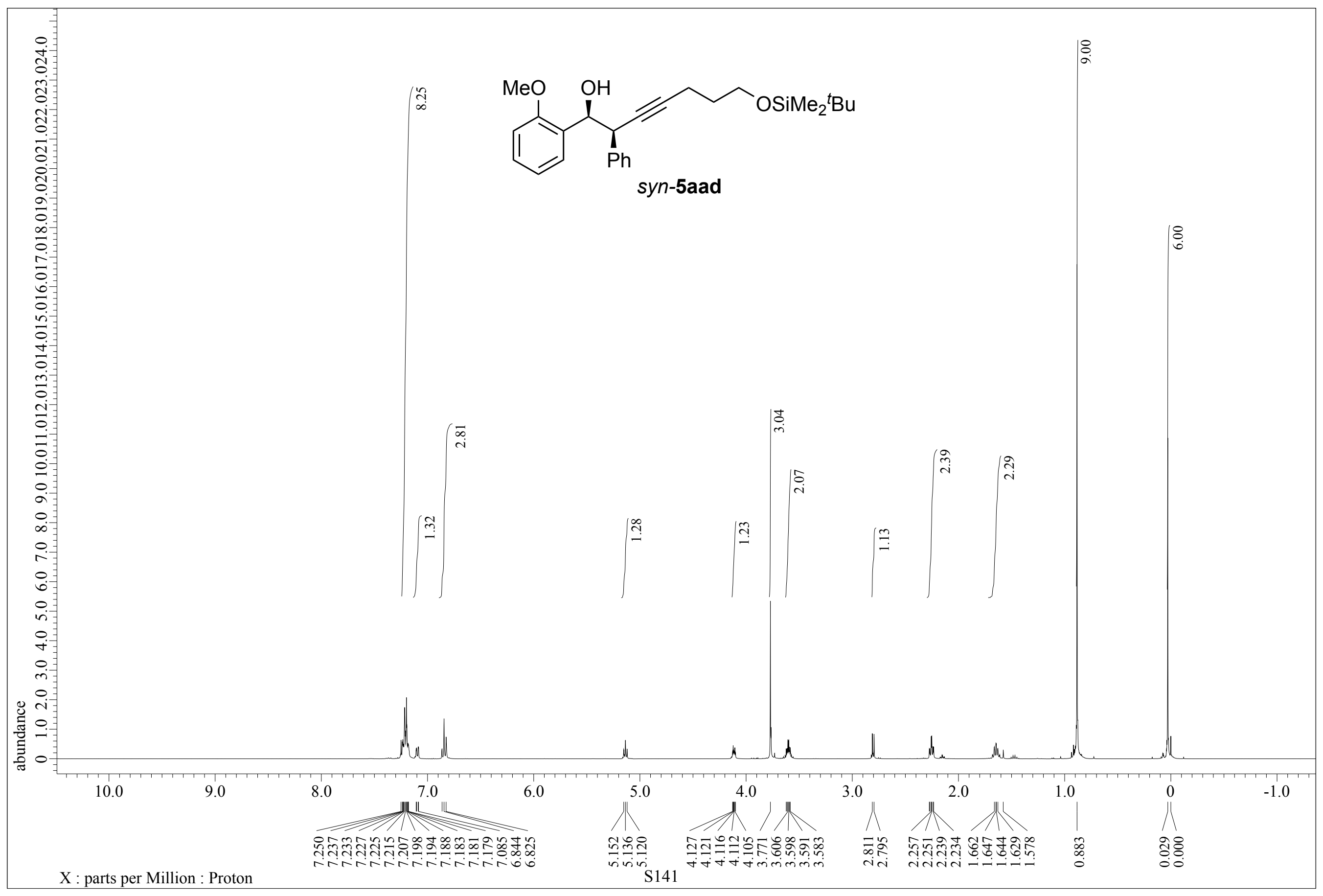




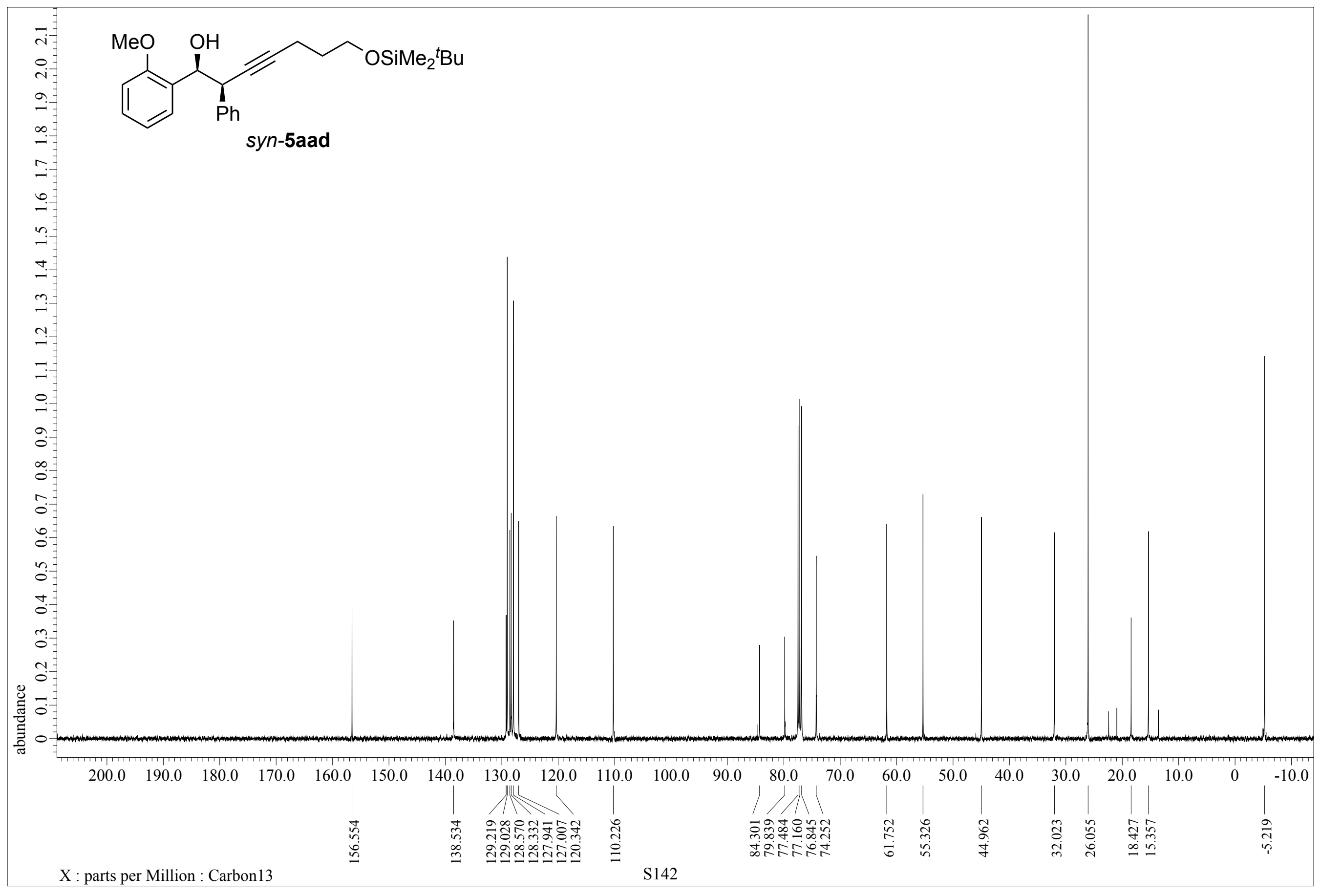




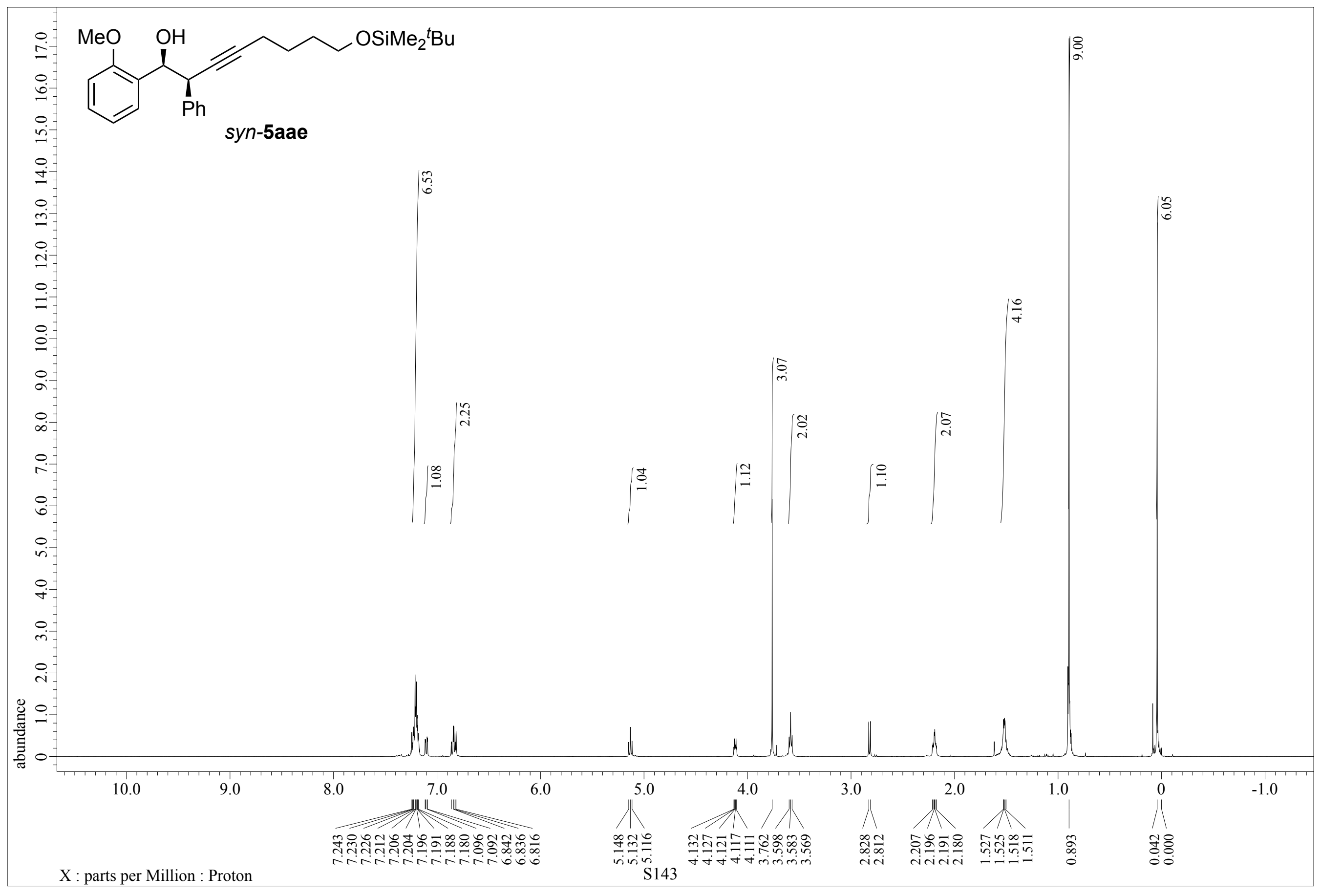




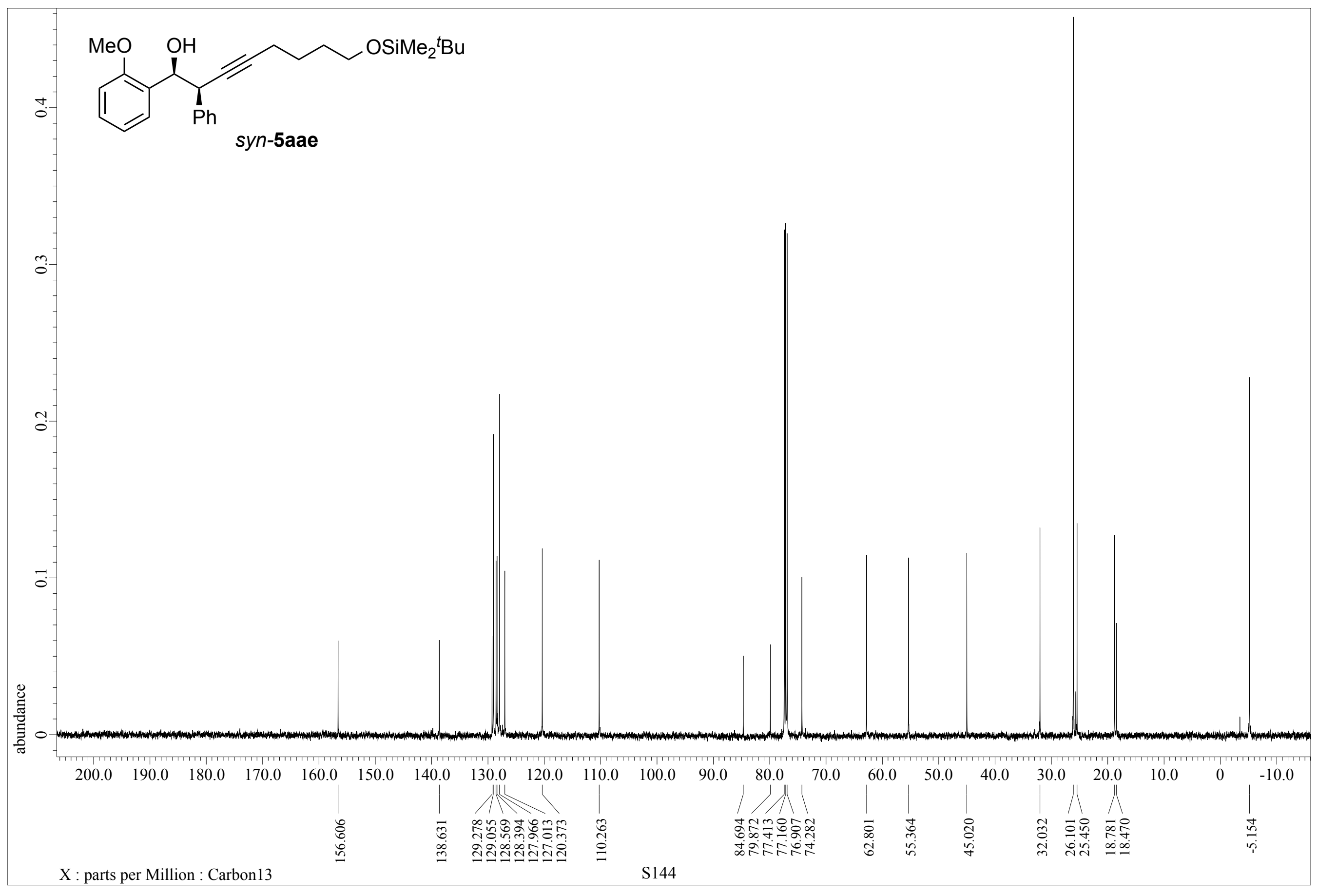




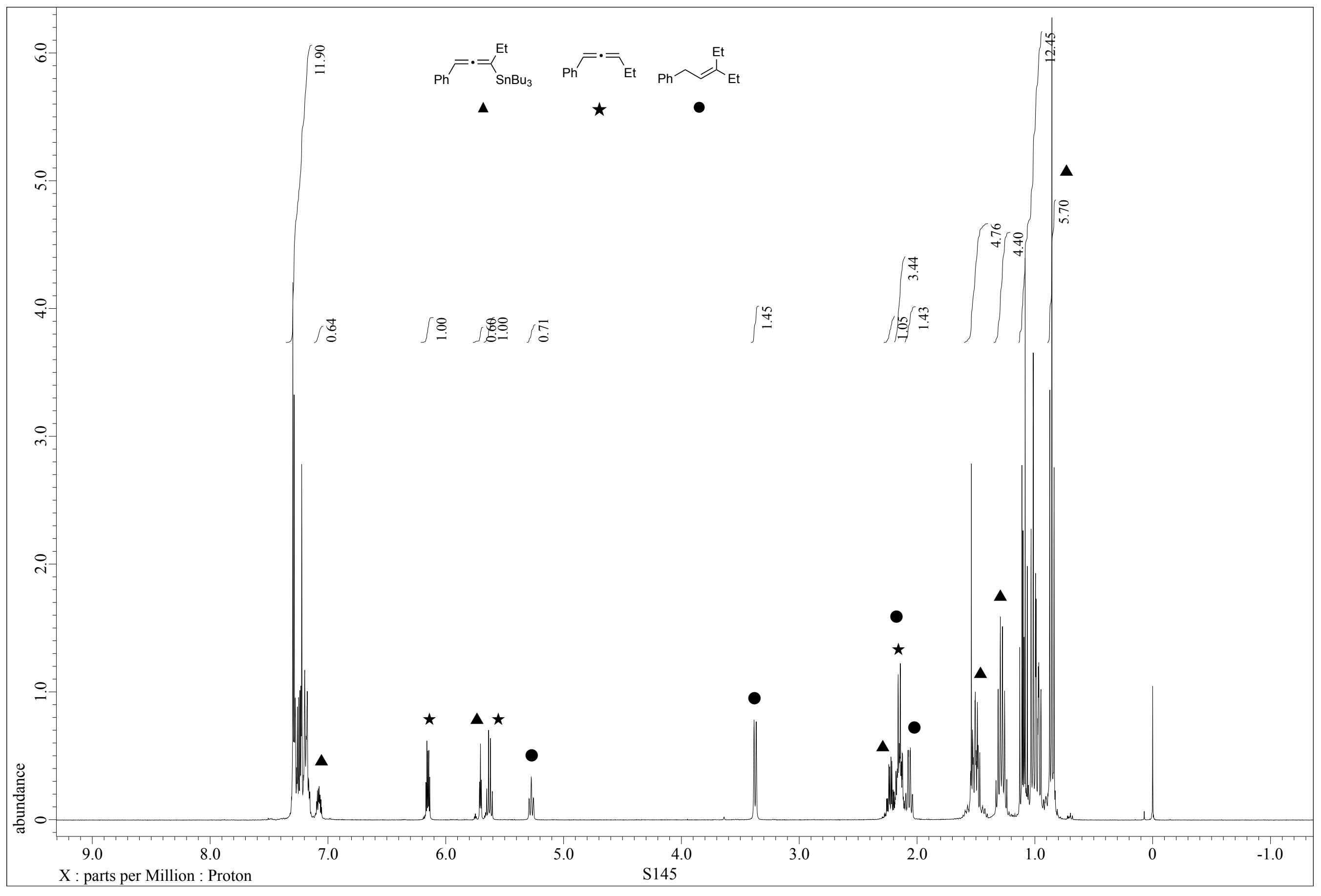




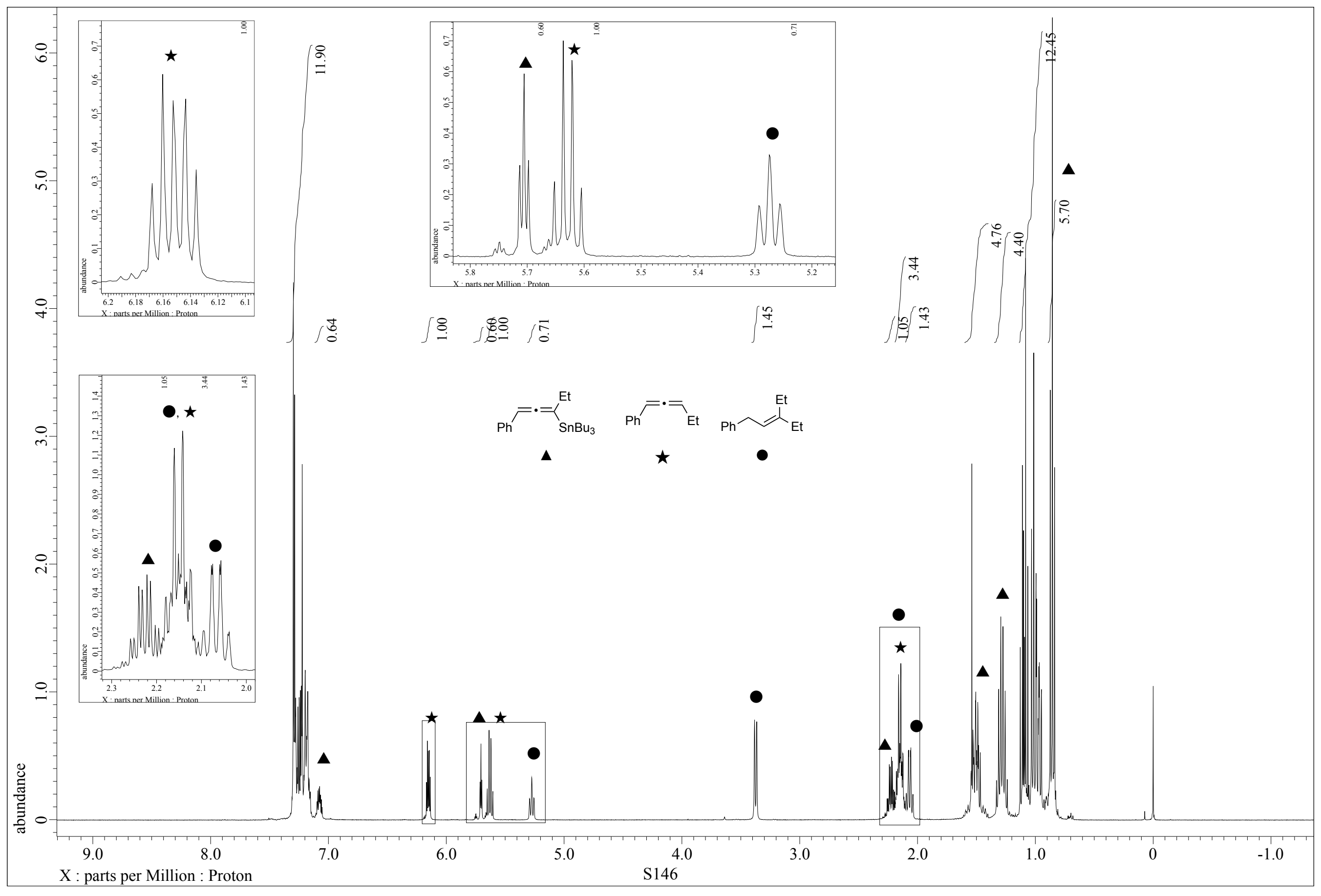




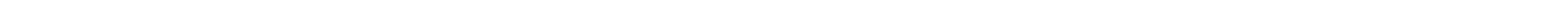




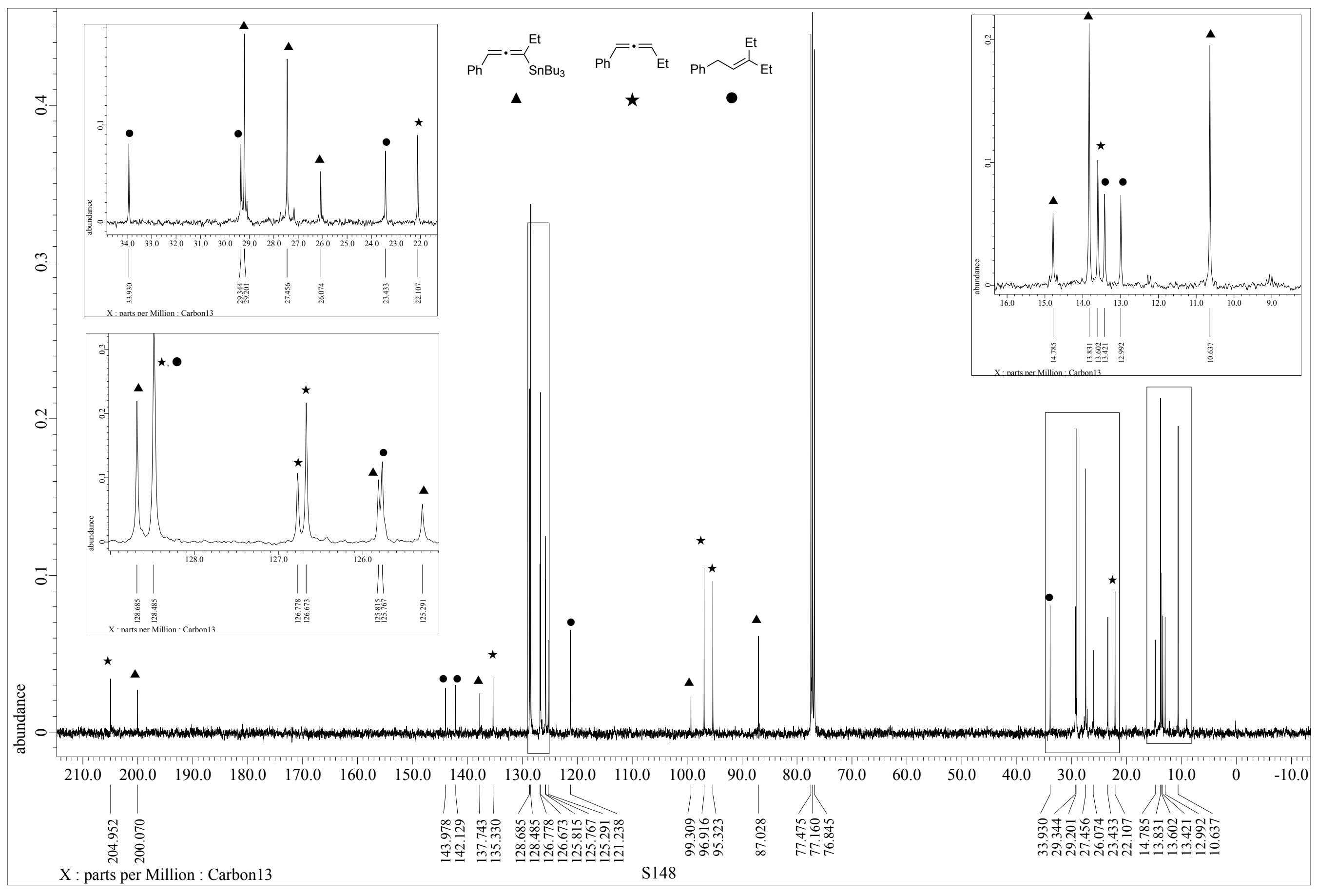




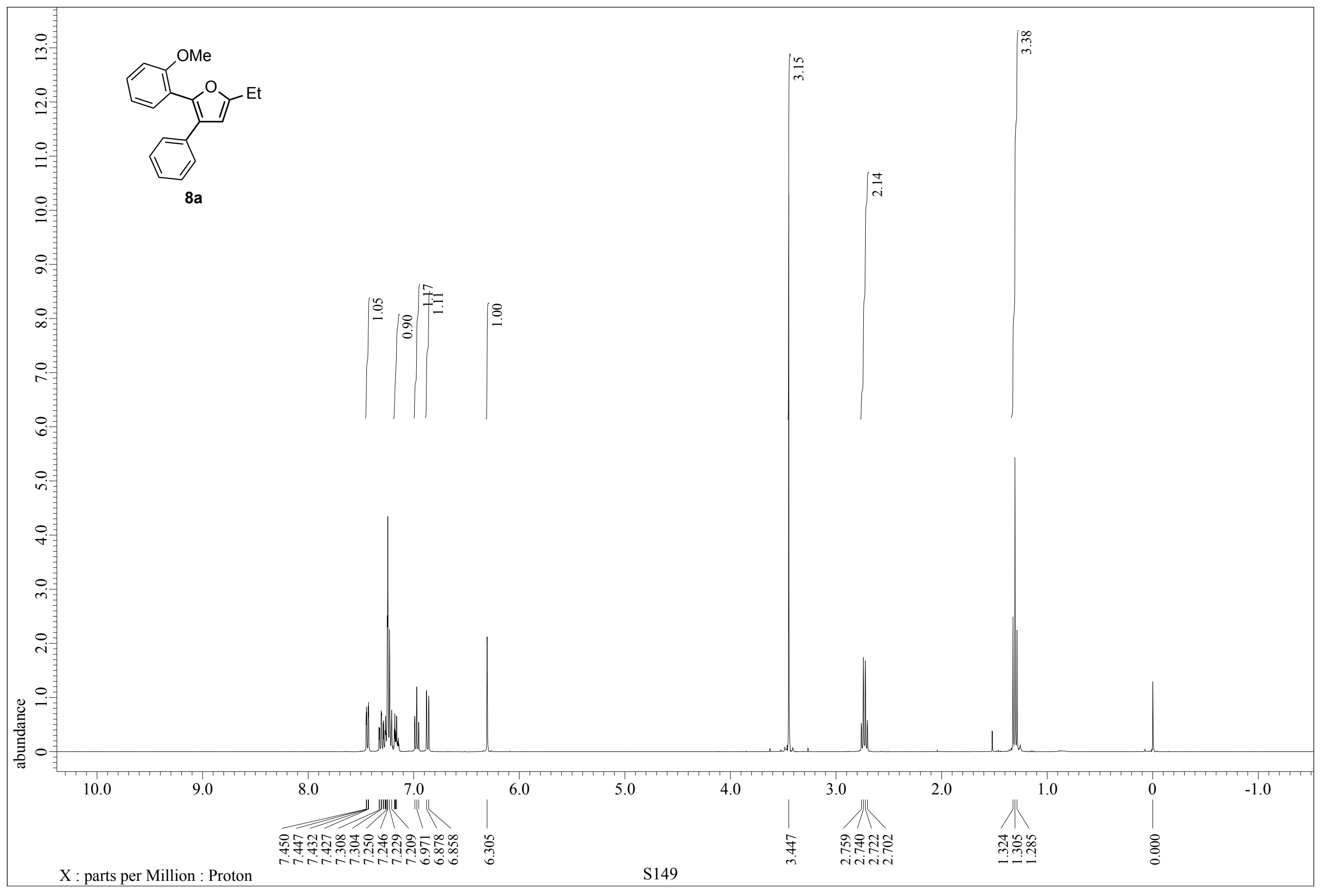




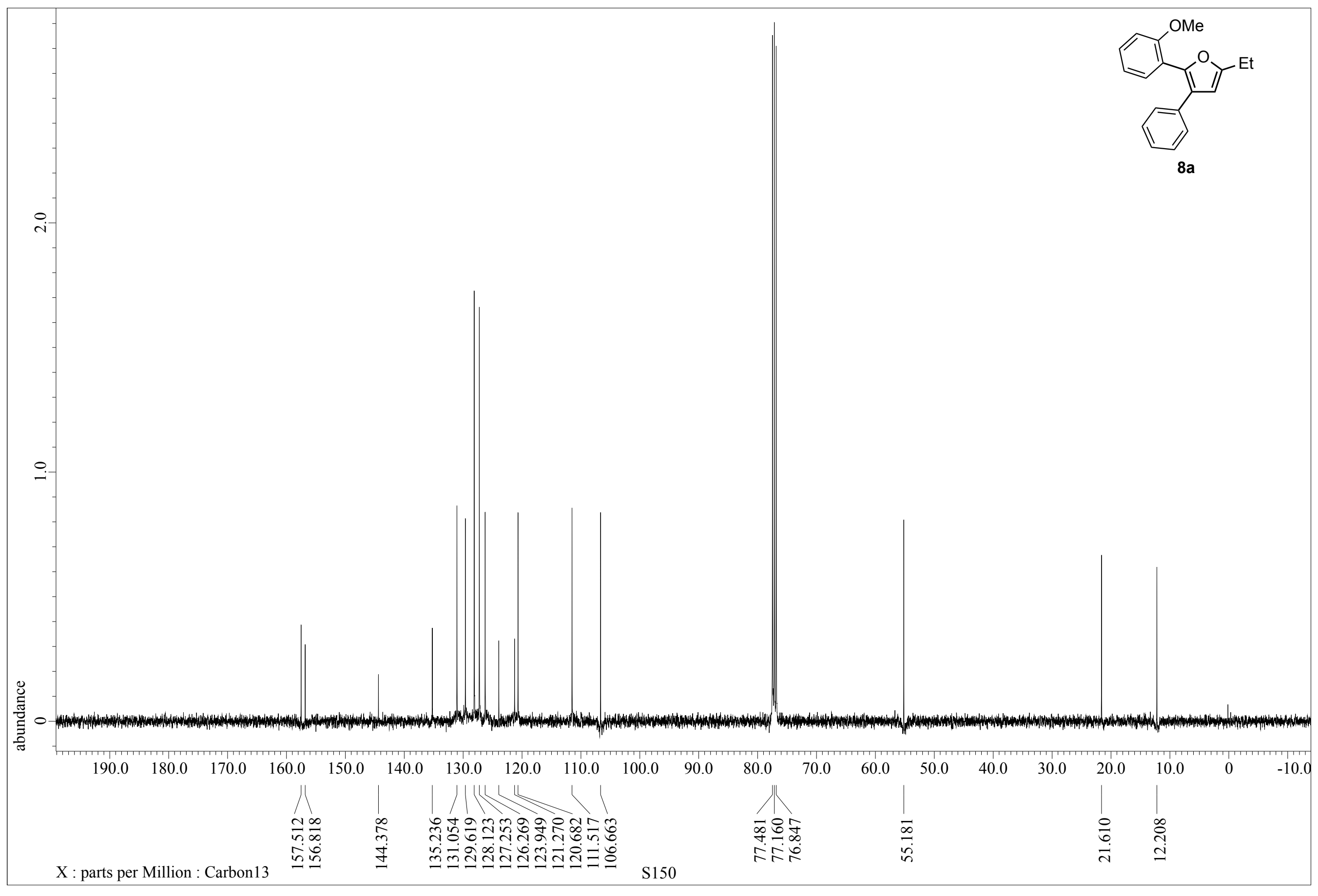




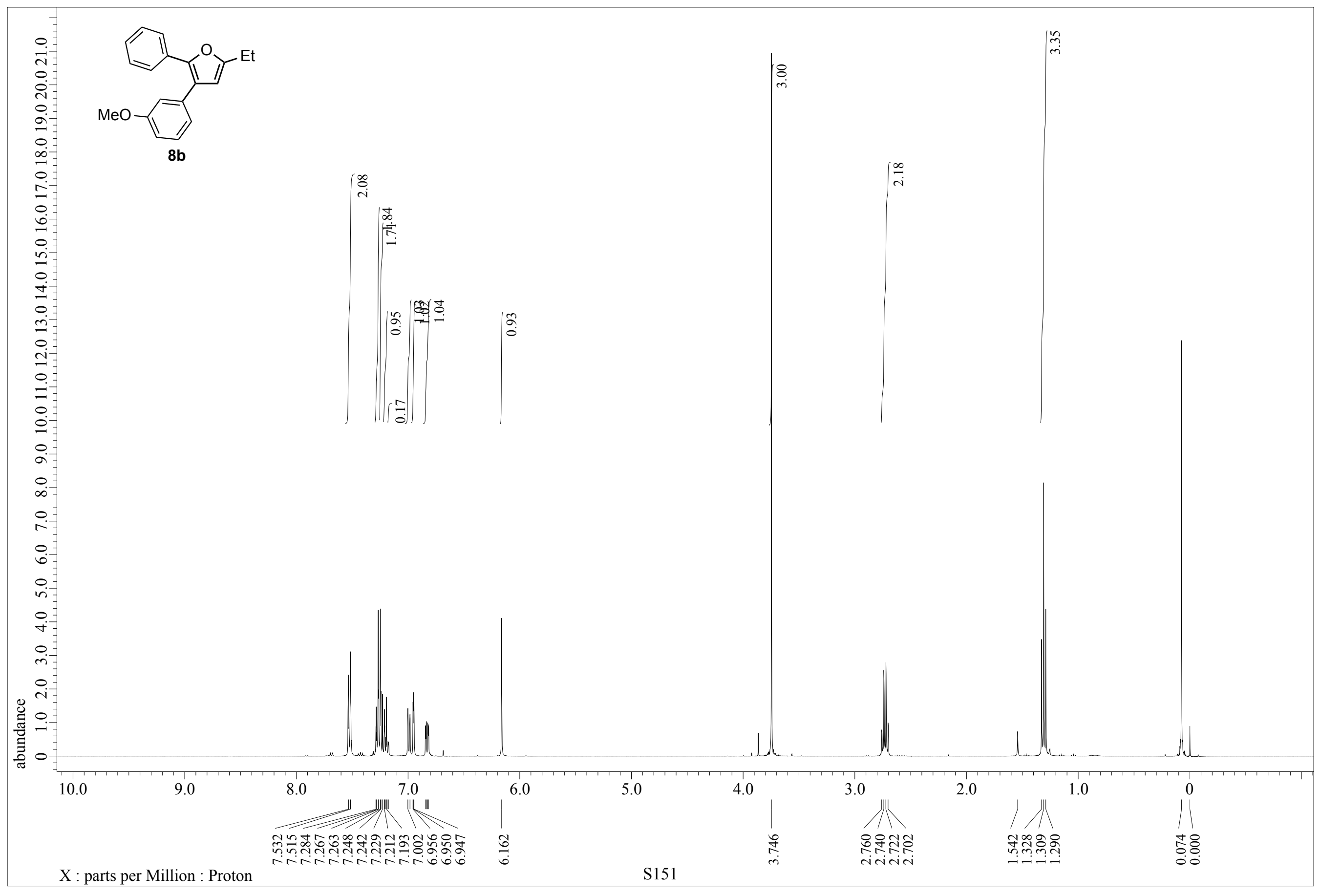




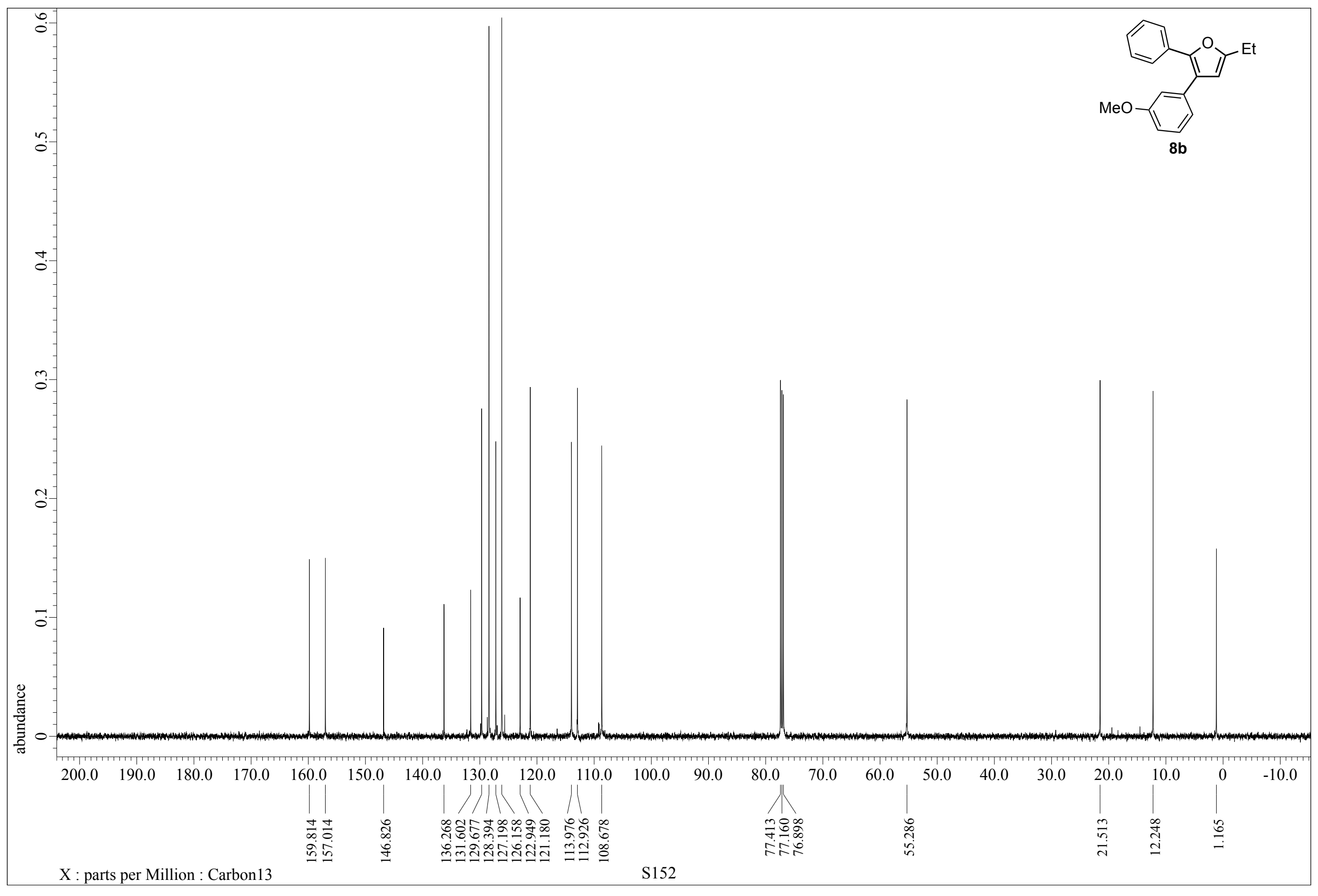

